\title{
Quality of life in inoperable non-small cell lung cancer
}

Citation for published version (APA):

Langendijk, J. A. (2000). Quality of life in inoperable non-small cell lung cancer. [Doctoral Thesis, Maastricht University]. Shaker Publishing BV. https://doi.org/10.26481/dis.20001215jl

Document status and date:

Published: 01/01/2000

DOI:

10.26481/dis.20001215jl

Document Version:

Publisher's PDF, also known as Version of record

\section{Please check the document version of this publication:}

- A submitted manuscript is the version of the article upon submission and before peer-review. There can be important differences between the submitted version and the official published version of record.

People interested in the research are advised to contact the author for the final version of the publication, or visit the DOI to the publisher's website.

- The final author version and the galley proof are versions of the publication after peer review.

- The final published version features the final layout of the paper including the volume, issue and page numbers.

Link to publication

\footnotetext{
General rights rights.

- You may freely distribute the URL identifying the publication in the public portal. please follow below link for the End User Agreement:

www.umlib.nl/taverne-license

Take down policy

If you believe that this document breaches copyright please contact us at:

repository@maastrichtuniversity.nl

providing details and we will investigate your claim.
}

Copyright and moral rights for the publications made accessible in the public portal are retained by the authors and/or other copyright owners and it is a condition of accessing publications that users recognise and abide by the legal requirements associated with these

- Users may download and print one copy of any publication from the public portal for the purpose of private study or research.

- You may not further distribute the material or use it for any profit-making activity or commercial gain

If the publication is distributed under the terms of Article $25 \mathrm{fa}$ of the Dutch Copyright Act, indicated by the "Taverne" license above, 
QUALITY OF LIFE IN INOPERABLE NON-SMALL CELL LUNG CANCER 



\section{QUALITY OF LIFE IN INOPERABLE NON-SMALL CELL LUNG CANCER}

\section{PROEFSCHRIFT}

ter verkrijging van de graad van doctor aan de Universiteit Maastricht op gezag van de Rector Magnificus,

Prof. Dr. A.C. Nieuwenhuijzen Kruseman, volgens besluit van het College van Dekanen, in het openbaar te verdedigen op vrijdag, 15 december 2000 , 0m 12.00 uur

Door

Johannes Albertus Langendijk

Geboren te Amsterdam in 1963 


\section{Promotor:}

Prof. Dr. E.F.M. Wouters

\section{Co-promotores:}

Dr. G.P.M. ten Velde

Dr. J.M.A. de Jong (Radiotherapeutisch Instituut Limburg)

\section{Beoordelingscommissie:}

Prof. Dr. H.F.P. Hillen (voorzitter)

Prof. Dr. J.C. de Haes (Universiteit van Amsterdam)

Prof. Dr. P. Lambin

Prof. Dr. J.J. Manni

Prof. Dr. E.M. Noordijk (Universiteit Leiden)

C) Copyright Shaker Publishing 2000

All rights reserved. No part of this publication may be reproduced, stored in a retrieval system, or transmitted, in any form or by means, electronic, mechanical, photocopying, recording or otherwise, without permission of the publishers.

Printed in the Netherlands.

ISBN 90-423-0122-8

Shaker Publishing BV

St. Maartenslaan 26

6221 AX Maastricht

Tel.: 043: 3500424

Fax: 043-3255090

Financial support for the publication of this thesis was given by Glaxo Welcome, Nucletron, Varian, Schering-Plough, Janssen Cilag, Astra Zenaca and the R.T.I.L.. Their help is gratefully acknowledged. 


\section{Contents}

$\begin{array}{lll}\text { Chapter } 1 \quad \text { General introduction } & 9\end{array}$

$\begin{array}{lll}\text { Chapter } 2 & \text { Aims of this thesis } & 29\end{array}$

Chapter 3 Pre-treatment quality of life of inoperable non-small cell lung 35 carcinoma patients referred for primary radiotherapy.

Chapter 4 The prognostic impact of quality of life assessed with the EORTC

QLQ-C30 in inoperable non-small cell lung carcinoma treated with radiotherapy.

Chapter 5 Is the chest radiograph a reliable tool in the assessment of tumor response after radiotherapy in non-small cell lung carcinoma?

Chapter $6 \quad$ Quality of life after palliative radiotherapy in non-small cell lung cancer: a prospective study.

Chapter $7 \quad$ A prospective study on quality of life before and after radical radiotherapy in non-small cell lung cancer.

Chapter 8 Quality of life after curative radiotherapy in stage I non-small cell lung cancer.

Chapter 9 Massive haemoptysis after radiotherapy in inoperable non-small cell lung cancer: is endobronchial brachytherapy really a risk factor?

Chapter 10 External irradiation versus external irradiation plus endobronchial brachytherapy in inoperable non-small cell lung cancer: A prospective randomised study.

Chapter 11 General discussion

Summary

Samenvatting

Dankwoord

Curriculum vitae 

Chapter 1

General introduction 


\section{口 Introduction}

Lung cancer is the leading cause of cancer-related death '. In the Netherlands, approximately 9000 new cases are diagnosed each year and more than 8000 patients will die from this malignant disease ${ }^{1}$. According to the classification system of the World Health Organization ${ }^{2}$, lung cancer can be grouped into five main tumour categories, including squamous cell carcinoma, adenocarcinoma, large cell carcinoma, adenosquamous cell carcinoma and small cell lung carcinoma (SCLC). The first four histological types are often referred to as non-small cell lung carcinoma (NSCLC). In early stage NSCLC, surgery is considered the most effective treatment modality ${ }^{3}$. However, approximately $75 \%$ of the cases are not eligible for a curative resection, mainly because of advanced loco-regional tumour extension, the presence of distant metastases and/or comorbid diseases. These inoperable cases are often referred for radiotherapy. Prognosis in inoperable NSCLC depends on a number of prognostic factors. In inoperable NSCLC, performance status ${ }^{4-12}$, weight loss ${ }^{5,7,11-14}$ and stage of disease ${ }^{6,8-11}$ are the most important and well-accepted prognostic factors. In general, the outcome for most patients with inoperable tumours remains poor. The overall five year survival rate for stage I and II ${ }^{15}$ treated with curative radiotherapy varies from $6 \%$ to $27 \%{ }^{16-27}$. For stage IIIa and IIIb treated with primary radiotherapy to a total dose of at least $60 \mathrm{~Gy}$, median survival rates are achieved ranging from 8 to 14 months ${ }^{28-30}$. In this group of patients, the five-year survival rate is less than $5 \%$. Traditionally, treatment outcome in lung cancer is expressed in terms of response to treatment, loco-regional tumour control, disease free survival and overall survival. Although recent meta-analysis has shown that the addition of chemotherapy to radiotherapy can have a statistically significant survival benefit compared to radiotherapy alone ${ }^{31}$, the differences between these two modalities as measured with the traditional outcome parameters remain small. Taking into account the relatively poor prognosis in inoperable NSCLC and the minimal gain achieved with new modalities regarding the traditional endpoints, it becomes increasingly important to incorporate quality of life (QoL) as an endpoint into clinical studies. The importance of evaluating QoL parameters into the treatment of patients with lung cancer becomes generally accepted ${ }^{32-}$ ${ }^{34}$. However, the implementation of evaluating QoL in clinical practice has been limited. 
Consequently, detailed information concerning QoL specifically among patients with inoperable NSCLC treated with radiotherapy is scarce.

\section{Defining quality of life}

Difficulties arise regarding what is meant exactly by the term 'quality of life'. Generally, QoL is used synonymously for subjective or general well-being ${ }^{35}$ and may be affected by dimensions such as social network, financial status, political environment and prevalence of disease. Although these specific topics may influence QoL, they are less relevant in the assessment of QoL in the oncologic practice. In patients with malignant diseases, the attention is usually focused on QoL dimensions related to the disease, its treatment, or the side-effects of treatment, also referred as to health-related quality of life. There is now general agreement that quality of life in the context of health is a multidimensional concept concerned with the impact of physical symptoms and side effects of treatment on patients' functioning and psychosocial well being ${ }^{36}$. Major health-related dimensions include physical (symptoms of disease, side effects of treatment, symptomatic distress), functional (activity level, cognitive status, role status, sexuality), psychological (emotional well-being, emotional distress), social (relationships, work role, leisure, financial) and spiritual domains (meaning of life, religious issues) ${ }^{37}$.

Secondly, QoL should be regarded as a subjective experience and therefore the emphasis should be placed on assessing the subjective experience of the person whose quality of life is in question ${ }^{36}$. In this thesis, the term QoL is used as a synonym for health-related QoL.

\section{Quality of life assessment}

\section{Performance status}

Traditionally, performance status has been widely used as a measure for QoL. Karnofsky was one of the first who introduced an instrument (The Karnofsky Performance Status Scale (KPS)) to measure QoL systematically (Table 1) ${ }^{38-39}$. The Eastern Cooperative 
Oncology Group (ECOG) performance status scale (Table 2) and the World Health Organization $(\mathrm{WHO})$ performance status scale are comparable tools ${ }^{40}$. Unfortunately, these rather simple methods have a number of limitations. First, by using performance status to evaluate QoL, only a limited range of dimensions can be measured. For

Table 1: Karnofsky performance status

\begin{tabular}{|c|c|c|}
\hline Definition & Score & Criteria \\
\hline \multirow{3}{*}{$\begin{array}{l}\text { Able to carry out on normal cativity and to work. } \\
\text { No sprecial care is needed }\end{array}$} & 100 & Normal; no complaints; no evidence of disease \\
\hline & 90 & $\begin{array}{l}\text { Able to carry out normal activity; minor signs or } \\
\text { symptoms of disease }\end{array}$ \\
\hline & 80 & $\begin{array}{l}\text { Normal activity with effort; some signs or symptoms } \\
\text { of disease }\end{array}$ \\
\hline \multirow{3}{*}{$\begin{array}{l}\text { Unable to work. Able to live at home, care for } \\
\text { most personal needs. Cares for self. Unable to } \\
\text { carry on normal activity or to do active work. }\end{array}$} & 70 & A varying amount of assistance is needed \\
\hline & 60 & $\begin{array}{l}\text { Requires occasional assistance, but is able to care } \\
\text { for most of his/her needs }\end{array}$ \\
\hline & 50 & $\begin{array}{l}\text { Requires considerable assistance and frequent } \\
\text { medical care }\end{array}$ \\
\hline \multirow{5}{*}{$\begin{array}{l}\text { Unable to care for self. Requires equivalent of } \\
\text { institutional or hospital care. Disease may be } \\
\text { progressing rapidly. }\end{array}$} & 40 & Disabled; requires special care and assistance \\
\hline & 30 & $\begin{array}{l}\text { Severely disabled; hospitalization is indicated } \\
\text { although not imminent }\end{array}$ \\
\hline & 20 & $\begin{array}{l}\text { Very sick; hospitalization necessary; active } \\
\text { supportive treatment necessary }\end{array}$ \\
\hline & 10 & Moribund; fatal processes progressing rapidly \\
\hline & 0 & Dead \\
\hline
\end{tabular}

instance, the Karnofsky score evaluates only three dimensions of health, including the ability to work, the ability to carry out normal activity and the ability to self-care. Factors such as social status, sexual functioning, occupation, cognitive functioning and psychological status ${ }^{41-42}$ are not taken into account but certainly will affect patients' QoL. Furthermore, performance status reflects the impression of the physician of patients' QoL. It has been emphasised that the assessment of QoL is essentially subjective $36,43,44$. Assessment of QoL by health care professionals is not only inappropriate but also inaccurate. Performance status has not only been found unreliable with inconsistent 
ratings of the same patient by different observers ${ }^{45}$ but has also been found to be correlating poorly with patients' own report of QoL ${ }^{46-49}$.

In the last decade, much effort has been employed in designing instruments to assess health-related QoL in a more appropriate way. Most instruments are self-reported questionnaires containing a number of questions or items grouped within domains or dimensions of related attributes. An optimal QoL instrument should at least assess several of the aforementioned dimensions of health-related QoL.

Table 2: ECOG-performance status

\begin{tabular}{ll}
\hline Grade & Criteria \\
\hline 0 & Fully active, able to carry on all predisease activities without restriction \\
1 & $\begin{array}{l}\text { Restricted in physically strenuous activity but ambulatory and able to carry } \\
\text { ou work of a light or sedentary nature. For example, light housework, office } \\
\text { work. }\end{array}$ \\
2 & $\begin{array}{l}\text { Ambulatory and capable of self-care but unable to carry out any work } \\
\text { activities. Up and about more than } 50 \% \text { of waking hours. }\end{array}$ \\
Capable of only limited self-care, confined to bed or chair $50 \%$ or more of \\
waking hours.
\end{tabular}

\section{Psychometric properties}

To be used in clinical practice, QoL instruments have to be reliable and valid. A number of methods have been employed to investigate the validity of QoL instruments. An instrument is considered valid when it measures what is intended to measure. The following aspects of validity can be tested:

Convergent validity. Convergent validity refers to the correlation among data for factors that measure items of the same scale (corrected for overlap) ${ }^{50}$. Convergent validity can thus only be tested for multi-item scales. 
Divergent validity. Divergent validity refers to the ability to distinguish items, designed to assess different aspects of $\mathrm{QoL}{ }^{51}$. For instance, cognitive functioning and nausea \& vomiting have less in common and are expected to be poorly correlated.

Construct validity. Construct validity refers to the ability of an instrument to measure what is important to patients ${ }^{50}$. It can be examined by testing the correlation among scales that are theoretically related, such as role and physical functioning.

Predictive validity. Predictive validity refers to the ability of an instrument to predict outcome, i.e. response to radiotherapy or survival.

Clinical validity. Clinical validity refers to the ability of an instrument to discriminate between groups that are different regarding clinical features such as performance status and stage of disease.

Responsiveness. Responsiveness refers to the ability of an instrument to respond to changes in clinical status.

Another way of evaluating QoL instruments can be applied by testing the reliability, which refers to the ability of an instrument to minimise measurement errors. The reliability can be estimated by Cronbach's alpha coefficient ${ }^{52}$. Values exceeding 0.70 for the alpha coefficient are regarded as acceptable.

\section{Response formats}

Quality of life instruments usually employ different methods to quantify the answers of items addressed. A frequently used scale is the categorical or Likert scale. With this scale, the intensity or frequency of an item can be rated (Figure 1).

Figure 1: Example of a 4-point Likert-scale.

\begin{tabular}{|c|c|c|c|}
\hline 1 & 2 & 3 & 4 \\
\hline Not at all & A little & Quite a bit & Very much \\
\hline
\end{tabular}


The visual analogue scale (VAS) or linear analogue scale (LASA) consists of a horizontal line on which the patient can indicate the degree of an item by a vertical mark. Sometimes the extremes are described at the beginning and at the end (Figure 2).

Figure 2: Example of a Visual Analogue Scale.

Sometimes, a so-called numerical analogue scale is used, which is a kind of a cross between the Likert-scale and the VAS. On the numerical analogue scale, the patient can indicate the degree of an item by marking a number. Normally, the extremes are described at the beginning and at the end (Figure 3).

Figure 3: Example of a Numerical Analogue Scale

1

2
3
4
5
6 7

Very poor

Excellent

\section{Quality of life instruments}

QoL instruments can be grouped into several categories focusing on different populations. Initially, QoL instruments were developed to assess QoL in general populations, including the Sickness Impact Profile (SIP) ${ }^{53}$, the Nottingham Health Profile (NHP) ${ }^{54}$, the Dartmouth COOP Function Charts ${ }^{55}$, the EuroQoL ${ }^{56}$, and the Medical Outcomes Study 36-Item Short Form Health Survey (MOS SF-36) ${ }^{57}$.

The second category of QoL instruments have been developed applicable for groups of patients with a specific disease, the so-called disease-specific QoL instruments. For cancer patients, the most frequently used questionnaires are the European Organization for Research and Treatment of Cancer Quality of Life Questionnaire (EORTC QLQ-C36 and EORTC QLQ-C30) ${ }^{32,58}$, the Spitzer Quality of Life Index ${ }^{59}$, the Daily Diary Card (DDC) ${ }^{60}$, the Cancer Rehabilitation Evaluation System (CARES) ${ }^{61}$, the Rotterdam Symptom Checklist (RSCL) ${ }^{62}$, the Functional Living Index-Cancer (FLIC) ${ }^{63}$ and the Functional Assessment of Cancer Therapy-General Scale (FACT-G) ${ }^{64}$. 
The third categories of QoL instruments are those not only classified as disease-specific but also as site-specific. Now, three site-specific questionnaires are available specifically designed for lung cancer patients: the Lung Cancer Symptom Scale (LCSS), the EORTC QLQ-LC13 and the FACT-L.

\section{The Lung Cancer Symptom Scale}

The LCSS ${ }^{65-67}$ consists of two parts. The first part is designed to be used as self-reported questionnaire for the patients and the second part, which is optional, is designed for health care professionals. The patients' questionnaire focuses on physical and functional dimensions and contains 9 items including six major symptoms most relevant for lung cancer patients (appetite loss, fatigue, cough, dyspnea, haemoptysis and pain) and 3 summation items, measuring symptomatic distress, activity status and overall QoL. For the patients' questionnaire, a continuous response format was used by means of visual analogue scales. The observer instrument contains items regarding the six major symptoms, using a 5-point ordinal scale as response format. The LCSS proved to be a reliable and valid instrument to assess QoL in lung cancer patients. For the observer scale, high repeated interrater agreement has been reported ${ }^{65}$. Originally, the LCSS was developed as a short and practical tool in the assessment of QoL in clinical practice, but consequently, this is also one of its limitations. The LCSS has been criticised because it does not provide information concerning a number of important QoL dimensions. Secondly, it particularly measures disease-related QoL elements and no treatment-related factors, such as nausea and vomiting, hair loss and/or dysphagia. Therefore, this instrument is less suitable for comparison of treatment modalities that are likely to differ regarding treatment-induced toxicity. No Dutch version of the LCSS is available yet.

\section{The Functional Assessment of Cancer Therapy-Lung cancer instrument}

The FACT-L (version 4) ${ }^{64,68}$ is a self-reporting questionnaire consisting of two parts. The first part contains 27 items measuring general health-related dimensions including physical well-being, social/family well-being, emotional well-being and functional wellbeing. The second part is specifically designed for lung cancer patients and contains 9 items concerning frequently reported symptoms in lung cancer patients (i.e. dyspnea, weight loss, cough, appetite loss, chest pain and hair loss) and an item concerning 
smoking. In the most recent version (4.0), only 5-point Likert-scales are employed as response format. One of the advantages of the FACT-L is that it measures relative weight of importance attached to the components of QoL. The average time to complete the questionnaire is less than 10 minutes ${ }^{37}$. Although haemoptysis has been regarded as an important symptom by both patients and health professionals ${ }^{65}$, this symptom is not assessed with the FACT-L. Another limitation of this questionnaire is, in particular for studies investigating $Q o L$ in patients treated with radiotherapy, that a number of treatmentrelated items, such as dysphagia, are not assessed. Several studies reported good reliability, validity and responsiveness for the FACT- $L{ }^{64,69}$. The FACT- $L$ is available in many languages, including the Dutch language.

\section{European Organization for Research and Treatment of Cancer Quality of Life Questionnaire}

The EORTC QLQ-C30 ${ }^{32}$ (see Appendix 1) is a cancer-specific core questionnaire addressing various aspects of QoL. It contains five functional scales (physical functioning, role functioning, emotional functioning, cognitive functioning and social functioning), a global health/quality of life scale, 3 multi-item symptom scales and a number of single items addressing various symptoms and perceived financial impact. In version 1.0, as used in the study population which formed the base of this study as presented in this thesis, a yes/no response format was used for items referring to physical and role functioning, a 4-point Likert-scale for the other functioning and symptom scales and a 7point numerical analogue scale for the two items referring to the global health/quality of life scale. In the latest version, (3.0) all the yes/no formats have been replaced by 4-point Likert scales ${ }^{70}$. The EORTC QLQ-LC13 ${ }^{33}$ can be used as supplemental instrument to the EORTC QLQ-C30 and is lung cancer-specific. It contains 13 items, addressing the most frequently reported pulmonary symptoms, (i.e. cough, haemoptysis, dyspnea, pain in the chest, pain in the arm/shoulder and pain in other parts of the body) and a number of treatment-related items (sore mouth or throat, dysphagia, tingling hands or feet and hair loss). The response format is a 4-point Likert scale. The average time needed to complete the complete questionnaire including the lung cancer module is 11-12 minutes. Although there were some problems with patients' compliance with the first versions, high compliance rates have been reported with later versions ${ }^{71}$. The reliability, validity and 
responsiveness of these questionnaires have been confirmed in international studies ${ }^{32,33}$. One of the advantages of the EORTC instrument is that it contains a number of treatmentrelated items relevant to studies investigating new modalities in lung cancer. Furthermore, this instrument has been translated in many languages and validated for cross-cultural use ${ }^{33}$, which makes it applicable for collaborative international studies.

\section{Analysis of quality of life outcome}

Numerous reports have been published regarding the development, reliability and validity of QoL instruments. However, no consensus exists on how to analyse and present the data generated in clinical studies investigating changes in QoL and what should be regarded as response of symptoms and/or QoL. In the study population analysed for this thesis, the EORTC QLQ-C30 and EORTC QLQ-LC-13 were used and therefore the attention will be focused on the statistical analysis of these instruments.

The first step in the analysis is the conversion of the Likert scales of the individual items into scores of the different domains to which they belong. All scales all linearly converted to 0 to 100 scale. For functioning scales and the global health/QoL scale, higher scores represent higher levels of functioning. For the symptom scores, higher scores represent higher levels of symptomatology or problems ${ }^{70}$. For the single item scales, the conversion from Likert to linear scales means that only four values are possible (i.e. $0,33.3,66,7$ and 100). In general, in clinical studies on QoL, a large amount of data are generated, due to a varying number of domains and assessments before, during and after treatment.

Changes in QoL can be analysed in two different ways. In most studies, a group-based analysis is performed, comparing changes in the mean of scores of a given domain over time. Differences in mean scores between independent groups at one time point can be compared by means of a one-way analysis of variance, often referred to as a one-way ANOVA. The standard statistical method to deal with changes of mean scores over time is the repeated measures ANOVA. One of the main disadvantages of this method of analysis is that it only takes into account the cases with complete assessments at all time points. As QoL is particularly relevant in palliatively treated patient populations with relatively short survival times, the proportion of patients excluded from the analysis could 
be considerable and may lead to few patients left for a useful statistical analysis. Furthermore, the results could be biased when only those cases with longer survival times are taken into account. This problem is even more complicated when comparing two groups with different survival outcome. A number of methods have been proposed to overcome the problem of missing data ${ }^{72-76}$. For instance, Matthews and co-workers advocated to measure time to the first improvement from the baseline and/or time to first worsening from the baseline ${ }^{73}$. Another possible way to overcome the problem of missing data by death is the use of 'Time Without Symptoms and Toxicity (TWiST) or the Quality of life-oriented TWiST (Q-TWiST) ${ }^{75,76}$. Changes in the mean scores over time can also be evaluated by a modification of a repeated measurement ANOVA using a mixed effect modelling procedure. In contrast to a 'complete cases analysis' the mixed effect modelling retains in the analysis patients who dropout during follow up. With this kind of analysis, trends over time for dropouts and complete cases are estimated under the assumption that all patients within the same group have the same change pattern over time.

When large groups of patient are investigated, small differences may give statistically significant results. The question arises whether and to what extent these changes are of clinical relevance for individual patients. Osoba and co-workers investigated the significance for patients of changes in three functioning scales (physical, emotional and social functioning) and global QoL assessed with the EORTC QLQ-C30 ${ }^{77}$. The perceived changes in functioning and global QoL were assessed with the Subjective Significance Questionnaire (SSQ). In the SSQ, patients could rate their perception of change using a 7category scale ranging from 'much worse' through 'no change' to 'much better'. For patients who indicated 'no change', the mean change in score was not significantly different from 0 . In those who indicated 'a little', the mean change in scores varied between 5 and 10, for those who indicated 'moderate' between 10 and 20 and for those who indicated 'very much' change, greater than 20.

One of the disadvantages of analyses based on changes of mean scores, is that physicians and patients have trouble with the interpretation and translation of these results into what is clinically relevant for an individual patient. Both physicians and patients particularly want to know the probability of achieving relief of a given complaint. For instance, in a patient with severe pain referred for radiotherapy, the information that the 


\section{Chapter 1}

chance of getting significant relief of pain is approximately $60 \%$, is more clear than the information that the average decrease on a scale of 0 to 100 is approximately 20 points. Another frequently employed method to investigate $\mathrm{QoL}$ changes is to perform a subjectbased analysis, evaluating changes of a given domain in individual patients and classify these changes into response scores. In many studies, response of a symptom is defined as a decrease on a certain time point compared to what is measured at baseline. This approach implies that only those patients who have symptoms at baseline and who are still alive at the post-treatment assessment can be included in the analysis. The question arises again, whether the evaluable cases, who survived and suffered from complaints at baseline, are representative for the entire population investigated. This problem is even more pronounced in comparative studies investigating changes in QoL in patient populations with poor survival expectancy in particular when less frequently existing symptoms are considered relevant. Another disadvantage of this approach is that it only provides information of symptom relief at a certain time point. Some authors tried to overcome this problem by defining response as a decrease of symptoms on at least two or more assessments after treatment ${ }^{78,79}$. In some studies information was given of response rates at each time-point that assessments were made ${ }^{80}$. Others presented the duration of improvement of individual presenting symptoms as a proportion of survival time ${ }^{81}$ or used Kaplan-Meyer plots to estimate the frequency of palliation of individual symptoms by specified time points ${ }^{82,83}$.

In a recent paper, Stephens and co-workers presented a proposal for the standard definition of palliation of symptoms for use in the analysis of clinical trials ${ }^{84}$. In most studies, 4-point Likert scales are used for the scoring of symptoms at each assessment ${ }^{78-}$ $80,82,83$, classifying symptoms as absent, mild, moderate or severe. Stephens and coworkers pointed out that different criteria for palliation are required for patients presenting with different symptoms severity ${ }^{84}$, which can be referred to as improvement, control and prevention. Palliation of symptoms can be regarded as adequate in case of improvement by one or more categories. For those patients with mild symptoms, it makes sense to classify no worsening as response as well, i.e. control ${ }^{81}$. By classifying those patients without symptoms at baseline not getting worse as prevention, these patients can be included in statistical analyses as well ${ }^{86}$. 
It is extremely difficult to provide general rules how to perform and present analyses regarding QoL. In general, compliance should be reported and when relevant together with factors correlated with compliance ${ }^{87}$. Secondly, it makes sense to analyse data in different ways and to draw definitive conclusions only in case of consistency. Finally, Stephens recommended to set out predefined hypotheses, which also have the advantage of helping to choose the correct $\mathrm{QOL}$ instrument, deciding on the timing of administration and of calculating a suitable sample size ${ }^{84}$.

\section{Quality of life in inoperable NSCLC treated with external radiotherapy}

Taking into account the relatively poor results of radiotherapy in inoperable NSCLC, the palliative intent of treatment in many cases and the impact of lung cancer on psychosocial issues ${ }^{41,42,88}$, the number of reports addressing QoL questions in this category of patients undergoing treatment is surprisingly low. Studies in which QoL was examined by means of performance status, either the KPS, the ECOG or WHO performance status, were not included in this overview.

One of the first clinical studies reporting on QoL in inoperable NSCLC treated with radiotherapy was presented by Kaasa and co-workers ${ }^{89,90}$. In this randomised trial, previously untreated patients with inoperable NSCLC were randomised between radiotherapy (42 $\mathrm{Gy}$ in 15 fractions on the primary site and the regional lymph nodes) versus combination chemotherapy (cisplatin $70 \mathrm{mg} / \mathrm{m}^{2}$ and etoposide $100 \mathrm{mg} / \mathrm{m}^{2}$ given every 3 weeks with a maximum of 4 cycles). For the assessment of QoL a questionnaire was used covering four QoL issues including psychosocial well-being, physical function, clinical toxicity and every day activity. The median survival was 10 months for both groups. The overall tumour response rate was $25 \%$ for the radiotherapy patients and $15 \%$ for the chemotherapy patients. Nausea and vomiting and hair loss were more frequently reported after chemotherapy than after radiotherapy. However, dysphagia and a sore throat were more frequently observed after radiotherapy. No differences were noted regarding physical function and everyday toxicity. However, radiotherapy was superior with regard to psychosocial well-being and global QoL [Kaasa 1988]. 
The Medical Research Council (MRC) performed two randomised studies searching for the most optimal fractionation schedule for palliatively irradiated patients ${ }^{78,79}$. In the first MRC study ${ }^{78}$, conventional fractionation (FM regimen) consisting of $30 \mathrm{~Gy}$ in 10 fractions or $27 \mathrm{~Gy}$ in 6 fractions was compared with a two-fraction regimen (F2 regimen), consisting of 2 fractions of $8.5 \mathrm{~Gy}$ with a one week interval. Patients with inoperable NSCLC considered to advanced for curative or radical treatment were eligible. QoL was assessed by the physicians as well as by the patients using the MRC patient diary card ${ }^{91}$. Assessment of palliation by the clinicians included cough, haemoptysis, chest pain, anorexia, dysphagia, depression and anxiety. Assessments made by the patients included treatment-related toxicity such as nausea, vomiting and swallowing complaints and general health scales including activity, mood and overall condition. A total number of 369 eligible patients were randomised (184 F2, 185 FM). No difference in survival between the two treatment groups was observed. Furthermore, the results of the two treatment groups were similar regarding palliation of pulmonary symptoms and QoL. Based on these results, the regimen of two fractions of 8.5 Gy given one week apart was recommended.

In the second study ${ }^{79}$, the F2 regimen from the first study was compared with a onefraction regimen ( $\mathrm{F} 1$ regimen), consisting of a single fraction of $10 \mathrm{~Gy}$. The eligibility criteria were similar to the first MRC-study with the addition that patients also had to have a WHO performance status ranging from 2 to 4 and that their main symptoms had to be related to the primary intrathoracic tumour. QoL assessments were similar to the first study. A total number of 233 eligible patients were randomised (116 F2, 117 F1). Overall survival and the rates and duration of palliation were similar in both groups. However, patients treated with the $F 2$ regimen experienced significantly more dysphagia as compared to the patients treated with the F1 regimen. The authors recommended a single fraction of $10 \mathrm{~Gy}$ for patients with inoperable NSCLC with poor performance status.

Up to date, these three studies are the only ones in which QoL was investigated in inoperable NSCLC treated with radiotherapy alone. The study of Kaasa and co-workers was the only study using self-reported questionnaires in a multidimensional concept. In the two MRC-studies, only part of the assessments were made by the patients themselves. 


\section{$\square$ Quality of life after endobronchial brachytherapy}

Endobronchial brachytherapy is a form of radiotherapy allowing a short distance between the radiation source and the target volume and is usually used for endobronchial tumours in the proximal airways, i.e. the trachea, the main bronchus and the lobar bronchus. Afterloading brachytherapy involves insertion of a catheter down the tracheobronchial tree to the endobronchial tumour site. Thereafter, the catheter can be loaded with a radioactive source using an afterloading device, which has the advantage of eliminating radiation exposure to medical personnel. Nowadays, endobronchial brachytherapy is usually applied using an iridium-192 source, allowing delivery of a relatively high dose (5$20 \mathrm{~Gy}$ ) in a short time (5-10 minutes), often referred to as high dose rate brachytherapy (HDR). Inoperable NSCLC represent a difficult challenge for radiation-oncologists. The dose, that can be delivered with conventionally fractionated external radiotherapy, required to eliminate the tumour, exceeds the tolerance of normal structures such as the lung, the spinal cord and the heart. The main advantage of brachytherapy is the possibility of delivering a high dose of radiation to the local tumour, with selective sparing of the normal surrounding tissues, which would theoretically lead to enhancement of the therapeutic ratio. The addition of brachytherapy to external radiotherapy could therefore be beneficial in the management of selected tumours of the lung.

Patients with inoperable NSCLC frequently present with obstructive tumours in the proximal airways, leading to post-obstructive pneumonitis and atelectasis with subsequent symptomatology, such as dyspnea, cough and fever. The efficacy of external radiotherapy regarding to re-expansion of collapsed lung is limited. With external radiotherapy, reexpansion can be achieved in $21 \%$ to $61 \%$ of the cases ${ }^{92-94}$. With the combination of endobronchial brachytherapy and high dose external irradiation, the proportion of patients in which re-expansion is achieved appears to be higher compared to external irradiation alone varying from $67 \%$ to $99 \%{ }^{80,95,96}$. Retrospective studies suggest a beneficial effect of the combination of endobronchial brachytherapy regarding palliation of respiratory symptoms ${ }^{80,96}$, however, there are no clinical studies of the combination of brachytherapy and external radiotherapy which investigated changes in respiratory symptoms and QoL with validated instruments. 


\section{Chapter 1}

\section{$\square$ References}

1. Visser O, Coebergh JWW, Schouten LJ, van Dijck JAAM (Eds). Incidence of Cancer in the Netherlands 1996. Utrecht: Vereniging van Integrale kankercentra, 2000.

2. The World Health Organization: Histological Typing of Lung Tumours, second edtion. Am J Clin Pathol 77: 123-136, 1982.

3. Holmes EC. Surgical results and surgical adjuvant therapy for lung cancer. Am J Surg 143: 691-693, 1982.

4. Albain KS, Crowley JJ, LeBlanc M, Livingstone RB. Survival determinants in extensive-stage non-small cell lung cancer: the Southwest Oncology Group Experience. J Clin Oncol 1991; 9: 1618-1626.

5. Lanzotti VJ, Thomas DR, Boyle LE, Smith TL, Gehan EA, Samuels ML. Survival with inoperable lung cancer: an integration of prognostic variables based on simple clinical criteria. Cancer 1977; 39: 303-313.

6. Bonomi $P$, Gale M, Rowland $K$, et al. Pre-treatment prognostic factors in stage III non-small cell lung cancer patients receiving combined modality treatment. Int J Radiat Oncol Biol Phys 1991; 20: 247-52.

7. Stanley KE. Prognostic factors for survival in patients with inoperable lung cancer. J Natl Cancer Inst 65: 25-32, 1980.

8. Capewell S, Sudlow MF. Performance and prognosis in patients with lung cancer. Thorax 1990; 45: 951-956.

9. Sorensen JB, Badsberg JH, Olsen J. Prognostic factors in inoperable adenocarcinoma of the lung: a multivariate regression analysis of 259 patients. Cancer Res 49: 5748-5754, 1989.

10. Jeremic $B$, Shibamoto $Y$. Effect of interfraction interval in hyperfractionated radiotherapy with or without concurrent chemotherapy for stage III nonsmall cell lung cancer. Int J Radiat Oncol Biol Phys 1996; 34: 303-308.

11. Kupelian PA, Komaki $R$, Allen $P$, et al. Prognostic factors in the treatment of node-negative nonsmall cell lung carcinoma with radiotherapy alone. Int J Radiat Oncol Biol Phys 1996; 36: 607-613.

12. Nieder $C$, Nestle U, Ukena $D$, et al. Tumour markers as prognostic factors in non-small cell lung cancer. Strahlenther Onkol 171: 587-593, 1995.

13. PaterJL, Loeb M. Nonanatomic prognostic factors in carcinoma of the lung. Cancer 50: 326-331, 1982.

14. Ferrigno D, Buccheri GF. A comprehensive evaluation of serum ferritin levels in lung cancer patients. Lung Cancer 1992; 8: 85-94.

15. In Hermanek P and Sobin LH (Eds) In: TNM Atlas: Classification of malignant tumours; Springer-Verlag, Berlin 1992: 75-84.

16. Coy, $P$ and Kennelly, GM. The role of curative radiotherapy in the treatment of lung cancer. Cancer 1980; 45: 698702.

17. Dosoretz DE, Galmarini $\mathrm{D}$, Rubenstein $\mathrm{JH}$, et al. Local control in medically inoperable lung cancer: an analysis of its importance in outcome and factors determining the probability of tumor eradication. J Radiat Oncol Biol Phys 1993; 27: 507-516.

18. Gauden $S$, Ramsay $J$ and Tripciony $L$. The curative treatment by radiotherapy alone of stage I non-small cell lung cancer. Chest 1995; 108: 1278-1282.

19. Graham RH, Gebski VJ and Langlands $A O$. Radical radiotherapy for early nonsmall cell lung cancer. Int $J$ Radiat Oncol Biol Phys 1995; 31: 261-266.

20. Haffty BG, Goldberg NB, Gerstley J, et al. Results of radical radiation therapy in clinical stage I, technically operable non-small cell lung cancer. Int J Radiat Oncol Biol Phys 1988; 15: 69-73.

21. Kaskowitz $L$, Graham MM, Emami $B$, et al. Radiation therapy alone for stage 1 non-small cell lung cancer. $J$ Radiat Oncol Biol Phys 1993; 27: 517-523. 
22. Krol ADG, Aussems P, Noordijk EM, et al. Local irradiation alone for peripheral stage I lung cancer: could we omit the elective regional nodal irradiation? Int J Radiat Oncol Biol Phys 1996; 34: 297-302.

23. Noordijk EM, v.d. Poest Clement $F$, Hermans $J$, et al. Radiotherapy as an alternative to surgery in elderly patients with resectable lung cancer. Radiot Oncol 1988; 13: 83-89.

24. Sandler HM, Curran Jr WR and Tunisi III AT. The influence of tumor size and pre-treatment staging on outcome following radiation therapy alone for stage I non-small cell lung cancer. Radiat Oncol Biol Phys 1990; 19: 9-13.

25. Talton $B M$, Constable WC and Kersh $C R$. Curative radiotherapy in non-small cell carcinoma of the lung. Int $J$ Radiat Oncol Biol Phys 1990; 19: 15-21.

26. Zhang $\mathrm{HX}$, Yin WB, Zhang LJ, et al. Curative radiotherapy of early operable non-small cell lung cancer. Radiot Oncol 1989; 14: 89-94.

27. Slotman $\mathrm{BJ}$, Anthonisse IE and Njo KH. Limited field irradiation in early stage (T1-2NO) non-small cell lung cancer. Radiot Oncol 1996; 41: 41-44.

28. Cox JD, Azarnia N, Byhardt RW, et al. N2 (clinical) non-small cell carcinoma of the lung: Prospective trials of radiation therapy with total doses of 60 Gy by the Radiation Therapy Oncology Group. Int J Radiat Oncol Biol Phys 1991; 20: 7-12.

29. Curran WJ and Stafford PM. Lack of apparent differences in outcome between clinically staged IIIA and IIIB nonsmall cell lung carcinoma treated with radiotherapy. J Clin Oncol 1990; 8: 409-415.

30. Langendijk JA, de Jong JMA, Wanders SL, Slotman BJ. The revised international lung cancer staging system and the consequences for survival in patients with non-small cell lung cancer treated with radiotherapy. Radiat Oncol suppl, 1999.

31. Stewart LA and Pignon JP. Chemotherapy in non-small cell lung cancer: a meta-analysis using updated data on individual patients from 52 randomized clinical trials. BNIJ 1995; 311: 899-909.

32. Aaronson NK, Ahmedzai S, Bergman B, et al. The European Organization for Research and Treatment of Cancer QLQ-C30: a quality of life instrument for use in international clinical trials in oncology. J Natl Cancer Inst 1993; 85: 365-376.

33. Bergman B, Aaronson, NK, Ahmedzai S, et al. The EORTC QLQ-LC13: a modular supplement to the EORTC Core Quality of Life Questionnaire (QLQ-C30) for use in lung cancer clinical trials in oncology. Eur J Cancer 1994; 30A: 635-642.

34. Küchler $T H$, Schwartz $R$, Fletchner $H$, et al. Consensus on the implementation of quality of life assessments in oncological clinical trials. Eur J Cancer 1991; 27; 293-294.

35. Bradburn NM and Caplovitz D. Reports on Happiness. A pilot study of behavior related to mental health. Chigago: Aldine Publishing Compnay, 1965.

36. Aaronson, NK. Methodological issues in assessing the quality of life of cancer patients. Cancer 1991; 67 (suppl): 844-850.

37. Hollen PJ and Gralla RJ. Comparison of instruments for measuring quality of life in patients with lung cancer, Semin Oncol 1996; 23 (suppl 5): 31-40.

38. Karnofsky DA, Abelman WH, Craver LF, et al. The use of nitrogen mustards in palliative treatment of carcinoma. Cancer 1948; 1: 634-656.

39. Karnofsky DA and Burchenal JH. The clinical evaluation of chemotherapeutic agents in cancer. In: MacLeod CM, ed. Evaluation Chemotherapeutic Agenst. New York: Colombia University Press, 191-205, 1949.

40. Oken MM, Creech RH, Tormey DC, et al. Toxicity and response criteria of the Eastern Cooperative Oncology Group. Am J Clin Oncol 1982; 5: 649-655.

41. Bernhard J and Ganz PA. Psychosocial issues in lung cancer patients (part 1). Chest 1991; 99: 216-233. 


\section{Chapter 1}

42. Bernhard $J$ and Ganz PA. Psychosocial issues in lung cancer patients (part 2). Chest 1991; 99: 480-85.

43. De Haes JCJM. Quality of life: conceptual and theoretical considerations. In: Watson $M, G r e e r ~ S$, Thomas $C$, eds. Psychological oncology. Oxford: Pergamom Press, 61-70, 1988.

44. Till J. Use (and some possible abuses) of quality of life measures. In: Osoba D, ed.. Effect of cancer on quality of life. Bocca Raton, Florida: CRC Press: 137-154, 1991.

45. Hutchinson TA, Boyd NF and Feinstein AR. Scientific problems in clinical scales as demonstrated in the Kamofksy index of performance status. J Chron Dis 1979; 32: 661-666.

46. Slevin ML, Plant $H$, Lynch $D$, et al. Who should measure quality of life, the docter or the patient? Br J Cancer 1988; 57: 109-112.

47. Presant CA. Quality of life in cancer patients. Who measures what? Am J Clin Oncol 1984; 7: 751-757.

48. Ford $S$, Fallowfield $L$ and Lewis $S$. Can oncologists detect distress in their out-patients and how satisfied are they with their performance during bad news consultations. Br J Cancer 1994; 70: 676-770.

49. Osoba D. Lessons learned from measuring health-related quality of life in Oncology. J Clin Oncol 1994; 12: 608616.

50. Guyatt GH, Feeney DH and Patrick DL. Measuring health-related quality of life. Ann Intern Med 1993; 118: 517-524.

51. Michael M, Tannock IF. Measuring health-related quality of life in clinical trials that evaluate the role of chemotherapy in cancer treatment. Can Med Assoc J 1998; 58: 1727-1734.

52. Nunnaly JC, Bernstein IH. Psychometric therapy. New York: McGraw-Hill, 1994.

53. Bergner $M$, Bobbitt RA, Kressel $S$, et al. The Sickness Impact Profile: conceptual foundation and methodology for the development of a health status measure. Int J Health Serv 1976; 6: 393-415.

54. Hunt SM, McEwen J and McKenna SP. The Nottingham Health Profile User's Manual. 1981.

55. Nelson $E$, Wasson J, Kirk J, et al. Assessment of function in routine clinical practice: description of the COOP chart method and preliminary findings. J Chron Dis 1987; 40: 55S-63S.

56. The EuroQol Group. EuroQol-a new facility for the measurement of health-related quality of life. Health Policy 1990; 16: 199-208.

57. Ware JE, Shelbourne CD. A 36-item short-form health survey (SF-36): conceptual framework and item selection. Med Care 1992; 30: 473-483.

58. Aaronson NK, Bullinger $M$ and Ahmedzai S. A modular approach to quality-of-life assessment in cancer clinical trials. Recent Results in Cancer Res 1988; 111: 231-249.

59. Spitzer WO, Dobson AJ, Hall J, et al. Measuring the quality of life of cancer patients. J Chron Dis 1981; 34: 585597.

60. Fayers PM, Bleehen NM, Girling DJ, et al. Assessment of quality of life in small-cell lung cancer using a daily diary card developed by the Medical Research Council Lung Cancer Working Party. Br J Cancer 1991; 64: 299-306.

61. Ganz PA, Schag CAC, Lee JJ and Sim MS. The CARES: A generic measure of health-related quality of life for patients with cancer. Quality Life Res 1992; 1: 19-29.

62. De Haes JCJM, van Knippenberg FCE and Neijt JP. Measuring psychological and physical distress in cancer patients: Structure and application of the Rotterdam symptom checklist. Br J Cancer 1990; 62: 1034-1038.

63. Schipper $\mathrm{H}$, Clinch J, McMurray A, et al. Measuring the quality of life of cancer patients. The Functional Living Index-Cancer: Development and validation. J Clin Oncol 1984; 2: 472-483.

64. Cella DF, Tulsky DS, Gray G, et al. The functional Assessment of Cancer Therapy Scale: Development and validation of the general measure. J Clin Oncol 1993; 11: 570-579.

65. Hollen PJ, Gralla RJ, Kris MG. et al. Quality of life assessment in individuals with lung cancer: testing the Lung Cancer Symptoms Scale (LCSS). Eur J Cancer 1993; 29A (suppl 1):S51-58. 
66. Hollen PJ, Gralla RJ and Kris MC. Measurement of quality' of life in patients with lung cancer in multicentre trial of new therapies: psychometric assessment of the Lung Cancer Symptom Scale. Cancer 1994; 73: 2087-2098.

67. Hollen PJ, Gralla RJ, Kris MG and Cox C. Quality of life during clinical trials: Conceptual model for the Lung Cancer Symptom Scale (LCSS). Support Care Cancer 1994; 2:213-222.

68. Cella DF, Bonomi AE, Lloyd SR, et al. Reliability and validity of the Functional Assessment of Cancer Therapy-Lung (FACT-L quality of life instrument. Lung Cancer 1995; 12:199-220.

69. Yellen SB, Cella DF, Webster $K$, et al. Measuring fatigue and other anemia-related symptoms with the Functional Assessment of Cancer Therapy measurement system. J Pain Symptom Manage 1997; 13: 63-74.

70. Fayers PM, Aaronson NK, Bjordal K, et al. EORTC QLQ-C30 Scoring Manual, 2nd Edition, 1999.

71. Sadura A, Pater J, Osoba D, et al. Quality of life assessment: Patient compliance with questionnaire completion. J Natl Cancer Inst 1992; 84: 1023-1026.

72. Zwinderman AH. Statistical analysis of longitudinal quality of life data with missing measurements. Qual Life Res 1991; 1: 219-224.

73. Matthews, JNS. A refinement to the analysis of serial data using summary measures. Stats in Medicine 1993; 12: 27-37.

74. Torrance GW. Health status index models: a unified view. Management Sci 1987; 22: 990-1001.

75. Gelber RD and Goldhirsch A. New endpoint for the assessment of adjuvant therapy in postmenopausal women with inoperable breast cancer. J Clin Oncol 1986; 4: 1772-1779.

76. Feldstein ML. Quality-of life adjusted survival comparing cancer treatments: A commentary on TWiST and QTWiST, Cancer 1991; 67: 851-854.

77. Osoba $D$, Rodrigues $G$, Myles $\mathrm{J}$, et al. Interpreting the significance of changes in quality of life scores. $\mathrm{J}$ Clin Oncol 1998; 16: 139-144.

78. Bleehen NM, Girling DJ, Fayers PM, et al. Inoperable non-small lung cancer (NSCLC): a Medical Research Council randomized trial of palliative radiotherapy with two fractions or 10 fractions. $\mathrm{Br} J$ Cancer 1991; 63: 265-270.

79. Bleehen NM, Girling DJ, Machin D, et al. A Medical Research Council (MRC) randomized trial of palliative radiotherapy with two fractions or single fraction in patients with inoperable non-small-cell lung cancer (NSCLC) and poor performance status. Br J Cancer 1992; 65: 934-941.

80. Speiser BL and Spratling L. Remote afterloading brachytherapy for the local control of endobronchial carcinoma. Int J Radiat Oncol Biol Phys 1993; 25: 579-587.

81. Muers MF and Round CE. Palliation of symptoms in non-small cell lung cancer: a study by the Yorkshire Regional Cancer Organisation Thoracic Group. Thorax 1993; 48: 339-343.

82. Medical Research Council Lung Cancer Working Party. Randomized trial of palliative two-farction versus more intensive 13-fraction radiotherapy for patients with inoperable non-small cell lung cancer and good performance. $\mathrm{Br}$ J Cancer 1996; 73: 406-413.

83. Medical Research Council Lung Cancer Working Party. Randomized trial of four-drug versus less intensive twodrug chemotherapy in the palliative treatment of patients with small cell lung cancer (SCLC) and poor prognosis. $\mathrm{Br}$ J Cancer 1996; 73: 413-417.

84. Stephens RJ, Hopwood P and Girling DJ. Defining and analysing symptom palliation in cancer clinical trials: a deceptively difficult exercise. Br J Cancer 1999; 79: 538-544.

85. Sur RK, Kochbar R and Machin D. Oral sucralfate in acute radiation oesophagitis. Acta Oncol 1994; 33: 61-63.

86. Van Holten-Verzantvoort ATM, Kroon HM, Bijvoet OLM, et al. Palliative pamidronate treatment in patients with bone metastases from breast cancer. $J$ Clin Oncol 1993; 11: 491-498. 
87. Hopwood F, Stephens RJ and Machin D. Approaches to the analysis of quality of life data: experiences gained from a medical Research council lung cancer working party palliative chemotherapy trial. Qual Life Res 1994; 3: 339-352.

88. Bernhard J and Ganz PA. Psychosocial issues in lung cancer patients. Cancer Treat Res 1995; 72:363-390.

89. Kaasa $S$, Mastekaasa $A$ and Thorud $F$. Toxicity, physical function, and everyday activity reported by patients with inoperable non-small cell lung cancer in a randomized trial: Acta Oncol 1988; 27: 343-349.

90. Kaasa S, Mastekaasa $A$ and Naess $S$. Quality of life of lung cancer patients in a randomized controlled clinical trial evaluated by a psychosocial well-being questionnaire. Acta Oncol 1988; 27: 335-342.

91. Fayers PM and Jones DR. Measuring and analysing quality of life in cancer clinical trials. Stat Med 1983; 2: 429438.

92. Majid $O A$, Lee $S$, Khushalani $S$ and Seydel HG. The response of atelectasis from lung cancer to radiation therapy. Int J Radiat Oncol Biol Phys 1986; 12: 231-232.

93. Chetty KG, Moran EM, Sassoon CSH, et al. Effect of radiation therapy on bronchial obstruction due to bronchogenic carcinoma. Chest 1989; 95: 582-584.

94. Slawson RG and Scott RM. Radiation therapy in bronchogenic carcinoma. Ther Radiol 1979; 132: 175-176.

95. Bastin KT, Mehta MP and Kinsella TJ. Thoracic volume radiation sparing following endobronchial brachytherapy: a quantitative analysis. Int J Radiat Oncol Biol Phys 1993; 25: 703-707.

96. Chang LL, Horvath J, Peyton $W$ and Ling $S$. High dose rate afterloading intraluminal brachytherapy in malignant airway obstruction of lung cancer. Int J Radiat Oncol Biol Phys 1994; 28(3): 589-596. 
Chapter 2

Aims of this thesis 


\section{口 Rationale}

As pointed out in the introduction, it may be quite clear that little information exists regarding QoL assessed with validated multidimensional instruments in patients with inoperable NSCLC treated with radiotherapy. Information regarding QoL is extremely relevant for this category of patients because of (1) the generally poor prognosis even in curatively treated patients, (2) the fact that most patients are treated with the aim of palliation and improvement of QoL and (3) the possible negative effects of radiationinduced toxicity on QoL. Although, overall survival remains of primary interest, it should be interpreted in the light of symptom palliation and overall QoL because the side effects of radiotherapy may negate the assumed benefit of improved survival. Furthermore, information about baseline QoL and QoL outcome after treatment may provide important information for the design of and the calculation of the number of patients required for future randomised trials, in which $\mathrm{QoL}$ is considered to be a relevant endpoint.

\section{aims of the thesis}

The aims of this thesis are:

1. To describe QoL of patients with inoperable NSCLC before and after radiotherapy.

2. To evaluate the prognostic significance of pre-treatment QoL among patients with inoperable NSCLC treated with radiotherapy.

3. To determine which factors affect the changes in QoL as observed after radiotherapy.

4. To determine the safety and value of the addition of endobronchial brachytherapy to external irradiation as primary treatment of centrally localised inoperable NSCLC.

\section{Design of the thesis}

For the purpose of this thesis, two prospective studies were initiated in the Radiotherapeutisch Instituut Limburg (RTIL). The first prospective study (RTIL-9401) was open from March 1994 to November 1996. Patients with inoperable NSCLC referred to the 
RTIL for primary radiotherapy on the intrathoracic tumour could be included. The patients were divided into three prognostic groups (Figure 1). In case of lymph node metastases in the supraclavicular regions, the presence of distant metastases and/or a WHO performance status $>2$, patients were selected for palliative radiotherapy (30 Gy in 10 fractions). Those patients with stage I or II disease with a maximum diameter of 4 centimetre and a WHO performance status $0-2$ were selected for curative radiotherapy (70 $\mathrm{Gy}$ in 35 fractions). The remaining patients were selected for radical radiotherapy (60 Gy in 24 fractions).

Figure 1: Selection criteria for the different fractionation schedules

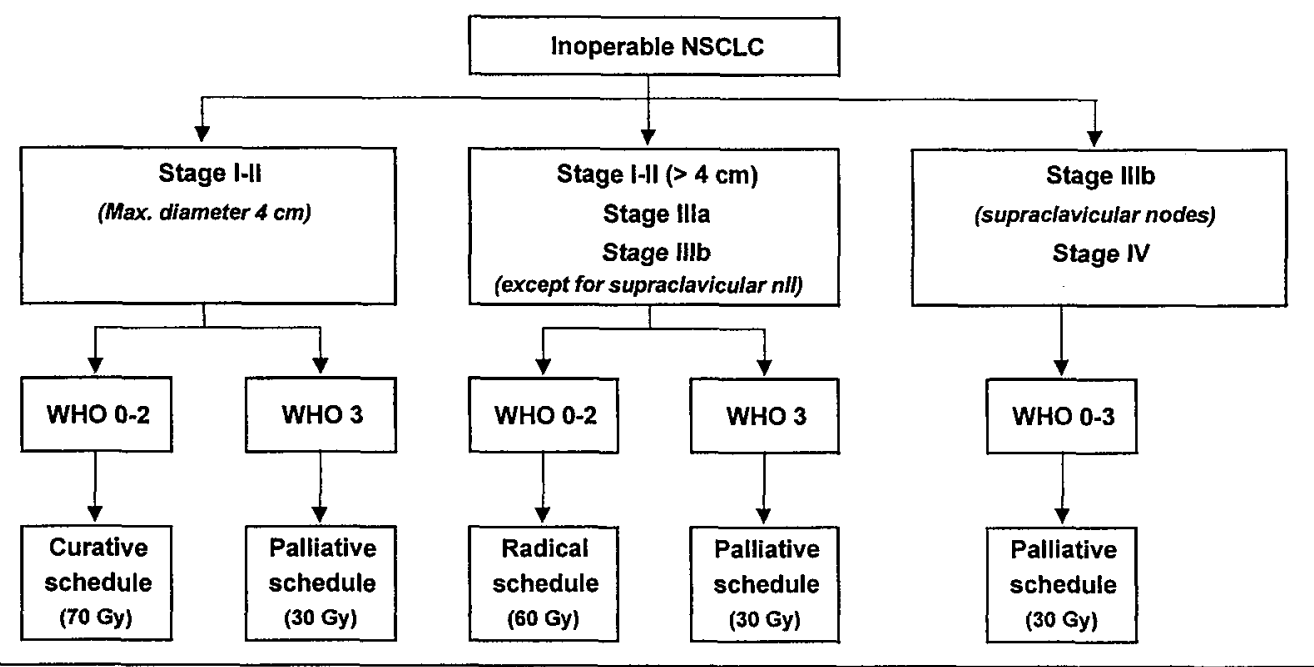

In the aforementioned period, 302 patients were referred for primary radiotherapy for inoperable NSCLC. Twenty patients $(7 \%)$ were excluded because the diagnosis could not be confirmed pathologically. Another 13 patients (4\%) were excluded because there was no baseline QoL assessment due to administrative failures. Five patients (2\%) refused to participate in the study. The remaining 264 patients (87\%) formed the base of this study, of which 35 patients (13\%) were treated according to the curative schedule, 164 patients (62\%) according to the radical schedule and 65 patients (25\%) according to the palliative schedule. In all these patients, QoL was assessed before radiotherapy and 2 weeks, 6 weeks, 3,6 , and 12 months after radiotherapy. In the group of patients treated according 
to the radical and curative schedule, a QoL assessment was made during radiotherapy as well.

Figure 2: Design of the thesis.

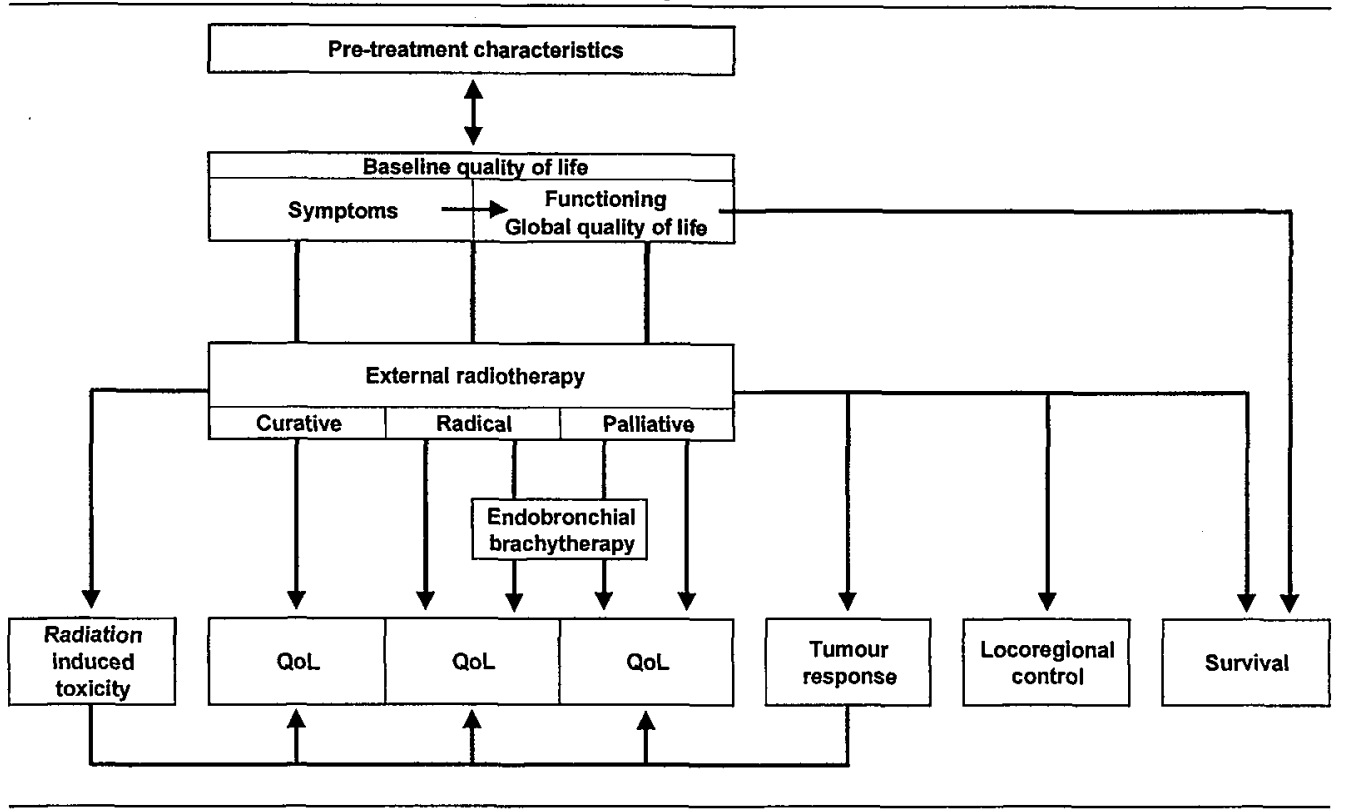

The second study (RTIL-9403/CKVO-9506) was a prospective randomised multicentre trial which was supported by the Dutch Cancer Society (KWF) and the Comprehensive Cancer Centre Limburg (IKL). In this study, patients with centrally localised NSCLC were randomly assigned to receive external radiotherapy alone or external radiotherapy plus endobronchial brachytherapy. In this study, QoL was the main endpoint and assessed using the EORTC instruments. The design of the thesis is outlined in Figure 2.

In the first part of the thesis, the attention is focused on baseline QoL. The association between pre-treatment characteristics with baseline $Q o L$ is described in chapter 3 . In this chapter the effect of respiratory symptoms on functioning and global QoL is also presented. The prognostic significance on the overall survival of baseline $\mathrm{QoL}$ is described in chapter 4 .

One of the objectives was to assess which factors influenced the changes in QoL observed after radiotherapy, such as objective tumour response. To investigate whether 
the chest radiograph is a reliable tool in the assessment of tumour response after radiotherapy in NSCLC and to investigate the interobserver variability of both chest radiography and $\mathrm{CT}$ scan of the chest a prospective evaluation was performed. The results of this study are described in chapter 5 .

The changes of QoL after palliative, radical and curative radiotherapy are reported in chapter 6,7 and 8 respectively.

A retrospective study was performed to investigate whether endobronchial brachytherapy is a risk factor for massive haemoptysis before starting a prospective randomised study. The results of this retrospective analysis are described in chapter 9 . Chapter 10 describes the results of a prospective randomised study investigating the value of the addition of endobronchial brachytherapy to external irradiation, particularly with regard to QoL outcome. 
Chapter 3

\title{
Pre-treatment quality of life of inoperable non-small cell lung carcinoma patients referred for primary radiotherapy.
}

\author{
Hans Langendijk \\ Neil Aaronson \\ Guul ten Velde \\ Jos de Jong \\ Martin Muller \\ Miel Wouters
}


Chapter 3

\section{a Abstract}

This study was performed to examine the association between the most important prognostic factors in NSCLC and self-reported pre-treatment quality of life (QoL) and the impact of the presence, severity and changes of respiratory symptoms on general symptoms and QoL. Two hundred and sixty-two patients were included in study. The EORTC QLQ-C30 and EORTC QLQ-LC13 were used to assess symptoms and QoL before radiotherapy.

Patients with inoperable NSCLC show major differences in self-reported QoL as a function of clinical prognostic factors. A significant association was found between WHO performance status and QoL but not with other prognostic factors. Dyspnea was the only respiratory symptoms associated significantly with general symptoms, physical and psychosocial functioning and QoL. Furthermore, changes in dyspnea were associated significantly with changes in physical and role functioning, global QoL and fatigue as assessed 6 weeks after radiotherapy. These results indicate that palliation of dyspnea may have a significant beneficial effect on QoL and that palliation of other respiratory symptoms is not necessarily associated with improvement of general symptoms, physical and psychological functioning or global QoL. 


\section{Introduction}

Lung cancer is the leading cause of cancer-related death. Approximately $75 \%$ of these patients are diagnosed with non-small cell lung carcinoma (NSCLC). For these patients, surgery is presently the best treatment for stage I and II disease (UICC 1997) and might play a role in selected cases with stage Illa disease. Patients with NSCLC may be inoperable for several reasons, including advanced loco-regional tumour extension, the presence of distant metastases and/or co-morbidity. Many of these inoperable patients are treated by radiotherapy with or without chemotherapy.

Patients' self-reported QoL has become an important endpoint for treatment comparisons in many clinical trials. For this purpose, a number of methods have been developed to allow reliable and valid measurement of various aspects of $\mathrm{QoL}{ }^{1-4}$. It is generally believed that patients with good prognosis experience a higher level of health-related QoL compared to patients with poor prognosis. In general, prognosis in cancer depends on factors such as disease extension, performance status and weight loss. Aaronson et al. found that lung cancer patients with poor values on these indicators experienced significantly lower QoL compared to those with higher values ${ }^{5}$. Cella et al. examined the relationship between extent of disease, performance status and psychological distress in a cohort of patients with small cell lung cancer ${ }^{6}$. They found that performance status correlated significantly with psychological distress but that the extent of disease correlated significantly with psychological distress only for the patients with poor performance status. Ringdal et al. investigated the differences in QoL between patients with good, intermediate and poor prognosis ${ }^{7}$. They found that prognosis was moderately related to physical aspects of QoL but not to social and psychological well being. In this study, patients with a range of cancer diagnosis were involved. These results indicate that the presumed association between QoL, prognosis and individual prognostic factors is far from consistent. In particular, this association has never been documented among patients with inoperable NSCLC referred for radiotherapy.

Most patients with inoperable NSCLC are irradiated to improve loco-regional tumour control and to palliate respiratory symptoms. It is also generally believed that a relationship exists between respiratory symptoms and general aspects of QoL, and that palliation of these symptoms leads to improvement of QoL. To our knowledge, this putative association has never been studied. 
The first aim of this study was to examine the pre-treatment QoL characteristics, as assessed by the EORTC QLQ-C30, of patients with NSCLC referred for radiotherapy, classified into three different prognostic groups. Second, we examined the impact of the most important prognostic factors in NSCLC and the impact of the presence and severity of respiratory symptoms on general symptoms and QoL before radiotherapy. Third, we evaluated the association between changes in local symptoms and changes in general symptoms, functioning and global QoL.

Four hypotheses were tested in this analysis. First, we expected QoL in the poor prognostic groups to be worse compared to the groups with better prognosis. Second, we expected that self-reported QoL would be significantly better among patients with high versus low performance status, limited versus extended disease, and limited versus substantial weight loss. Third, we hypothesised that the severity of respiratory symptoms, as assessed by the supplementary QLQ-LC13 questionnaire, would be associated negatively with patients' levels of self-reported physical and psychological functioning, and with their experience of more general symptoms such as fatigue. Finally, we expected that improvement of respiratory symptoms, as assessed by the QLQ-LC13 questionnaire after radiotherapy, would be associated positively with improvement of the more general symptoms, physical and psychological functioning, and with global QoL.

\section{Material and methods}

\section{Patients}

The study sample included patients with inoperable NSCLC who were to receive primary external irradiation only of the primary tumour site and/or regional lymph nodes. Other eligibility criteria included WHO performance status score $\leq 3$, good command of the Dutch language, no prior chemotherapy, no prior surgery, no prior radiotherapy, and no signs of brain metastases.

To assess the extent of disease, physical examination, chest radiography, computed tomography of the chest including the liver and adrenal glands and bronchoscopy were performed on all patients. Computed tomography of the brain and bone scintigraphy was not performed routinely, but only in case of suspicion of brain or bony metastases. WHO performance status and weight loss were assessed prospectively. 
From June 1994 to December 1996, 262 patients were entered in the study and completed a baseline QoL questionnaire. There were 36 females' (14\%) and 226 males $(86 \%)$, with a median age of 69 years $(37-88$ years).

\section{Prognostic groups}

Patients with a WHO performance status 3 , supraclavicular lymph node metastases and/or distant metastases with symptoms related to intrathoracic tumour, were considered to have the worst prognosis and were selected for the palliative fractionation schedule. These patients were treated with a total dose of 30 Gy using fraction doses of 3 Gy (4 times a week).

Patients with stage I or II disease (UICC 1992) with a tumour diameter $>4$ centimetre or stage IIla and stage IIIb disease (UICC 1992) without supraclavicular lymph node metastases and a WHO performance $\leq 2$ were considered to have an intermediate prognosis and were selected for the radical fractionation schedule. These patients were treated to a total dose of $60 \mathrm{~Gy}$ in 6 weeks.

In case of stage I or II disease (UICC 1992) with a maximum diameter of 4 centimetre and a WHO performance $\leq 2$, the curative fractionation schedule was selected. These patients were treated to a total dose of $70 \mathrm{~Gy}$ in 7 weeks.

In the present study, 35 patients $(13 \%)$ were selected for the curative schedule, 142 patients $(54 \%)$ for the radical schedule and 65 patients $(25 \%)$ for the palliative schedule.

\section{Quality of life assessment}

Quality of life was assessed by means of the EORTC core questionnaire, the QLQ-C30, and the supplemental lung cancer specific module, the QLQ-LC13. The QLQ-C30 (version 1.0) contains 30 questions. The questionnaire is organised into five functional scales (physical (PF), role (RF), emotional (EF), cognitive (CF) and social (SF)), three symptoms scales (fatigue, pain and nausea and vomiting), a global health/quality of life scale, and a number of single items assessing additional symptoms (dyspnea, sleep disturbance, constipation and diarrhoea) and perceived financial impact. This questionnaire has proven to be a valid and reliable tool when used among a wide range of cancer patient populations, including lung cancer patients '. It has been translated and validated for use among Dutch patients. The QLQ-LC13 contains 13 questions concerning symptoms frequently present in lung 


\section{Chapter 3}

cancer patients and treatment related symptoms. This supplemental questionnaire has proven to be a valid and reliable tool among lung cancer patients ${ }^{8}$.

The questionnaires were administered approximately 1 week before the start of radiotherapy, and at regular intervals during and after the course of treatment. This paper focuses primarily on the pre-treatment assessment. To investigate the association between changes over time in respiratory symptoms and in other more general symptoms and QoL, limited use is made of the follow-up data, i.e. the assessment at 6 weeks post-radiotherapy. Prior to participation in the study, patients were informed by the radiation-oncologist about the seriousness of their illness, the prognosis and the aims of treatment. Informed consent was obtained from all patients according to local ethical standards.

\section{Statistics}

All scales of the QLQ-C30 and QLQ-LC13 were linearly converted to a 0 to 100 scale. For the functional and global health status/quality of life scales, higher scores represent a better level of functioning. For symptom scales, higher scores represent a greater degree of symptoms. For the purpose of this study only the general symptom scales assessing the most relevant symptoms for lung cancer patients were included in the analysis, i.e. fatigue and appetite loss. With regard to respiratory symptoms, baseline scores for cough, haemoptysis, dyspnea, dysphagia, pain arm/shoulder and pain in the chest wall were analysed. For the single-item scales (cough, haemoptysis, dysphagia, pain arm/shoulder, pain chest wall), four ranges of scores were defined: $1=$ nil, $2=$ mild, $3=$ moderate and $4=$ severe. For the multi-item scale (dyspnea), four ranges of scores were defined as well, based on the converted score ranging from 0 to 100 : $0=$ nil, 1 to $34=$ mild, 35 to $67=$ moderate and 68 to $100=$ severe.

To analyse the association between the clinical prognostic factors and QoL, the general linear model (GLM) multivariate analysis of variance (MANOVA) was used. The GLMMANOVA approach can be used to test the hypothesis of a significant association between a set of interrelated dependent variables, e.g. the QoL scales, and one or more grouping variables. This method was preferred over analysis of each QoL scale separately, because it also takes into account the correlation among the individual QoL scales. This multivariate approach also protects against type I errors. In the present study, the GLM-MANOVA was performed in two steps. First, to investigate the association of a given prognostic factor with QoL, an overall analysis was performed to establish whether the prognostic factor 
was associated significantly with any of the QoL measures. Wilks' lambda (often referred to as the U-statistic) was used to test the impact of each prognostic variable included in the multivariate model on QoL. Wilks' lambda can take on values ranging between 0 , indicating large differences in group means, and 1, indicating no differences in group means. In case of a significant association between a prognostic factor and all QoL scales taken together, a second analysis was performed to investigate the association between that factor and each QoL scale separately.

The variance in QoL scores sustained by a given prognostic factor is expressed by the effect-size (explained variance). For example, an effect-size for a prognostic factor of 0.18 indicates that $18 \%$ of the variance in QoL scores is accounted for by the prognostic factor, being evaluated.

To investigate the association between changes in respiratory symptoms and general symptoms, physical and psychological functioning and global QoL, the same multivariate procedure was carried out. For this purpose, the change scores between baseline and 6 weeks follow up were calculated. The change scores for the respiratory symptoms were organised into three categories: worse (change score $>5)$, no change $(-5 \leq$ change score $\leq 5$ ) or improved (change score $<-5$ ).

\section{Results}

\section{Prognostic groups and survival}

The patients' pre-treatment characteristics, stratified by prognostic groups are presented in Table 1. Although weight loss was not taken into account in the selection of one of the three prognostic groups, significant differences concerning this prognostic factor were observed between the three groups. This difference was not only observed between the palliative group and the other two groups $(p<0.001)$, but also between the radical and the curative group $(p=0.008)$. As expected, performance status was significantly lower in the palliative group than in the other two groups $(p<0.001)$. The difference in performance status noted between the radical and curative group was not significantly different.

The median survival was 19.1 months (95\%-ci: 15.4-22.9) for the curative group, 8.5 months (95\%-ci: 6.7-10.4) for the radical group and 4.1 months (95\%-ci: 2.4-5.8) for the palliative group (Figure 1). 
Chapter 3

Table 1. Pre-treatment characteristics stratified by the selected treatment schedule.

\begin{tabular}{|c|c|c|c|c|}
\hline Variables & $\begin{array}{c}\text { Curative } \\
\text { n (\%) }\end{array}$ & $\begin{array}{c}\text { Radical } \\
\mathrm{n}(\%)\end{array}$ & $\begin{array}{c}\text { Palliative } \\
\text { n (\%) }\end{array}$ & $\begin{array}{c}\text { All patients } \\
n\end{array}$ \\
\hline \multicolumn{5}{|l|}{ T-classification } \\
\hline $\mathrm{T} 1$ & $17(49 \%)$ & $12(7 \%)$ & $2(3 \%)$ & $31(12 \%)$ \\
\hline T2 & $18(51 \%)$ & $59(36 \%)$ & $20(31 \%)$ & $96(37 \%)$ \\
\hline T3 & 0 & $28(17 \%)$ & $11(17 \%)$ & $39(15 \%)$ \\
\hline T4 & 0 & $65(40 \%)$ & $32(49 \%)$ & $96(37 \%)$ \\
\hline \multicolumn{5}{|l|}{$\mathrm{N}$-classification } \\
\hline No & $35(100 \%)$ & $53(32 \%)$ & $14(22 \%)$ & $102(39 \%)$ \\
\hline N1 & 0 & $3(2 \%)$ & 0 & $3(1 \%)$ \\
\hline N2 & 0 & $86(52 \%)$ & $29(45 \%)$ & $113(43 \%)$ \\
\hline N3 & 0 & $22(13 \%)$ & $22(34 \%)$ & $44(17 \%)$ \\
\hline \multicolumn{5}{|l|}{ Stage (UICC 1992) } \\
\hline Stage 1 & $35(100 \%)$ & $18(11 \%)$ & $2(3 \%)$ & $55(21 \%)$ \\
\hline Stage II & 0 & $2(1 \%)$ & 0 & $2(1 \%)$ \\
\hline Stage Illa & 0 & $65(40 \%)$ & $9(14 \%)$ & $73(28 \%)$ \\
\hline Stage IItb & 0 & $79(48 \%)$ & $37(57 \%)$ & $115(44 \%)$ \\
\hline Stage IV & 0 & 0 & $17(26 \%)$ & $17(7 \%)$ \\
\hline \multicolumn{5}{|l|}{ Tumour type } \\
\hline Squamous cell carcinoma & $23(66 \%)$ & $95(58 \%)$ & $32(49 \%)$ & $149(57 \%)$ \\
\hline Adeno carcinoma & $2(6 \%)$ & $28(17 \%)$ & $12(19 \%)$ & $42(16 \%)$ \\
\hline Undifferentiated carcinoma & $10(29 \%)$ & $41(25 \%)$ & $21(32 \%)$ & $71(27 \%)$ \\
\hline \multicolumn{5}{|l|}{ Weight loss } \\
\hline none & $30(86 \%)$ & $76(47 \%)$ & $21(33 \%)$ & $127(49 \%)$ \\
\hline $1-10 \%$ & $2(6 \%)$ & $54(33 \%)$ & $19(30 \%)$ & $74(29 \%)$ \\
\hline$>10 \%$ & $3(9 \%)$ & $3(20 \%)$ & $23(38 \%)$ & $58(22 \%)$ \\
\hline unknown & 0 & 3 & 1 & 3 \\
\hline \multicolumn{5}{|l|}{ Performance status } \\
\hline WHOO & $20(57 \%)$ & $63(38 \%)$ & $6(9 \%)$ & $87(33 \%)$ \\
\hline WHO 1 & $13(37 \%)$ & $78(48 \%)$ & $21(32 \%)$ & $112(43 \%)$ \\
\hline WHO 2 & $2(6 \%)$ & $23(14 \%)$ & $16(25 \%)$ & $41(16 \%)$ \\
\hline WHO 3 & 0 & 0 & $22(34 \%)$ & $22(8 \%)$ \\
\hline
\end{tabular}




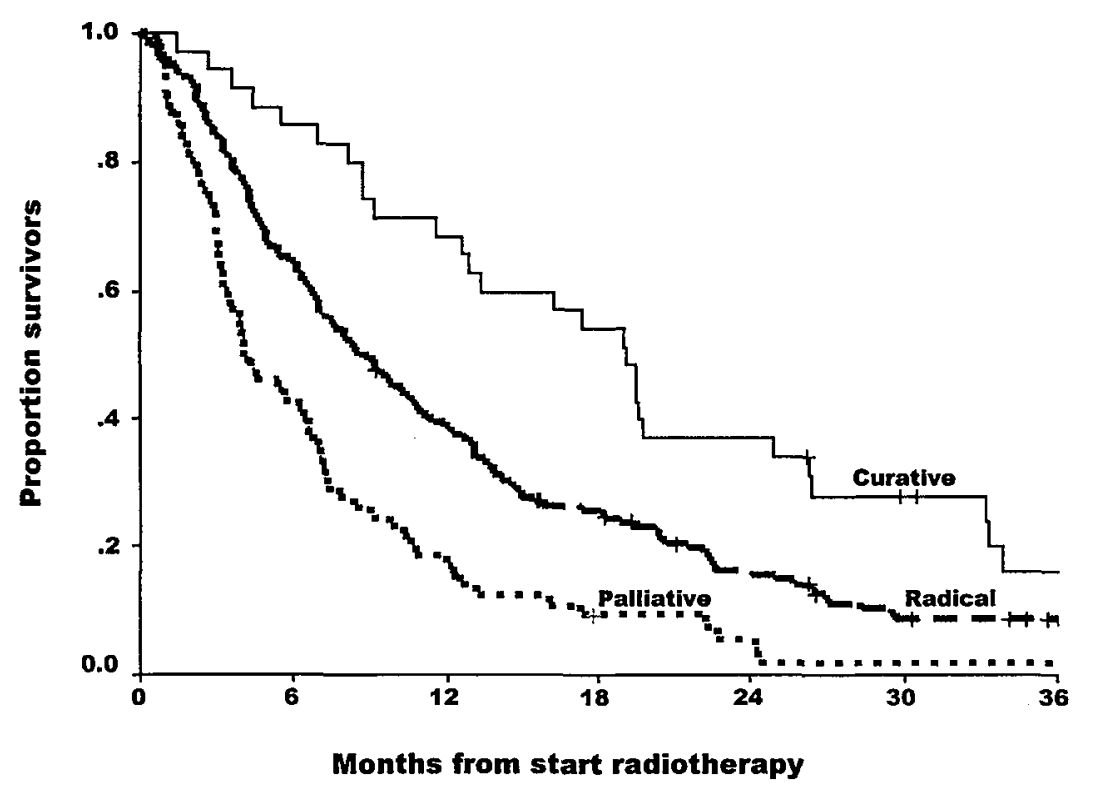

Prognostic groups and baseline quality of life

The mean baseline QoL-scores stratified by prognostic groups are shown in Figure 2. In general, the baseline scores for the functioning scales and global QoL scale of those selected for curative treatment were slightly higher than the scores observed in those selected for radical radiotherapy. However, these differences were not statistically significant. For the symptom scales, a significant difference in baseline scores between the aforementioned two treatment groups was observed for appetite loss $(p=0.003)$. The mean score for appetite loss was 8.6 (SE 3.4) in the curative group and 27.1 (SE 2.7) in the radical group. No significant differences between these two groups were observed for the other general symptoms or for any of the respiratory symptoms.

In the palliative group, the mean baseline scores for all functioning scales and the global QoL scale were significantly lower compared to those observed in the curative and radical groups. Furthermore, the mean baseline scores for the general and respiratory symptoms in the palliative group were significantly higher than those observed in the radical and curative group, with the exception of insomnia, cough and pain in the arm/shoulder. 
Figure 2: Baseline scores for symptoms and quality of life by prognostic groups

\section{Prognostic groups and functioning scales}

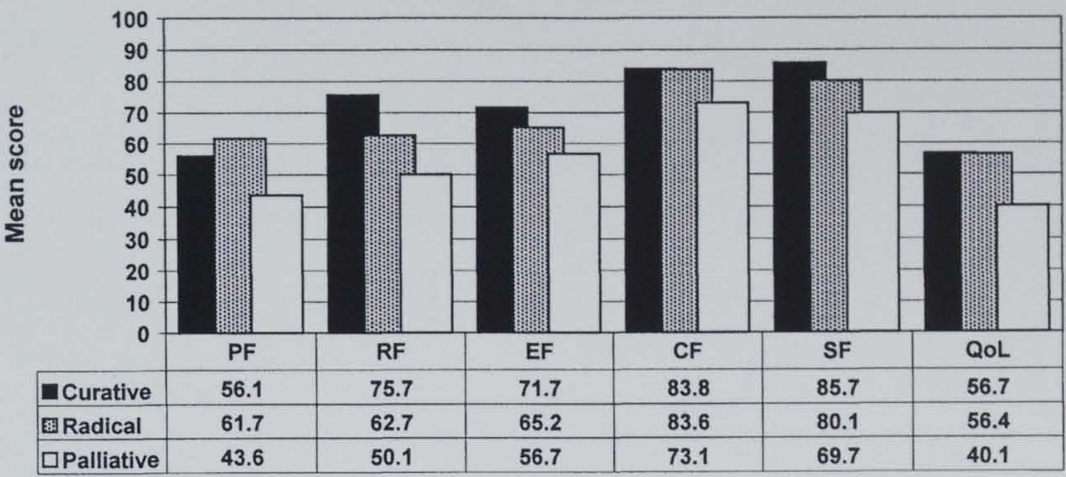

Prognostic groups and general symptom scales

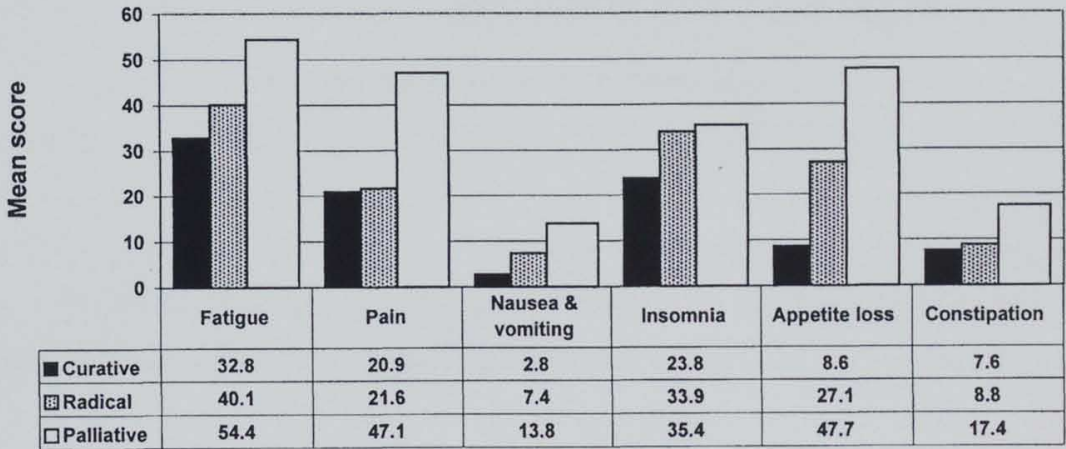

Prognostic groups and respiratory symptoms

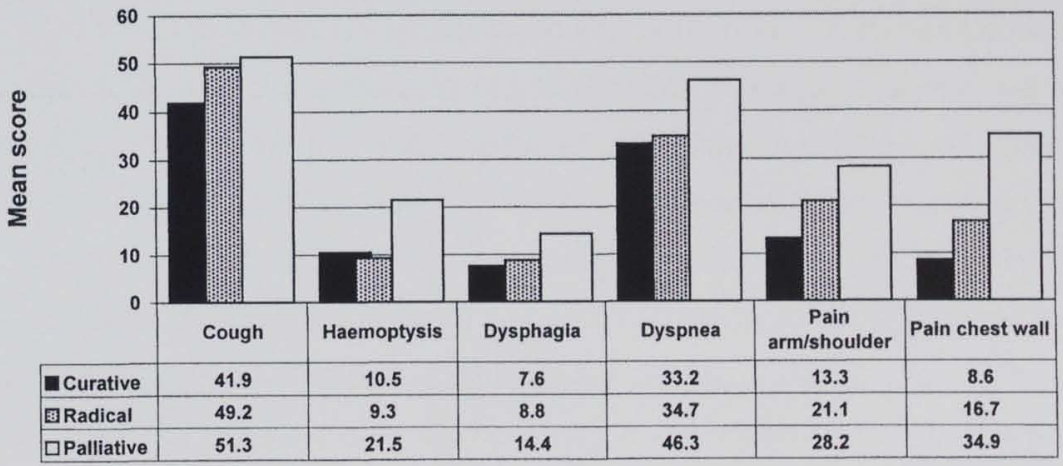


Multivariate analysis of the association between prognostic factors and QoL

In the first step of the GLM-MANOVA, the association between WHO performance status (WHO 0-1 versus WHO 2-3), weight loss (none versus $1-10 \%$ versus $>10 \%$ ) and different aspects of disease extension such as T-classification (T1-T2 versus T3-T4), nodal status ( $N 0$ versus $\mathrm{N}+$ ) and distant metastases (M0 versus $M 1$ ) and QoL was investigated. Age ( 0 70 versus $>70$ ) and gender were used as co-variates. In this analysis, a linear combination of the five functioning scales, the global QoL scale and the general symptoms fatigue and appetite loss assessed with the QLQ-C30 were used as the dependent variables. The results of this analysis are shown in Table 2.

WHO performance status, weight loss and age were associated significantly with the overall QoL outcome. Statistically significant differences between patients with WHO performance scores $0-1$ versus 2-3 were observed for all of the individual QLQ-C30 scales, with the exception of emotional functioning. However, the effect sizes were relatively small, varying from 0.02 for social functioning to 0.12 for physical functioning (Table 3 ).

A significant overall effect on QoL was also found for weight loss (Table 2). More detailed analysis showed that weight loss was associated significantly with patients' self-reported physical and social functioning, fatigue and appetite loss (Table 4). However, the effect sizes were very small, varying from 0.02 for social functioning to 0.11 for appetite loss. Age was also found to be related significantly to QoL, although this appeared to be restricted to self-reported physical functioning. Neither patients' gender nor TNM status was found to be related significantly to self-reported QoL (Table 2).

Table 2: Results of the GLM multivariate analysis for variance testing the effect of the classical prognostic factors on all EORTC QLQ-C30 scales.

\begin{tabular}{lcccc}
\hline Independent variables & Wilks' Lambda & F & Significance & $\begin{array}{c}\text { Effect size } \\
\text { (explained variance) }\end{array}$ \\
\hline T-classification & 0.954 & 1.46 & $\mathrm{~ns}$ & - \\
N-classification & 0.969 & 0.97 & $\mathrm{~ns}$ & - \\
M-classification & 0.968 & 0.99 & $\mathrm{~ns}$ & - \\
Weight loss & 0.830 & 2.97 & $<0.001$ & 0.09 \\
Performance status & 0.821 & 6.65 & $<0.001$ & 0.18 \\
Age & 0.901 & 3.36 & 0.001 & 0.10 \\
Sex & 0.972 & 0.87 & $\mathrm{~ns}$ & - \\
\hline
\end{tabular}


Chapter 3

Table 3: Results of the GLM multivariate analysis of variance testing the effect of WHO performance status on the individual EORTC QLQ-C30 scales.

\begin{tabular}{lcccc}
\hline Domain & \multicolumn{2}{c}{ Estimated mean scores } & $\begin{array}{c}\text { Pairwise } \\
\text { comparisons } \\
\text { (p-values) }\end{array}$ & $\begin{array}{c}\text { Effect size } \\
\text { (explained } \\
\text { variance) }\end{array}$ \\
\cline { 2 - 5 } WHO 0-1 & WHO 2-3 & & & \\
\hline Functioning scales & 62.1 & 38.2 & $<0.001$ & 0.12 \\
Physical functioning & 71.8 & 42.6 & $<0.001$ & 0.10 \\
Role functioning & 64.8 & 57.2 & $\mathrm{~ns}$ & - \\
Emotional functioning & 82.7 & 73.3 & 0.01 & 0.03 \\
Cognitive functioning & 83.5 & 74.8 & 0.04 & 0.02 \\
Social functioning & 53.6 & 41.1 & 0.001 & 0.04 \\
Global quality of life & & & & 0.03 \\
General symptoms & 39.2 & 50.3 & 0.01 & 0.04 \\
Fatigue & 23.1 & 39.3 & 0.001 & \\
Appetite loss & & & & \\
\hline
\end{tabular}

${ }^{a}$ The pairwise comparisons are based on the estimated mean scores in the multivariate analysis.

Table 4: Results of the GLM multivariate analysis of variance testing the effect of weight loss on the inidividual EORTC QLQ-C30 scales.

\begin{tabular}{|c|c|c|c|c|c|}
\hline \multirow[t]{2}{*}{ Domain } & \multicolumn{3}{|c|}{ Estimated mean scores } & \multirow{2}{*}{$\begin{array}{l}\text { Pairwise } \\
\text { comparison }^{\text {a }} \\
\text { (p-values) }\end{array}$} & \multirow{2}{*}{$\begin{array}{c}\text { Effect size } \\
\text { (explained } \\
\text { variance) }\end{array}$} \\
\hline & $\begin{array}{l}\text { No weight } \\
\text { loss }\end{array}$ & $\begin{array}{l}\text { Weight loss } \\
1-10 \%\end{array}$ & $\begin{array}{c}\text { Weight loss } \\
>10 \%\end{array}$ & & \\
\hline \multicolumn{6}{|l|}{ Functioning scales } \\
\hline Physical functioning & 55.4 & 52.5 & 42.6 & 0.02 & 0.03 \\
\hline Role functioning & 56.2 & 59.9 & 55.6 & ns & - \\
\hline Emotional functioning & 66.6 & 60.0 & 56.4 & ns & - \\
\hline Cognitive functioning & 80.2 & 76.2 & 77.6 & ns & - \\
\hline Social functioning & 83.6 & 81.3 & 72.5 & 0.05 & 0.02 \\
\hline Global quality of life & 51.0 & 48.9 & 42.1 & ns & - \\
\hline \multicolumn{6}{|l|}{ General symptoms } \\
\hline Fatigue & 33.9 & 44.7 & 55.7 & $<0.001$ & 0.08 \\
\hline Appetite loss & 14.7 & 35.4 & 43.9 & $<0.001$ & 0.11 \\
\hline
\end{tabular}

${ }^{\mathrm{a}}$ The pairwise comparisons are based on the estimated mean scores in the multivariate analysis. 


\section{Effect of respiratory symptoms on quality of life}

An additional multivariate analysis was performed to determine whether the presence and the severity of respiratory symptoms affected QoL. Of the six respiratory symptoms, only dyspnea was associated significantly with overall QoL (Table 5), with an effect being evident on physical, role, cognitive and social functioning, global quality of life and fatigue (table 6). The effect sizes ranged from 0.04 for role and social functioning to 0.20 for physical functioning.

Table 5: Results of the GLM multivariate analysis of variance testing the effect of respiratory symptoms on all EORTC QLQ-C30 scales.

\begin{tabular}{lcccc}
\hline Independent variables & Wilks' Lambda & F & Significance & $\begin{array}{c}\text { Effect size } \\
\text { (explained variance) }\end{array}$ \\
\hline Cough & 0.911 & 0.89 & ns & - \\
Heamoptysis & 0.879 & 1.24 & ns & - \\
Dyspnea & 0.645 & 4.44 & $<0.001$ & 0.14 \\
Dysphagia & 0.963 & 1.01 & $n s$ & - \\
Pain arm/shoulder & 0.860 & 1.46 & $n s$ & - \\
Pain chest wall & 0.871 & 1.33 & ns & - \\
\hline
\end{tabular}

Note: T-classification, N-classification, M-classification, WHO-performance, weight loss, age and sex were entered in the model as covariates.

Effect of changes in respiratory symptoms on general symptoms, functioning and global QoL

A multivariate analysis was performed to investigate the effect of changes of the respiratory symptoms assessed 6 weeks after the end of radiotherapy on general symptoms (i.e. fatigue and appetite loss), the physical and psychosocial functioning and global QoL.

A significant association between the change scores of the EORTC QoL scales and changes in dyspnea scores was found (table 7). No such association was observed between changes in the other

respiratory symptoms and self-reported functioning and QoL. A more detailed analysis showed that changes in dyspnea were associated significantly with changes in physical and role functioning, global QoL and fatigue (Table 8). However, the effect sizes were relatively small varying from 0.04 for role functioning to 0.09 for fatigue. 
Chapter 3

Table 6: Results of the GLM multivariate analysis of variance testing the effect of dyspnea on the individual EORTC QLQ-C30 scales.

\begin{tabular}{|c|c|c|c|c|c|c|}
\hline \multirow[t]{2}{*}{ Domain } & \multicolumn{4}{|c|}{ Estimated mean scores } & \multirow{2}{*}{$\begin{array}{c}\text { Comparisons } \\
\text { (p-values) }\end{array}$} & \multirow{2}{*}{$\begin{array}{l}\text { Effect size } \\
\text { (explained } \\
\text { variance) }\end{array}$} \\
\hline & Nil & Mild & Moderate & Severe & & \\
\hline \multicolumn{7}{|l|}{ Functioning scales } \\
\hline Physical functioning & 65.1 & 55.2 & 38.1 & 27.8 & $<0.001$ & 0.20 \\
\hline Role functioning & 76.5 & 69.6 & 57.0 & 54.6 & 0.02 & 0.04 \\
\hline Emotional functioning & 60.5 & 66.7 & 59.0 & 54.9 & ns & - \\
\hline Cognitive functioning & 81.2 & 88.0 & 77.9 & 69.9 & 0.001 & 0.07 \\
\hline Social functioning & 79.3 & 79.1 & 66.4 & 70.8 & 0.02 & 0.04 \\
\hline Global quality of life & 54.3 & 53.4 & 34.0 & 32.7 & $<0.001$ & 0.17 \\
\hline \multicolumn{7}{|l|}{ General symptoms } \\
\hline Fatigue & 26.8 & 33.9 & 52.0 & 60.1 & $<0.001$ & 0.18 \\
\hline Appetite loss & 30.4 & 28.6 & 32.2 & 35.8 & ns & - \\
\hline
\end{tabular}

Table 7: Results of the GLM multivariate analysis of variance testing the effect of changes in respiratory symptoms on all EORTC QLQ-C30 scales.

\begin{tabular}{lcccc}
\hline Independent variables & Wilks' Lambda & $F$ & Significance & $\begin{array}{c}\text { Effect size } \\
\text { (explained variance) }\end{array}$ \\
\hline Cough & 0.851 & 1.59 & ns & - \\
Heamoptysis & 0.889 & 1.14 & ns & - \\
Dyspnea & 0.827 & 1.88 & 0.02 & 0.09 \\
Dysphagia & 0.912 & 0.88 & ns & - \\
Pain arm/shoulder & 0.921 & 0.80 & ns & - \\
Pain chest wall & 0.867 & 1.39 & ns & - \\
\hline
\end{tabular}

\section{Discussion}

The primary question posed in the present study was whether pre-treatment QoL differed significantly between inoperable NSCLC with different prognostic profiles established on the basis of WHO performance status and a range of factors related to the extent of the disease.

Major differences were observed between these prognostic groups in survival. Although it is most likely that these differences in survival primarily reflect the clinical characteristics of the 
patients prior to treatment, it can not be ruled out that an increase in the total dose contributed to the survival benefit observed in the radical and curative groups as well ${ }^{9}$.

In the palliative treatment group, significantly lower levels of physical and psychosocial functioning and global QoL and more severe general and respiratory symptoms were observed as compared to the radical and curative groups. Only minor differences in functioning and symptom burden were observed between the radical and curative groups.

Prognostic group differences in self-reported QoL could be attributed to differences in WHO performance status, age and weight loss, and not to the TNM-classification. Although a marginal difference was found in WHO performance status between the radical and curative groups, the differences between these two groups were determined mainly by the stage of the tumour.

Table 8: Results of the GLM multivariate analysis of variance testing the effect of changes in dyspnea on the changes in individual EORTC QLQ-C30 scales.

\begin{tabular}{cccc} 
Domain & $\begin{array}{c}\text { Estimated mean change scores at } 6 \\
\text { weeks after radiotherapy }\end{array}$ & $\begin{array}{c}\text { Comparisons } \\
\text { (p-values) }\end{array}$ & $\begin{array}{c}\text { Effect size } \\
\text { (explained } \\
\text { variance) }\end{array}$ \\
\cline { 2 - 5 } & $\begin{array}{c}\text { Dyspnea } \\
\text { improved }\end{array}$ & $\begin{array}{c}\text { Dyspnea } \\
\text { unchanged }\end{array}$ & $\begin{array}{c}\text { Dyspnea } \\
\text { worse }\end{array}$
\end{tabular}

\begin{tabular}{|c|c|c|c|c|c|}
\hline \multicolumn{6}{|l|}{ Functioning scales } \\
\hline Physical functioning & 4.0 & -3.6 & -15.1 & 0.001 & 0.08 \\
\hline Role functioning & -5.6 & -3.8 & -24.2 & 0.04 & 0.04 \\
\hline Emotional functioning & 8.6 & 7.3 & 7.0 & ns & - \\
\hline Cognitive functioning & -1.8 & -0.2 & -5.6 & ns & - \\
\hline Social functioning & 0.1 & 0.1 & -8.3 & ns & - \\
\hline Global quality of life & 12.9 & 8.2 & 1.0 & 0.02 & 0.05 \\
\hline \multicolumn{6}{|l|}{ General symptoms } \\
\hline Fatigue & 0.7 & 5.7 & 19.3 & 0.001 & 0.09 \\
\hline Appetite loss & -2.4 & -9.2 & 1.5 & ns & - \\
\hline
\end{tabular}

Given the statistically significant association observed in this and other studies between WHO performance status and a number of the QoL scales ${ }^{1,10-12}$, one might be inclined to use performance status as a proxy measure for QoL. However, this remains controversial because of the lack of responsiveness of performance status scores to worsening toxicity ${ }^{13}$, 


\section{Chapter 3}

the inconsistent ratings of the same patient by different evaluators ${ }^{14,15}$ and the poor correlation with symptom prevalence ${ }^{16}$. Furthermore, although in the present study WHO performance status was the most important determinant in distinguishing different levels of QoL, the total amount of variance in various QoL scores accounted for by performance status was rather low, in particular for emotional, cognitive and social functioning.

The relationship between prognostic factors and baseline QoL has been investigated by several other studies. Curran et al. reported on a study in which baseline QoL was investigated in patients with advanced prostate cancer ${ }^{17}$. The prognostic factors investigated included disease stage, pain caused by the primary tumour or bony metastases, WHO performance status and hormone resistance. Significantly more pain, worse physical and role functioning and worse overall QoL was found in the patients with poor versus good prognostic features. Ringdal et al. also investigated the relationship between QoL in different prognostic groups, i.e. curative, palliative treatment to delay tumour progression, or palliative treatment of symptoms ${ }^{7}$. This classification into prognostic groups is comparable to that employed in the present study. Although they found significant differences between the prognostic groups on a number of scales of the EORTC QLQ-C30, in contrast to the results of the present study, no such differences were observed in psychological or social functioning. These apparently conflicting results might be explained by differences in diagnosis and subsequent prognosis. In the study of Ringdal et al, various sites of malignant diseases were included and the observed survival varied from $5 \%$ after 200 months for the poor prognostic group to $80 \%$ after 150 months for the good prognostic group. These survival rates were much better than those found in the present study. In both studies, patients were fully informed about their diagnosis, prognosis and aims of treatment at the time of assessment of baseline QoL. These results suggest that psychological functioning may be affected primarily when the patients' prognosis is relatively poor.

One of the aims of the current study was to investigate the effect of respiratory symptoms on QoL. Results of the multivariate analysis indicated that dyspnea was the most important respiratory symptom affecting all EORTC scales, with the exception of emotional functioning. The aetiology of dyspnea is multi-factorial. In this category of patients, it may be the result of pleural effusion, atelectasis due to bronchial obstruction by the primary tumour or enlarged mediastinal lymph nodes, pain in the chest wall by direct tumour extension, as well as of co-morbidity. Subsequently, the question arose as to whether palliation of dyspnea by radiotherapy resulted in improvement of $\mathrm{QoL}$ as well. The results showed that 
there was a significant association between improvement of dyspnea and changes in physical and role functioning, global QoL and fatigue. For patients who experienced worsening of dyspnea, the change scores for physical and role functioning were -15.1 and -24.2 respectively. The mean change score for global QoL increased 12.9 points among patients who experienced improvement of dyspnea. The question arises how the magnitude of these changes should be interpreted. Osoba and co-workers investigated the significance of changes in health-related QoL scores assessed with the QLQ-C30 by means of a subjective significance questionnaire (SSQ), in which patients were posed direct questions about perceived changes in physical, emotional and social functioning and in global QoL ${ }^{18}$. For patients who indicated "no change" in the SSQ, the mean scores of the corresponding QLQ-C30 domains was not significantly different from 0 . For patients who indicated "moderate" change either for better or worse, the mean change in scores was about 10 to 20 points on a 100-point scale, and for "very much" change, greater than 20 points. These results indicate that changes in dyspnea may have a significant effect on these functioning scales and global QoL. The results showed that palliation of other respiratory symptoms is not necessarily associated with improvement of general symptoms, physical and psychological functioning or global QoL.

In conclusion, in inoperable NSCLC, classical prognostic factors including performance status and weight loss are associated significantly with self-reported QoL. However, changes in performance status account only partly for the variance observed in different QoL scales, indicating that performance status cannot be used as a proxy measure for QoL. Although generally assumed, no association could be found between TNM-classification and QoL. Dyspnea is associated significantly with patients' functioning, fatigue and global QoL at baseline and palliation of this symptom may have a beneficial effect on these health outcomes. 


\section{$\square$ References}

1. Aaronson NK, Ahmedzai S, Bergman B, et al. The European Organization for Research and Treatment of Cancer QLQ-C30: a quality of life instrument for use in international clinical trials in oncology. J Natl Cancer Inst 1993; 85: 365-376.

2. McCarthy DM. Quality of life: A critical assessment. Scan J Gastroenterol 1995; 30: 141-146.

3. Cella DF, Tulsky DS, Gray G, et al. The functional Assessment of Cancer Therapy Scale: Development and validation of the general measure. J Clin Oncol 1993; 11: 570-579.

4. Osoba D, Zee B, Wan D, et al. Psychometric properties and responsiveness of the EORTC quality of life questionnaire (QLQ-C3O) in patients with breast, ovarian and lung cancer. Qual Life Res 1994; 3: 143-154.

5. Aaronson NK, Ahmedzai $S$, Bullinger $M$, et al. The EORTC core quality of life questionnaire: interim results of an international field study. In: Osoba D (ed.) Effect of Cancer on Quality of Life Boston; CRC Press, 1991: $185-204$.

6. Cella DF, Orofiamma B, Holland JC, et al. The relationship of psychological distress, extent of disease, and performance status in patients with lung cancer. Cancer 1987; 60: 1661-1667.

7. Ringdal GI, Ringdal K, Kvinnsland S, Gotestam KG. Quality of life of cancer patients with different prognoses. Qual Life Res 1994; 3: 143-154.

8. Bergman B, Aaronson, NK, Ahmedzai S, et al. The EORTC QLQ-LC13: a modular supplement to the EORTC Core Quality of Life Questionnaire (QLQ-C30) for use in lung cancer clinical trials in oncology. Eur J Cancer 1994; 30A: 635-642.

9. Perez CA, Stanley K, Grundy G, et al. Impact of irradiation technique and tumor extent in tumor control of patients with unresectable non-oat cell carcinoma of the lung. Cancer 1982; 50: 1091-1099.

10. Nou $E$ and Aberg T. Quality of life in patients with surgically treated bronchial carcinoma. Thorax 1980; 35: 255-263.

11. Eguchi K, Fukutani $M$, Kanazawa $M$, et al. Feasibility study on quality of life questionnaire for patients with advanced lung cancer. Jpn J Clin Oncol 1992; 22: 185-193.

12. Buccheri GF, Ferrigno D, Tamburini $M$, et al. The patient's perception of his own quality of life might have adjunctive prognostic significance in lung cancer. Lung Cancer 1995; 12: 45-58.

13. Geddes DM, Dones L, Hill E, et al. Quality of life during chemotherapy for small cell lung cancer: assessment and use of daily diary card in a randomized trial. Eur J Cancer 1990; 26: 484-492.

14. Hutchinson TA, Boyd NF and Feinstein AR. Scientific problems in clinical scales as demonstrated in the Karnofksy index of performance status. J Chron Dis 1979; 32: 661-666.

15. Oken $\mathrm{MM}, \mathrm{Creech} \mathrm{RH}$, Tormey $\mathrm{DC}$, et al. Toxicity and response criteria of the Eastern Cooperative Oncology Group. Am J Clin Oncol 1982; 5: 649-655.

16. Mor V, Laliberte L, Morris JN and Wiemann M. The Karnofsky performance status scale: an examination of its reliability and validity in a research setting. Cancer 1984; 53: 2002-2007.

17. Curran D, Fossa $\mathrm{S}$, Aaronson $\mathrm{N}$, et al. Baseline quality of life of patients with advanced prostate cancer. Eur $\mathrm{J}$ Cancer 1997; 33 (11): 1809-1814.

18. Osoba, D. Rodrigues, G. Myles, J, et al. Interpreting the significance of changes in quality of life scores. J Clin Oncol 1998; 16: 139-144. 


\title{
Chapter 4
}

\section{The prognostic impact of quality of life assessed with the EORTC QLQ-C30 in inoperable non-small cell lung carcinoma treated with radiotherapy}

\author{
Hans Langendijk \\ Neil Aaronson \\ Jos de Jong \\ Guul ten Velde \\ Martin Muller \\ Miel Wouters
}

Published in

Radiotherapy and Oncology 2000; 55: 19-25 


\section{Abstract}

Purpose: The purpose of this study was to assess the prognostic significance of pretreatment quality of life (QoL) scores and symptom scores in a group of patients treated with high dose radiotherapy.

Material and methods: A total of 198 patients treated with external irradiation ( $\geq 60 \mathrm{~Gy}$ ) were included. In all these patients, baseline QoL was assessed using the EORTC QLQC30. The prognostic significance of a number of non-QoL and QoL parameters with regard to survival was estimated in both univariate and multivariate analyses.

Results: In a multivariate model including the non-QoL parameters, performance status, weight loss and $\mathrm{N}$-classification were independent prognostic factors for survival. After entering the QoL parameters in the model, global QoL was the strongest prognostic factor, while performance status lost its significance. Subsequently, a significant interaction term was found between $\mathrm{N}$-classification and global QoL, indicating that global QoL was an independent prognostic factor but that the effect varied as a function of $\mathrm{N}$-status. In $\mathrm{N}+$ patients, the median survival in the group with low scores for global QoL was 4.5 months, which was significantly worse $(p<0.0001)$ compared to the high score group in which the median survival was 12.9 months.

Conclusion: Global QoL is a strong prognostic factor for survival in patients with NSCLC who have pathological lymph nodes at presentation and who are treated with radical or curative radiotherapy. 


\section{Introduction}

Nowadays, a range of instruments is currently available which allow reliable and valid measurement of several aspects of quality of life (QoL) ${ }^{1-6}$. Typically, patient-rated QoL has been used as an outcome parameter to compare different treatment approaches in clinical trials ${ }^{7-10}$. A number of studies also reported on the correlation between several aspects of QoL and subsequent survival ${ }^{11-18}$. In inoperable non-small cell lung carcinoma (NSCLC), performance status ${ }^{19-27}$, weight loss ${ }^{20,22,26-29}$ and stage of disease ${ }^{21,23-29}$ are the most important and well-accepted prognostic factors. These factors are commonly used to select patients for different treatment approaches. Prognostic associations between QoL scores and survival duration have been reported for metastatic lung cancer ${ }^{13}$, for inoperable NSCLC treated with chemotherapy and/or low dose radiotherapy ${ }^{12}$ and for other primary sites ${ }^{11,14,15}$. However, no such data are available with regard to the prognostic significance of QoL scores in patients with inoperable NSCLC who are regarded to have a relatively good prognosis and therefore are candidates for radical radiotherapy.

The identification of prognostic factors in patients with inoperable non-small cell lung cancer (NSCLC) is important because they may provide insight into the disease process, providing direction for further studies, and may be important in defining the eligibility criteria for new clinical trials and stratifying patients by risk groups. Furthermore, prognostic factors are important when therapeutic options depend on pre-treatment characteristics of the patients.

The primary objective of the current study was to assess the prognostic significance of pre-treatment QoL scores and symptom scores, assessed with the EORTC QLQ-C30 in a group of patients treated with high dose radiotherapy. The hypothesis to be tested was that low scores for baseline QoL and high scores for general symptoms would be associated with worse survival. The secondary aim of this study was to identify a subset of patients with poor outcome despite high dose radiotherapy that would possibly benefit more from a shorter course of palliative radiotherapy. 


\section{a Material and methods}

\section{Patients}

Patients eligible for the study were those with stage I, II, IIla or IIIb according to the UICC 1992 staging system ${ }^{30}$, primarily treated with radical or curative radiotherapy (at least 60 Gy) and a WHO performance status ranging from 0 to 2. Patients with supraclavicular lymph node metastases, previously treated patients or patients treated with external irradiation in combination with endobronchial brachytherapy were excluded from the study. Patients were included in a prospective, non-randomised study in which changes in QoL and general and respiratory symptoms were assessed before and after radiotherapy. Therefore, baseline (i.e., pre-treatment) QoL scores were available for all patients. From March 1994 to November 1996, 198 patients met the eligibility criteria and completed baseline QoL questionnaires. The characteristics of these patients are presented in Table 1.

The staging procedure consisted of a physical examination, chest radiography, bronchoscopy and computed tomography of the chest (CT-chest) including the liver and adrenal glands. Bone scintigraphy was performed in 173 cases $(75 \%)$. In the remaining patients, there were no indications for bony metastases. In 52 patients (23\%), mediastinoscopy was performed and positive nodes were found. Patients were also classified as having mediastinal lymph node metastases in case enlarged lymph nodes on the CT-chest. A node was considered positive in case the smallest diameter was $\geq 1$ centimetre. Patients were considered inoperable because of advanced loco-regional tumour extension in 146 patients (74\%), poor lung function in 87 patients (44\%), comorbidity in 26 patients $(13 \%)$ and advanced age in 6 patients $(3 \%)$. In some patients more than one reason was present.

\section{Radiotherapy protocol}

Patients with stage I or II disease (UICC 1992) stage Illa and stage Illb disease (UICC 1992) without supraclavicular lymph node metastases and a WHO-performance $\leq 2$ were selected for the radical radiotherapy schedule. The target volume of the initial field encompassed the primary tumour and enlarged lymph nodes with a margin of 2 centimetres, the ipsilateral hilus and the mediastinal lymph nodes on both sides. The 
Table 1: Patients characteristics

\begin{tabular}{|c|c|c|c|}
\hline Variable & & Number & $\%$ \\
\hline \multirow[t]{4}{*}{ T-classification } & T1 & 29 & $15 \%$ \\
\hline & $\mathrm{T} 2$ & 77 & $39 \%$ \\
\hline & T3 & 28 & $14 \%$ \\
\hline & T4 & 64 & $32 \%$ \\
\hline \multirow[t]{4}{*}{ N-classification } & No & 88 & $44 \%$ \\
\hline & $\mathrm{N} 1$ & 3 & $2 \%$ \\
\hline & N2 & 85 & $43 \%$ \\
\hline & N3 & 22 & $11 \%$ \\
\hline \multirow[t]{4}{*}{ Stage (UICC 1992) } & Stage I & 53 & $27 \%$ \\
\hline & Stage II & 2 & $1 \%$ \\
\hline & Stage IIla & 65 & $33 \%$ \\
\hline & Stage IIIb & 78 & $39 \%$ \\
\hline \multirow[t]{3}{*}{ Tumour type } & Squamous cell carcinoma & 118 & $60 \%$ \\
\hline & Adenocarcinoma & 30 & $15 \%$ \\
\hline & Undifferentiated carcinoma & 50 & $25 \%$ \\
\hline \multirow[t]{2}{*}{ Sex } & Male & 168 & $85 \%$ \\
\hline & Female & 30 & $15 \%$ \\
\hline \multirow[t]{2}{*}{ Age } & $0-70$ years & 101 & $51 \%$ \\
\hline & Range & 97 & $49 \%$ \\
\hline \multirow[t]{3}{*}{ WHO-performance } & WHOO & 82 & $41 \%$ \\
\hline & WHO 1 & 91 & $46 \%$ \\
\hline & WHO 2 & 25 & $13 \%$ \\
\hline \multirow[t]{3}{*}{ Weight loss } & $0-10 \%$ & 162 & $83 \%$ \\
\hline & $>10 \%$ & 34 & $17 \%$ \\
\hline & unknown & 2 & \\
\hline
\end{tabular}

target volume of the initial field was treated with fraction doses of $2.25 \mathrm{~Gy}$ (4 times per week) to a total dose of $45 \mathrm{~Gy}$.

In case of stage I or II disease (UICC 1992), the total dose depended on the maximum diameter of the tumour. In case of a tumour diameter $\geq 4$ centimetres the total dose to the primary site was $60 \mathrm{~Gy}$, according to the schedule as described for stage IIla and IIlb.

In case of stage I or II disease with a maximum diameter of the primary site of $<4$ centimetres and a WHO-performance $\leq 2$, the curative schedule was selected. The target volume for the initial field was similar to those of the radical schedule. However, in these 
patients the dose per fraction was $2 \mathrm{~Gy}$ ( 5 times per week). The initial field was treated up to a total dose of 46 Gy followed by a boost up to the primary site with a 1 centimetre margin to a total dose of $70 \mathrm{~Gy}$. In some patients with poor lung function and peripherally located tumours (T1NO), the target volume of the initial field only included the primary tumour with a margin of 2 centimetre.

Field arrangements were assessed by a localisation/simulation procedure or by CT-assisted planning and individual blocks were used to shield normal tissue. In general, the initial field was treated using two opposing anterior-posterior/posterior-anterior fields. For the boost, a three- or four-field technique was used in most cases. The dose was specified in the isocentre and the target volumes described received at least $90 \%$ of the prescribed dose. In all cases correction was made for lung tissue density (0.3).

\section{Quality of life assessment}

For the assessment of QoL, the EORTC QLQ-C30 questionnaire (version 1.0) was used ${ }^{5}$. This questionnaire contains 30 questions addressing various aspects of QoL. For the analysis of the present study, the association between survival and the five functional scales (physical, role, cognitive, emotional and social), the global health/quality of life scale (global QoL) and a number of symptom scales (fatigue, appetite loss, pain and dyspnea) was investigated. Following EORTC guidelines, sub scale scores were linearly converted to a 0 to 100 scale. For the functional and global health status/quality of life scales, higher scores represent a better level of functioning. For the symptom scales higher scores represent greater symptom burden. The baseline questionnaires were administered approximately 1 week before the start of radiotherapy. The reliability and validity of the QLQ-C30 have been confirmed in a number of international studies of patients with different primary tumour sites $5,31,32$

\section{Statistics}

Survival was determined from the first day of radiotherapy. Patients alive at the last day of follow up were censored. A multivariate analysis using the Cox proportional hazards model was performed to identify covariates that were significantly associated with survival ${ }^{33}$. For the purpose of this study, explanatory variables were selected using a stepwise forward procedure with an enter limit fixed at the 0.05 level. 
Survival curves were estimated with the Kaplan-Meier method ${ }^{34}$. To test the statistical significance of differences between curves, the log rank test was used ${ }^{35}$. The statistical analysis was performed using the SPSS/PC software package.

\section{口 Results}

\section{Non-QoL prognostic factors}

To identify non-QoL prognostic factors, a multivariate analysis was performed (the non-QoL model). The following potential prognostic variables were dichotomised and included in the regression model: T-classification (T1-T2 vs T3-T4), N-classification (NO vs N+), WHO performance status (WHO 0 vs WHO 1-2), weight loss $(0-10 \%$ vs $>10 \%)$, histologic tumour type (squamous cell vs non-squamous cell carcinoma), sex (male vs female) and age (0-70 vs $>70$ years). Following a forward stepwise procedure, $\mathrm{N}$-classification, weight loss and WHO performance status were found to be associated significantly with survival (table 2a). A borderline significance was found for T-classification $(p=0.06)$. No association was found between survival and other non-QoL variables and no significant interaction terms were detected.

Table 2: Results of the Cox regression analysis

\begin{tabular}{|c|c|c|c|c|c|c|}
\hline Variable & Score & Coefficient & SE & RR & RR: $95 \%-c i$ & p-value \\
\hline \multicolumn{7}{|c|}{ a. Only non-QoL variables entered } \\
\hline N-classification & $N O=0 ; N+=1$ & 0.49 & 0.16 & 1.6 & $1.2-2.2$ & 0.002 \\
\hline WHO performance status & WHO O $=0 ; W H O>0=$ & 10.38 & 0.16 & 1.5 & $1.0-2.0$ & 0.02 \\
\hline Weight loss & $0-10 \%=0 ;>10 \%=1$ & 0.43 & 0.21 & 1.5 & $1.0-2.3$ & 0.04 \\
\hline \multicolumn{7}{|c|}{ b. QoL scores entered as continuous variables } \\
\hline N-classification & $N O=0 ; N+=1$ & 1.86 & 0.42 & 6.4 & $2.8-14.6$ & $<0.0001$ \\
\hline Global quality of life & & -0.0218 & 0.0051 & 0.9784 & $0.9687-0.9882$ & $<0.0001$ \\
\hline Weight loss & $0-10 \%=0 ;>10 \%=1$ & 0.58 & 0.20 & 1.8 & $1.2-2.7$ & 0.004 \\
\hline N-classification*Global QoL & & 0.02 & 0.007 & & & 0.002 \\
\hline WHO performance status & $W H O O=0 ; W H O>0=$ & 10.26 & 0.16 & & & 0.12 \\
\hline \multicolumn{7}{|c|}{ c. QoL scores entered as dichotomised variables } \\
\hline Global quality of life & $0=$ low, $1=$ high & -0.52 & 0.16 & 1.7 & $1.2-2.3$ & 0.001 \\
\hline $\mathrm{N}$-classification & $N O=0 ; N+=1$ & 0.60 & 0.16 & 1.8 & $1.3-2.5$ & 0.002 \\
\hline Weight loss & $0-10 \%=0 ;>10 \%=1$ & 0.50 & 0.20 & 1.7 & $1.1-2.5$ & 0.01 \\
\hline N-classification*Global QoL & & 0.9 & 0.31 & & & 0.004 \\
\hline WHO performance status & $W H O O=0 ; W H O>0=$ & $=10.27$ & 0.16 & & & 0.09 \\
\hline
\end{tabular}




\section{Prognostic value of QoL}

In the univariate analysis, a significant association between global QoL and survival was observed (table 3). The negative coefficient indicates that higher scores for QoL are associated with better survival. For role functioning, a borderline significance was found. Fatigue and appetite loss were also significantly associated with survival. The positive coefficient indicates that lower scores for these symptoms are associated with better survival. No association was observed between the remaining functioning and symptom scales and survival.

Table 3: Results of the univariate analyses of QoL scores (continuous variables)

\begin{tabular}{lcccccc}
\hline Variable & Number & Coefficient & SE & Hazard & $95 \%$-ci & p-value \\
\hline Physical functioning & 198 & -0.0045 & 0.0029 & 0.9955 & $(0.9898-1.0012)$ & 0.12 \\
Role functioning & 198 & -0.0036 & 0.0021 & 0.9964 & $(0.9923-1.0005)$ & 0.08 \\
Emotional functioning & 198 & -0.0038 & 0.0027 & 0.9962 & $(0.9910-1.0015)$ & 0.16 \\
Cognitive functioning & 198 & -0.0028 & 0.0036 & 0.9972 & $(0.9902-1.0043)$ & 0.44 \\
Social functioning & 198 & -0.0034 & 0.0030 & 0.9966 & $(0.9907-1.0026)$ & 0.26 \\
Global quality of life & 198 & -0.0069 & 0.0030 & 0.9932 & $(0.9873-0.9991)$ & 0.02 \\
Fatigue & 198 & 0.0065 & 0.0026 & 1.0065 & $(1.0014-1.0116)$ & 0.01 \\
Pain & 198 & 0.0025 & 0.0027 & 1.0025 & $(0.9973-1.0078)$ & 0.35 \\
Dyspnea & 196 & 0.0049 & 0.0028 & 1.0050 & $(0.9994-1.0105)$ & 0.08 \\
Appetite loss & 198 & 0.0069 & 0.0023 & 10.069 & $(1.0024-1.00114)$ & 0.002 \\
\hline
\end{tabular}

Subsequently, a multivariate analysis was performed to investigate the association between the QoL scales and survival, with adjustment for the non-QoL prognostic factors associated significantly with survival, i.e. N-classification, weight loss and WHO performance status (the QoL model). When the various QoL scales were entered as continuous variables, in this analysis only global QoL was found to be associated significantly with survival (table $2 b$ ). In this model, $\mathrm{N}$-classification and weight loss remained significant prognostic factors while WHO performance status exhibited no longer significant value. A borderline significance was found for appetite loss $(p=0.07)$. The inclusion of other QoL scales in the model did not provide additional independent prognostic information. As a continuous variable is more likely to prove significant than a dichotomised variable, the multivariate analysis was repeated with the QoL scales entered as dichotomised variables. All scales were divided at 
the median. Again, global QoL, N-classification and weight loss were independent prognostic factors (Table $2 \mathrm{c}$ ).

In the QoL model, a significant interaction term was found between $\mathrm{N}$-classification and global QoL, indicating that global QoL was an independent prognostic factor, but that the effect depended on $\mathrm{N}$-status. To confirm this interaction, a univariate analysis was performed to investigate the prognostic value of global $\mathrm{QoL}$ after stratification for $\mathrm{N}$-status. For this purpose, the study sample was divided into two groups: a group of patients with a mean score for global QoL greater than or equal to the median value (high QoL group) and a group with a mean score less than the median value (low QoL group). In N0 patients, the median survival in the low QoL group was 11.5 months (95\%-ci: $5.8-17.3$ ) compared to 13.8 months (95\%-ci: $10.9-16.8$ ) in the high QoL group ( $p=0.71$ ). However, in $\mathrm{N}+$ patients, the median survival in the low QoL group was 4.5 months (95\%-ci: $2.4-6.6$ ), which was significantly worse $(p<0.0001)$ as compared to the high QoL group in which the median survival was 12.9 months (95\%-ci: $9.2-16.6$ ) (Figure 1). In the multivariate analysis, no other significant interaction terms could be detected.

A statistically significant but modest correlation was observed between global QoL and performance status (Pearson correlation: $-0.25, p<0.001$ ).

\section{Identification of prognostic groups}

To investigate which model (the non-QoL model or the QoL-model) was most appropriate for identifying a subgroup of patients with different survival outcomes; we calculated the number of risk factors present for each patient. In particular, we were interested in identifying a subset of patients with such poor survival that short course palliative radiotherapy might be considered as more appropriate.

In the non-QoL model, a subset of patients with 3 poor prognostic factors $(\mathrm{N}+$, weight loss > $10 \%$ and $P S>0$ ) could be identified, with a median survival of 2.8 months (95\%-ci: $0.0-5.8$ months) and no patients alive after 7.8 months (Table 4a)(Figure 2). This was the case in 12 out of 196 patients $(6 \%)$.

In the QoL model, a subset of patients with 3 poor prognostic factors $(\mathrm{N}+$, weight loss > $10 \%$ and global QoL < median) could be identified as well. In this subset of patients, the median survival was 2.8 months and no survivors were observed after 6.8 months (Table $4 \mathrm{~b})$ (Figure 3). This was the case in 12 out of 108 patients (11\%). 
Figure 1: Survival by global QoL stratified by N-classification.

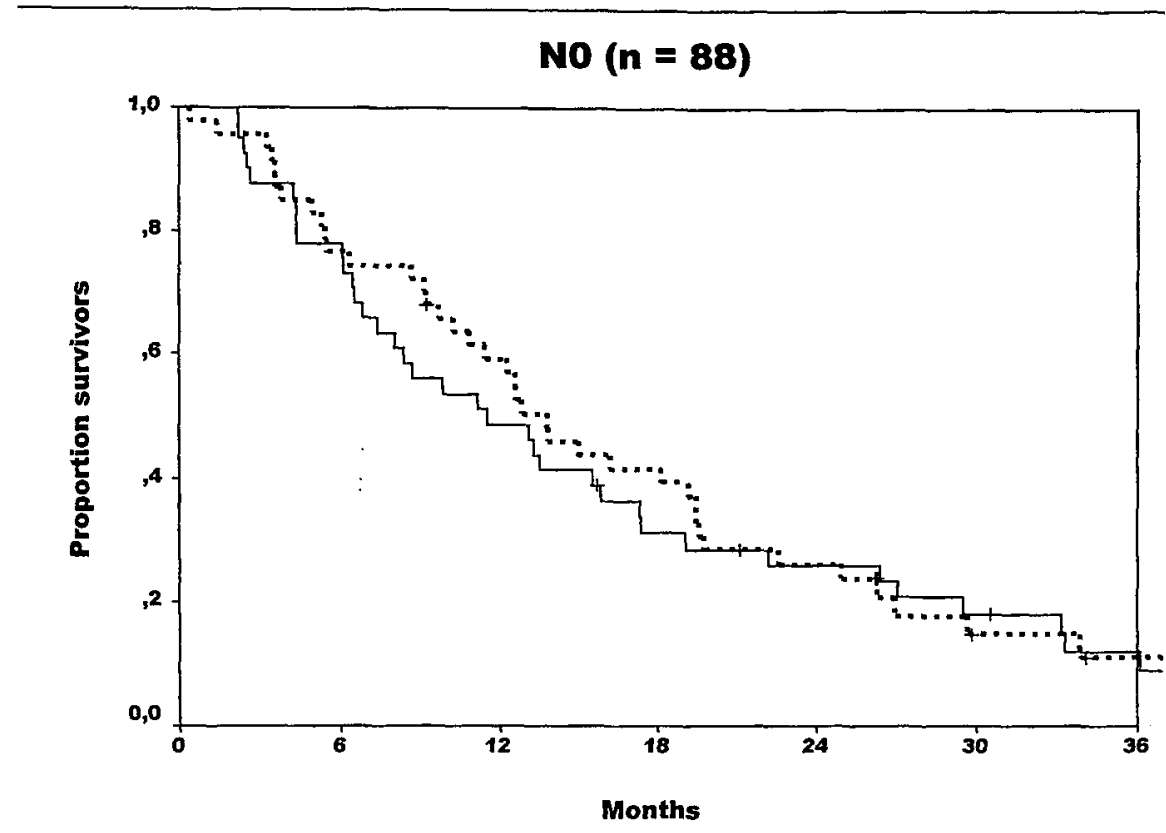

N1-N3 (n = 110)

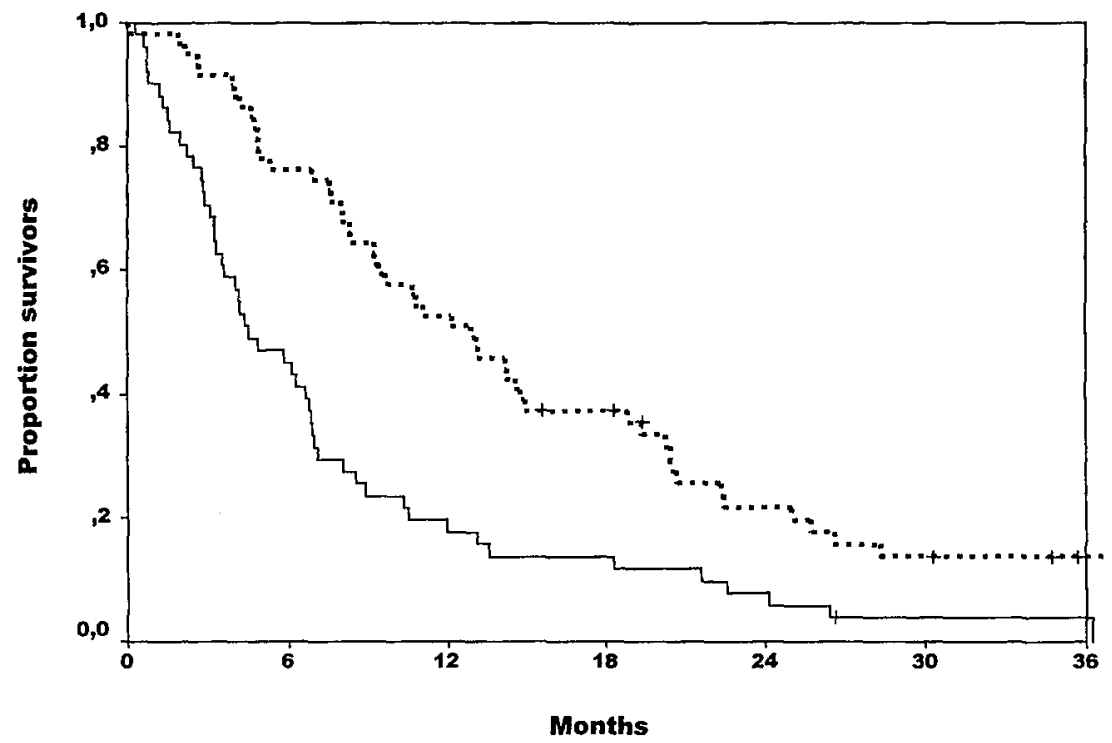

Straight line $=$ low global QoL score, dotted line = high global QoL score. 
Figure 2: Estimated survival curves for patients with different numbers of non-QoL independent risk factors based on the Cox model.

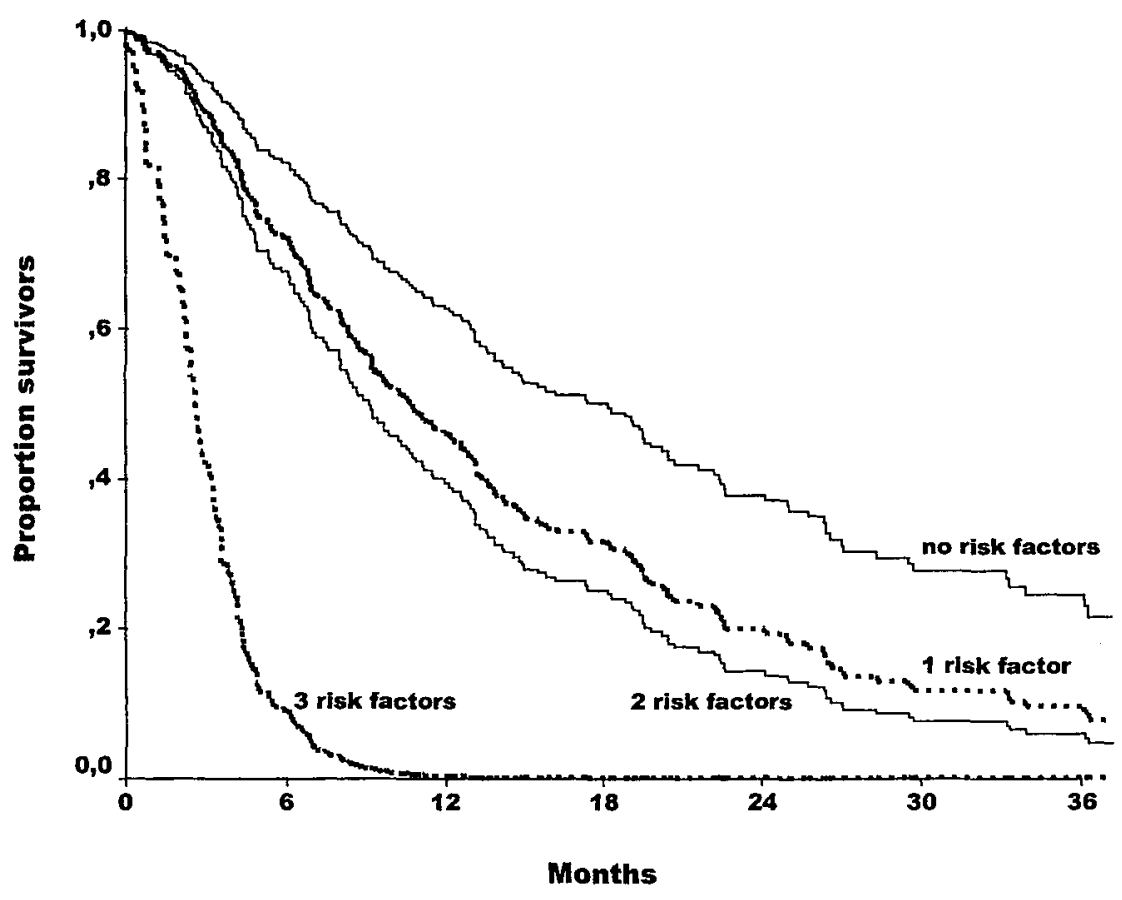

Independent risk factors are N-clasification, PS, and weight loss.

Table 4: Results of the Cox regression model including the non-QoL model and the QoL model

\begin{tabular}{|c|c|c|c|c|}
\hline Number of risk factors & Number & Median survival & $\operatorname{RR}(95 \%-c i)^{\star}$ & P-value* \\
\hline \multicolumn{5}{|c|}{ a. All patients (prognostic factors: $N$-classification, weight loss and $P S$ ) } \\
\hline 0 risk factors & 28 & 16.2 & 1.0 & \\
\hline 1 risk factor & 92 & 10.9 & $1.7(1.0-2.7)$ & 0.04 \\
\hline 2 risk factors & 64 & 8.3 & $2.0(1.2-3.3)$ & 0.07 \\
\hline 3 risk factors & 12 & 2.8 & $12.4(5.8-26.6)$ & $<0.0001$ \\
\hline \multicolumn{5}{|c|}{ b. N+ patients (prognostic factors: weight loss and global QoL) } \\
\hline $\mathrm{N}+$ but no other risk factors & 54 & 12.2 & $1.6(0.9-2.7)$ & 0.13 \\
\hline $\mathrm{N}+$ and 1 other risk factor & 42 & 6.7 & $3.1(1.7-5.6)$ & 0.0001 \\
\hline $\mathrm{N}+$ and 2 other risk factors & 12 & 2.8 & $13.2(5.8-29.9)$ & $<0.0001$ \\
\hline
\end{tabular}

"RR and p-value compared to NO, weight loss $<10 \%$ and $P S O$ or high global QOL score 


\section{口 Discussion}

In the present study, the prognostic significance of patient-rated QoL assessed with the QLQ-C30 was investigated in a group of patients with inoperable NSCLC selected for high dose radiotherapy. When only the non-QoL variables were taken into account, $N$ classification, weight loss and performance status were found to be independent prognostic factors. These variables are well known prognostic indicators in NSCLC. When the scores for several QoL domains were entered in the regression model, the pre-treatment score for global QoL emerged as the strongest independent prognostic factor, even after allowance for the non-QoL prognostic indicators. Interestingly, after entering the QoL variables into the regression model, performance status lost its prognostic significance. Ganz and co-workers found comparable results in NSCLC. In their study, the Functional Living Index-Cancer (FLIC) was used to assess QoL in 40 patients with stage IV metastatic NSCLC treated with supportive care, including palliative radiotherapy, psychosocial support, analgetics and nutritional support with or without combination chemotherapy ${ }^{13}$. In this study, a significant association between the FLIC-score, marital status, and survival was found. Furthermore, as in the present study, performance status was no longer a prognostic indicator for survival. Kaasa and co-workers reported on the prognostic significance of QOL in a group of patients with stage II and III NSCLC treated with a combination of chemotherapy and radiotherapy or radiotherapy alone. In a multivariate analysis patients' self-reported general symptoms and psychosocial well being were the best predictors of survival while initial performance status, weight loss and stage of disease did not show significant prognostic value ${ }^{12}$. Other investigators have reported similar results in advanced breast cancer ${ }^{14}$. As the prognostic significance of performance status in inoperable NSCLC has been confirmed in a considerable number of studies ${ }^{19-27}$, we were somewhat surprised by these findings.

Our results and those of others suggest that patients' self-rated QoL has greater predictive power than performance status as assessed by the physician. It is well known that current health status is predictive of future morbidity and mortality. In QoL assessment, patients are simply asked to rate their current health and health-related QoL. They seem to do this pretty well.

In view of these results, one might argue for abandoning performance status as a prognostic factor in favour of patient's self-reported QoL. However, such a recommendation may be premature for a number of reasons. First, it has to be noted that, in the present 
analysis, only patients with a WHO performance status equal or less than 2 were included. This limited range could account for the relatively week predictive power observed. In the other studies concerning patients with NSCLC mentioned above, the range in performance status was also limited ${ }^{12-13}$. Secondly, in the present study, global QoL was only found to have prognostic significance in the subset of patients with a relatively poor prognosis, namely in those with pathological lymph nodes and not in those with NO disease. On the other hand, the fact that QoL emerges as an important prognostic factor in a number of studies, despite the different ways in which QoL has been defined and operationalised, provides strong evidence for the robustness of the QoL-survival association

Figure 3: estimated survival curves for $\mathrm{N}+$ patients with different numbers of independent risk factors based on the Cox model.

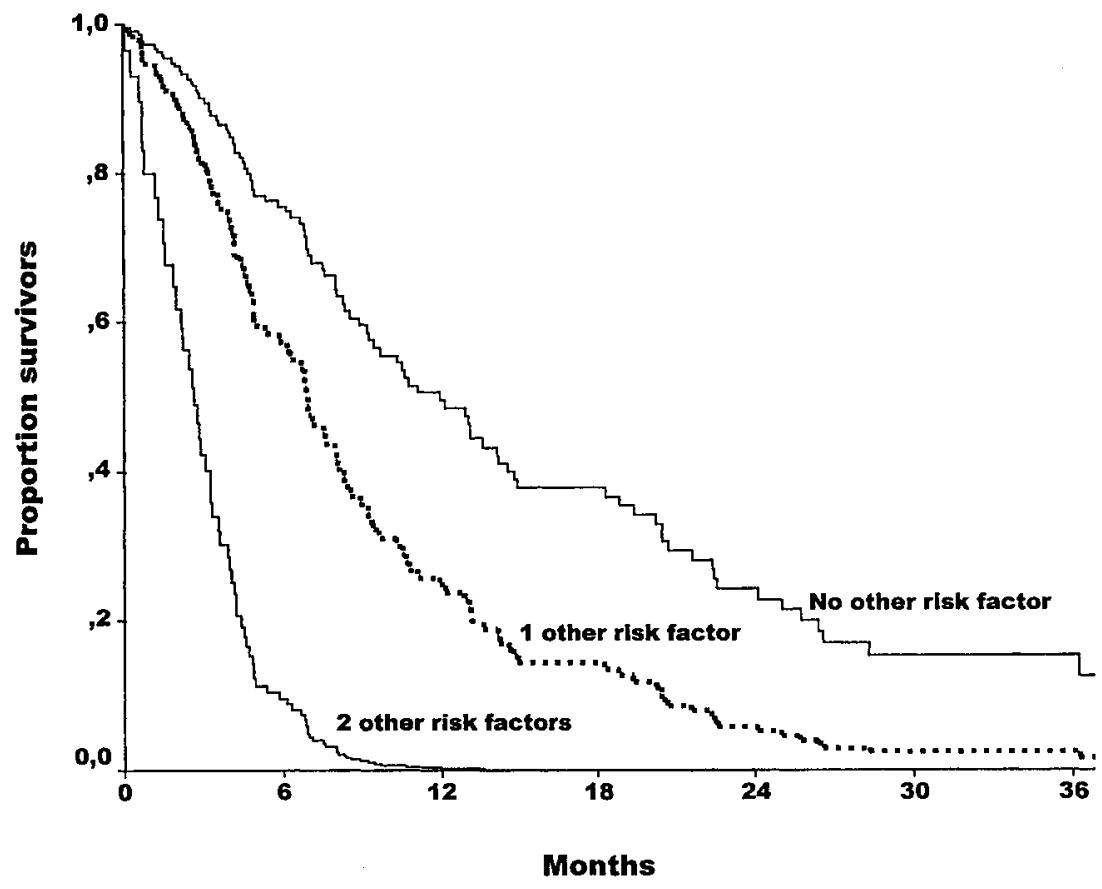

Independent risk factors are weight loss and global QoL.

Since a previous randomised study has shown a dose-response relationship for radiotherapy in NSCLC ${ }^{36}$, the choice of the radiotherapy regimen usually represents a compromise between an adequate dose and an acceptable length of treatment. In general, 
patients with favourable prognostic factors for survival will be selected for high dose radiotherapy in order to achieve long-term loco-regional tumour control. Low dose radiotherapy is considered more appropriate for patients with poor prognostic factors primarily in order to achieve palliation of respiratory symptoms and preserve or improve QoL. In view of the great individual variability in response to treatment, it is possible that some selected subgroups of patients benefit more from high dose radiotherapy than others. With the model in which only the non-QoL variables were entered, a group of patients could be identified with a median survival of 2.8 months and no survivors after 7.8 months, despite high dose radiotherapy. Although global QoL was the strongest prognostic indicator in the model in which QoL dimensions were entered as well, almost the same cohort of patients was identified as having poor outcome. Although in this study, the additional value of global QoL in identifying a group of patients with poor prognostic outcome remained limited; this QoL scale could be considered as an important stratification factor in clinical trials in which QoL is one of the endpoints.

In conclusion, global QoL is a strong prognostic factor for survival in patients with NSCLC who have pathological lymph nodes at presentation and who are treated with radical or curative radiotherapy. Additional studies are needed to confirm the prognostic significance of patient-rated QoL in this category of patients. 


\section{Q References}

1. Priestman TJ, Baum M. Evaluation of quality of life in patients receiving treatment of advanced breast cancer. Lancet 1976; 1: 899-901.

2. Spitzer WO, Dobson AJ, Hall J, et al. Measuring the quality of life of cancer patients. J Chron Dis 1981; 34: 585597.

3. Schipper $H$, Clinch J, McMurray $A$, et al. Measuring the quality of life of cancer patients. The Functional Living Index-Cancer: Development and validation. J Clin Oncol 1984; 2; 472-483.

4. Schag CA, Ganz PA and Heinrich RL. Cancer, Rehabilitation Evaluation System-short form (CARES-SF). A cancer specific rehabilitation and quality of life instrument. Cancer 1991; 68(6): 1406-13.

5. Aaronson NK, Ahmedzai S, Bergman B, et al. The European Organization for Research and Treatment of Cancer QLQ-C30: A quality of life instrument for use in international clinical trials in oncology. J Natl Cancer Inst 1993; 85: 365-376.

6. Te Velde A, Sprangers MA and Aaronson NK. Feasibility, psychometric performance, and stability across modes of administration of the CARES-SF. Ann Oncol 1996; 7(4): 381-90.

7. Coates A, Gebski V, Bishop JF, et al. Improving the quality of life during chemotherapy for advanced breast cancer. A comparison of intermittent and continuous treatment strategies. N Engl J Med 1987; 317: 1490-1495.

8. Kaasa S, Mastekaasa A and Naess S. Quality of life of lung cancer patients in a randomized controlled clinical trial evaluated by a psychosocial well-being questionnaire. Acta Oncol 1988; 27 : 335-342.

9. Simons JPFHA, Aaronson NK, Vansteenkiste JF, et al. Effects of Medroxyprogesterone Acetate on appetite, weight and quality of life in advanced stage non-hormone-sensitive cancer: A placebo-controlled multicenter study. $J$ Clin Oncol 1996; 14: 1077-1084.

10. Giaconne G, Splinter TAW, Debruyne C, et al. Randomized study of Paclitaxel-Cisplatin versus cisplatin-Teniposide in patients with advanced non-small cell lung cancer. J Clin Oncol 1998; 16: 2133-2141.

11. Cassileth BR, Lusk EJ, Miller DS, Brown LL and Miller C. Psychosocial correlates of survival in advanced malignant disease? N Engl J Med 1985; 312: 1551-5.

12. Kaasa $S$, Mastekaasa $A$ and Lund $E$. Prognostic factors for patients with inoperable non-small cell lung cancer, limited disease. The importance of patients' subjective experience of disease and psychosocial well being. Radiot Oncol 1989; 15: 235-242.

13. Ganz PA, Lee JJ and Siau J. Quality of life assessment: an independent prognostic variable for survival in lung cancer. Cancer $1991 ; 67 ; 3131-35$.

14. Coates $A$, Gebski V, Signorini $D$, et al. Prognostic value of quality of life scores during chemotherapy for advanced breast cancer. J Clin Oncol 1992; 10: 1833-1838.

15. Coates A, Thomson D, McLeod GRM, et al. Prognostic value of quality of life scores in a trial of chemotherapy with or without interferon in patients with metastatic malignant melanoma. Eur J Cancer 1993; 29A: 1731-34.

16. Earlam $S$, Glover $C$, Fordy $C$, et al. Relation between tumor size, quality of life, and survival in patients with colorectal liver metastases. J Clin Oncol 1996; 14: 171-175.

17. Coates $A$, Porzsolt $F$ and Osoba D. Quality of life in oncology practice: Prognostic value of EORTC QLQ-C30 scores in patients with advanced malignancy. Eur $\mathrm{J}$ Cancer 1997; 33 (7): 1025-1030.

18. Chang VT, Thaler HT, Polyak TA, et al. Quality of life and survival: The role of multidimensional symptom assessment. Cancer 1998; 83: 173-9.

19. Albain KS, Crowley JJ, LeBlanc $M$ and Livingstone RB. Survival determinants in extensive-stage non-small cell lung cancer: the Southwest Oncology Group Experience. J Clin Oncol 1991; 9: 1618-1626. 


\section{Chapter 4}

20. Lanzotti VJ, Thomas DR, Boyle LE, et al. Survival with inoperable lung cancer: an integration of prognostic variables based on simple clinical criteria. Cancer 1977; 39: 303-13.

21. Bonomi $P$, Gale $M$, Rowland $K$, et al. Pre-treatment prognostic factors in stage lli non-small cell lung cancer patients receiving combined modality treatment. Int J Radiat Oncol Biol Phys 1991; 20: 247-52.

22. Stanley KE. Prognostic factors for survival in patients with inoperable lung cancer. J Natl Cancer Inst 1980; 65: 2532.

23. Capewell S and Sudlow MF. Performance and prognosis in patients with lung cancer. Thorax 1990; 45: 951-956.

24. Sorensen JB, Badsberg JH and Olsen J. Prognostic factors in inoperable adenocarcinoma of the lung: a multivariate regression analysis of 259 patients. Cancer Res 1989; 49: 5748-54.

25. Jeremic $B$ and Shibamoto $Y$. Effect of interfraction interval in hyperfractionated radiotherapy with or without concurrent chemotherapy for stage III nonsmall cell lung cancer. Int J Radiat Oncol Biol Phys 1996; 34: 303-8.

26. Kupelian PA, Komaki $R$, Allen $P$, et al. Prognostic factors in the treatment of node-negative nonsmall cell lung carcinoma with radiotherapy alone. Int J Radiat Oncol Biol Phys 1996; 36: 607-13.

27. Nieder $C$, Nestle $U$, Ukena $D$, et al. Tumour markers as prognostic factors in non-small cell lung cancer. Strahlenther Onkol 1995; 171: 587-93.

28. PaterJL and Loeb M. Nonanatomic prognostic factors in carcinoma of the lung. Cancer 1982; 50: 326-331.

29. Ferrigno $D$ and Buccheri GF. A comprehensive evaluation of serum ferritin levels in lung cancer patients. Lung Cancer 1992; 8: 85-94.

30. In Hermanek P and Sobin LH (ed) In: TNM Atlas: Classification of malignant tumours; Springer-Verlag, 1992: 75-84.

31. Bjordal K, Kaasa S and Mastakaasa A: Quality of life in patients treated for head and neck cancer: A follow up study 7 to 11 years after radiotherapy. Int J Radiat Oncol Biol Phys 1994; 28: 847-856.

32. Niezgoda HE and Pater JL. A validation study of the domains of the core EORTC quality of life questionnaire. Qual Life Res 1993; 2: 319-325.

33. Cox DR and Oakes D: Analysis of survival data. London, United Kingdom, Chapman \& Hall, 1988.

34. Kaplan $E$ and Meier P: Non parametric estimation from incomplete observations. Am Stat Assoc J 1958; 53: $457-$ 481.

35. Peto $R$, Pike $M C$, Armitage $P$, et al. Design and analysis of randomized clinical trials requiring prolonged observation of each patient. II. Analysis and examples. Br J Cancer 1977; 35: 1-39.

36. Perez CA, Stanley K, Grundy $G$, et al. Impact of irradiation technique and tumour extend in tumour control and survival of patients with unresectable non-oat cell carcinoma of the lung. Cancer 1982; 50: 1091-1099. 
Chapter 5

\title{
Is the chest radiograph a reliable tool in the assessment of tumor response after radiotherapy in non-small cell lung carcinoma?
}

\author{
Hans Langendijk \\ Rob Lamers \\ Guul ten Velde \\ Dave Sanders \\ Jos de Jong \\ Fons Kessels \\ Miel Wouters
}

Published in:

International Journal of Radiation Oncology Biology and Physics 41: 1037-1045, 1998. 


\section{口 Abstract}

Background: The purpose of this study was to evaluate whether the chest radiograph is a reliable tool to assess response to radiotherapy.

Materials and methods: Pre- and post-treatment chest radiographs and computed tomographs (CT) of 63 patients with non-small cell lung cancer (NSCLC) treated by radiotherapy were reviewed by 4 observers with regard to suitability for tumor measurement, and response. Suitability for tumor measurement was expressed as the number of measurable diameters. In addition, the consequences to clinical outcome were studied by survival analysis.

Results: The CT turned out to be more suited for tumor measurement before as well as after radiotherapy, resulting in an increase of the number of measurable cases. The number of measurable cases with CT was $52(83 \%)$ as compared to $28(44 \%)$ with chest radiography. Especially in case of centrally localised tumors, the presence of an atelectasis or squamous cell carcinoma, CT contributed to a higher rate of measurable cases. The interobserver agreement with regard to response using chest radiograph was good (mean kappa $=0.74$ ). In 25 out of 28 cases ( $89 \%$ ) measurable with CT as well as with chest radiograph response was equally classified. When CT was used, the median survival of the responders was 14.2 months as compared to 6.8 months of the non-responders. When chest radiograph was used the median survival of these groups was 12.0 and 6.6 months respectively, which was not significantly different when response was assessed by $\mathrm{CT}$.

Conclusion: We conclude that CT is more suited for tumor measurement because more measurable lesions can be found and more evaluable lesions on chest radiograph become measurable on CT. The chest radiograph does have a valuable role to play in those lesions that are measurable because of the good interobserver agreement with regard to the response classification, the high overall agreement between $\mathrm{CT}$ and chest radiograph in case of measurable cases and the lack of important differences with regard to the survival. 


\section{Introduction}

In inoperable non-small cell lung carcinoma (NSCLC) irradiation has been widely used to achieve definitive tumor control and/or palliation of symptoms. In many studies concerning inoperable NSCLC tumor response is an important endpoint. Usually, tumor response is defined as a reduction of the product of 2 perpendicular diameters with $50 \%$ or more and measured by computed tomography of the chest (CT) ${ }^{1-7}$. In the Netherlands, a CT after radiotherapy is not a routine procedure in patients who do not take part in a prospective trial. Therefore, the assessment of tumor response in retrospective analyses is usually made by chest radiograph. The present study was conducted to investigate whether chest radiograph is a reliable tool in the assessment of tumor response to radiotherapy and deals with three issues related to response assessment. Firstly, the suitability of the chest radiograph and the CT for tumor measurement and for response assessment was analyzed. Secondly, the interobserver variability of the chest radiograph regarding tumor response assessment was investigated and thirdly the validity towards response assessment of the chest radiograph was studied, assuming the CT to be gold standard in the assessment of tumor response. Finally, the consequences for the clinical outcome were studied by survival analysis.

\section{- Patients and methods}

\section{Patients selection}

From June 1994 to December 199563 patients with inoperable NSCLC were consecutively included. Diagnosis was confirmed by histology and/or cytology in all patients. Patients were referred to the RTIL to be treated by locoregional irradiation. In all patients chest radiographs and $\mathrm{CT}$-examinations before and after radiotherapy were available. There were 54 males ( $86 \%$ ) and 9 females (14\%). Patients age ranged from 37 to 83 years (median: 67 years). Twenty-eight cases (44\%) were classified as T1-T2 and $35(56 \%)$ as T3-T4 ${ }^{8}$. Sixteen out of the 28 patients with a T1 or T2 tumor were inoperable because of positive lymph nodes found with mediastinoscopy $(n=13)$ or because of enlarged lymph nodes asessed with CT which were regarded as pathological $(n=3)$. There were 12 patients with T1 or T2 tumors, classified as N0, who were considered inoperable because of medical 
reasons, such as impaired lung function or cardiac disease. In this series, 16 patients had T3 tumors. Thirteen patients had centrally localized tumors, classified as T3 because of extension into the main bronchus less than $2 \mathrm{~cm}$ from the main carina and/or complete atelectasis of one lung. Three patients were classified as T3 because of extension into the chest wall. Of the 19 patients with T4 tumors, 18 subjects had a centrally localized tumor with extension into mediastinal structures. One patient was classified as T4 because of extension into the vertebral corpus. Forty-one cases were classified as $\mathrm{N}+$ based on enlarged lymph nodes detected with CT-examination or based on positive mediastinoscopy. In 22 patients a mediastinoscopy was performed. In 4 of these patients, no enlarged lymph nodes were found on CT. In the other 18 cases, there were enlarged lymph nodes on CT as well. Patients with distant metastases before radiotherapy were excluded. The histologic type included squamous cell carcinoma in 42 cases $(67 \%)$, adenocarcinoma in 8 cases $(13 \%)$ and large cell carcinoma in 13 cases $(21 \%)$. All patients were treated with radiotherapy alone and no chemotherapy had been administered previously. At our department, the standard policy in inoperable NSCLC is radiotherapy.

\section{Radiotherapy}

External beam radiotherapy was administered in all patients. Fifty-five patients (87\%) were treated with high dose radiotherapy (group 1) and 8 patients (13\%) were treated with low dose radiotherapy (group 2). Inoperable subjects with a WHO performance of 0 to 2 , without supraclavicular lymph node metastases or distant metastases were treated with high dose radiotherapy. The remaining patients were treated with low dose radiotherapy. Patients with a WHO performance of 4 were not treated. In group 1, the primary tumor, the mediastinal lymph nodes and the ispilateral hilus were irradiated up to a total dose of 45 Gray (Gy) in daily fractions of 2.25 Gy (4 times a week). A booster was given to the primary tumor and enlarged mediastinal lymph nodes up to a cumulative dose of $60 \mathrm{~Gy}$ using a fraction dose of $2.50 \mathrm{~Gy}$ (4 times a week). In group 2 the primary tumor, mediastinal lymph nodes and ipsilateral hilus were irradiated up to a total dose of $30 \mathrm{~Gy}$ in daily fractions of 3.0 Gy (4 times a week).

In 16 patients (25\%) endobronchial brachytherapy was administered as well. Two fractions of $7.5 \mathrm{~Gy}$ calculated at a $1 \mathrm{~cm}$ distance from the central axis were given to the endobronchial tumor at day 1 and 8 of the treatment course. Patients treated with brachytherapy had 
endobronchial and obstructive tumors in the proximal airways and took part in a randomized trial in which the additional value of brachytherapy to external beam irradiation was investigated.

\section{Chest radiograph and $\mathrm{CT}$ imaging}

The chest radiograph and the $\mathrm{CT}$ made during the diagnostic work up were used as baseline investigations. All study subjects had undergone postero-anterior and lateral chest radiography. Follow up chest radiographs and CT-examinations were performed between 2 and 4 weeks after radiotherapy. All patients in the present study also took part in a prospective quality of life study investigating changes in respiratory symptoms en quality of life following radiotherapy. One of the aims of this quality of life study was to investigate whether changes in quality of life and respiratory symptoms correlated with an objective response as observed on chest radiograph and CT. For this purpose, quality of life was assessed before as well as 2 to 4 weeks after radiotherapy and therefore it was decided to perform a chest radiograph and CT in the same period.

Scans were photographed with window settings appropriate for evaluation of the mediastinum (400/50) and lung parenchyma (1500/-550). The slice thickness was 1 centimeter and contrast enhancement was used in almost all cases.

\section{Interpretation of $C R$ and $C T$}

The 126 chest radiographs and $126 \mathrm{CT}$ examinations were interpreted in separate sessions. In the first session the chest radiographs before and after radiotherapy of each individual patient were reviewed by the 4 observers independently. The baseline and follow up chest radiographs were reviewed simultaneously. In the second session the same procedure was followed with the $\mathrm{CT}$. The only clinical information to the observers was that all patients were treated with radiotherapy in between.

Tumor diameters of the primary tumors of each examination were measured and scored in 3 directions if possible (transversal, craniocaudal, anterio-posterior). The diameters of enlarged lymph nodes were not taken into account. For each examination suitability for tumor measurement was scored using a 4-point scale ( $3=$ three diameters measurable, $2=$ two diameters measurable, $1=$ one diameter measurable, $0=$ no diameter measurable). 
Figure 1: Example of a case with measurable disease on CT scan of the chest. CT scan before radiotherapy through the aortic arch shows a mass in the right upper lobe measurable in at least 2 directions. There was a decrease volume in the right lung and a lobulated mass in the right upper lobe abutting the mediastinum. Note that there is an ipsilateral enlarged paratracheal lymph node.

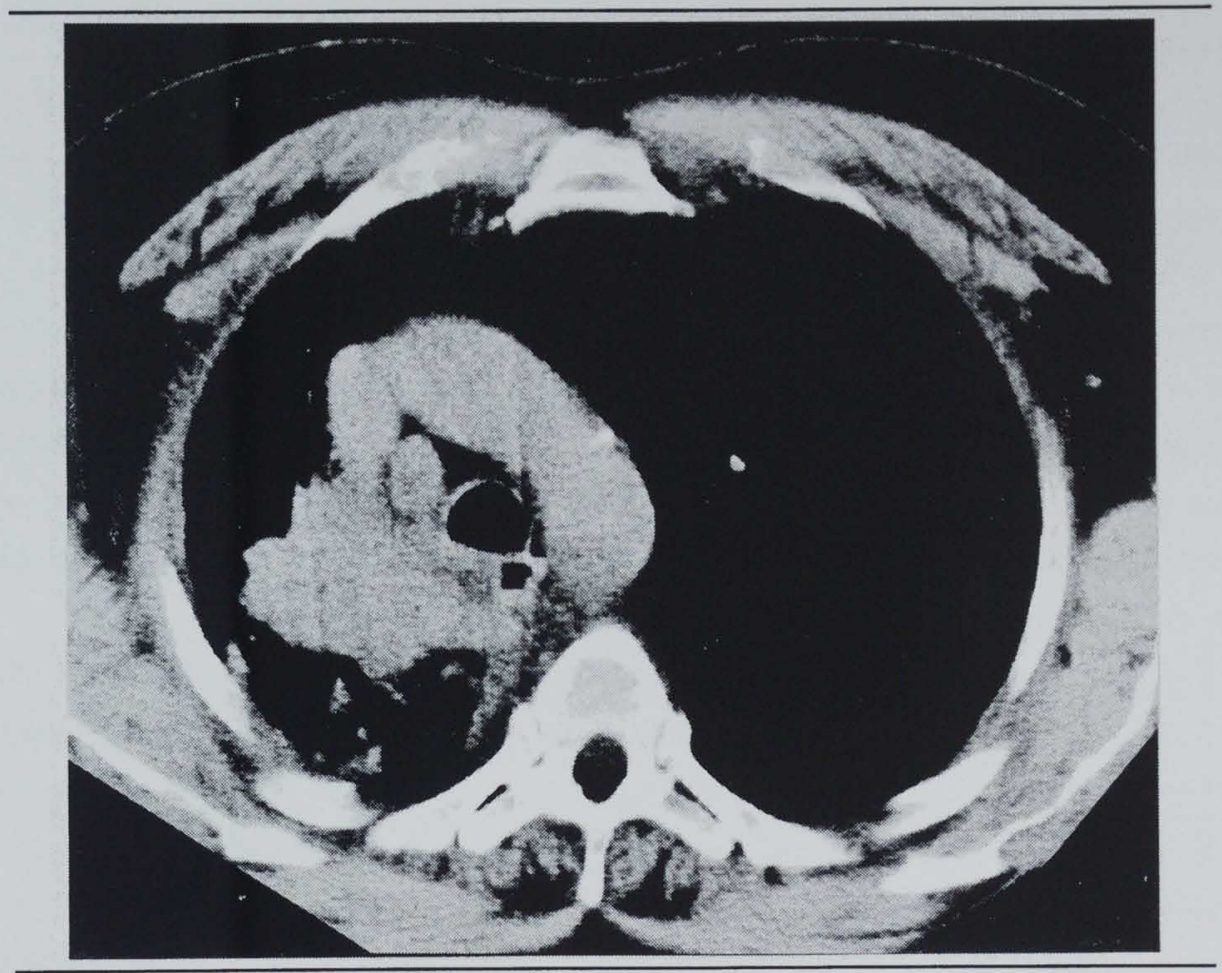

\section{Response assessment}

To assess response the product of the 2 largest diameters measured was calculated before and after radiotherapy. The percentage of reduction was assessed for each pair of chest radiographs and CT examinations and for each observer using a computer program and was calculated after the interpretation procedure for all observers was completed. A complete response was defined as a complete disappearance of the primary tumor. A partial response was defined as a reduction of $50 \%$ or more of the product of the 2 largest diameters. A reduction of less than $50 \%$ or progression less than $25 \%$ was classified as stable disease. An increase of more than $25 \%$ was classified as progressive disease. 
Figure 2: CT-scan of the chest through the aortic arch of the same patient as shown in figure 1 allows measurement in at least 2 directions of the tumour after radiotherapy as well. This patient had measurable disease and was classified as responder.

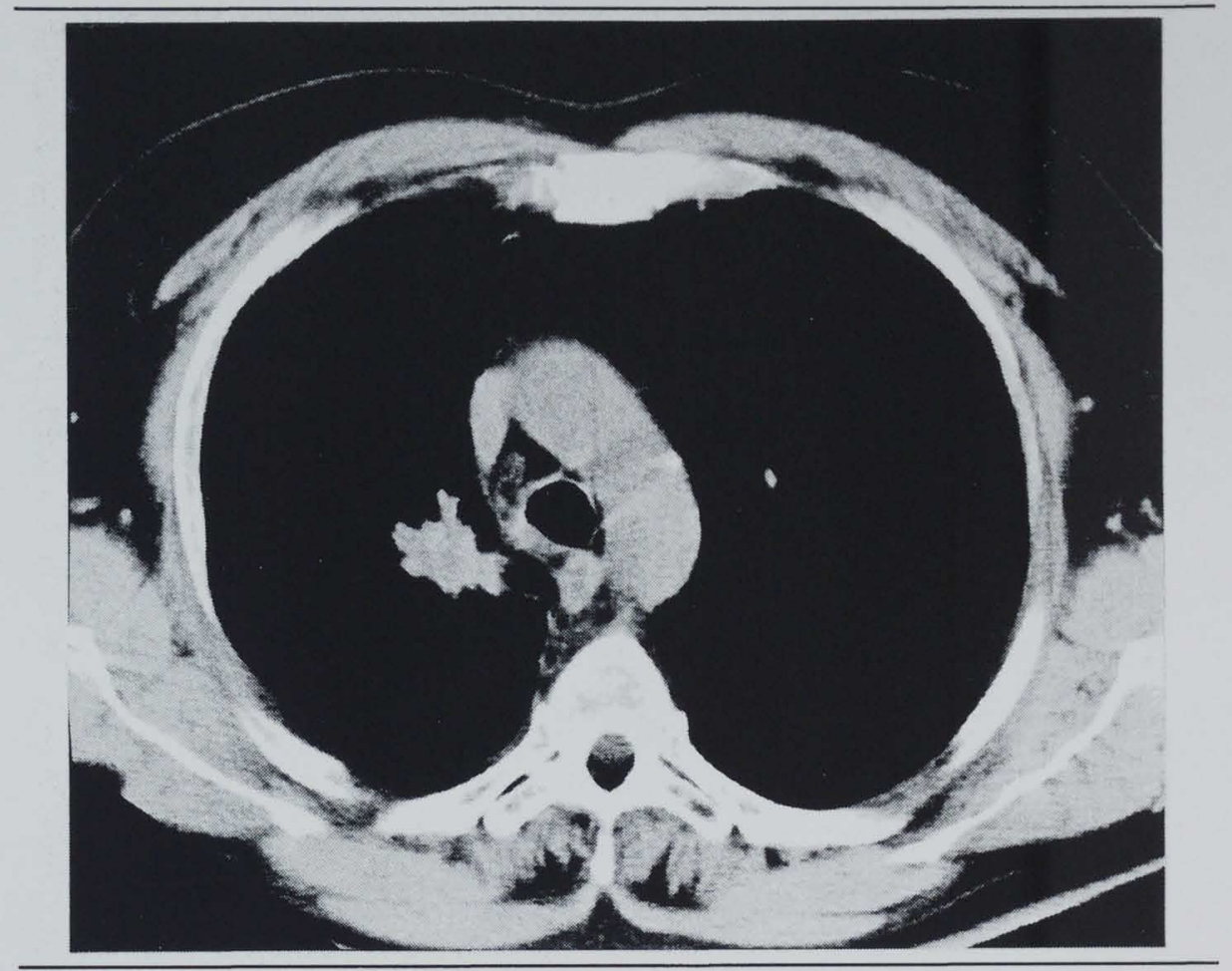

Response assessment was classified as measurable when it was possible to measure at least 2 diameters before and after radiotherapy (Figure 1 and 2). When it was not possible to measure at least two diameters but an obvious improvement, complete disappearance or clear progression of the abnormalities was noted by the observer, response assessment was defined as evaluable. Examples of obvious improvement are the complete disappearance of an atelectasis or a clear reduction of a centrally localised and thus partly visible tumor which could not be measured in 2 directions (Figure 3 and 4). After the first analysis a session was organised for those examinations, in which major disagreement regarding response classification had been noted between the 4 observers, in order to make an overall response classification for all four observers together. 
Figure 3: Posteroanterior chest radiograph before radiotherapy of the same patient as shown in figures 1 and 2 shows massive air-space consolidation throughout the right lung. Tumour measurement was not possible.

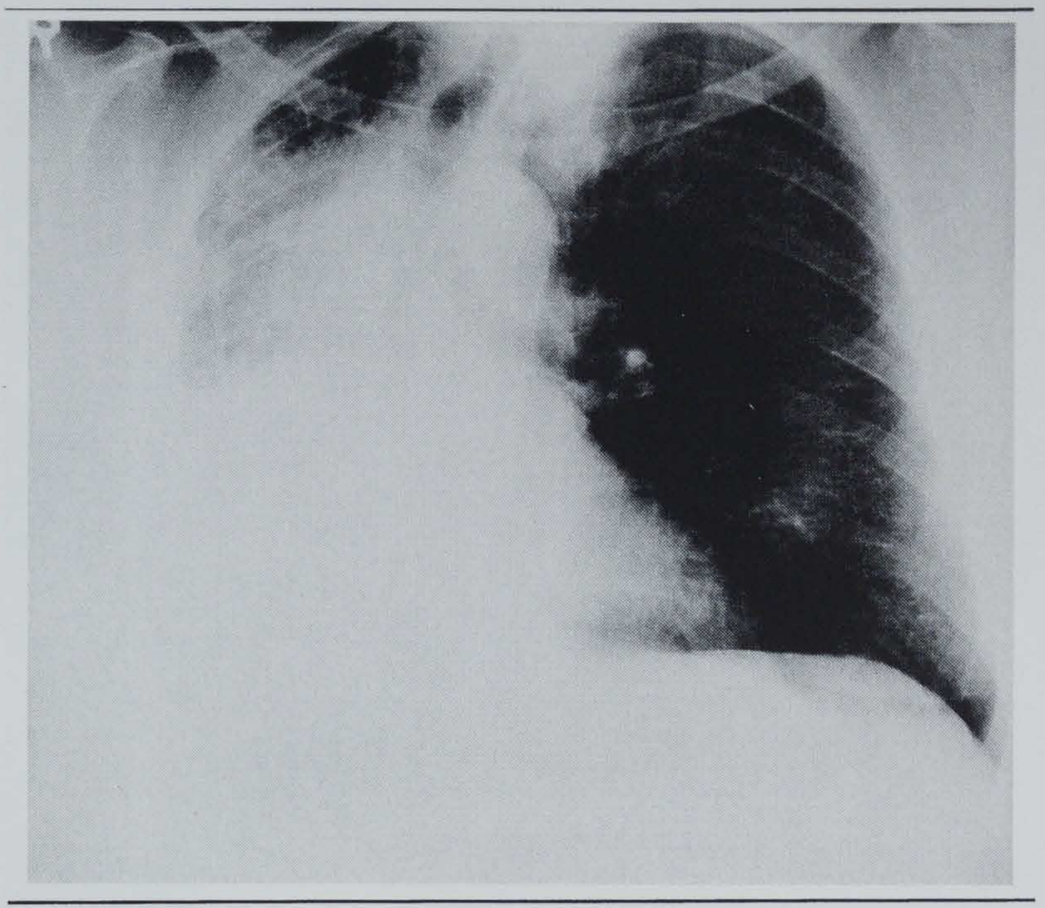

\section{Statistics}

The interobserver agreement was calculated and expressed in unweighted kappa-values. The unweighted kappa is a measurement of agreement in excess of what is expected by chance and is calculated according to the formula $k=\left(P_{0}-P_{e}\right) /\left(1-P_{e}\right)$, in which $P_{0}$ represents the observed and $\mathrm{P}_{\mathrm{e}}$ the expected proportion of agreement. Percent agreement and kappavalues were calculated for each couple of observers ${ }^{9,10}$. To analyze the degree of suitability the mean suitability score (MSS) for each examination was computed by calculating the mean of the suitability scores of the four individual observers, resulting in a linear scale from 0 to 3 in which lower scores represent worse suitability. A stepwise multivariate analysis was performed to identify independent predictive factors towards the degree of suitability.

To investigate the validity of the chest radiograph towards response assessment the CT was regarded as gold standard. Percent agreement, negative predictive values and positive 
Figure 4: Posteroanterior chest radiograph of the same patient after radiotherapy. The chest radiograph shows a clearance of the massive atelectasis as was observed before radiotherapy. There is an irragular mass in the upper border of the right hilum. This case was considered evaluable with chest radiograph and classified as responder.

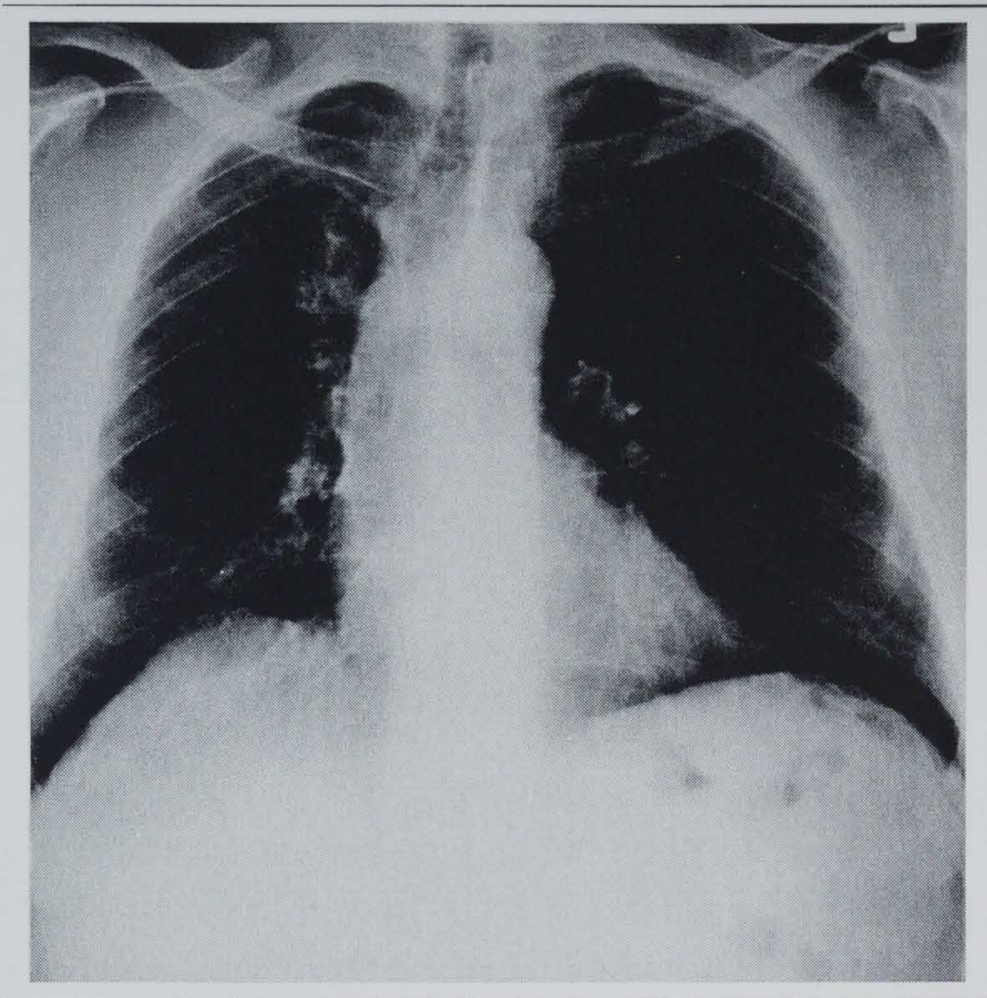

predictive values of the chest radiograph were calculated for the overall response classification of the chest radiograph and CT after the panel discussion.

The Kaplan-Meier method was used to calculate the overall survival and the log rank test was used to test differences between curves. 


\section{Results}

\section{Suitability for tumor measurement}

The MSS before radiotherapy was significantly better as compared to after radiotherapy in both chest radiograph and CT. The MSS of the CT was significantly better than the chest radiograph before as well as after radiotherapy (Table 1).

Table 1: Mean suitability score before and after radiotherapy

\begin{tabular}{lcc}
\hline Investigation & Mean suitability score \\
\cline { 2 - 3 } & Before radiotherapy & After radiotherapy \\
& $(95 \%-\mathrm{Cl})$ & $(95 \%-\mathrm{Cl})$ \\
\hline Chest radiopgraph & $1.5(1.2-1.8)$ & $1.0(0.7-1.3)$ \\
CT-scan of the chest & $1.7(1.5-2.0)$ & $2.2(1.9-2.5)$ \\
\hline All differences were statistically significant $(p<0.01)$ &
\end{tabular}

The correlation of a number of variables with the degree of suitability was investigated. These variables were histology (squamous cell carcinoma versus non-squamous cell carcinoma), the presence of an atelectasis (present versus not present), localization (peripheral versus central), T-stage (T1-T2 versus T3-T4), sex (male versus female), age ( $0-70$ years versus $>70$ years), site (left versus right), external beam radiotherapy ( $30 \mathrm{~Gy}$ versus $60 \mathrm{~Gy}$ ) and endobronchial brachytherapy (yes versus no). In the multivariate analysis with regard to the degree of suitability after radiotherapy the presence of changes due to radiotherapy (yes or no) were taken into account as well.

After performing a multivariate regression analysis regarding the degree of suitability of the chest radiograph, central localization of the tumor, squamous cell carcinoma and the presence of atelectasis turned out to be independent factors predicting worse suitability for tumor measurement at chest radiograph before radiotherapy. Central localisation and atelectasis contributed to a worse suitability after radiotherapy as well (Table 2). 
Table 2: Suitability for tumour measurement of the chest radiograph: results of the multivariate analysis.

\begin{tabular}{|c|c|c|c|c|c|c|c|c|c|}
\hline \multirow[t]{3}{*}{ Variables } & \multirow{3}{*}{$\begin{array}{c}\text { Number of } \\
\text { patients }\end{array}$} & \multicolumn{4}{|c|}{ Chest radiograph before radiotherapy } & \multicolumn{4}{|c|}{ Chest radiograph after radiotherapy } \\
\hline & & \multirow{2}{*}{$\begin{array}{c}\text { Mean } \\
\text { suitability } \\
\text { score }\end{array}$} & \multicolumn{3}{|c|}{ Multivariate analysis } & \multirow{2}{*}{$\begin{array}{l}\text { Mean } \\
\text { suitability } \\
\text { score }\end{array}$} & \multicolumn{3}{|c|}{ Multivariate analysis } \\
\hline & & & Coefficient & SE & p-value & & Coefficient & SE & p-value \\
\hline \multicolumn{10}{|l|}{ Localisation } \\
\hline Central & 49 & 1.2 & 0.97 & 0.27 & $p<0.001$ & 0.8 & 0.89 & 0.26 & $p=0.001$ \\
\hline Peripheral & 14 & 2.5 & & & & 1.8 & & & \\
\hline \multicolumn{10}{|l|}{ Histology } \\
\hline $\operatorname{SCC} * *$ & 42 & 1.2 & 0.67 & 0.21 & $p=0.006$ & 0.8 & \multicolumn{3}{|c|}{ not significant } \\
\hline Non-SCC & 21 & 2.1 & & & & 1.4 & & & \\
\hline \multicolumn{10}{|l|}{ Atelectasis } \\
\hline None & $30(26)^{\star}$ & 2.3 & 0.25 & 0.10 & $p=0.017$ & 1.6 & 0.32 & 0.10 & $p=0.002$ \\
\hline Lobar & $33(30)^{\star}$ & 1.0 & & & & 1.0 & & & \\
\hline Entire lung & $4(3)^{*}$ & 0 & & & & 0 & & & \\
\hline
\end{tabular}

* The number in brackets refers to the number of patients after radiotherapy

** SCC = squamous cell carcinoma

Table 3: Suitability for tumour measurement of the CT of the chest: results of the multivariate analysis.

\begin{tabular}{|c|c|c|c|c|c|c|c|c|c|}
\hline \multirow[t]{3}{*}{ Variables } & \multirow{3}{*}{$\begin{array}{l}\text { Number of } \\
\text { patients }\end{array}$} & \multicolumn{4}{|c|}{ Chest radiograph before radiotherapy } & \multicolumn{4}{|c|}{ Chest radiograph after radiotherapy } \\
\hline & & \multirow{2}{*}{$\begin{array}{c}\text { Mean } \\
\text { suitability } \\
\text { score }\end{array}$} & \multicolumn{3}{|c|}{ Multivariate analysis } & \multirow{2}{*}{$\begin{array}{c}\text { Mean } \\
\text { suitability } \\
\text { score }\end{array}$} & \multicolumn{3}{|c|}{ Multivariate analysis } \\
\hline & & & Coefficient & SE & p-value & & Coefficient & SE & p-value \\
\hline \multicolumn{10}{|l|}{ Atelectasis } \\
\hline None & $26(30)^{\star}$ & 3.0 & 0.62 & 0.07 & $p<0.001$ & 2.7 & 1.04 & 0.26 & $p<0.001$ \\
\hline Lobar & $33(30)^{\star}$ & 2.4 & & & & 2.0 & & & \\
\hline Entire lung & $4(3)^{*}$ & 0.6 & & & & 0.6 & & & \\
\hline \multicolumn{10}{|l|}{ Histology } \\
\hline $\mathrm{SCC}^{* *}$ & 42 & 2.3 & 0.42 & 0.16 & $p=0.01$ & 2.1 & \multicolumn{3}{|c|}{ not significant } \\
\hline Non-SCC & 21 & 2.9 & & & & 2.3 & & & \\
\hline
\end{tabular}

"The number in brackets refers to the number of patients after radiotherapy

* SCC = squamous cell carcinoma

In the multivariate analysis concerning the degree of suitability of the $\mathrm{CT}$, the presence of atelectasis was related with a worse degree of suitability before and after radiotherapy. Before radiotherapy the degree of suitability depended on histology as well (Table 3 ). 


\section{Response assessment}

After the panel discussion the chest radiograph was classified as measurable in 28 cases $(44 \%)$, evaluable in 13 cases $(21 \%)$ and not evaluable in 22 cases (35\%), which was significantly worse as compared to the CT in which 52 cases (83\%) were classified as measurable, 5 cases as evaluable $(8 \%)$ and 6 cases $(10 \%)$ as not evaluable $(P<0.001)$.

Of the 22 subjects not evaluable with the chest radiograph, 14 cases were classified as measurable with CT (64\%), 2 cases as evaluable with CT (9\%) and 6 cases $(27 \%)$ remained not evaluable. These 6 cases were not evaluable because of severe atelectasis, which made it impossible to distinguish between tumor and collapsed lung even with CT.

In case of the presence of factors contributing to a significantly worse degree of suitability for tumor measurement the use of CT resulted in a decrease of the number of nonevaluable cases (Figure 5).

Interobserver agreement with regard to response classification of the chest radiograph When only measurable cases were taken into account the unweigthed kappa values of each combination of observers ranged from 0.51 to 1.00 (mean: 0.74 ). When the measurable as well as the evaluable cases were taken into account the unweighted kappa values ranged from 0.61 to 0.83 (mean: 0.69 ).

\section{Response classification}

With CT 3 lesions out of $57(5 \%)$ were classified as complete response, 36 lesions (63\%) as partial response, 14 lesions (25\%) as stable disease and 4 lesions (7\%) as progressive disease. With chest radiography 1 lesion out of $41(2 \%)$ was classified as complete response, 22 lesions (54\%) as partial response, 12 lesions (29\%) as stable disease and 6 lesions (15\%) as progressive disease. These percentages did not differ significantly.

\section{Validity}

In table 4 only the 28 patients who were measurable with CT as well as with chest radiography are mentioned. In 25 out of 28 cases ( $87 \%)$ the response assessed with chest radiograph and CT did not differ. Ten cases, classified as evaluable on chest radiograph, became measurable on CT. In 8 cases this classification was equal, however, 2 cases were 
Figure 5: Suitability for response assessment in factors significantly related to suitability

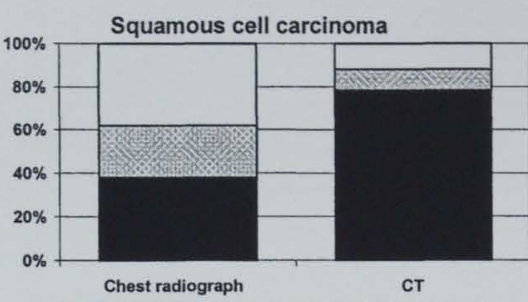

Measurable $\square$ Evaluable $\square$ Not evaluable
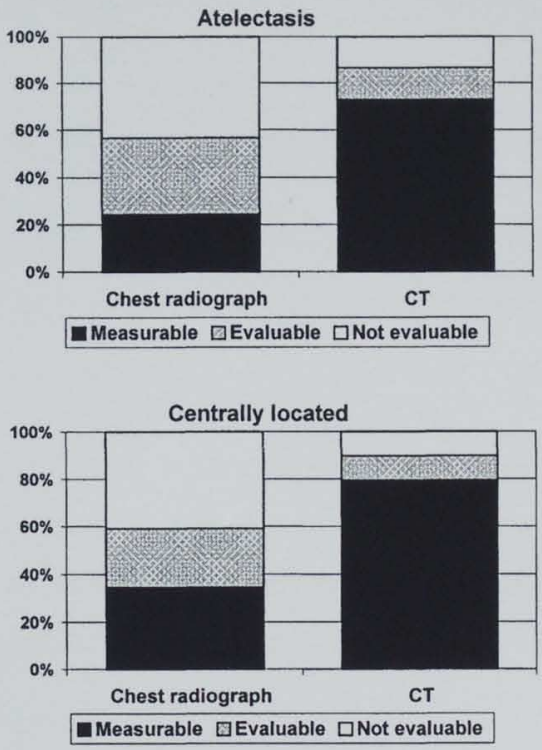
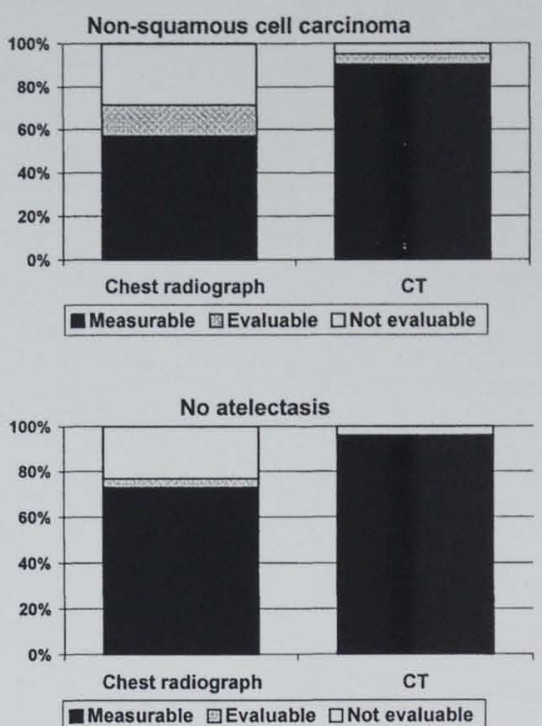

Measurable $\square$ Evaluable $\square$ Not evaluable

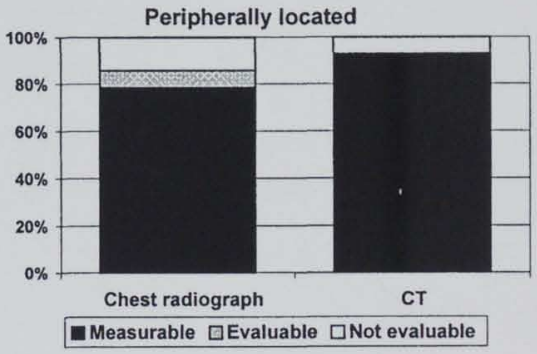

classified as progressive disease on chest radiograph, while they were classified as partial response on $\mathrm{CT}$.

To calculate the positive and negative predictive value the CT was considered to be gold standard and the patients were divided into two groups. The cases showing a complete or partial response were classified as responders and the patients showing stable disease or progressive disease as non-responders. Taking into account only measurable cases the overall agreement between chest radiograph and CT was $93 \%$. When the CT was considered as gold standard the positive predictive value (PPV) of the chest radiograph was $94 \%$ and the negative predictive value (NPV) was $92 \%$. When the measurable as well as the 
Table 4: Response classification of measurable cases with $\mathrm{CT}$ as well as with chest radiography

\begin{tabular}{lccccc}
\hline Chest radiograph & \multicolumn{5}{c}{ CT-scan of the chest } \\
\cline { 2 - 5 } & $\begin{array}{l}\text { Complete } \\
\text { response }\end{array}$ & $\begin{array}{c}\text { Partial } \\
\text { response }\end{array}$ & $\begin{array}{c}\text { Stable } \\
\text { disease }\end{array}$ & $\begin{array}{c}\text { Progressive } \\
\text { disease }\end{array}$ & Total number \\
\hline Complete response & 0 & 1 & 0 & 0 & $1(4 \%)$ \\
Partial response & 0 & 14 & 1 & 0 & $15(54 \%)$ \\
Stable disease & 0 & 1 & 8 & 0 & $9(32 \%)$ \\
Progressive disease & 0 & 0 & 0 & 3 & $3(11 \%)$ \\
\hline Total number & 0 & $16(57 \%)$ & $9(32 \%)$ & $3(11 \%)$ & 28 \\
\hline
\end{tabular}

evaluable cases were taken into account the PPV was $96 \%$ and the NPV was $78 \%$ with an overall agreement of $93 \%$.

\section{Survival}

The median survival of the group of patients classified as responders with the chest radiograph was 14.2 months and 12.0 months when measured with CT (Figure 6). The median survival of the patients clasified as non-responder with chest radiograph was 6.8 months and 6.6 months when measured with CT (Figure 7). These differences were not statistically different.

\section{Discussion}

This report discusses the results of a study, conducted in order to find out whether the chest radiograph is a reliable tool to assess response to radiotherapy, for instance in retrospective analyses.

The first step in the process of response assessment is the measurement of the tumor before and after radiotherapy. The CT turned out to be more suited for tumor measurement than the chest radiograph before as well as after radiotherapy, resulting in a lower proportion of patients not evaluable for response when CT was used. Lyn and coworkers reported on a comparison of chest radiographs and CT scans in the assessment of tumor 
Figure 6: Overall survival in the responders according to examination.

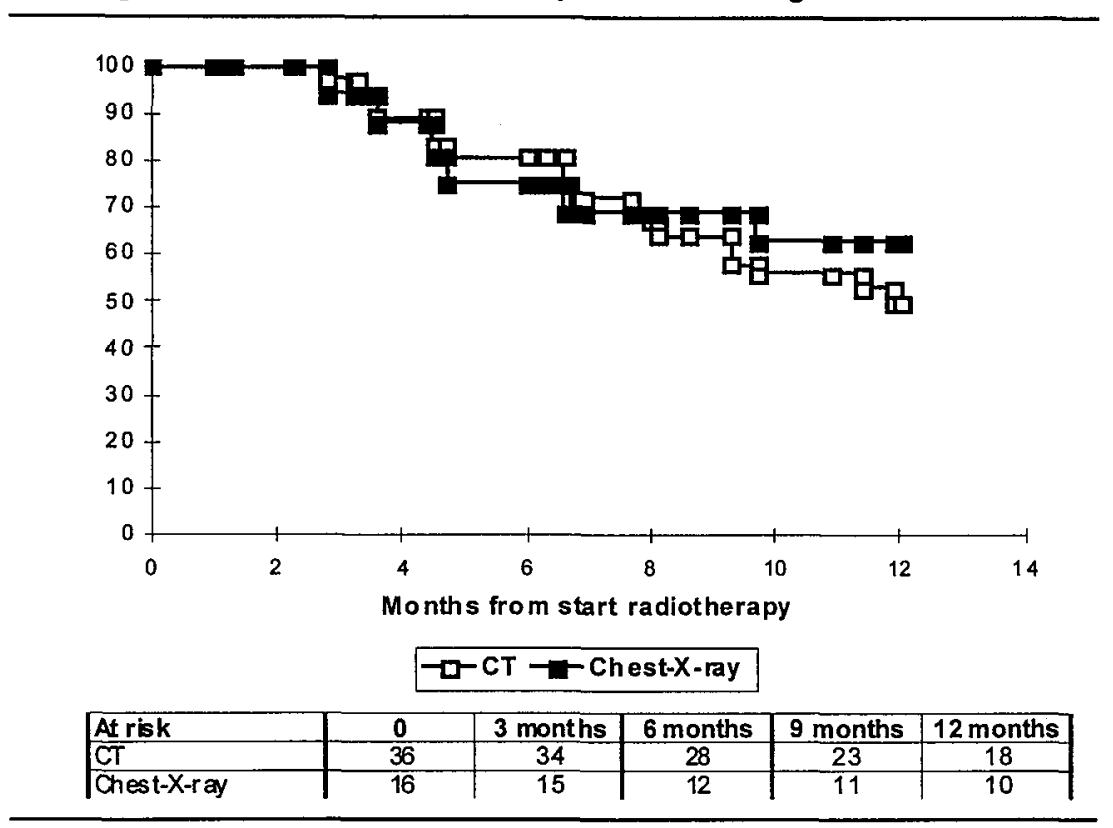

response in patients with lung cancer treated with CHART ${ }^{11}$. They showed that 14 out of 58 chest radiographs $(24 \%)$ turned out to be unsatisfactory for the assessment of response as compared to 2 out of 58 CT scans (3\%). Dajczman and coworkers ${ }^{12}$ compared response evaluation in small cell lung cancer using $\mathrm{CT}$ and chest radiograph. The number of cases in which neoplastic disease was measurable in two cross-sectional diameters on pretreatment films was $79 \%$ on chest radiograph and $97 \%$ on CT. In the present study $44 \%$ of the chest radiographs were classified as measurable in two cross-sectional diameters, which is considerably worse as compared to these two other studies. A possible explanation for this difference is that a high proportion of the patients in the present study had centrally localized tumors $(78 \%)$ with $(41 \%)$ or without atelectasis $(59 \%)$, both factors contributing to a worse degree of suitability.

The suitability for tumor measurement was significantly worse after radiotherapy as compared to before radiotherapy, This phenomenon was observed in the chest radiograph as well as in the CT of the chest. In the present study, the findings of just one post-treatment investigation, made 2 to 4 weeks after completion of radiotherapy were used to assess 
Figure 7: Overall survival in the non-responders according to examination.

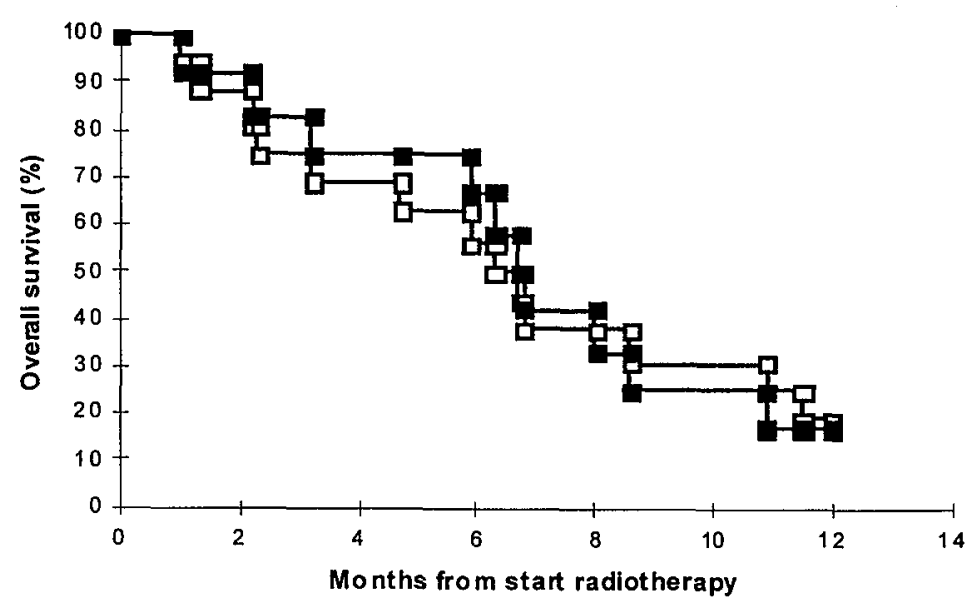

\begin{tabular}{|c|c|c|c|c|c|}
\hline & & \multicolumn{2}{|c|}{$\rightarrow-C T \rightarrow-C h e s t-X-$ ray } & & \\
\hline At risk & 0 & 3 months & 6 months & 9 months & 12 months \\
\hline $\mathrm{CT}$ & 16 & 12 & 9 & 5 & 3 \\
\hline ChestX-ray & 12 & 10 & 8 & 3 & 2 \\
\hline
\end{tabular}

response. Possibly, the assessment of tumor response become more difficult when the interval between the end of radiotherapy and the diagnostic investigations increases due to radiation-induced changes. Furthermore, in some slowly responding tumors, the reduction of the tumor will still go on beyond the selected interval, pointing out that the results of the present study only account for the selected interval. On the other hand, when serial investigations after radiotherapy are used to assess response, response assessment may become less difficult as it allows comparison of changes through time.

In the multivariate analysis, the presence of atelectasis and central localisation of the tumor contributed independently to a worse degree of suitability before radiotherapy when the chest radiograph was used. In these situations CT contributed to an important increase of measurable cases. The majority of cases classified as evaluable or not evaluable with chest radiograph became measurable with $\mathrm{CT}$. This phenomenon was particularly observed in case of centrally localized tumors and in the presence of an atelectasis. In centrally localized lesions the projection of normal structures in the mediastinum or atelectasis often precludes proper measurement of the tumor. With $\mathrm{CT}$, the cross-sectional orientation and 
the increased contrast between normal structures and tumor leads to better suitability, although 6 patients with severe atelectasis remained unevaluable with CT. The question rises whether other radiologic imaging techniques could be used to increase the number of measurable cases, e.g. by differentiating tumor from postobstructive lung collapse. Onitsuka et al. showed that rapid sequence CT with bolus injection of nonionic contrast medium was able to discriminate collapsed lung from tumor because of differences in enhancement between collapsed lung and tumor and secondly because of differences in internal structure observed with this technique ${ }^{13}$. An other solution could be magnetic resonance imaging (MRI) of the chest. Clinical MRI studies of lung cancer showed that obstructive atelectasis was found to have high signal intensity on T2-weighted images ${ }^{14-16}$. In $44 \%-75 \%$ of the cases reported, the atelectasis displayed higher signal intensity than the tumor, allowing a better differentiation between these two entities. Thus, heavily T2-weighted MR sequences may be helpfull in case contrast-enhanced CT is not suited for tumor measurement. Studies concerning the efficacy of MRI and CT in the staging of lung cancer determined that $C T$ and $M R I$ are approximately equal in the staging of N2 disease and that MRI is probably better in the assessment of superior sulcus tumors, tumors involving the aorta-pulmonary window, hilar nodes and in assessing chest wall or diaphragmatic invasion ${ }^{14}$. However, it remains unclear whether MRI contributes to a better degree of suitability for tumor measurement after conventional radiotherapy.

In view of the higher degree of suitability of the CT of the chest it was not surprising that the number of measurable cases used was significantly higher when CT was as compared to the number of measurable cases when chest radiograph was used. The group of cases who were classified as evaluable is a rather difficult group. Most of these patients had centrally localised tumors, which made it impossible to measure the tumor in at least 2 directions, but which showed a clear reduction on the chest radiograph. In a small number of patients classified as evaluable a reaeration of an atelectasis was noted. It has to be taken in to account that clearing of an atelectasis does not necessarily mean that the tumor is sufficiently smaller to be considered a responder. A mucous plug, blood, extraluminal fibrosis and other causes of bronchial obstruction may be the source for the abnormal findings. The same holds true for opacities due to pneumonia, pulmonary oedema and lymphangetic spread. In contrast, persistent atelectasis does not necessarily mean that there is no tumor response. In the present study, this problem was illustrated by the 2 
evaluable cases, classified as progressive disease on chest radiograph, while they were classified as partial responder on CT. In view of the differences with regard to the degree of suitablity it is preferable to use $\mathrm{CT}$ in response assessment resulting in a higher proportion of measurable lesions. However, in case of measurable disease on the chest radiograph, chest radiograph is a reliable method to assess response after radiotherapy.

The strength of agreement with regard to response assessment of the chest radiograph was classified as good in case only measurable cases were taken into account as well as in case measurable and evaluable cases were taken into account. The overall agreement between CT and chest radiograph was high in the measurable cases as well as in the evaluable cases, resulting in high negative and positive predicting values of the chest radiograph.

Lyn and coworkers reported on the value of chest radiography and computed tomography to assess response of lung cancer treated with radiotherapy. Of the 58 patients included in this study 44 patients were evaluable for response with chest radiography as well as with CT. The overall agreement between chest radiograph and CT scan was $86 \%$ (38/44), almost equal to our study ${ }^{11}$.

In a number of studies, response to irradiation was correlated with better survival ${ }^{17-20}$. In the present study the overall survival was better in the responders as compared to the nonresponders and the results with regard to the survival did not depend on the method which was used to assess response. Considering the absolute figures of the median survival of these patients, the results were equal.

In conclusion, CT is far superior in picking out measurable disease than chest radiograph. However, in the subset of patients with measurable lesions, the chest radiograph is a usefull tool to assess response, particularly in case of peripherally localized tumors without atelectasis. In this subset of patients, the results are similar enough to warrant the use of chest radiograph as a method to evaluate response saving some inconvenience for the patient. Furthermore, in an era of cost-consciousness, it is important to realize that despite its imperfections the chest radiograph is an adequate tool in a selected group of patients. 


\section{References}

1. Brindle J.S, Shaw EG, Su JQ, et al. Pilot study of accelerated hyperfractionated thoracic radiation therapy in Stage III non-small cell lung carcinoma. Cancer 1993; 72: 405-409.

2. Dillman $\mathrm{RO}$, Seagren SL, Propert $\mathrm{KJ}$, et al. A randomized trial of induction chemotherapy plus high-dose radiation alone in Stage III non-small-cell lung cancer. N Eng J Med 1990; 323: 940-945.

3. Herskovic $\mathrm{A}$, Orton $\mathrm{C}$, Seyedsadr $\mathrm{M}$, et al. Initial experience with a practical hyperfractionated accelerated radiotherapy regimen. Int J Radiat Oncol Biol Phys 1991; 21: 1275-1281.

4. Saunders $M$ and Dische $S$. Continuous, hyperfractionated accelerated radiotherapy (CHART) in non-small cell carcinoma of the bronchus. Int J Radiat Oncol Phys 1990; 19: 1211-1215.

5. Soresi $E$, Clerici $E$, Grilli $R$, et al. A randomized clinical trial comparing radiation therapy versus radiation therapy plus cis-dichlorodiamin platinum (II) in the treatment of locally advanced non-small-cell lung cancer. Semin Oncol 1988; 15 (Suppl. 7): 20-25.

6. Trovo MG, Minatel E, Franchin G, et al. Radiotherapy versus radiotherapy enhanced by cisplatin in stage III non-small-cell lung cancer. Int J Radiat Oncol Phys 1992; 24: 11-15.

7. Yu E, Souhami. L, Guerra J, et al. Accelerated fractionation in inoperable non-small cell lung cancer. Cancer 1993; 71: 2727-2731.

8. UICC TNM Atlas. Illustrated guide to the TNM/PTNM-Classification of Malignant Tumours.Springer-Verlag 1997, Fourth Edition.

9. Altmann DG In Practical Statistics for Medical Research. London: Chapman \& Hall: 1992; 403-419.

10. Landis RJ and Koch CG. The measurement of observer agreement for categorical data. Biometrics 1977; 33: 159-174.

11. Lyn BE, Ayoub AW, Saunders MI and Dische S. Chest radiography or computed tomography to assess the response of lung cancer to radiotherapy. Clin Oncol R Coll Radiol 1992; 4: 154-159.

12. Dajczman $\mathrm{E}$, Hanley $\mathrm{J}$, Lisbona $\mathrm{A}$, et al. Comparison of response evaluation in small cell lung cancer using computerized tomography and chest radiography. Lung Cancer 1994; 11(1-2): 51-60.

13. Onitsuka $H, T$ sukada $M$, Araki $A$, et al. Differentation of central lung tumor from postobstructive lobar collapse by rapid sequence computed tomography. $J$ Thorac Imaging 1991; 6(2): 28-31.

14. Grover FL. The role of CT and MRI in staging of the mediastinum. Chest 1994; 106 (6 Suppl): 391S-396S.

15. Shioya $S$, Haida $M$, Ono $Y$, et al. Lung cancer: differentiation of tumor, necrosis and atelectasis by means of $\mathrm{T} 1$ and $T 2$ values measured in vivo. Radiology 1988; 167: 105-109.

16. Tobler J, Levitt RG, Glazer HS, et al. Differentiation of proximal bronchogenic carcinoma from postobstructive lobar collapse by Magnetic Resonance Imaging: comparison with computed tomography. Invest Radiol 1987;

22: $538-543$.

17. Herold CJ, KuhIman JE and Zerhouni E.A. Pulmonary atelectasis: signal patterns with MR Imaging. Radiology 1991; 178: 715-720.

18. Perez CA, Stanley $K$, Rubin $P$, et al. Patterns of tumor recurrence after definitive irradiation for inoperable nonoat cell carcinoma of the lung. Int J Radiat Oncol Biol Phys 1980; 6: 987-994.

19. Saunders MI, Barltrop MA, Rassa PM, et al. The relationship between tumor response and survival following radiotherapy for carcinoma of the bronchus. Int J Radiat Oncol Biol Phys 1984; 10: 499-501.

20. Sealy R, Lagakos S, Barkley T, et al. Radiotherapy of regional epidermoid carcinoma of the lung. Cancer 1982; 49: $1338-1345$. 
Chapter 6

\title{
Quality of life after palliative radiotherapy in non-small cell lung cancer: a prospective study.
}

\author{
Hans Langendijk \\ Guul ten Velde \\ Neil Aaronson \\ Jos de Jong \\ Martin Muller \\ Miel Wouters
}

Published in:

International Journal of radiation Oncology Biology and Physics 2000; 47: 149-155 


\section{Abstract}

Purpose: The purpose of this study was to investigate changes in respiratory symptoms and quality of life (QOL) in patients with locally advanced and metastatic non-small cell lung cancer (NSCLC) receiving thoracic radiotherapy. Additionally, the correlation between the level of symptom relief and objective tumour response was investigated.

Material and methods: Sixty-five patients were entered in this prospective study. The EORTC QLQ-C30 and EORTC QLQ-LC13 were used to investigate changes in QoL. Assessments were performed before radiotherapy and 2 weeks, 6 weeks and 3 months after radiotherapy.

Results: The QoL response rates were excellent for haemoptysis $(79 \%)$, good for arm/shoulder pain (56\%), chest wall pain (53\%) and cough (49\%), moderate for dyspnea $(39 \%)$ and minimal for the general symptoms fatigue (22\%) and appetite loss $(11 \%)$. The QoL response rates for the five functioning scales of the QLQ-C30 varied from 35\% for role functioning to $57 \%$ for emotional functioning. Global QoL improved in $37 \%$ of the cases. In general, there was a tendency for better palliation of symptoms and improvement of QoL among patients with an objective tumor response than among those without objective tumour response, which was statistically significant for dyspnea $(p=0.02)$ and social functioning $(\mathrm{p}=0.04)$.

Conclusions: This study confirms that conventional thoracic radiotherapy offers palliation of respiratory symptoms and improved QoL in a substantial proportion of patients with locally advanced and metastatic NSCLC. Tumor reduction is only one of the mechanisms by which palliation of symptoms and improvement of QoL is achieved. 


\section{Introduction}

Today, there is general agreement that quality of life (QoL) is a multidimensional concept concerned with the impact of disease and its treatment on physical, psychological and social well being, and that the emphasis should be placed on assessing the subjective experience of the person whose QoL is in question 1. A wide range of self-report instruments has been developed to assess quality of life in cancer patients, in general ${ }^{2-7}$. Some questionnaires have been validated for lung cancer populations while others have been developed and validated to assess respiratory symptoms ${ }^{8-11}$.

Many patients with advanced locoregional or metastatic non-small cell lung cancer will, at some point, have respiratory symptoms requiring treatment. Taking into account that many patients with advanced NSCLC are beyond cure, it becomes increasingly important to investigate the effects of treatment on patients' QoL. In a number of studies, radiotherapy has been shown to be an effective treatment modality in the palliation of respiratory symptoms due to an intrathoracic tumor ${ }^{12-15}$. However, until recently, little attention has been paid to assessing patients' self-rated QoL in palliative lung cancer trials.

This prospective study was initiated to investigate changes in respiratory symptoms and QoL using the EORTC questionnaires (QLQ-C30 and QLQ-LC13) in a group of patients with NSCLC with poor prognostic features treated by external irradiation. Palliation of respiratory symptoms and improvement or preservation of QoL was the main goal of treatment in these patients.

Based on the results of earlier studies ${ }^{13,14}$, our first hypothesis was that radiotherapy would offer excellent palliation (i.e. in $>75 \%$ of the cases) of haemoptysis and good palliation (i.e. in $>50 \%$ of the cases) of pain, cough and dyspnea. Furthermore, we expected a reduction of general symptoms in more than $50 \%$ of the cases and an improvement of different aspects of QoL in the majority of patients.

Although the exact mechanism by which palliation of respiratory symptoms is achieved by radiotherapy is not well established, it has been generally assumed that the degree of reduction in tumor size correlates with the level of symptom relief. Based on this assumption, our second hypothesis was that the degree of palliation of symptoms and improvement in QoL achieved in those cases with an objective tumor response should be 
higher compared to the degree of improvement observed in those cases without an objective tumor response.

\section{Material and methods}

\section{Patient eligibility}

From June 1994 to December 1996, 302 patients with inoperable NSCLC were referred to the Radiotherapeutisch Instituut Limburg (RTIL) for radiotherapy. Of these patients, 71 patients with a WHO performance status of 3 and/or supraclavicular lymph node metastases and/or distant metastases with symptoms related to intrathoracic tumor, were selected for palliative irradiation. For the present study, patients treated with surgery, chemotherapy and/or previous radiotherapy of the intrathoracic tumor were excluded, as

Table 1: Pre-treatment characteristics

\begin{tabular}{llcc}
\hline Variables & & $\mathrm{n}$ & $\%$ \\
\hline Stage (UICC 1992) & Stage I & 2 & 3 \\
& Stage II & 0 & 0 \\
& Stage IIIa & 9 & 14 \\
& Stage IIIb & 37 & 57 \\
& Stage IV & 17 & 26 \\
Tumour type & Squamous cell & 32 & 49 \\
& Adeno & 12 & 19 \\
Weight loss & Undifferentiated & 21 & 32 \\
& none & 21 & 33 \\
& 1-10\% & 19 & 30 \\
& $>10 \%$ & 24 & 38 \\
Performance status & unknown & 1 & \\
& WHO 0 & 6 & 9 \\
& WHO 1 & 21 & 32 \\
& WHO 2 & 16 & 25 \\
& WHO 3 & 22 & 34 \\
\hline
\end{tabular}


well as patients with brain metastases and patients irradiated in the same period on sites other than the intrathoracic tumour. After adjustment for these criteria, 69 patients were eligible and were asked to participate in the study. Two patients declined participation and 2 patients, who gave their informed consent, did not return their pre-treatment questionnaire before the start of radiotherapy. The pre-treatment characteristics of the remaining 65 patients who formed the final sample for this study are described in Table 1.

Fifty-nine patients $(91 \%)$ were male. The mean age at diagnosis was 65 years, ranging from 39 to 88 years. All patients were prospectively staged according to the guidelines of the UICC $1992{ }^{16}$. The staging procedure consisted of a physical examination, posteroanterior and lateral chest radiography, CT scan of the chest including the liver and adrenal glands, and bronchoscopy in all patients. Bone scintigraphy was performed only when indicated. Histologic or cytological confirmation of the diagnosis NSCLC was available for all patients.

\section{Quality of life assessment}

The EORTC QLQ-C30 (version 1.0) was used to assess QoL ${ }^{2}$. This questionnaire is composed of 30 questions organized into five functional scales (physical, role, cognitive, emotional and social), three symptom scales (fatigue, pain and nausea and vomiting), a global health/quality of life scale, and a number of single items assessing additional symptoms (dyspnea, sleep disturbance, constipation and diarrhea). For the assessment of respiratory symptoms, the EORTC lung cancer module (QLQ-LC13) was used. This supplemental questionnaire contains 13 questions concerning symptoms frequently present in lung cancer patients ${ }^{8}$. For the majority of functioning scales and symptom scales a 4point response scale is used, except for the physical and role functioning scales, where dichotomous response choices are employed, and for global health status/quality of life, where a 7-point scale is used. All scores are linearly converted to a 0 to 100 scale. For the functional and global health status/quality of life scales, higher scores represent a better level of functioning. For the symptom scales, higher scores represent a greater degree of symptoms. The reliability and validity of these questionnaires have been confirmed in international studies ${ }^{2,8}$. Quality of life and respiratory symptoms were assessed before the 
start of radiotherapy and subsequently 2 weeks, 6 weeks and 3 months after the end of radiotherapy.

Patients were asked to participate in the study during the first visit at the radiotherapy department. After informed consent, the questionnaire was distributed to the patients who were asked to return the questionnaire within 3 days by mail. During follow up, the questionnaires were send to the patients by mail. When the questionnaire was not returned within 4 days, the patient was telephoned and asked to complete and return the questionnaire.

\section{Radiotherapy}

The target volume included the primary tumor and enlarged mediastinal and supraclavicular lymph nodes with a margin of 2 centimeters, as well as the mediastinal lymph node areas on both sides. The target volume was irradiated with $3 \mathrm{~Gy}$ per fraction (4 times a week) up to a total dose of $30 \mathrm{~Gy}(100 \%)$. In all patients, two opposing anterior-posterior/posterior-anterior fields were used. No correction for lung tissue density was made. For the coverage of normal lung tissue standard or individual lead blocking was used.

\section{Objective tumor response}

To assess objective tumor response the product of the 2 largest diameters measured was calculated before and 2 to 6 weeks after radiotherapy using chest radiography and/or CT scan of the chest ${ }^{17}$. A response was defined as a complete disappearance or as a reduction of $50 \%$ or more of the product of the 2 largest diameters. A reduction of less than $50 \%$ or progression was classified as non-response. A bronchoscopy to evaluate tumor response was not routinely performed.

StatisticsThe proportion of patients showing a response in symptoms and QoL was calculated. To assess the response rate for symptoms, each symptom scale was first divided into four categories. For the single-item scales (cough, haemoptysis, pain arm/shoulder, pain chest wall and appetite loss), four ranges of scores were defined: $1=$ nil, $2=$ mild, $3=$ moderate and $4=$ severe. For the multi-item scales (dyspnea and fatigue), four 
Table 2: Response criteria for general and respiratory symptoms.

\begin{tabular}{|c|c|c|c|}
\hline \multirow[t]{2}{*}{ Follow-up } & \multicolumn{3}{|c|}{ Baseline score } \\
\hline & Moderate or severe & Mild & Nil \\
\hline $\begin{array}{l}\text { Mild on two consecutive } \\
\text { assessments }\end{array}$ & Improvement & Control & No response \\
\hline Nil on two consecutive assessments & Improvement & Improvement & Prevention \\
\hline Dead before first assessment & $\begin{array}{l}\text { Dead without } \\
\text { palliation }\end{array}$ & $\begin{array}{l}\text { Dead without } \\
\text { palliation }\end{array}$ & Not evaluable \\
\hline $\begin{array}{l}\text { Dead before second assessment } \\
\text { with mild on first assessment }\end{array}$ & Not evaluable & $\begin{array}{l}\text { Dead without } \\
\text { palliation }\end{array}$ & $\begin{array}{l}\text { Dead without } \\
\text { palliation }\end{array}$ \\
\hline $\begin{array}{l}\text { Dead before second assessment } \\
\text { with nil on first assessment }\end{array}$ & Not evaluable & Not evaluable & Not evaluable \\
\hline
\end{tabular}

ranges of scores were defined as well, based on the converted score ranging from 0 to 100 : $0=$ nil, 1 to $34=$ mild, 35 to $67=$ moderate and 68 to $100=$ severe .

A QoL response rate is often calculated over the subset of patients having a specific symptom at baseline and follow-up assessment. When the follow-up score for a symptom is lower than that reported at baseline, the patient is considered palliated. Conversely, when the score is higher, the patient is considered to have deteriorated. We chose to employ an alternative definition of palliation, derived from the proposal of Stephens and co-workers ${ }^{18}$, which also takes into account patients with no or mild symptoms at baseline and those who died before the first follow-up assessment. Specifically, the following criteria were used to define palliation of symptoms (Table 2): patients with a moderate or severe symptom at baseline, who reported a reduction to nil or mild on two consecutive assessments in the first 3 months after therapy, were classified as responders (improvement); patients with a mild symptom at baseline, who reported a reduction to nil on two consecutive assessments in the first 3 months after therapy, were classified as responders as well (improvement); those with a mild symptom at baseline which did not worsen at follow-up (control) and those with no symptoms at baseline without deterioration during follow-up (prevention), were also classified as responder; patients with a mild, moderate or severe symptom at baseline who died before the first follow-up assessment were classified as non-responders (dead without palliation); patients with a mild, moderate or severe symptom at baseline without 
Table 3: Response criteria for functioning scales and global quality of life.

\begin{tabular}{|c|c|c|c|}
\hline \multirow[t]{2}{*}{ Follow-up } & \multicolumn{3}{|c|}{ Baseline score } \\
\hline & $0-59$ & $60-79$ & $80-100$ \\
\hline $\begin{array}{l}\text { Increase of at least } 5 \text { points on two } \\
\text { consecutive assessments to a minimal value } \\
\text { of } 40\end{array}$ & Improvement & Improvement & Improvement \\
\hline $\begin{array}{l}\text { No change (i.e. }<5 \text { points) on two } \\
\text { consecutive assessments }\end{array}$ & No change & Control & Prevention \\
\hline Dead before first assessment & $\begin{array}{l}\text { Dead without } \\
\text { palliation }\end{array}$ & $\begin{array}{l}\text { Dead without } \\
\text { palliation }\end{array}$ & $\begin{array}{l}\text { Dead without } \\
\text { palliation }\end{array}$ \\
\hline $\begin{array}{l}\text { Dead before second assessment without an } \\
\text { increase of at least } 5 \text { points to a minimal } \\
\text { vlaue of } 40 \text { on first assessment }\end{array}$ & $\begin{array}{l}\text { Dead without } \\
\text { palliation }\end{array}$ & $\begin{array}{l}\text { Dead without } \\
\text { palliation }\end{array}$ & $\begin{array}{l}\text { Dead without } \\
\text { palliation }\end{array}$ \\
\hline $\begin{array}{l}\text { Dead before second assessment with an } \\
\text { increase of at least } 5 \text { points to a minimal } \\
\text { vlaue of } 40 \text { on first assessment }\end{array}$ & Not evaluable & Not evaluable & Not evaluable \\
\hline
\end{tabular}

Note 1: patients were classified as responders in case of improvement, prevention or control

Note 2: patients were classified as non-responders in case of no change or dead without palliation

improvement to at least mild at first follow-up and who died before the second follow-up assessment were also classified as non-responders (dead without palliation). Patients without symptoms at baseline who died before the first follow-up assessment or who died before the second follow-up assessment without deterioration were considered to have inadequate follow-up data.

Osoba and co-workers investigated the significance of changes in health-related QoL scores assessed with the QLQ-C30 by means of a subjective significance questionnaire (SSQ), in which patients were posed direct questions about perceived changes in physical, emotional and social functioning and in global QoL ${ }^{19}$. For patients who indicated "no change" in the SSQ, the mean scores in the corresponding QLQ-C30 domains was not significantly different from 0 . For patients who indicated "a little" change either for better or worse, the mean change in scores was about 5 to 10 points on a 100-point scale. In the present study, a response classification system for the functioning scales and global QoL scale was used (Table 3), derived from Stephens ${ }^{18}$ and Osoba ${ }^{19}$. Patients with a baseline score ranging from 0 to 39 who showed an increase of at least 5 points on two consecutive follow-up assessments to a minimal value of 40 were classified as responders (improvement). Patients with a baseline score of 40 or higher who showed an increase from baseline of at least 5 points on two consecutive follow-up assessments were also classified 
as responders (improvement). Patients with a baseline value varying from 61 to 80 who maintained this score (control) and patients with a value varying from 81 to 100 who maintained this score on two consecutive assessments (prevention) were classified as responders as well.

To analyze the association between objective tumor response and changes in QoL we compared the change scores for QoL and symptoms between the objective responders and objective non-responders by means of repeated measures analysis of variance (ANOVA). Results were regarded as statistically significant if the probability of chance was $5 \%$ or less.

\section{口 Results}

\section{Compliance}

Of the 67 patients who gave their informed consent at the beginning of the study, 65 returned the questionnaire before radiotherapy ( $97 \%$ ). The compliance was $91 \%$ (52 of 57 patients alive) at 2 weeks and $91 \%$ ( 40 of 44 patients alive) at 6 weeks post-radiotherapy. At 3 months the compliance was somewhat lower, i.e. $79 \%$ (26 of 33 patients alive). In most cases, patients did not return the questionnaire because they were in the terminal phase of their illness.

\section{Pre-treatment symptoms}

The proportion of patients presenting with specific symptoms on admission for the domains assessed with the QLQ-C30 and QLQ-LC13 are presented in table 4. Fatigue $(94 \%)$, pain $(86 \%)$ and appetite loss $(71 \%)$ were the most frequently reported general symptoms and cough $(89 \%)$, dyspnea $(88 \%)$ and pain in the chest wall $(62 \%)$ were the most frequently reported respiratory symptoms. The mean symptom scores in all patients varied from 14.4, for dysphagia and nausea and vomiting to 54.4 for fatigue. The mean score for all patients was 43.6 , for physical functioning, 50.0 , for role functioning, 56.7 , for emotional functioning, 73.1 , for emotional functioning, 69.7 , for social functioning and 40.1, for global QoL. 
Table 4: Pre-treatment symptoms.

\begin{tabular}{lcc}
\hline Symptom or functioning scale & \% of patients & $\begin{array}{c}\text { Mean score in } \\
\text { all patients } \\
\text { (SD) }\end{array}$ \\
& & \\
\hline Symptoms & & \\
Fatigue & 94 & $54.4(26.8)$ \\
Cough & 89 & $51.3(28.9)$ \\
Dyspnea & 88 & $46.3(30.8)$ \\
Pain & 86 & $42.3(32.4)$ \\
Appetite loss & 71 & $47.7(36.9)$ \\
Pain chest wall & 62 & $34.9(33.6)$ \\
Insomnia & 57 & $35.4(36.7)$ \\
Haemoptysis & 46 & $21.5(26.6)$ \\
Pain arm/shoulder & 43 & $28.2(36.9)$ \\
Nausea and vomiting & 34 & $13.8(26.3)$ \\
Constipation & 31 & $17.4(31.2)$ \\
Dysphagia & 25 & $14.4(27.6)$ \\
Functioning scales \& global quality of life & & \\
Physical functioning & - & $69.7(34.0)$ \\
Role functioning & - & $40.1(22.3)$ \\
Emotional functioning & - & $53.6(32.9)$ \\
Cognitive functioning & $-0(37.5)$ \\
Social functioning & - & $56.7(23.8)$ \\
Global quality of life & $-127.0)$ \\
\hline & - & \\
\hline
\end{tabular}

\section{Symptom and quality of life response}

Of the general symptoms assessed with the QLQ-C30, only those considered to be the most relevant in this category of patients were taken into account. The response rates for fatigue and appetite loss were $21 \%$ and $33 \%$, respectively (Table 5). The response rates for the respiratory symptoms varied from $36 \%$ for dyspnea to $79 \%$ for haemoptysis (Table $5)$. 
Table 5: Response classification for general and respiratory symptoms $(n=65)$.

\begin{tabular}{lcrrrrr}
\hline Symptom & $\begin{array}{c}\text { Inadequate } \\
\text { follow up } \\
\text { data }\end{array}$ & Improvement & Control & Prevention & \multicolumn{1}{c}{$\begin{array}{c}\text { Dead without } \\
\text { palliation }\end{array}$} & Response rate \\
\hline Cough & 13 & $14 / 45(31 \%)$ & $8 / 21(38 \%)$ & $3 / 7(14 \%)$ & $12 / 52(23 \%)$ & $25 / 52(48 \%)$ \\
Haemoptysis & 18 & $11 / 26(42 \%)$ & $5 / 18(28 \%)$ & $20 / 21(95 \%)$ & $4 / 47(9 \%)$ & $37 / 47(79 \%)$ \\
Dyspneu & 12 & $15 / 45(33 \%)$ & $7 / 14(50 \%)$ & $3 / 8(38 \%)$ & $13 / 53(25 \%)$ & $19 / 53(36 \%)$ \\
Pain arm/shoulder & 19 & $7 / 21(33 \%)$ & $2 / 5(40 \%)$ & $17 / 25(68 \%)$ & $6 / 46(13 \%)$ & $26 / 46(57 \%)$ \\
Chest pain & 18 & $12 / 32(38 \%)$ & $5 / 15(33 \%)$ & $11 / 15(73 \%)$ & $7 / 47(15 \%)$ & $28 / 47(60 \%)$ \\
Fatigue & 13 & $5 / 58(9 \%)$ & $4 / 10(40 \%)$ & $2 / 4(50 \%)$ & $12 / 48(25 \%)$ & $11 / 52(21 \%)$ \\
Appetite loss & 17 & $8 / 37(22 \%)$ & $1 / 9(11 \%)$ & $7 / 12(58 \%)$ & $8 / 48(17 \%)$ & $16 / 48(33 \%)$ \\
\hline
\end{tabular}

* Note: the number of patients with inadequate follow up data may differ between scales because of the definition of 'inadequate follow up' (see also table 2)

Table 6: Response classification for functioning and global quality of life $(n=65)$.

\begin{tabular}{lcccccc}
\hline Symptom & $\begin{array}{c}\text { Inadequate } \\
\text { follow up } \\
\text { data }\end{array}$ & Improvement & Control & Prevention & $\begin{array}{c}\text { Dead without } \\
\text { palliation }\end{array}$ & Response rate \\
\hline Physical functioning & 11 & $14 / 48(29 \%)$ & $2 / 13(15 \%)$ & $3 / 6(50 \%)$ & $14 / 54(26 \%)$ & $19 / 54(35 \%)$ \\
Role functioning & 17 & $8 / 35(23 \%)$ & no patients & $9 / 13(69 \%)$ & $8 / 48(17 \%)$ & $17 / 48(35 \%)$ \\
Emotional functioning & 19 & $16 / 34(47 \%)$ & $1 / 11(9 \%)$ & $6 / 12(50 \%)$ & $5 / 46(11 \%)$ & $26 / 46(57 \%)$ \\
Cognitive functioning & 17 & $7 / 24(29 \%)$ & $0 / 12(0 \%)$ & $14 / 24(58 \%)$ & $8 / 48(17 \%)$ & $22 / 48(46 \%)$ \\
Social functioning & 20 & $4 / 19(21 \%)$ & $1 / 4(25 \%)$ & $12 / 26(46 \%)$ & $1 / 45(2 \%)$ & $19 / 45(42 \%)$ \\
Global QoL & 18 & $18 / 45(40 \%)$ & $1 / 6(17 \%)$ & $0 / 2(50 \%)$ & $7 / 47(15 \%)$ & $19 / 47(40 \%)$ \\
\hline
\end{tabular}

* Note: the number of patients with inadequate follow up data may differ between scales because of the definition of 'inadequate follow up' (see also table 2).

The response classification for the functioning scales and global QoL scale are summarized in Table 6 . The response rates varied from $57 \%$ for emotional functioning to $35 \%$ for role functioning.

Relation between objective tumor response and changes in respiratory symptoms and quality of life

Information on objective tumor response was available for 32 of the 40 patients who were still at risk after 6 weeks. Eight 8 patients (25\%) were classified as having had an objective tumor response. Responders reported significantly greater improvement over time in dyspnea and fatigue compared to the non-responders. Although not statistically significant, a trend towards better palliation of haemoptysis was observed among 
responders than non-responders (Figure 1). No statistically significant differences between objective responders and non-responders were observed in the degree of improvement on the functioning scales and global QoL scales (Figure 2).

\section{Discussion}

The present study is one of the first to investigate changes in respiratory symptoms and quality of life using standardized QoL questionnaires in a typical population of patients with locally advanced and/or metastatic NSCLC treated with conventional palliative radiotherapy $(10 \times 3 \mathrm{~Gy})$. Before starting the study, some doubts were expressed with regard to the expected compliance in these patients who generally had a poor performance status. Other groups had reported difficulties in collecting quality of life data in patients with poor performance status or advanced disease ${ }^{20-23}$. By appointing a specific individual (other than the treating physicians) to collect the QoL data, and by limiting the frequency with which questionnaires were administered, we were able to achieve a compliance of approximately $90 \%$. In general, our impression was that patients were very motivated and willing to fill in the questionnaires, even in case of deteriorating general condition.

The present study has documented excellent palliation of haemoptysis $(79 \%)$ and good palliation of pain in the arm/shoulder (52\%), chest pain $(60 \%)$ and cough (48\%). Palliation of dyspnea (36\%) was less satisfactory. Most of these response rates are similar to those reported by the MRC lung cancer working party ${ }^{13}, 14$. In the first MRC randomized trial patients were allocated to receive two fractions of 8.5 Gy 1 week apart or a multifractionated schedule of either $30 \mathrm{~Gy}$ in ten fractions or $27 \mathrm{~Gy}$ in six fractions. With the multifractionated schedule, palliation as assessed by the clinicians was achieved in $56 \%$ of cases for cough, $86 \%$ for haemoptysis, $80 \%$ for chest pain and $57 \%$ for breathlessness. For the two fraction regimen, comparable results were observed. In the second MRC trial comparable results were also found after a single fraction of $10 \mathrm{~Gy}{ }^{14}$. The two MRC studies appeared to yield higher rates of palliation than our results for chest pain $(80 \%$ versus $60 \%)$ and dyspnea $(57 \%$ versus $36 \%)$. There are several possible explanations for these differences. 
Figure 1: Change scores respiratory and general symptoms stratified by objective tumour response.
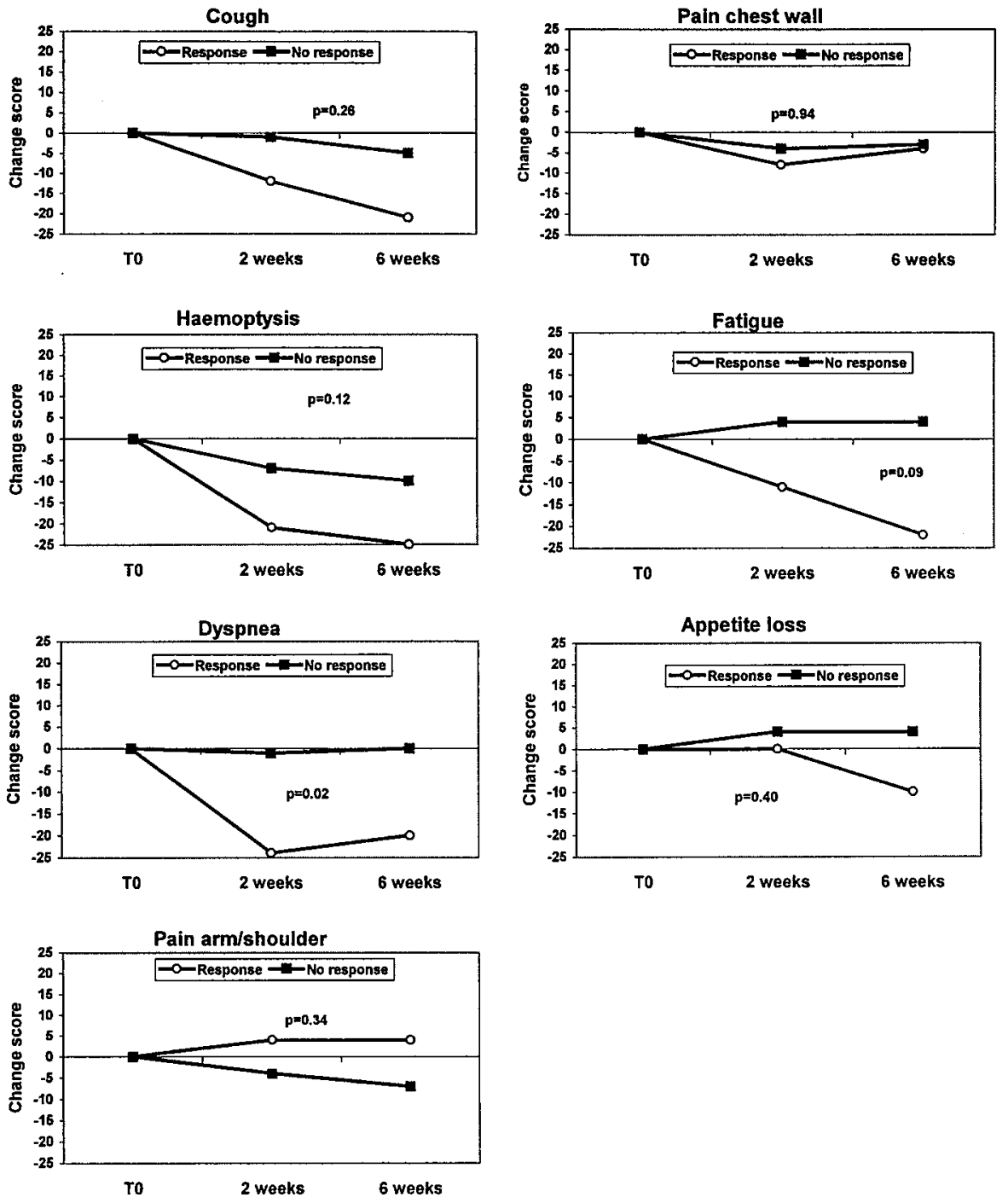

First, there may be a difference in study samples, i.e. trial based with possible selection bias versus non-trial based. Secondly, the source of symptom rating (physician versus patient) was different between the two studies. Thirdly, other authors already pointed out 
Figure 2: Change scores for functioning scales and global QoL stratified by objective tumour response.
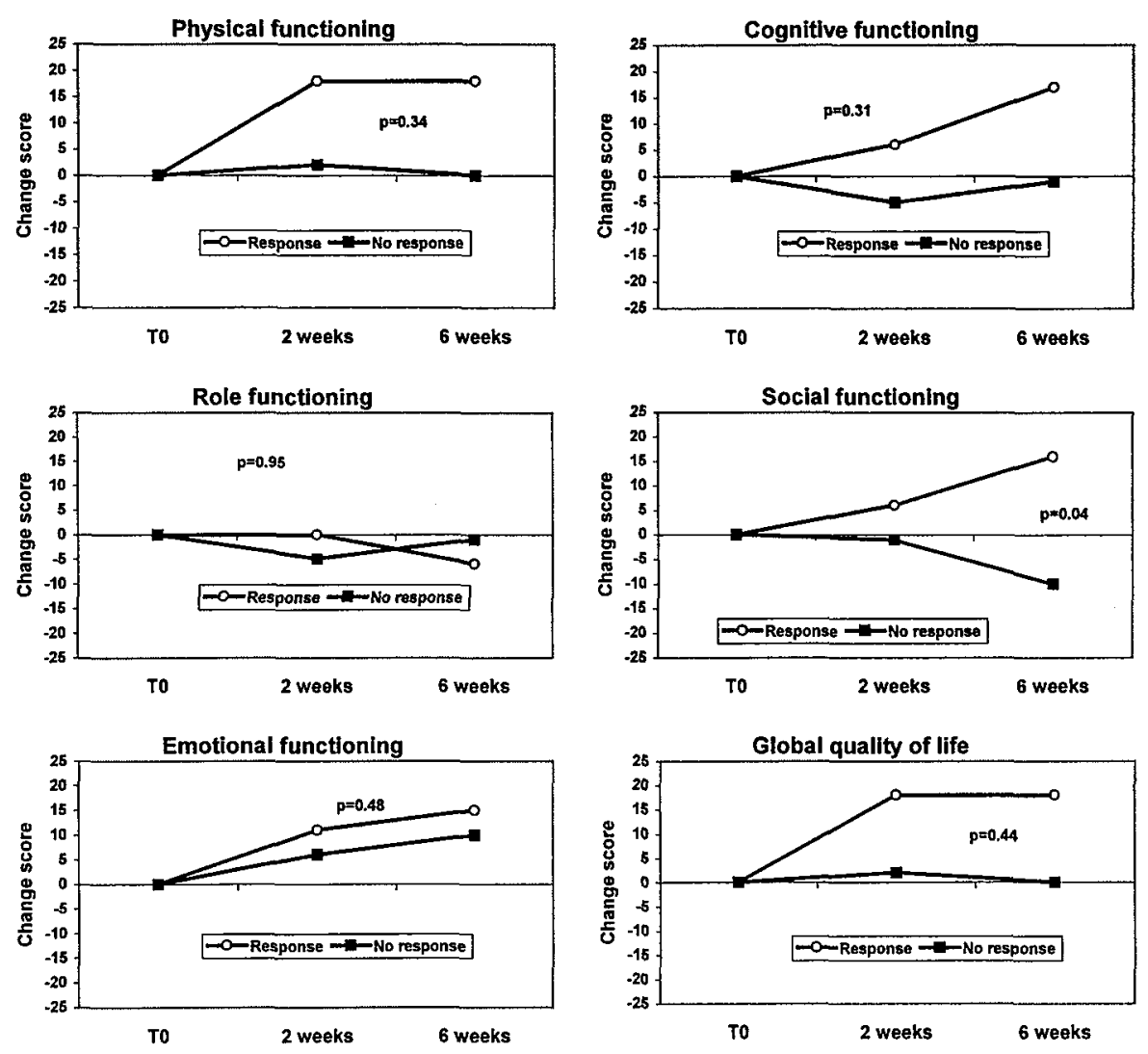

that different QoL instruments can not be replaced by the others and that direct comparison of results obtained with different instruments is not possible ${ }^{24,25}$.

Although short palliative fractionation schedules appeared to offer good results for respiratory symptoms, the question remained as to whether an improvement could also be achieved with regard to different aspects of functioning and global quality of life. In the present study, approximately half of the patients showed significant improvement for physical, emotional and cognitive functioning. Improvement in role functioning, social functioning and global QoL was observed in $35 \%$ to $42 \%$ of the cases. The latter response rates were somewhat lower than we had expected. This may be explained by the fact that 
improvement had to be observed on at least two consecutive assessments, and because patients who died without palliation before the first or second assessment after radiotherapy were classified as non QoL-responder.

In the group of patients with an objective tumor response, better palliation of dyspnea was observed. Additionally, the change score for social functioning was significantly better among the patients with an objective tumor response than in those without objective tumor response. No significant differences between the responders and non-responders were observed with regard to changes in the other functioning scales or the global QoL scale. However, this may be due to the relatively small number of patients available for these latter analyses, as the change scores among the responders appeared to be better, although not statistically significant, than the scores among the non-responders for almost all the scales. Pujol and co-workers reported on 54 patients with NSCLC treated with chemotherapy. In their study improved or stable quality of life was highly correlated with tumor response ${ }^{26}$. These results support the hypothesis that tumor reduction is one of the mechanisms by which palliation of some symptoms is achieved. However, palliation of pain in the arm/shoulder and pain in the chest were not associated significantly with objective tumor response. Possibly, palliation of pain in this category of patients is not achieved by the mechanism of tumor reduction but by other mechanisms, such as inhibition of intracellular prostaglandin synthesis ${ }^{27}$.

In conclusion, conventional thoracic radiotherapy offers palliation of respiratory symptoms and improvement in QoL, in a substantial proportion of patients with locally advanced and metastatic NSCLC. Tumor reduction is an important, but not the only mechanism by which palliation of most symptoms and improvement of quality of life is achieved. 


\section{References}

1. Aaronson, NK. Methodological issues in assessing the quality of life of cancer patients. Cancer 1991; 67 (suppl): 839-850.

2. Aaronson, NK, Ahmedzai S, Bergman, B, et al. The European Organization for Research and Treatment of Cancer QLQ-C30: a quality of life instrument for use in international clinical trials in oncology. J Natl Cancer Inst 1993; 85: 365-76.

3. Fayers PM, Bleehen NM, Girling DJ and Stephens R. Assessment of quality of life in small cell lung cancer using a daily diary card developed by the Medical Research Council Working Party. Br J Cancer 1991; 64: 299-306.

4. Schag CA, Ganz PA and Heinrich RL. Cancer, Rehabilitation Evaluation System-short form (CARES-SF). A cancer specific rehabilitation and quality of life instrument. Cancer 1991; 68(6): 1406-13.

5. Schipper $\mathrm{H}$, Clinch J, McMurray $A_{1}$ et al. Measuring quality of life of cancer patients: The Functional Living IndexCancer: Development and validation. J Clin Oncol 1984; 2: 472-483.

6. Spitzer WO, Dobson AJ, Hall $\mathrm{J}$, et al. Measuring the quality of life of cancer patients. Chron Dis 1981; 34: 585-97.

7. Te Velde A, Sprangers MA and Aaronson NK. Feasibility, psychometric performance, and stability across modes of administration of the CARES-SF. Ann Oncol 1996; 7(4): 381-90.

8. Bergman B, Aaronson NK, Ahmedzai S, et al. The EORTC QLQ-LC13: a modular supplement to the EORTC Core Quality of Life Questionnaire (QLQ-C30) for use in lung cancer clinical trials in oncology. Eur J Cancer 1994; 30A: 635-42.

9. Cella DF, Tulsky DS, Gray G, et al. The functional Assessment of Cancer Therapy Scale: Development and validation of the general measure. J Clin Oncol 1993; 11: 570-9.

10. Cella DF, Bonomi AE, Lloyd SR, et al. Reliability and validity of the Functional Assessment of Cancer Therapy-Lung (FACT-L) quality of life instrument. Lung Cancer 1995; 12: 199-220.

11. Hollen PJ, Gralla RJ, Kris MG, et al. Quality of life assessment in individuals with lung cancer: Testing the Lung Cancer Symptom Scale (LCSS). Eur J Cancer 1993; 29A (suppl 1): S51-S58.

12. Collins TM, Ash DV, Close $\mathrm{HJ}$, et al. An evaluation of the palliative role of radiotherapy in inoperable carcinoma of the bronchus. Clin Radiol 1988; 39: 284-286.

13. Medical Research Council Lung Cancer Working Party. Inoperable non-small cell lung cancer (NSCLC): a Medical Research Counsel randomized trial of palliative radiotherapy with two or ten fractions. Br J Cancer 1991; 63: 26570.

14. Medical Research Council Lung Cancer Working Party. A Medical Research Counsel (MRC) randomized trials of palliative radiotherapy with two fractions or a single fraction in patients with inoperable non-small cell lung cancer (NSCLC) and poor performance status. Br J Cancer 1992; 65: 934-41.

15. Slawson RG and Scott RM. Radiation therapy in bronchogenic carcinoma. Radiology 1979; 132: 175-76; 1979.

16. In Hermanek P and Sobin LH (Ed) In: TNM Atlas: Classification of malignant tumours; Springer-Verlag, 75-84; 1992.

17. Langendijk JA, Lamers RJS, ten Velde GPM, et al. Is the chest radiograph a reliable tool in the assessment of tumor response after radiotherapy in nonsmall cell lung carcinoma. Int $J$ Radiation Oncology Biol Phys 1998; 41: 1037-1045.

18. Stephens RJ, Hopwood $P$ and Girling DJ. Defining and analysing symptom palliation in cancer clinical trials: a deceptively difficult exercise. $\mathrm{Br} J$ Cancer 1999; 79: 538-544.

19. Osoba $\mathrm{D}$, Rodrigues $\mathrm{G}$, Myles $\mathrm{J}$, et al. Interpreting the significance of changes in quality of life scores. $\mathrm{J}$ Clin Oncol 1998; 16: 139-44. 
20. Ganz PA, Haskell GM, Figlin RA, et al.. Estimating the quality of life in a clinical trial of patients with metastatic lung cancer using the Karnofsky performance status and the Functional Living Index-Cancer. Cancer 1988; 61: 849-56.

21. Ganz PA, Figlin RA, Haskell CM, et al. Supportive care versus supportive care and combination chemotherapy in metastatic non-small cell lung cancer. Does chemotherapy make a difference? Cancer 1989; 63: 1271-178.

22. Geddes DM, Dones L, Hill E, et al. Quality of life during chemotherapy for small cell lung cancer: assessment and use of daily diary card in a randomized trial. Eur $J$ Cancer 1990; 26: 484-92.

23. Giaccone G, Splinter TA, Debruyne $C$, et al. Randomized study of paclitaxel-cisplatin versus cisplatin-teniposide in patients with advanced non-small cell lung cancer. The European Organization for Research and Treatment of Cancer Lung Cancer Cooperative Group. J Clin Oncol 1998; 16(6): 2133-41.

24. Kemmler G, Holzner $B, K o p p, M$, et al. Comparison of two quality-of-life instruments for cancer patients: The Functional Assessment of Cancer Therapy-General and the European Organization for research and Treatment of Cancer Quality of Life Questionnaire-C30. J Clin Oncol 1999; 17: 2932-2940.

25. Hollen $P$ and Gralla JRJ. Comparison of instruments for measuring quality of life in patients with lung cancer. Sem Oncol 1996; 23 (2, suppl 5): 31-40.

26. Pujol JL, Monnier A, Berille J, et al. Phase II study of nitrosourea fotemustine as single-drug chemotherapy in poorprognosis non-small-cell lung cancer. $\mathrm{Br} J$ Cancer $1994 ; 69(6)$ : 1136-40

27. Bates T. Radiotherapy for bone metastases. Clin Oncol (Royal College of Radiologists) 1989; 1 (2): 57-58. 


\title{
Chapter 7
}

\section{A prospective study on quality of life before and after radical radiotherapy in non-small cell lung cancer.}

\author{
Hans Langendijk \\ Neil Aaronson \\ Jos de Jong \\ Guul ten Velde \\ Martin Muller \\ Rob Lamers \\ Ben Slotman \\ Miel Wouters
}




\section{Abstract}

Purpose: The purpose of this study was to investigate changes in respiratory symptoms and quality of life (QoL) in patients with non-small cell lung cancer (NSCLC) receiving radical radiotherapy ( $60 \mathrm{~Gy}$ ). Additionally, the association between the level of symptom relief and objective tumor response, as well as with radiation-induced pulmonary changes was investigated.

Patients and methods: One hundred sixty-four patients were entered in this prospective study. The EORTC QLQ-C30 and EORTC QLQ-LC13 were used to investigate changes in QoL. Assessments were performed before radiotherapy and 2 weeks, 6 weeks and 3, 6, and 12 months after the completion of radiotherapy.

Results: The QoL response rates were excellent for haemoptysis (83\%), good for chest pain $(68 \%)$, arm/shoulder pain $(63 \%)$ and appetite loss $(59 \%)$ and poor for dyspnea $(37 \%)$, cough $(31 \%)$ and fatigue $(28 \%)$. The QoL response rates for the five functioning scales of the QLQ-C30 varied from $35 \%$ for physical functioning to $55 \%$ for social and cognitive functioning. Global QoL improved in $36 \%$ of the cases. A significant association was found between tumor response and palliation of chest pain, arm/shoulder pain, fatigue and physical functioning. During radiotherapy, a significant increase for most general symptoms and a deterioration in functioning and QoL was noted.

Conclusions: This study is the first to describe palliation and changes in QoL in radically irradiated patients with NSCLC. Radical radiotherapy offers palliation of respiratory symptoms and improved QoL in a substantial proportion of patients with NSCLC who have relatively good prognostic features. Although, tumor reduction is associated with palliation of respiratory symptoms, it can not serve as a surrogate for palliation. 


\section{Introduction}

Radiotherapy is an important treatment modality in patients with inoperable non-small cell lung carcinoma (NSCLC). Since a previous randomized study has shown a dose-response relationship for radiotherapy in NSCLC [1], many patients with locally advanced disease will receive radical radiotherapy (i.e., a total dose of $60 \mathrm{~Gy}$ or more) in order to achieve long-term loco-regional tumor control. Despite these radical doses, survival in these patients remains relatively poor, with median survival times in stage III varying from 8 to 12 months $^{1-4}$.

Given the relatively poor prognosis of inoperable NSCLC treated with radical radiotherapy, the inclusion of quality of life (QoL) as an endpoint of treatment becomes increasingly important. Although overall survival remains of primary interest, it should be interpreted in the light of symptom palliation and overall QoL because the side effects of radiotherapy may negate the putative benefit of improved survival. In recent years, there has been an increasing interest in evaluating the QoL of patients undergoing cancer treatment. This interest has led to the development of a wide range of self-report instruments to assess QoL ${ }^{5-13}$. Palliation of symptoms and QoL has been investigated in a number of studies of patients with lung cancer ${ }^{14-19}$. In almost all of these studies, only patients treated with palliative radiotherapy were included. Less information regarding QoL is available for those patients treated with radical or curative radiotherapy.

The aim of the present study was to evaluate changes in QoL and respiratory symptoms in patients with inoperable NSCLC selected for radical radiotherapy. Three specific hypotheses were investigated. Based on the results of an earlier study among patients with NSCLC treated with palliative radiotherapy ${ }^{20}$, we hypothesized that radical radiotherapy would offer excellent palliation (i.e., in $>75 \%$ of the cases) of haemoptysis and good palliation (i.e., in $>50 \%$ of the cases) of pain, cough and dyspnea. Additionally, we expected a reduction in general symptoms (e.g., fatigue and appetite loss) and an improvement in the range of QoL parameters in the majority of patients. Given the expected prolongation of loco-regional tumor control achieved with radical radiotherapy, we also expected that symptoms and QoL improved or remained stable for at least 6 months after the completion of radiotherapy. 
More specifically, the exact mechanism by which palliation of respiratory symptoms is achieved by radiotherapy is not well established. It is generally believed that the degree of reduction in tumor size correlates well with the level of symptom relief. Based on this assumption, our second hypothesis was that the degree of palliation of symptoms and improvement in QoL achieved in those cases with an objective tumor response should be significantly higher than observed in those cases without an objective tumor response.

Radiation-induced pulmonary changes occur frequently after high dose radiotherapy. Although, one of the aims of treatment is to palliate respiratory symptoms, these side effects may counterbalance the positive effects of radiotherapy. Therefore, our final hypothesis was that the degree of palliation of symptoms and improvement in QoL achieved in those cases without radiation-induced pulmonary changes should be higher than that observed in those cases in which radiation-induced pulmonary changes occurred.

\section{Material and methods}

\section{Patient eligibility and characteristics}

Between June, 1994 and December 1996, 174 patients were referred to our department for radical radiotherapy. Five patients $(3 \%)$ were excluded because the diagnosis could not be confirmed pathologically, 3 patients $(2 \%)$ because no baseline QoL assessment was returned and 2 patients ( $1 \%$ ) declined to participate in the study. The remaining 164 patients formed the sample for this study. All patients had a WHO-performance status 0 to 2, stage Illa or Illb disease (without supraclavicular lymph node metastases) according to the UICC $1992^{21}$, or stage I or II disease with a tumor diameter larger than 4 centimeters. Patients with supraclavicular lymph node metastases were excluded because a retrospective analysis of patients with NSCLC treated in our department indicated that these patients had such a short survival that a shorter fractionation schedule (10 $\times 3 \mathrm{~Gy})$ was considered more appropriate (unpublished data). For all patients, histologic and/or cytological confirmation of the diagnosis of NSCLC was available. Previously treated patients and/or patients who were treated with endobronchial brachytherapy or chemotherapy were excluded. In the period that the study was open for inclusion, the 
standard treatment approach for patients with inoperable NSCLC in the RTIL was radiotherapy alone. More than $90 \%$ of the patients treated with radical radiotherapy were included in the study. The large majority of patients $(84 \%)$ were male. The median age was 68 years, ranging from 37 to 84 years. There were 18 patients (11\%) with stage I disease, two patients with stage II disease (1\%), 64 patients with stage Illa disease (40\%) and 79 patients with stage Illb disease (48\%). All stage I patients were considered inoperable due to co-morbid disease, in particular, inadequate pulmonary function. Ninetyfour patients $(58 \%)$ had a squamous cell carcinoma, 28 patients $(17 \%)$ had an adenocarcinoma and 41 patients $(25 \%)$ had a large cell undifferentiated carcinoma. Sixtythree patients (38\%) had WHO-performance status 0,78 patients $(48 \%)$ had WHO 1 , and 23 patients (14\%) had WHO 2.

In 106 patients $(65 \%)$, comorbid disease related to smoking was present. There were 78 patients (48\%) with chronic obstructive lung disease, 29 patients (18\%) with coronary vascular disease, 24 patients (15\%) with hypertension, 24 patients (15\%) with peripheral vascular diseases, 21 patients $(13 \%)$ with congestive heart failure and 3 patients $(2 \%)$ who had a cerebrovascular accident in the medical history. In some patients more that one comorbid disease was present. Other comorbid diseases included, connective tissue disease (5 patients), diabetes (5 patients), chronic liver disease ( 1 patient) and renal failure (1 patient).

\section{Radiotherapy protocol}

The planning target volume of the initial field encompassed the primary tumor and enlarged lymph nodes with a margin of 2 centimeters, the ipsilateral hilus and the mediastinal lymph nodes on both sides. The target volume of the initial field was treated with fraction doses of 2.25 Gy (4 times per week) to a total dose of 45 Gy. Field arrangements were assessed by a localization/simulation procedure or by CT-assisted planning and individual blocks were used to shield normal tissue. In almost all cases, the initial field was treated using opposing anterior-posterior/posterior-anterior fields. The target volume of the boost included the nodes considered pathological and the primary site with a 1-centimeter margin. This target volume was treated with an additional $15 \mathrm{~Gy}$, using fraction-doses of $2.5 \mathrm{~Gy}$ (4 times a week) to a total dose of $60 \mathrm{~Gy}$. In the majority of cases, for the boost, a three- or four-field technique was used. The dose was specified in 
the isocenter and the target volumes described received at least $90 \%$ of the prescribed dose. In all cases, correction was made for lung tissue density (0.3).

All patients had optimal supportive care provided by a multidisciplinary team, including radiation oncologists, pulmonologists and pain experts, before, during and after treatment.

\section{Assessment of Quality of life}

Quality of life was assessed by means of the EORTC core questionnaire, the QLQ-C30 and the supplemental lung cancer specific module, the QLQ-LC13. The QLQ-C30 (version 1.0) contains 30 questions organized into five functional scales (physical, role, emotional, cognitive and social), three symptoms scales (fatigue, pain and nausea and vomiting), a global health/quality of life scale, a number of single items assessing additional symptoms (dyspnea, sleep disturbance, constipation and diarrhea), and perceived financial impact. This questionnaire has proven to be a valid and reliable tool when used among a wide range of cancer patient populations, including lung cancer patients ${ }^{5}$. It has been translated and validated for use with Dutch patients. The QLQ-LC13 contains 13 questions assessing disease-related and treatment-related symptoms frequently reported by patients with lung cancer ${ }^{22}$. Following procedures recommended by the EORTC, scores were linearly converted to a 0 to 100 scale. For the functional and global health status/quality of life scales, higher scores represent a better level of functioning. For the symptom scales, higher scores represent a greater degree of symptoms.

Quality of life and respiratory symptoms were assessed before the start of radiotherapy, in the fourth week during radiotherapy, and subsequently 2 weeks, 6 weeks, and 3, 6 and 12 months after the completion of radiotherapy.

Patients were asked to participate in the study during the first visit at the radiotherapy department. Following informed consent, the baseline questionnaire was distributed to the patients with a request to return it within 3 days by mail. During follow-up, the questionnaires were sent to the patients by mail. When the questionnaire was not returned within 4 days, the patient was telephoned and asked to complete and return it.

\section{Assessment of objective tumor response}

To assess objective tumor response, the product of the 2 largest diameters measured was calculated before and 2 to 6 weeks after radiotherapy using CT scan of the chest and/or 
chest radiography. In the majority of cases, a post-radiotherapy CT scan of the chest was available. In the cases in which only post-radiotherapy chest radiographs were available, only those with measurable disease were included in the analysis ${ }^{23}$. A response was defined as the complete disappearance of the tumor or as a reduction of $50 \%$ or more of the product of the two largest diameters. A reduction of less than $50 \%$ or progression was classified as non-response. A bronchoscopy to evaluate tumor response was not routinely performed.

\section{Assessment of radiation-induced pulmonary changes}

Radiation-induced pulmonary changes were assessed using chest radiographs made at 6 weeks and 3 months after radiotherapy. The changes were classified according to the SOMA scoring system ${ }^{24}$. According to this system, changes are classified into five categories: no changes; minimal changes; patchy changes; dense changes and severe changes.

\section{Statistics}

Changes in symptoms and QoL were evaluated in two different ways. First, a group-based analysis was performed analyzing changes of the mean scores over time with a repeated measurement ANOVA using a mixed effect modeling procedure, SAS Proc Mixed. In contrast to a "complete cases analysis", the mixed effect modeling retains in the analysis patients who dropout during follow-up. Trends over time for dropouts and complete cases are estimated under the assumption that all patients within the same group have the same change pattern over time. SAS Proc Mixed uses the method of restricted maximum likelihood to estimate the parameters of the model. F-tests are used for testing main effects of group and time, and an interaction effect of group $x$ time.

Second, the proportion of patients showing a response in symptoms and QoL was calculated. For the definition of response of symptoms, functioning and global QoL, a classification system derived from Stephens and co-workers was used ${ }^{25}$. This classification system has been described in detail in an earlier study ${ }^{20}$ and is outlined in appendix 1 (for symptoms) and 2 (for functioning and global QoL).

To assess the response rate for symptoms, each symptom scale was first divided into four categories. For the single-item scales (cough, haemoptysis, pain arm/shoulder, pain chest 
wall and appetite loss), four levels of scores were defined: $1=$ nil, $2=$ mild, $3=$ moderate and $4=$ severe. For the multi-item scales (dyspnea and fatigue), four levels of scores were defined as well, based on the converted score ranging from 0 to $100: 0=$ nil, 1 to $34=$ mild, 35 to $67=$ moderate and 68 to $100=$ severe. The chi-square statistic was used to analyze the association between objective tumor response and symptoms and QoL response.

Survival was determined from the first day of radiotherapy. Survival curves were estimated with the Kaplan-Meier method. Differences in survival outcome were tested for statistical significance with the log rank test. All results were regarded as statistically significant if the probability of chance was $5 \%$ or less.

\section{Results}

\section{Compliance}

Of the 167 patients who gave their informed consent, 164 returned the baseline questionnaire (98\%). Of the 155 patients still alive in the fourth week of radiotherapy, 147 (95\%) returned the questionnaire. After radiotherapy, the compliance was $91 \%$ at 2 weeks ( 133 out of 146 patients alive), $89 \%$ at 6 weeks ( 116 out of 130 patients alive) and $86 \%$ at 3 months (102 out of 119 patients alive), $84 \%$ at 6 months ( 71 out of 85 patients alive) and $87 \%$ at 12 months (39 out of 45 patients alive). The sample size of 147 is sufficient to detect a 10 point change in mean scores of the EORTC QLQ-C30 and the EORTC-LC13 scales with alpha set at 0.05 and a power ranging from 0.80 to 0.95 for all scales. A 10 point change in mean scale scores in the EORTC QLQ-C30 is considered to be a relatively small but clinically relevant change ${ }^{26}$.

No significant differences regarding pre-treatment characteristics (i.e. T- and $\mathrm{N}$ classification, WHO performance status, weight loss, age and sex) were observed between patients who completed all 12 months of QoL assessments and those who did not.

\section{Survival}

The median survival of this cohort of patients was 8.5 months (95\%-ci: 6.7-10.4 months). The 1-, 2- and 3-year survival was $39 \%, 16 \%$ and $8 \%$, respectively. The median survival of the patients with an objective tumor response was 13.1 months (95\%-ci: 11.0-15.2 
Table 1: Pre-treatment symptoms

\begin{tabular}{llcc}
\hline Symptom or functioning scale & & \% of patients & $\begin{array}{c}\text { Mean score in all } \\
\text { patients (SD) }\end{array}$ \\
\hline Symptoms & Cough & 91 & $48.9(25.4)$ \\
& Fatigue & 84 & $39.9(30.0)$ \\
& Dyspnea & 78 & $34.6(28.9)$ \\
& Insomnia & 56 & $33.7(34.8)$ \\
& Pain & 53 & $22.0(27.4)$ \\
& Appetite loss & 44 & $27.2(34.7)$ \\
& Chest pain & 38 & $17.1(24.9)$ \\
& Pain arm/shoulder & 36 & $21.3(32.0)$ \\
& Nausea and vomiting & 24 & $7.3(16.4)$ \\
& Heamoptysis & 22 & $9.2(19.0)$ \\
& Dysphagia & 18 & $8.7(20.5)$ \\
& Constipation & 18 & $8.7(21.2)$ \\
& Physical functioning & - & $61.8(26.6)$ \\
& Role functioning & - & $62.5(36.9)$ \\
& Emotional functioning & - & $64.9(28.5)$ \\
& Cognitive functioning & - & $83.8(22.6)$ \\
& Social functioning & - & $80.4(24.9)$ \\
& Global quality of life & - & $56.7(24.1)$ \\
\hline
\end{tabular}

months), and was significantly higher $(p<0.001)$ than the median survival of 7.9 months (95\%-co: 4.3-8.4 months) observed among those without an objective tumor response.

\section{Baseline symptoms and QoL}

The proportion of patients presenting with specific symptoms at study entry for the domains assessed with the QLQ-C30 and QLQ-LC13 are presented in Table 1. Fatigue $(84 \%)$, insomnia $(56 \%)$ and pain $(53 \%)$ were the most frequently reported general symptoms. Cough $(91 \%)$, dyspnea $(78 \%)$ and chest pain $(38 \%)$ were the most frequently reported respiratory symptoms.

Changes in mean symptom and QoL scores over time

The changes in the mean scores for the respiratory symptoms are presented in Figure 1a. The mean scores for cough and haemoptysis did not change significantly over time. Dyspnea worsened gradually with time. For chest pain and arm/shoulder pain, a gradual, 
Table 2: Response classification for general and respiratory symptoms $(n=164)$.

\begin{tabular}{lcrrrrrr}
\hline Symptom & $\begin{array}{c}\text { Inadequate } \\
\text { follow up } \\
\text { data }\end{array}$ & Improvement & Control & Prevention & $\begin{array}{l}\text { Dead without } \\
\text { palliation }\end{array}$ & Response rate & Worse \\
\hline Cough & 22 & $19 / 129(7 \%)$ & $25 / 62(40 \%)$ & $0 / 13(0 \%)$ & $27 / 142(19 \%)$ & $44 / 142(31 \%)$ & $30 / 142(21 \%)$ \\
Haemoptysis & 42 & $18 / 28(64 \%)$ & $1 / 21(5 \%)$ & $82 / 94(87 \%)$ & $7 / 122(6 \%)$ & $101 / 122(83 \%)$ & $2 / 122(2 \%)$ \\
Dyspneu & 28 & $17 / 109(16 \%)$ & $21 / 56(38 \%)$ & $12 / 27(44 \%)$ & $23 / 136(17 \%)$ & $50 / 136(37 \%)$ & $29 / 136(21 \%)$ \\
Pain arm/shoulder & 41 & $17 / 48(35 \%)$ & $6 / 21(29 \%)$ & $55 / 75(73 \%)$ & $10 / 123(8 \%)$ & $55 / 123(63 \%)$ & $16 / 123(13 \%)$ \\
Chest pain & 37 & $25 / 53(47 \%)$ & $18 / 38(47 \%)$ & $43 / 74(58 \%)$ & $12 / 127(9 \%)$ & $86 / 127(68 \%)$ & $20 / 127(16 \%)$ \\
Fatigue & 23 & $14 / 117(12 \%)$ & $19 / 56(34 \%)$ & $7 / 24(29 \%)$ & $27 / 141(19 \%)$ & $40 / 141(28 \%)$ & $36 / 141(26 \%)$ \\
Appetite loss & 36 & $27 / 59(44 \%)$ & $6 / 21(29 \%)$ & $44 / 69(64 \%)$ & $15 / 128(12 \%)$ & $76 / 128(59 \%)$ & $22 / 128(17 \%)$ \\
\hline
\end{tabular}

* Note: the number of patients with inadequate follow up data may differ between scales because of the definition of 'inadequate follow up' (see also table 2)

Table 3: Response classification for functioning and global quality of life ( $n=164)$.

\begin{tabular}{lccccccc}
\hline Symptom & $\begin{array}{c}\text { Inadequate } \\
\text { follow up } \\
\text { data }\end{array}$ & Improvement & Control & Prevention & $\begin{array}{c}\text { Dead without } \\
\text { palliation }\end{array}$ & Response rate & Worse \\
\hline PF & 28 & $11 / 85(13 \%)$ & $20 / 47(43 \%)$ & $17 / 51(33 \%)$ & $24 / 136(18 \%)$ & $48 / 136(35 \%)$ & $40 / 136(29 \%)$ \\
RF & 29 & $13 / 75(17 \%)$ & no patients & $34 / 120(28 \%)$ & $22 / 135(16 \%)$ & $47 / 135(35 \%)$ & $26 / 135(19 \%)$ \\
EF & 33 & $37 / 75(49 \%)$ & $2 / 22(9 \%)$ & $14 / 56(25 \%)$ & $18 / 131(14 \%)$ & $53 / 131(40 \%)$ & $41 / 131(31 \%)$ \\
CF & 34 & $13 / 29(45 \%)$ & $1 / 10(10 \%)$ & $58 / 101(57 \%)$ & $17 / 130(13 \%)$ & $72 / 130(55 \%)$ & $24 / 130(18 \%)$ \\
SF & 3 & $18 / 44(41 \%)$ & $2 / 20(10 \%)$ & $52 / 87(60 \%)$ & $18 / 131(14 \%)$ & $72 / 131(55 \%)$ & $28 / 131(21 \%)$ \\
Global QoL & 25 & $36 / 109(33 \%)$ & $4 / 30(13 \%)$ & $10 / 30(33 \%)$ & $25 / 139(18 \%)$ & $50 / 139(36 \%)$ & $34 / 139(24 \%)$ \\
\hline
\end{tabular}

* Note: the number of patients with inadequate follow up data may differ between scales because of the definition of 'inadequate follow up' (see also table 2).

significant increase was observed, particularly in the period between 6 and 12 months after the completion of radiotherapy.

The mean change scores for the general symptoms are shown in Figure $1 \mathrm{~b}$. Emesis and dysphagia were included in the analysis as well, because these symptoms were considered to be important, particularly during radiotherapy. For dysphagia, a significant increase during radiotherapy was observed, returning to baseline levels at 6 weeks. Fatigue and emesis increased significantly during radiotherapy followed by a decrease, although not to baseline levels. Appetite loss also increased significantly during radiotherapy, but decreased to a level lower than the baseline value at 6 weeks and 3 months. The mean scores for the functioning scales and global QoL scale are shown in 
Figure 1: Course of the mean scores for (1a) respiratory and (1b) general symptoms and the (1c) functioning and global quality scales.
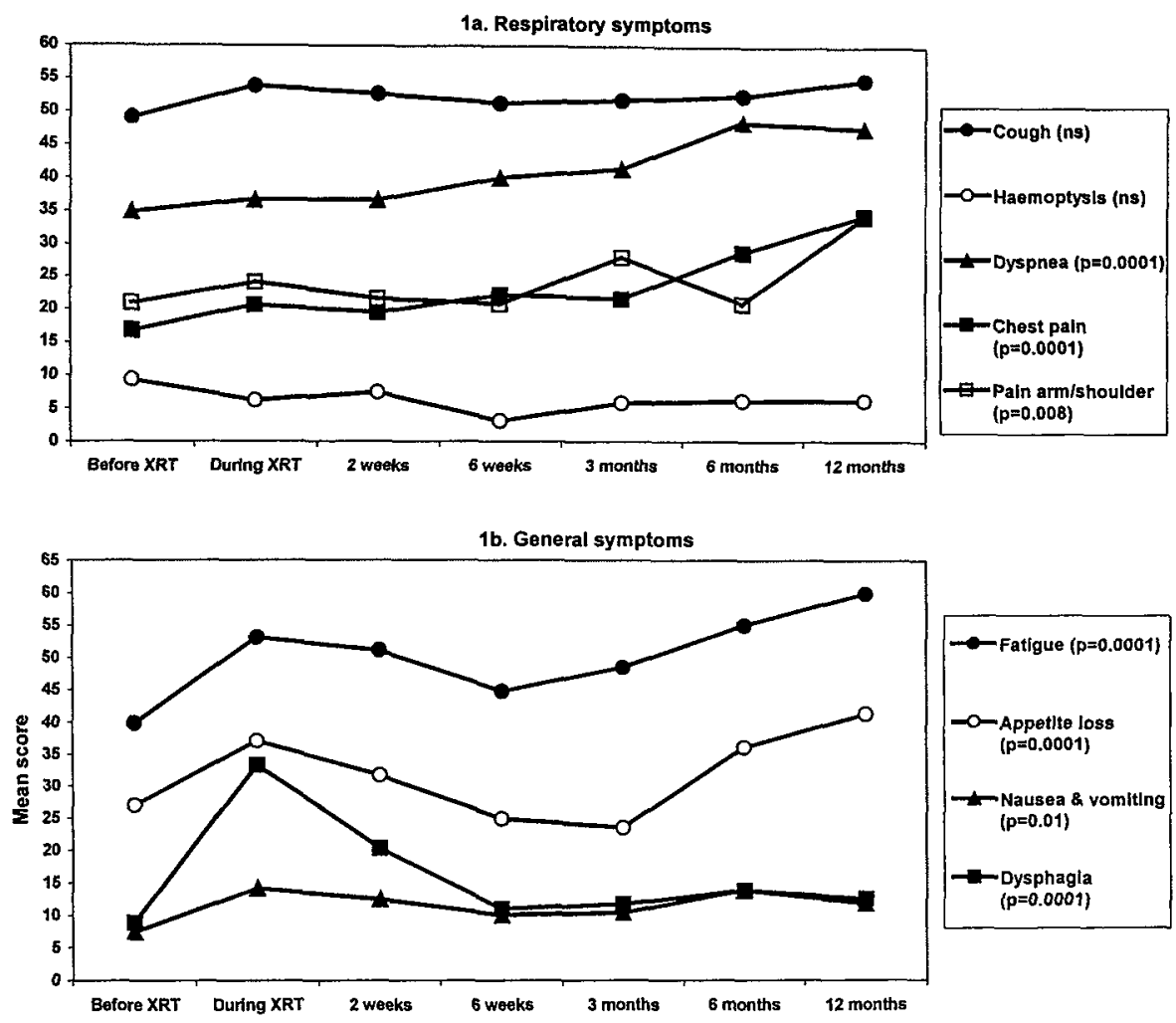

1c. Functioning and global QoL scales

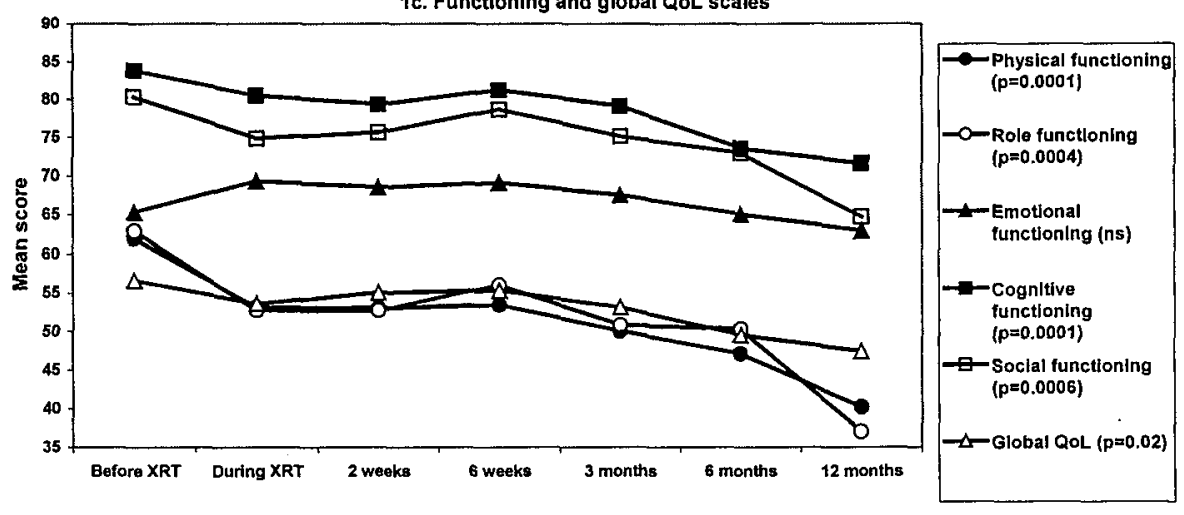

Note: The p-values refer to the comparison of the mean values observed before, during and after radiotherapy ( 2 weeks, 6 weeks and 3,6 and 12 months) calculated using the general linear model for repeated measurements.

${ }^{*} L=$ linear association

** $Q=$ quadratic association

$* * \mathrm{C}=$ cubic association 
Table 4: Quality of life response rates among objective tumor responders and non-responders.

\begin{tabular}{|c|c|c|c|}
\hline \multirow[t]{2}{*}{ Quality of life items } & \multicolumn{2}{|c|}{ Response rate QoL in: } & \multirow{2}{*}{$\begin{array}{c}\text { P-value } \\
\text { (2-sided chi- } \\
\text { Square) }\end{array}$} \\
\hline & $\begin{array}{l}\text { Objective tumor } \\
\text { responders }\end{array}$ & $\begin{array}{l}\text { Objective tumor } \\
\text { non-reponders }\end{array}$ & \\
\hline \multicolumn{4}{|l|}{ Respiratory symptoms } \\
\hline Cough & $33 / 79(42 \%)$ & $9 / 36(25 \%)$ & 0.08 \\
\hline Haemoptysis & $66 / 76(87 \%)$ & $21 / 27(78 \%)$ & 0.26 \\
\hline Dyspnea & $37 / 79(47 \%)$ & $9 / 32(28 \%)$ & 0.07 \\
\hline Pain arm/shoulder & $53 / 76(70 \%)$ & $12 / 28(43 \%)$ & 0.01 \\
\hline Pain chest wall & $50 / 76(66 \%)$ & $13 / 32(41 \%)$ & 0.02 \\
\hline \multicolumn{4}{|l|}{ General symptoms } \\
\hline Fatigue & $28 / 78(36 \%)$ & $7 / 37(19 \%)$ & 0.07 \\
\hline Appetite loss & $47 / 76(62 \%)$ & $20 / 30(67 \%)$ & 0.64 \\
\hline \multicolumn{4}{|l|}{ Functioning scales } \\
\hline Physical functioning & $36 / 76(47 \%)$ & $8 / 36(22 \%)$ & 0.01 \\
\hline Role functioning & $33 / 75(44 \%)$ & $9 / 35(26 \%)$ & 0.07 \\
\hline Emotional functioning & $32 / 75(43 \%)$ & $15 / 30(50 \%)$ & 0.50 \\
\hline Cognitive functioning & $47 / 76(62 \%)$ & $15 / 30(50 \%)$ & 0.27 \\
\hline Social functioning & $46 / 77(60 \%)$ & $17 / 30(57 \%)$ & 0.77 \\
\hline Global Quality of life & $33 / 78(42 \%)$ & $11 / 35(31 \%)$ & 0.27 \\
\hline
\end{tabular}

Figure 1c. A significant, gradual decrease was observed for physical and role functioning and global QoL, particularly during radiotherapy and in the period between 6 and 12 months after the completion of radiotherapy. Social functioning worsened during radiotherapy, improved at 6 weeks and worsened again after 3 months to a level which was significantly lower than baseline. No statistically significant changes were observed for emotional functioning. 


\section{Symptom and quality of life response}

A. wide variation in the response rates for respiratory symptoms was observed (Table 2). The highest response rate was observed for haemoptysis $(83 \%)$. The response rates for chest pain and pain in the arm/shoulder were $68 \%$ and $61 \%$, respectively. Much lower response rates were observed for cough and dyspnea, i.e. $31 \%$ and $37 \%$, respectively. In a proportion of patients, respiratory symptoms worsened. The rate of deterioration was highest for cough (21\%) and dyspnea (21\%). For fatigue, the response rate was $28 \%$ and for appetite loss, $59 \%$ (Table 2).

Physical functioning, role functioning and global $\mathrm{QoL}$ improved in approximately one-third of the patients (Table 3 ). The highest response rates were observed for social and cognitive functioning (55\%). The response rate for emotional functioning was $40 \%$. Worsening of different functioning scales and global QoL was observed in $18 \%$ (for cognitive functioning) to $31 \%$ (for emotional functioning) of the cases.

\section{Changes in QoL in tumor responders and non-responders}

Objective tumor response could be determined in 130 of the 164 cases $(79 \%)$. In 90 patients $(69 \%)$, an objective tumor response was observed, while 40 patients $(31 \%)$ were classified as non-responders.

The possible association between objective tumor response and changes in symptoms and QoL was examined in two different ways. First, differences in QoL mean scores over time between objective tumor responders and non-responders were analyzed. Second, the differences in the response rates for all symptom and QoL scales were compared between the objective tumor responders and tumor non-responders.

with repeated measure ANOVA using the mixed model procedure, no significant differences in the mean scores were observed between the objective responders and nonresponders, except for chest wall pain ( $p=0.03$ ) (Figure 2 ).

However, a significant association was observed between objective tumor response and symptom response for chest wall pain and arm/shoulder pain (Table 4). In the group of patients classified as having an objective tumor response, the response rate for pain in the arm/shoulder was $70 \%$, compared to $43 \%$ in those without an objective tumor response ( $p$ $=0.01$ ). For pain in the chest wall, comparable results were observed $(p=0.02)$. A borderline significant $(p<0.10)$ association was found between objective tumor response 
Figure 2: Mean scores for chest wall pain stratified by objective tumor response.

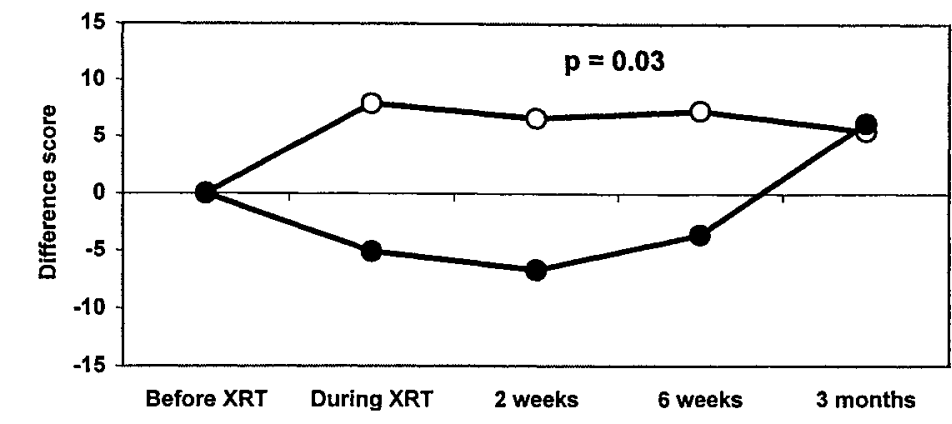

White marker $=$ no objective tumor response

Black marker $=$ objective tumor response

and response for self-reported cough, dyspnea and fatigue. For the other symptom rates, no significant associations with objective tumor response were observed.

For physical functioning, the response rate in the objective responders was $47 \%$ compared to $22 \%$ in those without an objective tumor response $(p=0.01)$. No significant association was found between the other functioning scales and global QoL scale and objective tumor response (Table 6).

Relation between symptoms, quality of life and radiation-induced pulmonary changes Information about radiation-induced changes in the first three months after the completion of radiotherapy was available for 107 patients. In 69 patients $(65 \%)$ no changes or only minimal changes were observed, in 24 patients $(22 \%)$ patchy changes were seen on the chest radiographs, while in 14 patients $(13 \%)$ dense or severe changes were detected. The mean scores for dyspnea increased more in patients who developed radiationinduced pulmonary changes compared to those with no or minimal changes. However, this difference did not reach statistical significance (Figure 3). No significant association was found with the other symptoms or with the functioning and global QoL scales. In the subject-based analysis, dyspnea and fatigue were found to be associated significantly with radiation-induced pulmonary changes. In case of no or minimal changes, 31 out of 61 
Figure 3: Mean scores for dyspnea stratified by radiation-induced changes with chest radiograph.

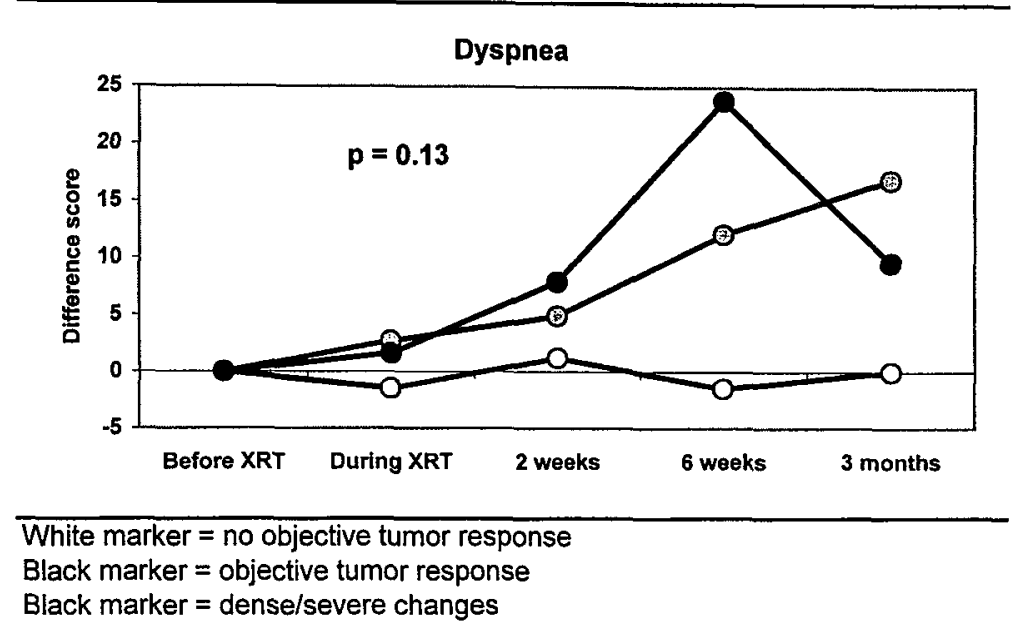

patients $(51 \%)$ had a response for dyspnea, in case of patchy changes, 9 out of 21 patients $(43 \%)$, and in case of dense/severe changes, 3 out of 13 patients $(23 \%)(p=0.04)$. Worsening of dyspnea was observed in $18 \%, 29 \%$ and $46 \%$ of these patients, respectively. Twenty-five of 62 patients $(40 \%)$ with no or minimal radiographic changes had a response for fatigue. The corresponding figures were 8 of 22 patients $(36 \%)$ for those with patchy changes and one of 13 patients $(8 \%)$ for those with dense or severe changes. Worsening of dyspnea was observed in $23 \%, 41 \%$ and $54 \%$ of these patients, respectively $(p=0.01)$. No significant association was found with the other symptoms or with the functioning and global $Q \mathrm{OL}$ scales.

\section{Discussion}

The prognosis in patients with inoperable NSCLC remains poor, despite radical radiotherapy. In the present study, the median survival was approximately 8.5 months, with just $8 \%$ of the patients still alive after 3 years. These figures are comparable with those reported by others ${ }^{1-4}$. Taking into account this relatively short survival, both 
oncologists and their patients need information about palliation of symptoms and changes in QoL resulting from radiotherapy.

In the present study, significantly higher response rates for arm/shoulder pain, chest pain and physical functioning were found among the objective tumor responders compared to those without an objective tumor response. Comparison of the mean scores for the symptom and QoL scales did not reveal any significant differences between the objective tumor responders and non-responders, except for chest pain. These findings illustrate that sole reliance on analyses comparing the mean scores between groups can mask subjectspecific differences as revealed when comparing response rates. The current findings indicate that, as a result of tumor reduction, some respiratory symptoms are palliated. In one of our previous studies among patients with NSCLC treated with palliative radiotherapy, an association between tumor response and palliation of symptoms was also found ${ }^{20}$. Contrary to the results concerning symptom palliation, no such association was found between the functioning scales, global QoL and tumor response, except for physical functioning. In small cell lung cancer (SCLC), an association between tumor response and quality of life was reported by Wolf and co-workers in a study of 321 patients treated with chemotherapy ${ }^{27}$. However, for NSCLC, such an association has never been described. These findings also demonstrate that objective tumor response can not serve as a surrogate for improvement of symptoms and/or QoL. Palliation of symptoms or improvement of QoL was achieved in a considerable proportion of patients without tumor response, Conversely, in a relatively large proportion of patients in which tumor response was observed, no palliation of symptoms was achieved. This might be explained by the fact that the majority of patients with NSCLC are current smokers or exsmokers. In this study, the majority of patients had a background of pulmonary symptoms due to diffuse airway disease or other comorbid diseases such as congestive heart failure, which pre-date and persist alongside the local effects of the tumor. Moreover, the side effects of radiotherapy may counteract any decrease of respiratory symptoms achieved by tumor reduction.

The putative association between radiation-induced radiological changes and respiratory complaints and in particular with dyspnea, was clearly demonstrated in the present study. Significantly higher response rates and lower progression rates for dyspnea were observed in those without versus those with radiation-induced pulmonary changes. 
Radiation-induced pulmonary changes assessed on chest radiographs at 6 weeks and 3 months after radiotherapy were preceded by an increase of dyspnea during radiotherapy, while at that time no abnormalities could be detected on the chest radiograph. An otherwise unexplained increase of dyspnea during radiotherapy might therefore be predictive of the development of radiation pneumonitis and/or fibrosis. Taking into account the high incidence of patients with smoking-related comorbid diseases, the question arises as to whether smoking cessation might minimize radiation-induced side effects even during palliation for advanced stage disease. One of the limitations of the present study is that no reliable information regarding the smoking history or ongoing tobacco use is available. With the increasing discussion of the importance of smoking cessation during treatment, smoking habits before, during and after treatment should be systematically assessed in future studies investigating QoL among lung cancer patients.

The current results indicate that good palliation is achieved for haemoptysis and, to a lesser extent, for pain in the chest and in the arm/shoulder.

One of the potential pitfalls of the QoL response classification system used in the current analysis is that defining 'prevention' as response assumes that symptoms that are not present at the onset of treatment would have occurred if the radiotherapy had not been administered. This will not be the case in all patients and for all symptoms. However, assuming the opposite (i.e. none of the patients without symptoms at the onset of treatment would have developed any symptom if the radiotherapy had not been administered), is also very unlikely. To define these latter patients as having had 'no response' would skew the results in the opposite direction Thus in reporting of response rates as proposed by Stephens requires detailed information on prevention, control and improvement. Without doing so, one runs the risk in overestimating the beneficial effect of radiotherapy, particularly for those symptoms with a low incidence at the onset of treatment.

The most frequently reported respiratory symptoms, cough and dyspnea, were not well palliated with radical radiotherapy. The reduction in respiratory symptoms in the current study was similar to that reported in one of our previous studies of palliative radiotherapy (30 Gy) ${ }^{20}$. In two MRC studies ${ }^{15-16}$, no significant differences in palliation of respiratory symptoms were observed between conventional palliative fractionation (10 $\times 3 \mathrm{~Gy}$ ) and two hypofractionated schedules ( $2 \times 8.5$ Gy with a one week interval and $1 \times 10 \mathrm{~Gy})$. The 
response rates for the respiratory symptoms in these two studies were comparable to those observed in the current study, suggesting that palliation of respiratory symptoms and QoL effects do not depend on the total dose administered.

In the present study, the mean values for most respiratory symptoms, including dyspnea, chest pain and pain in the arm/shoulder increased over time. Fatigue also increased with time, particularly during radiotherapy. A gradual increase of respiratory symptoms in patients treated for NSCLC was also observed in two other studies. Rees and co-workers reported the results of a prospective, randomized study comparing two palliative schedules 17. The majority of these patients had NSCLC and all were treated for palliation only. For all respiratory symptoms, the cumulative proportion of patients reporting improvement decreased with time. Muers and co-workers found the same gradual increase in symptoms among patients with NSCLC treated palliatively with different modalities $^{14}$.

For the functioning scales, a clear deterioration was observed during radiotherapy for physical and role functioning and, to a lesser extent, for global QoL. The worsening of functioning during radiotherapy is probably related to the treatment itself and also reflects an increase in general symptoms observed during radiotherapy. These results suggest that, although palliation and improvement of QoL can be achieved in a certain proportion of patients (as expressed by the response rates), there is a gradual worsening of most symptoms and functioning levels over time.

The only exception to this pattern was found for emotional functioning, which improved slightly during treatment and then gradually returned to the baseline level during follow up. A possible explanation for the improvement in emotional functioning during treatment is that being on active treatment in and of itself has a salutary effect on mood. Conversely, the end of active treatment may be accompanied by a loss of hope and thus a lower level of emotional functioning.

We would note that the results reported here are based on a sample of patients of NSCLC who had received radiotherapy. Ideally, one would want to compare these results with those obtained from a comparative group of patients who had not received radiotherapy (e.g. in a randomized controlled study). However, given the relatively good prognosis of this patient population, and the fact that radiotherapy or chemoradiation is universally 
accepted as the standard treatment, such a comparative study would be neither ethical nor practical.

In conclusion, radical radiotherapy offers excellent palliation of haemoptysis, good palliation of pain and poor palliation of cough and dyspnea. The response rates for different QoL domains varied between $34 \%$ for physical functioning and $51 \%$ for social functioning. Tumor reduction plays a role in palliation of some respiratory symptoms, with the exception of haemoptysis. Despite this significant association, objective tumor response can not serve as a surrogate for palliation or improvement of $\mathrm{QoL}$. 


\section{References}

1. Perez CA, Stanley $K$, Grundy $G$, et al. Impact of irradiation technique and tumor extent in tumor control of patients with unresectable non-oat cell carcinoma of the lung. Cancer 1982; 50: 1091-1099.

2. Bulzebruck, $H$, Bopp $R$, Drings $P$, et al. New aspects in the staging of lung cancer. Prospective validation of the International Union Against Cancer TNM Classification. Cancer 1992; 70: 1102-1110.

3. Cox JD, Azarnia N, Byhardt RW, et al. N2 (clinical) non-small cell carcinoma of the lung: Prospective trials of radiation therapy with total doses of 60 Gy by the Radiation Therapy Oncology Group. Int J Radiat Oncol Biol Phys 1991; 20: 712.

4. Curran WJ, and Stafford PM. Lack of apparent differences in outcome between clinically staged IIIA and IIIB non-small cell lung carcinoma treated with radiotherapy. $J$ Clin Oncol 1990; 8: 409-415.

5. Aaronson NK, Ahmedzai S, Bergman B, et al. The European Organization for Research and Treatment of Cancer QLQ-C30: a quality of life instrument for use in international clinical trials in oncology. J Natl Cancer Inst 1993; 85: 365376.

6. Schag CA, Ganz PA, and Heinrich RL. Cancer, Rehabilitation Evaluation System-short form (CARES-SF). A cancer specific rehabilitation and quality of life instrument. Cancer 1991; 68: 1406-1413.

7. Te Velde A, Sprangers MA, and Aaronson NK. Feasibility, psychometric performance, and stability across modes of administration of the CARES-SF. Ann Oncol 1996; 7: 381-390.

8. Fayers $P M$, Bleehen NM, Girling DJ, and Stephens RJ: Assessment of quality of life in small cell lung cancer using a daily diary card developed by the Medical Research Council Working Party. Br J Cancer 1991; 64: 299-306.

9. Schipper $\mathrm{H}$, Clinch $J$, McMurray $A$, et al. Measuring quality of life of cancer patients: The Functional Living IndexCancer: Development and validation. J Clin Oncol 1984; 2: 472-483.

10. Spitzer WO, Dobson AJ, Hall J, et al. Measuring the quality of life of cancer patients. Chron Dis $1981 ; 34: 585-597$.

11. Cella DF, Tulsky DS, Gray G, et al. The functional Assessment of Cancer Therapy Scale: Development and validation of the general measure. J Clin Oncol 1993; 11: 570-579.

12. Cella DF, Bonomi AE, Lloyd SR, et al. Reliability and validity of the Functional Assessment of Cancer Therapy-Lung (FACT-L) quality of life instrument. Lung Cancer 1995; 12: 199-220.

13. Hollen PJ, Gralla RJ, Kris MG, et al. Quality of life assessment in individuals with lung cancer: Testing the Lung Cancer Symptom Scale (LCSS). Eur J Cancer 1993; 29 A (suppl 1): S51-S58.

14. Muers MF, and Round CE. Palliation of symptoms in non-small cell lung cancer: a study by the Yorkshire Regional Cancer Organisation thoracic group. Thorax 1993; 48: 339-343.

15. Medical Research Council Lung Cancer Working Party. Inoperable non-small cell lung cancer (NSCLC): a Medical Research Counsel randomized trial of palliative radiotherapy with two or ten fractions. $\mathrm{Br} J$ Cancer 1991; 63: 265-270.

16. Medical Research Council Lung Cancer Working Party. A Medical Research Counsel (MRC) randomized trials of palliative radiotherapy with two fractions or a single fraction in patients with inoperable non-small cell lung cancer (NSCLC) and poor performance status. $\mathrm{Br} J$ Cancer 1992; 65: 934-941.

17. Rees GJG, Devrell CE, Barley VL, and Newman HFV. Palliative radiotherapy for lung cancer: two versus five fractions. Clin Oncol 1997; 9: 90-95.

18. Helsing $M$, Bergman $B$, Thaning $L$. et al. Quality of life and survival in patients with advanced non-small cell lung cancer receiving supportive care plus chemotherapy with carboplatin and etoposide or supportive care only. A multicentre randomised phase III trial. Eur J Cancer 1998; 34(7): 1036-1044. 
19. Lutz ST, Huang DT, Ferguson CL, et al. A retrospective quality of life analysis using the Lung Cancer Symptom Scale in patients treated with palliative radiotherapy for advanced nonsmall cell lung cancer. Int $\mathrm{J}$ Radiot Oncol Biol Phys 1997; 37: 117-122.

20. Langendijk JA, ten Velde GPM, Aaronson NK, et al. Quality of life after palliative radiotherapy in non-small cell lung cancer: a prospective study. Int J Radiat Oncol Biol Phys 2000; 47: 149-155.

21. In Hermanek $P$ and Sobin LH (Ed) In: TNM Atlas: Classification of malignant tumours; Springer-Verlag, 1992: pp 7584.

22. Bergman B, Aaronson NK, Ahmedzai S, et al. The EORTC QLQ-LC13: A modular supplement to the EORTC Core Quality of Life Questionnaire (QLQ-C30) for use in clinical trials in oncology. Eur J Cancer 1994; 30A: 635-642.

23. Langendijk JA, Lamers RJS, ten Velde GPM, et al. is the chest radiograph a reliable tool in the assessment of tumor response after radiotherapy in nonsmall cell lung carcinoma. Int J Radiat Oncol Biol Phys 41: 1037-1045, 1998.

24. Pavy JJ, Denekamp J, Letschert J, et al. EORTC Late Effects Working Group. Late effects toxicity scoring: the SOMA scale. Radiother Oncol 1995; 35:11-15.

25. Stephens RJ, Hopwood P, and Girling DJ. Defining and analysing symptom palliation in cancer clinical trials: a deceptively difficult exercise. Br J Cancer 1999; 79: 538-544.

26. Osoba $D$, Rodrigues $G$, Myles $\mathrm{J}$, et al. Interpreting the significance of changes in quality of life scores. $\mathrm{J}$ Clin Oncol 1998; 16: 139-44.

27. Wolf $M$, Pritsch $M$, Drings $P$. et al. Cyclic-alternating versus response-oriented chemotherapy in small-cell lung cancer: a German multicentre randomized trial of 321 patients. J Clin Oncol 1991; 9: 614-624. 


\section{Appendix 1}

\section{Response criteria for general and respiratory symptoms}

'Response' is defined as:

- Baseline score 'moderate or severe', with improvement to 'mild' or 'nil' $t=$ 'improvement'.

- Baseline score 'mild', with improvement to 'nil' $f=$ 'improvement'.

- Baseline score 'mild', with 'mild' $t=$ 'control'.

- Baseline score 'nil', with 'nil' $\dagger=$ 'prevention'.

'No response' is defined as:

- Baseline score 'moderate or severe', without improvement to 'mild or nil' $t=$ 'no change'.

- Baseline score 'moderate or severe' and dead before first assessment after the end of radiotherapy = 'dead without palliation'.

- Baseline score 'moderate or severe' without improvement to 'mild or nil' on the first assessment and dead before the second assessment after the end of radiotherapy = 'dead without palliation'.

- Baseline score 'mild', without improvement to 'nil' and without deterioration to 'moderate or severe' $t=$ 'no change'.

- Baseline score 'mild' and dead before first assessment after the end of radiotherapy = 'dead without palliation'.

- Baseline score 'mild', without improvement to 'nil' on the first assessment and dead before first assessment after the end of radiotherapy $=$ 'dead without palliation'.

- Baseline score 'nil', without 'nil' and without deterioration to 'mild, moderate or severe' $\dagger=$ 'no change'.

- Baseline score 'nil', with deterioration to 'mild, moderate or severe' on the first assessment and dead before the second assessment after the end of radiotherapy = 'dead without palliation'.

'Progression' is defined as:

- Baseline score 'mild', with deterioration to 'moderate or severe' $\dagger$.

- Baseline score 'nil', with 'mild, moderate or severe' $\dagger$.

'Not evaluable' is defined as:

- Baseline score 'moderate or severe', with improvement to 'nil or mild' on the first assessments and dead before the second assessment after the end of radiotherapy.

- Baseline score 'mild', with improvement to 'nil' on the first assessment and dead before the second assessment after the end of radiotherapy.

- Baseline score 'nil' and dead before the first assessment after the end of radiotherapy.

- Baseline score 'nil', with 'nil' at the first assessment and dead before the second assessment after the end of radiotherapy.

\section{$\dagger=$ on at least two consecutive assessments in the first three months after the end of radlotherapy}




\section{Appendix 2}

\section{Response criteria for functioning scales and global quality of life}

\section{'Response' is defined as:}

- Baseline score ' $0-59$ ', with improvement of at least 5 points $\dagger$ to a minimal value of $40=$ 'improvement'.

- Baseline score ' $60-79$ ', with improvement of at least 5 points $\dagger=$ 'improvement'

- Baseline score ' $60-79$ ', with no change (i.e. $<5$ points) $\dagger=$ 'control'

- Baseline score ' $\underline{80-100}$ ', with improvement or no change (i.e. $<5$ points) $\dagger=$ 'prevention'

\section{'No response' is defined as:}

- Baseline score ' $0-59$ ', with no change (l.e. $<5$ points) $\dagger=$ 'no change'

- Baseline score ' $0-59$ ' and dead before first assessment after the end of radiotherapy $=$ 'dead without palliation'.

- Baseline score ' $0-59$ ' without improvement of at least 5 points to a minimal value of 40 on the first assessment and dead before the second assessment after the end of radiotherapy = 'dead without palliation'.

- Baseline score ' $60-79$ ' and dead before first assessment after the end of radiotherapy = 'dead without palliation'.

- Baseline score ' $60-79$ ' without improvement of at least 5 points on the first assessment and dead before the second assessment after the end of radiotherapy $=$ 'dead without palliation'.

- Baseline score ' $80-100$ ', and dead before first assessment after the end of radiotherapy = 'dead without palliation'.

- Baseline score ' $80-100$ ' without improvement of at least 5 points on the first assessment and dead before the second assessment after the end of radiotherapy = 'dead without palliation'.

\section{'Progression' is defined as:}

- Baseline score ' $0-59$ ', with a decrease of at least 5 points $\dagger$

- Baseline score ' $60-79$ ', with a decrease of at least 5 points $\dagger$

- Baseline score ' $80-100$ ', with a decrease of at least 5 points $\dagger$

\section{'Not evaluable' is defined as:}

- Baseline score ' $\underline{0-59}$, with improvement of at least 5 points to a minimal value of 40 on the first assessments and dead before the second assessment after the end of radiotherapy.

- Baseline score ' $60-79$ ', with improvement of at least 5 points on the first assessment and dead before the second assessment after the end of radiotherapy.

- Baseline score ' $80-100$ ', with improvement of at least 5 points on the first assessment and dead before the second assessment after the end of radiotherapy.

$t=$ on at least two consecutive assessments in the first three months after the end of radiotherapy 
Chapter 8

\title{
Quality of life after curative radiotherapy in stage I non- small cell lung cancer
}

\author{
Hans Langendijk \\ Neil Aaronson \\ Jos de Jong \\ Guul ten Velde \\ Martin Muller \\ Ben Slotman \\ Miel Wouters
}

Submitted 


\section{Abstract}

Purpose: The aim of this study was to investigate changes in quality of life (QOL) besides the traditional endpoints in medically inoperable stage I non-small cell lung cancer (NSCLC) treated with curative radiotherapy.

Patients and methods: Included were 35 patients with cytological or histologic confirmation of stage I NSCLC. QoL was assessed before, during and after radiotherapy, using the EORTC QLQ-C30 and QLQ-LC13. Twenty patients were only treated at the primary site while in 15 patients, the regional lymph nodes were included in the target volume as well. Results: The median survival was 19.5 months. None of the locally treated patients developed regional recurrence. A significant gradual increase with time was observed for dyspnea, chest pain and fatigue. A significant gradual deterioration was also observed for global QoL. No significant changes were observed for either the other symptoms nor the functioning scales. Significantly higher levels of dysphagia were observed in those in which the regional lymph nodes were treated as well compared to the locally treated patients, which persisted up to 12 months. Radiation-induced pulmonary changes assessed with chest radiograph were more pronounced in the group treated with locoregional radiotherapy.

Conclusions: After curative radiotherapy for stage I medically inoperable NSCLC, a gradual increase of dyspnea and chest pain is observed together with a significant deterioration of global QoL, possibly due to pre-existing, slowly progressive chronic obstructive pulmonary disease and radiation-induced pulmonary changes. Taking into account the low incidence of regional recurrences after local irradiation, the higher incidence and severity of radiation-induced changes and the higher levels of dysphagia persisting up to 12 months, local irradiation should be considered in small peripherally located tumours. 


\section{$\square$ Introduction}

Surgery is the treatment of choice in stage $I^{1}$ non-small cell lung carcinoma (NSCLC). Radiotherapy is considered a good alternative to surgery for medically inoperable patients with technically resectable tumours. The 5-year survival in stage I NSCLC achieved with radiotherapy varies from $6 \%$ to $27 \%{ }^{2-13}$. Besides the traditional endpoints such as response, loco-regional control and survival, quality of life (QoL) has been recognised as an important endpoint in clinical studies. In most studies, reporting on QoL in NSCLC, only patients with locally advanced or metastatic disease are involved who have been treated with palliative intent. To our knowledge, there are no clinical studies available using QoL as outcome determinant in patients with early stage NSCLC treated with curative radiotherapy.

The aim of this prospective study was to evaluate changes in QOL in patients with stage I NSCLC treated with curative radiotherapy Furthermore, the traditional endpoints, including response, loco-regional tumour control, distant metastases and survival were evaluated. Regarding QoL, one specific hypothesis was investigated. The population of patients treated for stage I NSCLC with radiotherapy generally have a high background incidence of pulmonary symptoms due to co-morbidity, which pre-dates and persists alongside the local effects of the tumour. Taking into account the relatively small size of most tumours, which are less likely to cause severe respiratory and/or general symptoms, the first hypothesis was that the mean scores for most symptoms would not change significantly when time passed, in particular in the first 12 months after radiotherapy. Additionally, we expected no significant changes regarding functioning and global QoL in the same period.

\section{Material and methods}

\section{Patient eligibility and characteristics}

In this prospective study, patients with NSCLC treated between June 1994 and December 1996 with curative radiotherapy were included. The inclusion criteria for curative radiotherapy were medically inoperable stage I (UICC 1997) disease ${ }^{1}$ with a maximum 
Table 1: Pre-treatment characteristics

\begin{tabular}{|c|c|c|c|}
\hline Variables & & $n$ & $\%$ \\
\hline \multirow[t]{2}{*}{ Sex } & Male & 31 & 89 \\
\hline & Female & 4 & 11 \\
\hline \multirow[t]{2}{*}{ T-classification } & T1 & 17 & 49 \\
\hline & $\mathrm{T} 2$ & 18 & 51 \\
\hline \multirow[t]{3}{*}{ Tumour type } & Squamous cell & 23 & 66 \\
\hline & Adeno & 2 & 6 \\
\hline & Undifferentiated & 10 & 29 \\
\hline \multirow[t]{3}{*}{ Weight loss } & none & 30 & 86 \\
\hline & $1-10 \%$ & 2 & 6 \\
\hline & $>10 \%$ & 3 & 9 \\
\hline \multirow[t]{3}{*}{ Performance status } & WHOO & 20 & 57 \\
\hline & WHO 1 & 13 & 37 \\
\hline & WHO 2 & 2 & 6 \\
\hline \multirow[t]{2}{*}{ Site } & Peripherally located & 26 & 74 \\
\hline & Centrally located & 9 & 26 \\
\hline
\end{tabular}

diameter of four centimetre and a WHO performance status $\leq 2$. In the aforementioned period, 35 patients were included in the study. Histologic or cytological confirmation was available in all patients. Patients' age varied from 47 to 82 years (median 72 years). The pre-treatment characteristics of these 35 patients are presented in Table 1. Thirty patients $(86 \%)$ were considered inoperable because of impaired lung function, 8 patients $(23 \%)$ because of other co-morbidity and 2 patients $(6 \%)$ because of age. In some patients, more than one reason was present. One patient $(3 \%)$ refused surgery. The most frequently reported co-morbid diseases were chronic obstructive pulmonary disease in 28 patients $(80 \%)$, a myocardial infarction in the past with ischemic heart disease in 12 patients (34\%) and/or congestive heart failure in 9 patients $(26 \%)$. 


\section{Radiotherapy protocol}

The clinical target volume (CTV) of the initial field included the gross tumour volume (GTV) with a 1.5 centimetre margin in all directions, the ipsilateral hilar lymph nodes and the mediastinal lymph nodes on both sides. The planning target volume (PTV) of the initial field encompassed the CTV with a 0.5 centimetre margin. In some cases, the cranialcaudal margin was increased because of movements due to respiration. The PTV of the initial field was treated with opposing anterior-posterior/posterior-anterior fields, using fraction doses of $2 \mathrm{~Gy}$ ( 5 times a week) to a total dose of $46 \mathrm{~Gy}$. In the majority of patients $(57 \%)$, the elective node areas were not included in the PTV of the initial field. In general, these patients had small peripherally located tumours and a very poor lung function due to co-morbid respiratory diseases. In these cases, a three- or four-field technique was used.

The CTV of the boost included the GTV with a 0.5 centimetre margin. The margin for the PTV was determined similarly as described for the initial field. The PTV of the boost was treated with a three- or four-field technique, using fraction doses of $2 \mathrm{~Gy}$ ( 5 times a week) to a total dose of $70 \mathrm{~Gy}$.

In all patients, CT-assisted planning was used. Individually customised lead blocking was used to prevent radiation to the normal tissue as much as possible. Corrections were made for lung tissue density (0.3). The dose was specified in the isocenter of the fields and the PTV received at least $90 \%$ of the prescribed dose.

\section{Assessment of Quality of life}

Quality of life was assessed by means of the EORTC core questionnaire QLQ-C30 and the supplemental lung cancer specific module, the QLQ-LC13. The QLQ-C30 (version 1.0) contains 30 questions addressing various aspects of $\mathrm{QoL}$. These questionnaire has been proved to be a valid and reliable tool used among a wide range of cancer patient populations, including lung cancer patients ${ }^{14,15}$. All scores were linearly converted to a 0 to 100 scale. For the functional and global health status/quality of life scales, higher scores represent a better level of functioning. For the symptom scales, higher scores represent a greater degree of symptoms. 
Quality of life and respiratory symptoms were assessed before the start of radiotherapy, in the fourth week during radiotherapy and subsequently 2 weeks, 6 weeks, 3, 6 and 12 months after the end of radiotherapy.

After informed consent, the baseline questionnaire was distributed to the patients. They were asked to return the questionnaire within 3 days by mail. During follow up, the questionnaires were sent to the patients by mail. When the questionnaire was not returned within 4 days, the patient was telephoned and asked to complete and return the questionnaire.

\section{Assessment of radiation-induced pulmonary changes}

Radiation-induced pulmonary changes were assessed using chest radiograph. The changes were classified according to the objective scale of the SOMA scoring system ${ }^{16}$. According to this system, changes are classified into five categories: no changes; minimal changes; patchy changes; dense changes and severe changes.

\section{Statistics}

Changes in symptoms and QoL were evaluated using the general linear model (GLM) for repeated measure analysis of variance (ANOVA), testing changes of the mean values of a group over time. In this analysis, only the complete cases were taken into account. Changes occurred in three different patterns: linear (i.e. a gradual increase or decrease over time); quadratic (i.e. an initial decrease followed by an increase to the baseline level, or an initial increase followed by a decrease to the baseline level); or cubic (an initial decrease followed by an increase to a value different from the baseline level, or an initial increase followed by a decrease to a value different from baseline)

The proportion of patients showing a response in symptoms and QoL was calculated as well. In summary, to assess the response rate for symptoms, each symptom scale was divided into four categories. For the single-item scales (cough, haemoptysis, pain arm/shoulder, pain chest wall and appetite loss), four levels of scores were defined: 1 = nil, $2=$ mild, $3=$ moderate and $4=$ severe. For the multi-item scales (dyspnea and fatigue), four levels of scores were defined as well, based on the converted score ranging from 0 to 100 : $0=$ nil, 1 to $34=$ mild, 35 to $67=$ moderate and 68 to $100=$ severe. 
In most studies, palliation is regarded as adequate in case of improvement of a symptom at a certain time point to a lower level of severity. We feel that a more appropriate index in the setting of NSCLC may be to regard palliation as adequate if severe or moderate symptoms are at least reduced to mild or absent and mild or absent symptoms remain and do not worsen for a certain period of time. The definition of response of symptoms and QoL has been described in detail in one of our previous studies ${ }^{17}$ and is summarised in appendix 1 (symptoms) and 2 (functioning and global QoL).

Survival was determined from the first day of radiotherapy. Survival was estimated with the Kaplan-Meier method and differences in survival outcome were tested on statistical significance with the log rank test. Loco-regional control was defined as the absence of progression of the primary tumour or the appearance of regional lymph node metastases and was calculated from the first day of radiotherapy.

All results were regarded as statistically significant if the probability of chance was $5 \%$ or less.

\section{Results}

\section{Objective tumour response}

Objective tumour response was assessed 2 to 6 weeks after the end of radiotherapy using CT scan of the chest or chest radiograph. A complete response (CR) was observed in 12 out of 30 measurable cases $(40 \%){ }^{18}$, a partial response (PR) in 12 cases $(40 \%)$ and stable disease (SD) in six cases $(20 \%)$. In the group with T1 tumours, a CR was achieved in 8 of the 14 patients $(57 \%)$ compared 4 out of 16 patients $(25 \%)$ in the group with $T 2$ tumours $(p=0.07)$.

\section{Loco-regional tumour control}

The minimal follow up was 36 months in all patients. The median loco-regional control time was 30.7 months (95\%-ci: 15.6-45.8 months). There were no regional recurrences. Loco-regional control was $83 \%$ after 1 year, $67 \%$ after 2 years and $40 \%$ after 3 years. In the group of patients with a complete response after radiotherapy, there were no locoregional recurrences. In the group without a complete response, the median loco-regional control time was 17.7 months (95-ci: 8.2-27.2 months), which was significantly worse 
compared to the complete responders $(p=0.002)$. After 3 years, all patients without $C R$ had developed a local recurrence.

\section{Distant metastases}

Distant metastases developed in two patients after 6.2 and 8.9 months respectively.

\section{Overall survival}

The overall survival (OS) of all patients was $69 \%$ at 1 year, $37 \%$ at 2 years and $16 \%$ at 3 years. The median OS was 19.1 months (95\%-ci: 15.3-22.9 months). The median OS in the peripherally located tumours was 19.5 months (95-ci: $13.7-25.3$ months) compared to 8.1 months (95-ci: $0.6-15.6$ months) for those with a centrally localised tumour $(p<0.001)$ (Figure 1). No difference was observed between T1 and T2 tumours.

Figure 1: Survival stratified by site.

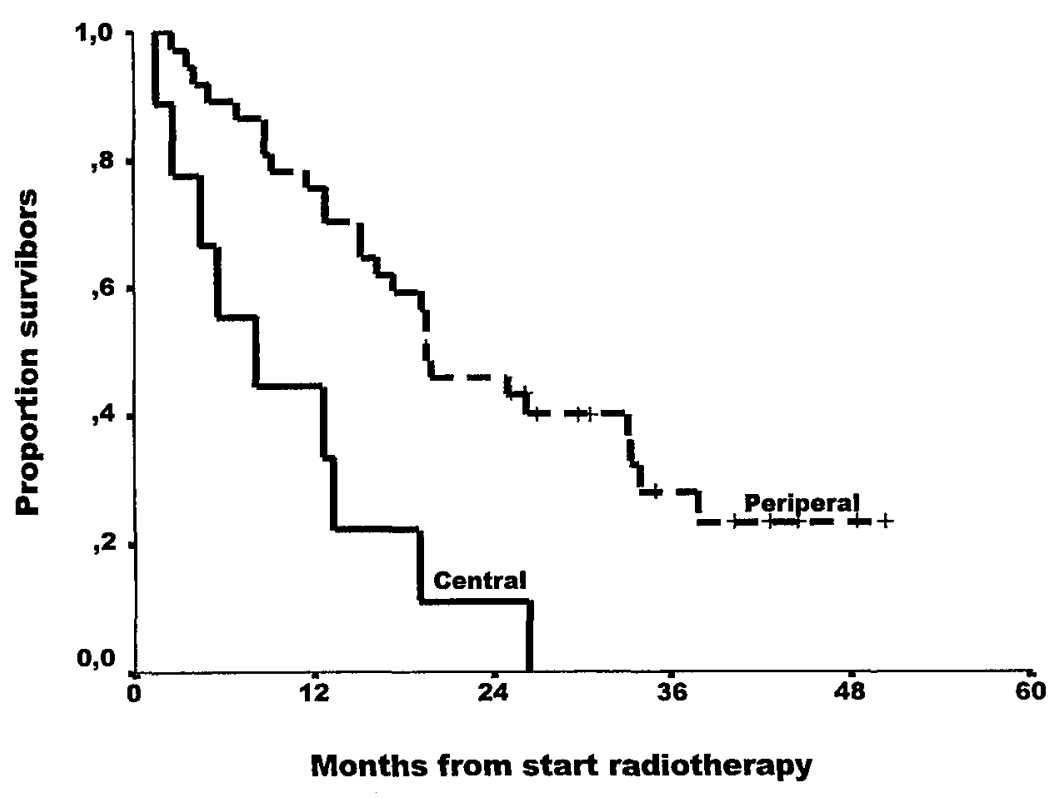


Table 2: Pre-treatment symptoms

\begin{tabular}{|c|c|c|}
\hline Symptom or functioning scale & $\%$ of patients & $\begin{array}{c}\text { Mean score in all } \\
\text { patients (SD) }\end{array}$ \\
\hline \multicolumn{3}{|l|}{ Symptoms } \\
\hline Cough & 86 & $41.9(28.4)$ \\
\hline Dyspnea & 86 & $33.2(26.0)$ \\
\hline Fatigue & 80 & $32.9(28.5)$ \\
\hline Pain & 46 & $8.6(20.4)$ \\
\hline Insomnia & 46 & $23.8(31.9)$ \\
\hline Pain arm/shoulder & 23 & $13.3(28.2)$ \\
\hline Haemoptysis & 23 & $10.5(21.0)$ \\
\hline Appetite loss & 20 & $8.6(20.4)$ \\
\hline Constipation & 20 & $7.6(16.3)$ \\
\hline Pain chest wall & $\uparrow 7$ & $8.6(20.4)$ \\
\hline Dysphagia & 14 & $7.6(21.5)$ \\
\hline Nausea and vomiting & 11 & $2.9(9.5)$ \\
\hline \multicolumn{3}{|c|}{ Functioning scales \& global quality of life } \\
\hline Physical functioning & - & $56.1(25.8)$ \\
\hline Role functioning & - & $75.7(32.9)$ \\
\hline Emotional functioning & - & $71.7(21.5)$ \\
\hline Cognitive functioning & - & $83.8(18.7)$ \\
\hline Social functioning & - & $85.7(22.2)$ \\
\hline Global quality of life & - & $56.7(22.2)$ \\
\hline
\end{tabular}

Compliance of quality of lifeA total number of 35 patients were entered in the study after informed consent and all these patients filled out a baseline questionnaire. The compliance calculated over the patients who were still alive was $100 \%$ at the assessments made during radiotherapy up to 3 months after radiotherapy. The compliance was $>90 \%$ at all the other time points. 
Figure 2: Course of the mean scores for (1a) respiratory and (1b) general symptoms and the (1c) functioning and global quality of life scales.
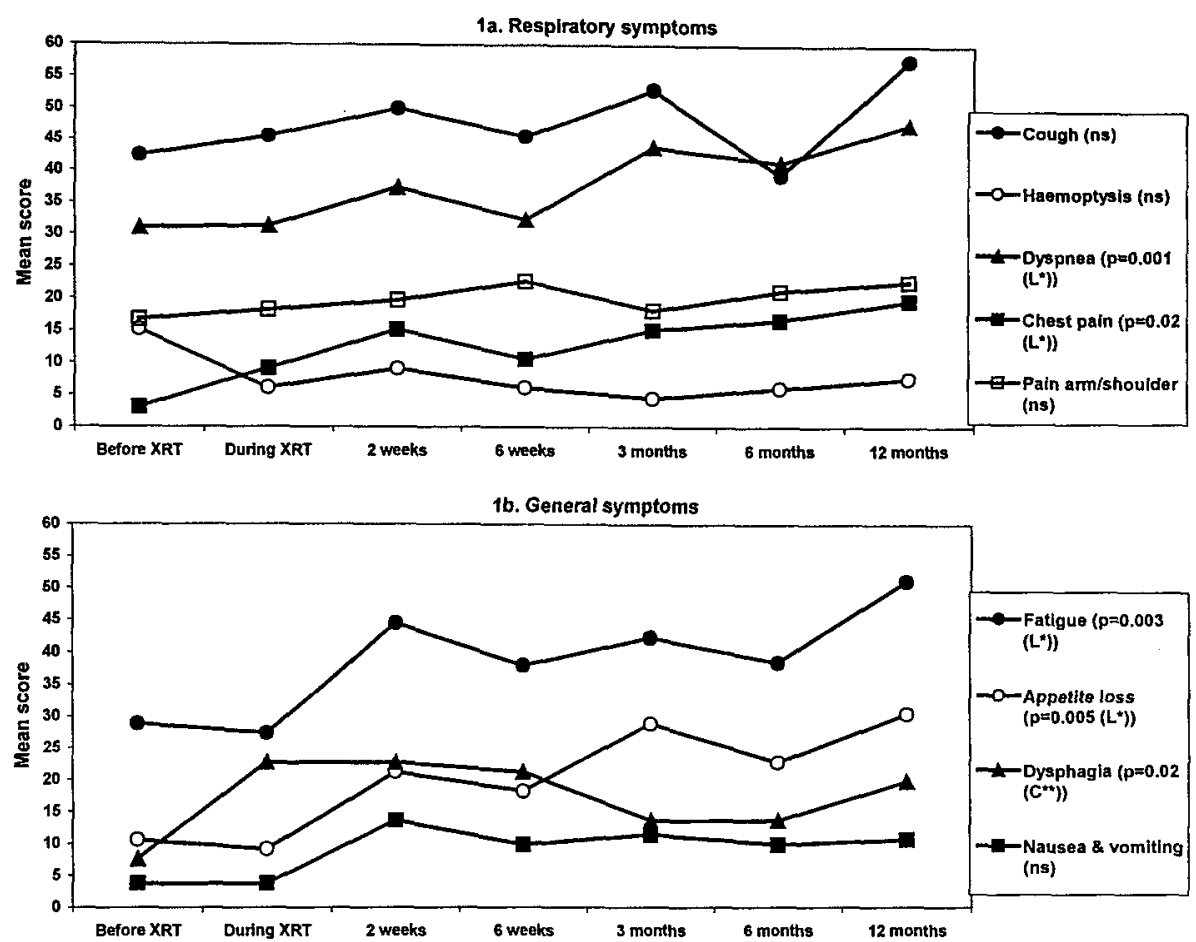

1c. Functloning and global QoL scales

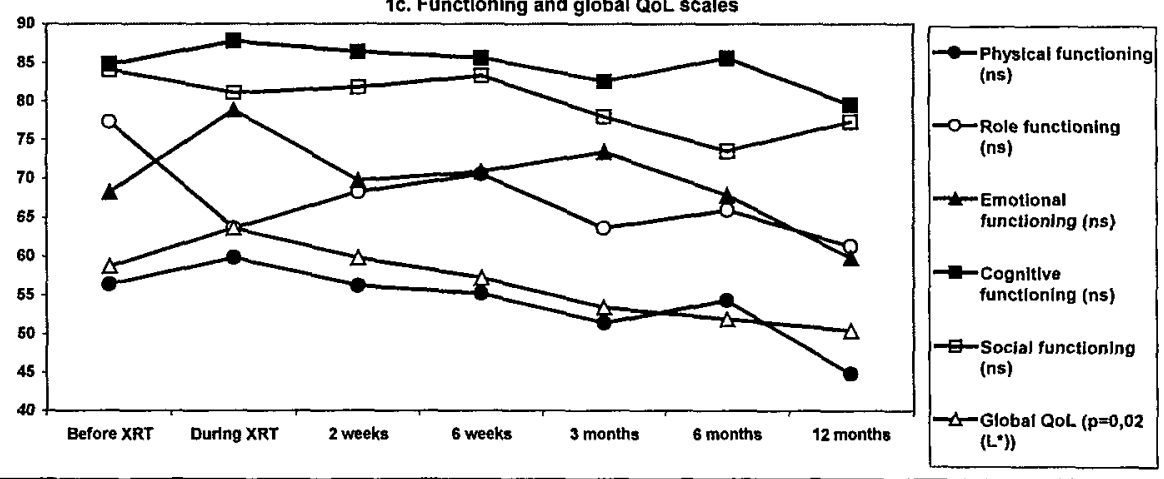

Note: The p-values refer to the comparison of the mean values observed before, during and after radiotherapy ( 2 weeks, 6 weeks and 3,6 and 12 months) calculated using the general linear model for repeated measurements. * $L=$ linear association

${ }^{\star \star} \mathrm{C}=$ cubic association 


\section{Baseline symptoms and QoL}

Cough and dyspnea were the most frequently reported respiratory symptoms, both present in $86 \%$ of the patients (Table 2). The most frequently reported general symptom was fatigue, which was present in $80 \%$ of the cases. The mean baseline values of the functioning scales varied from 56.1 for physical functioning to 85.7 for social functioning. The mean baseline score for global QoL was 56.7 .

\section{Changes in mean symptom and QoL scores over time}

Regarding the respiratory symptoms, a significant gradual increase was observed for dyspnea $(p=0.001)$ and chest pain $(p=0.02)$ (Figure $2 a)$. For dyspnea, this increase started during radiotherapy and gradually increased when time passed. For chest pain, the strongest increase was observed during and immediately after radiotherapy. The mean score for haemoptysis dropped during radiotherapy and remained at the same level up to 12 months.

For the general symptoms, the changes of the mean scores are presented in Figure $2 \mathrm{~b}$. A significant gradual increase was observed for fatigue $(p=0.003)$ and appetite loss $(p=0.005)$. For both symptoms, this worsening started 2 weeks after radiotherapy and gradually progressed up to 12 months. Dysphagia increased during radiotherapy and improved after radiotherapy but never returned to the baseline level. Significantly higher levels of dysphagia $(p=0.03$ ) were observed in the group of patients treated with locoregional irradiation compared to those only irradiated on the primary site (Figure 3 ). Between these two groups, no significant differences were observed regarding the other respiratory and general symptoms. For nausea and vomiting, no significant changes were observed, although there was a slight increase after radiotherapy.

The changes in the mean score for the functioning scales and global QoL scale are presented in Figure 2c. For all the functioning scales, a gradual decrease was observed. In 12 months, the mean value for physical functioning decreased with 11.6 points, 16.0 points for role functioning, 8.4 points for emotional functioning, 5.3 points for cognitive functioning and 6.8 points for social functioning. These changes were not statistically significant. A significant gradual deterioration was only observed for global $Q \mathrm{LL}(p=0.02)$. The mean value of global QoL dropped with 8.4 points in 12 months. No significant 
Figure 3: Mean score for dysphagia stratified by target volume.

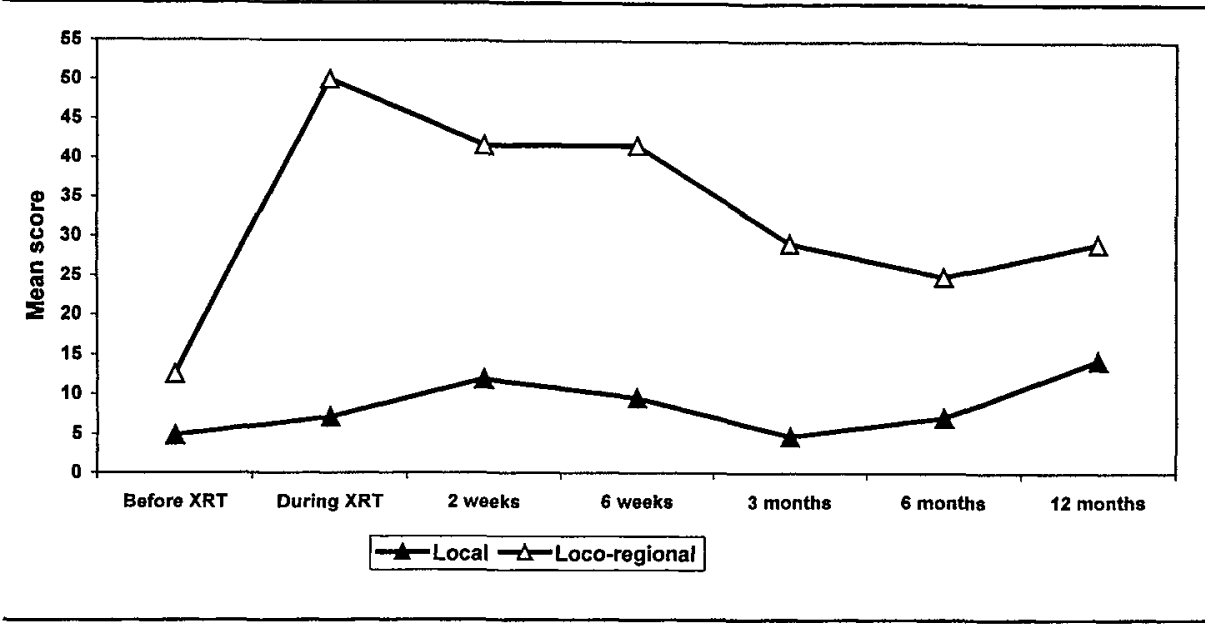

differences were found between locally treated patients and patients electively treated on the regional lymph node areas.

\section{Symptom and quality of life response}

Improvement of cough, the most frequently reported respiratory symptom, was achieved in only four of the 28 patients (14\%) who suffered from cough before treatment (Table 3). The total response rate for cough was $52 \%$, mainly due to the relatively large proportion of patients classified as having control. A comparable response pattern was observed for dyspnea. The total response rate for dyspnea was $36 \%$. Progression of cough and dyspnea was observed in $18 \%$ of the cases. For the other respiratory symptoms, the response rate for haemoptysis was $84 \%, 58 \%$ for chest pain and $61 \%$ for arm/shoulder pain. For fatigue, the response rate was $36 \%$, while $27 \%$ of the patients deteriorated.

The response rates for the functioning scales varied from $47 \%$ for social functioning to $61 \%$ for emotional functioning (Table 4). However, deteriorating functioning was observed in a considerable proportion of patients ranging from $24 \%$ for cognitive and role functioning to $33 \%$ for physical functioning. The response rate for global QoL was $21 \%$, while $42 \%$ of the patients experienced worsening of global QoL. 
Table 3: Response classification for general and respiratory symptoms ( $n=35)$.

\begin{tabular}{lcrrrrrr}
\hline Symptom & $\begin{array}{c}\text { Inadequate } \\
\text { follow up } \\
\text { data }\end{array}$ & Improvement & Control & Prevention & $\begin{array}{c}\text { Dead without } \\
\text { palliation }\end{array}$ & Response rate & Worse \\
\hline Cough & 2 & $4 / 28(14 \%)$ & $11 / 19(58 \%)$ & $2 / 5(40 \%)$ & $2 / 33(6 \%)$ & $17 / 33(52 \%)$ & $6 / 33(18 \%)$ \\
Haemoptysis & 3 & $5 / 8(63 \%)$ & $1 / 5(20 \%)$ & $21 / 24(88 \%)$ & $1 / 32(3 \%)$ & $27 / 32(84 \%)$ & $2 / 32(6 \%)$ \\
Dyspneu & 2 & $3 / 28(11 \%)$ & $7 / 15(47 \%)$ & $2 / 5(40 \%)$ & $2 / 33(6 \%)$ & $12 / 33(36 \%)$ & $6 / 33(18 \%)$ \\
Pain arm/shoulder & 4 & $5 / 8(63 \%)$ & $1 / 4(25 \%)$ & $14 / 23(61 \%)$ & $0 / 31(0 \%)$ & $19 / 31(36 \%)$ & $9 / 31(29 \%)$ \\
Chest pain & 4 & $1 / 4(25 \%)$ & $0 / 3(0 \%)$ & $17 / 27(61 \%)$ & $0 / 31(0 \%)$ & $18 / 31(58 \%)$ & $9 / 31(29 \%)$ \\
Fatigue & 2 & $3 / 26(12 \%)$ & $7 / 14(50 \%)$ & $2 / 7(29 \%)$ & $2 / 33(6 \%)$ & $12 / 33(36 \%)$ & $9 / 33(27 \%)$ \\
\hline
\end{tabular}

* Note: the number of patients with inadequate follow up data may differ between scales because of the definition of 'inadequate follow up' (see appendix 1)

Table 4: Response classification for functioning and global quality of life $(n=35)$.

\begin{tabular}{lcrrrrrr}
\hline Symptom & $\begin{array}{c}\text { Inadequate } \\
\text { follow up } \\
\text { data }\end{array}$ & Improvement & Control & Prevention & $\begin{array}{c}\text { Dead without } \\
\text { palliation }\end{array}$ & Response rate & Worse \\
\hline PF & 28 & $8 / 33(24 \%)$ & $8 / 11(73 \%)$ & $2 / 9(22 \%)$ & $2 / 33(6 \%)$ & $18 / 33(55 \%)$ & $11 / 33(33 \%)$ \\
RF & 29 & $5 / 33(15 \%)$ & no patients & $11 / 20(55 \%)$ & $2 / 33(6 \%)$ & $16 / 33(48 \%)$ & $8 / 33(24 \%)$ \\
EF & 33 & $16 / 33(48 \%)$ & $1 / 11(9 \%)$ & $3 / 12(25 \%)$ & $2 / 33(6 \%)$ & $20 / 33(61 \%)$ & $9 / 33(27 \%)$ \\
CF & 34 & $8 / 33(24 \%)$ & $0 / 6(0 \%)$ & $10 / 23(43 \%)$ & $2 / 33(6 \%)$ & $18 / 33(55 \%)$ & $8 / 33(24 \%)$ \\
SF & 3 & $6 / 32(19 \%)$ & $8 / 24(33 \%)$ & $1 / 4(25 \%)$ & $1 / 21(3 \%)$ & $15 / 32(47 \%)$ & $9 / 32(28 \%)$ \\
Global QoL & 25 & $7 / 33(21 \%)$ & $0 / 6(0 \%)$ & $0 / 8(0 \%)$ & $0 / 33(0 \%)$ & $7 / 33(21 \%)$ & $14 / 33(42 \%)$ \\
\hline
\end{tabular}

* Note: the number of patients with inadequate follow up data may differ between scales because of the definition of 'inadequate follow up' (see appendix 2).

\section{Radiation-induced pulmonary changes}

The incidence as well as the severity of radiological changes induced by radiotherapy increased with time. After 12 months, patchy, dense and severe changes were observed in 12 of the 20 patients at risk $(60 \%)$. A clear difference was observed between the group of patients treated locally as compared to those in which the target volume included the hilar and mediastinal lymph node areas (Figure 4).

\section{ㅁiscussion}

To our knowledge, this is the first prospective study in patients treated with curative radiotherapy in which QoL was one of the endpoints. The only study that reported on QoL 
Figure 4: Radiation-induced pulmonary changes stratified by target volume.

Local irradiation

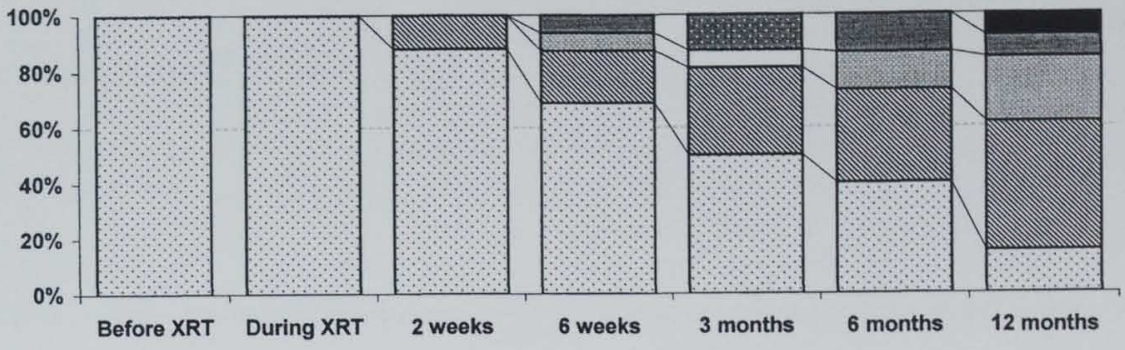

Loco-regional irradiation

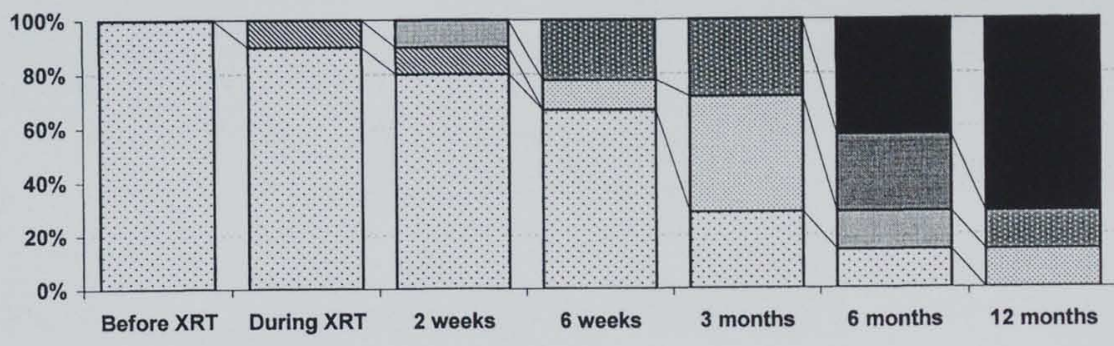

$\square$ No changes $\mathbb{Q}$ Minimal $\square$ Patchy (⿴囗十) Dense $\mathbf{\square}$ Severe

issues in curatively treated NSCLC was carried out by Dales and co-workers ${ }^{19}$. They investigated QoL in 92 patients with lung cancer and in 25 patients without lung cancer. Both groups underwent a thoracotomy. In those with cancer, QoL deteriorated postoperatively but returned to baseline levels 6 to 9 months after surgery. In those without cancer, no deterioration of QoL was noted after surgery. They concluded that QoL in surgically treated lung cancer patients resulted in a transient worsening of QoL in the first three months after surgery, but that improvement back to baseline can be expected.

The 3 -year overall survival was $19 \%$ which is in accordance with other studies concerning stage I NSCLC treated with curative radiotherapy, in which 3 year-survival rates have been reported varying from $18 \%$ to $42 \%{ }^{2,6-8,10-12}$. Although, these survival rates are more favourable as compared to those observed in locally advanced and metastatic NSCLC ${ }^{20-}$ 
${ }^{23}$ the overall survival remains limited in the majority of patients. Therefore, QoL issues remain of considerable interest, also in this category of patients.

About the respiratory symptoms, a gradual increase was observed for dyspnea and chest pain. Comparable results were observed for fatigue and appetite loss. Subsequently, a significant gradual deterioration for global QoL was observed as well. These observations were not what was expected. In the first 12 months after radiotherapy, loco-regional recurrences were observed in only four and distant metastases in only two patients. Therefore, it is unlikely that this gradual deterioration is determined by tumour progression itself. In the present study, all patients but one were inoperable due to pre-existing comorbidity. In the majority of these patients, lung function was considered inadequate for surgical treatment due to chronic obstructive pulmonary disease. Data collected on large populations with chronic airway obstruction demonstrate a slow deterioration in ventilatory function and subsequent respiratory symptoms ${ }^{24-26}$. The natural history of chronic obstructive pulmonary disease could therefore contribute to the worsening of dyspnea and general symptoms including fatigue as observed in the present study. Late sequelae of radiotherapy include radiation pneumonitis and pulmonary fibrosis. In the present study, radiation-induced pulmonary changes increased when time past. At 12 months, radiationinduced patchy, dense or severe changes were observed in the majority of cases still alive. These changes may also contribute to an acceleration of the gradual increase of dyspnea of patients with COPD and subsequent worsening of general symptoms and deterioration of QoL.

During radiotherapy and 2 weeks after radiotherapy, higher levels of chest pain were observed. After a small drop at 6 weeks, chest pain gradually increased with time. The increase observed during and shortly after radiotherapy could be due to a transient pleural irritation induced by radiotherapy. The increase of chest pain after 3 months could be connected to the radiation-induced pulmonary changes with secondary pleural irritation. This is supported by the observation that in a number of patients with severe pulmonary changes on chest radiograph, pleural effusion developed.

For the functioning scales, deterioration was observed varying from 5.3 points for cognitive functioning to 16.0 points for role functioning. Osoba and co-workers investigated the significance to patients of changes in health-related $\mathrm{QoL}^{27}$. A change in scores between 5 to 10 points were indicated by the patients as 'a little' change either for better or for worse. 
A 'moderate' change indicated by the patient corresponded with a change in scores between 10 to 20 points and 'very much' change corresponded with a change in scores greater than 20. Although the changes over time for the functioning scales in the present study were not statistically significant, which may be due to the relatively small sample size, they still may be of interest.

At present, there is discussion as to whether elective irradiation of the hilar and mediastinal lymph nodes in clinically NO disease is indicated. In the present study, none of the 20 locally treated patients developed a regional recurrence after a minimal follow up period of three years. It has to be taken into account that almost all locally treated patients had peripherally located tumours. In two other studies reporting on curative radiotherapy in stage I NSCLC, regional recurrences were observed in $2 \%{ }^{11}$ and $8 \%{ }^{8}$.

As expected, the levels of dysphagia were significantly higher in the patients treated with loco-regional irradiation as compared to in those who were locally treated. A striking finding was that the difference in the level of dysphagia remained present even after 12 months. After loco-regional irradiation, the incidence and severity of radiological changes assessed with chest radiograph was higher compared to those observed after local irradiation. Although the omission of the elective nodal areas out of the target volume resulted in a decrease of dysphagia and radiation-induced pulmonary changes, no differences were observed regarding other symptoms, functioning and global QoL, which could also be due to the relatively small sample size.

In conclusion, after curative radiotherapy for stage I medically inoperable NSCLC, a gradual increase of dyspnea and chest pain is observed together with a significant deterioration of global QoL, possibly due to pre-existing, slowly progressive chronic obstructive pulmonary disease and radiation-induced pulmonary changes. Taking into account the low incidence of regional recurrences after local irradiation, the higher incidence and severity of radiation-induced changes and the higher levels of dysphagia persisting up to 12 months, local irradiation should be considered in small peripherally located tumours. 


\section{$\square$ References}

1. In Hermanek P and Sobin LH (Ed) In: TNM Atlas: Classification of malignant tumours; Springer-Verlag, Berlin 1992: pp 75-84.

2. Coy, $P$ and Kennelly, GM The role of curative radiotherapy in the treatment of lung cancer. Cancer 1980; 45: 698702.

3. Dosoretz, DE, Galmarini, D, Rubenstein, et al. Local control in medically inoperable lung cancer: an analysis of its importance in outcome and factors determining the probability of tumor eradication. J Radiat Oncol Biol Phys 1993; 27: 507-516.

4. Gauden, $S$, Ramsay, $J$ and Tripciony, L.. The curative treatment by radiotherapy alone of stage I non-small cell lung cancer. Chest 1995; 108: 1278-1282.

5. Graham, RH, Gebski, VJ and Langlands, AO. Radical radiotherapy for early nonsmall cell lung cancer. Int J Radiat Oncol Biol Phys 1995; 31: 261-266.

6. Haffty, BG, Goldberg, NB., Gerstley, et al. Results of radical radiation therapy in clinical stage I, technically operable non-small cell lung cancer. Int J Radiat Oncol Biol Phys 1988; 15: 69-73.

7. Kaskowitz, L, Graham, MM, Emami, B, Halverson, KJ and Rush, C. Radiation therapy alone for stage 1 non-small cell lung cancer. J Radiat Oncol Biol Phys 1993; 27: 517-523.

8. Krol, ADG, Aussems, P, Noordijk, FM, Hermans, J. and Leer, JWH. Local irradiation alone for peripheral stage I lung cancer: could we omit the elective regional nodal irradiation? Int J Radiat Oncol Biol Phys 1996; 34: 297-302.

9. Noordijk, EM, v.d. Poest Clement, F, Hermans, J, Wever, AMJ and Leer, JWH. Radiotherapy as an alternative to surgery in elderly patients with resectable lung cancer. Radiot Oncol 1988; 13: 83-89.

10. Sandler, HM, Curran Jr WR and Tunisi III, AT. The influence of tumor size and pre-treatment staging on outcome following radiation therapy alone for stage I non-small cell lung cancer. Radiat Oncol Biol Phys 1990; 19: 9-13.

11. Slotman, BJ., Anthonisse IE, and Njo, KH. Limited field irradiation in early stage (T1-2NO) non-small cell lung cancer. Radiot Oncol 1996; 41: 41-44.

12. Talton, $B M$, Constable, $W C$ and Kersh, CR. Curative radiotherapy in non-small cell carcinoma of the lung. Int $J$ Radiat Oncol Biol Phys 1990; 19: 15-21.

13. Zhang, $\mathrm{HX}$, Yin, WB, Zhang, et al. Curative radiotherapy of early operable non-small cell lung cancer. Radiot Oncol 1989; 14: 89-94.

14. Aaronson NK, Ahmedzai S, Bergman B, et al. The European Organization for Research and Treatment of Cancer QLQ-C30: a quality of life instrument for use in international clinical trials in oncology. J Natl Cancer Inst 1993; 85: 36576.

15. Bergman B, Aaronson NK, Ahmedzai S, et al. The EORTC QLQ-LC13: A modular supplement to the EORTC Core Quality of Life Questionnaire (QLQ-C30) for use in clinical trials in oncology. Eur J Cancer 1994; 30A: 635-642.

16. Pavy JJ, Denekamp J, Letschert J, et al. EORTC Late Effects Working Group. Late effects toxicity scoring: the SOMA scale. Radiother Oncol 1995; 35:11-15.

17. Langendijk JA, ten Velde GPM, Aaronson NK, et al. Quality of life after palliative radiotherapy in non-small cell lung cancer: a prospective study. Int J Radiat Oncol Biol Phys 2000; 47: 149-155.

18. Langendijk JA, Lamers RJS, ten Velde GPM, et al. Is the chest radiograph a reliable tool in the assessment of tumor response after radiotherapy in nonsmall cell lung carcinoma. Int J Radiat Oncol Biol Phys 1998; 41: 1037-1045.

19. Dales RE, Belanger R, Shamji FM, et al. Quality of life following thoracotomy for lung cancer. J Clin Epidemiol 46 : 1443-1449, 1994. 


\section{Chapter 8}

20. Perez CA, Stanley K, Grundy G, et al. Impact of irradiation technique and tumor extent in tumor control of patients with unresectable non-oat cell carcinoma of the lung. Cancer 1982; 50: 1091-1099.

21. Bulzebruck, $H$, Bopp $R$, Drings $P$, et al. New aspects in the staging of lung cancer. Prospective validation of the International Union Against Cancer TNM Classification. Cancer 1992; 70: 1102-1110.

22. Cox JD, Azarnia N, Byhardt RW, et al. N2 (clinical) non-small cell carcinoma of the lung: Prospective trials of radiation therapy with total doses of 60 Gy by the Radiation Therapy Oncology Group. Int J Radiat Oncol Biol Phys 1991; $20: 7$ 12.

23. Curran WJ, Stafford PM. Lack of apparent differences in outcome between clinically staged IItA and IIIB non-small cell lung carcinoma treated with radiotherapy. J Clin Oncol 1990; 8: 409-415.

24. Guell $R$, Casan $P$, Sangenis $M$, et al. The Spanish translation and evaluation of a quality of life questionnaire in patients with chronic obstructive pulmonary disease. Arch Bronconeumol 1995; 31:202-210.

25. Lange P, Pamer J, Vestbo et al. A 15-year follow-up study of ventilatory function in adults with asthma. $\mathrm{N}$ Engl $\mathrm{J}$ Med 1998; 22: 1194-1200.

26. Scharloo M, Kaptein AA, Weinman JA, et al. Physical and psychological correlates of functioning in patients with chronic obstructive pulmonary disease. J Asthma 2000; 37: 17-29.

27. Osoba, D, Rodrigues $\mathrm{G}$, Myles $\mathrm{J}$, et al. Interpreting the significance of changes in quality of life scores. J Clin Oncol 1998; 16: 139-144. 


\section{Appendix 1}

\section{Response criteria for general and respiratory symptoms}

\section{'Response' is defined as:}

- Baseline score 'moderate or severe', with improvement to 'mild' or 'nil' $\dagger=$ 'improvement'.

- Baseline score 'mild', with improvement to 'nil' $\dagger=$ 'improvement'.

- Baseline score 'mild', with 'mild' $t=$ 'control'.

- Baseline score 'nil', with 'nil' $\dagger=$ = 'prevention'.

\section{'No response' is defined as:}

- Baseline score 'moderate or severe', without improvement to 'mild or nil' $t=$ 'no change'

- Baseline score 'moderate or severe' and dead before first assessment after the end of radiotherapy = 'dead without palliation'.

- Baseline score 'moderate or severe' without improvement to 'mild or nil' on the first assessment and dead before the second assessment after the end of radiotherapy = 'dead without palliation'.

- Baseline score 'mild', without improvement to 'nil' and without deterioration to 'moderate or severe' $\dagger=$ 'no change'.

- Baseline score 'mild' and dead before first assessment after the end of radiotherapy = 'dead without palliation'.

- Baseline score 'mild', without improvement to 'nil' on the first assessment and dead before first assessment after the end of radiotherapy $=$ 'dead without palliation'.

- Baseline score 'nil', without 'nil' and without deterioration to 'mild, moderate or severe' $t=$ 'no change'.

- Baseline score 'nil', with deterioration to 'mild, moderate or severe' on the first assessment and dead before the second assessment after the end of radiotherapy = 'dead without palliation'.

'Progression' is defined as:

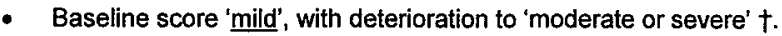

- Baseline score 'nil', with 'mild, moderate or severe' $t$.

\section{'Not evaluable' is defined as:}

- Baseline score 'moderate or severe', with improvement to 'nil or mild' on the first assessments and dead before the second assessment after the end of radiotherapy.

- Baseline score 'mild', with improvement to 'nil' on the first assessment and dead before the second assessment after the end of radiotherapy.

- Baseline score 'nil' and dead before the first assessment after the end of radiotherapy.

- Baseline score 'nil', with 'nil' at the first assessment and dead before the second assessment after the end of radiotherapy.

$t=$ on at least two consecutive assessments in the first three months after the end of radiotherapy 


\section{Appendix 2}

\section{Response criteria for functioning scales and global quality of life}

\section{'Response' is defined as:}

- Baseline score '0-59', with improvement of at least 5 points $\dagger$ to a minimal value of $40=$ 'improvement'.

- Baseline score ' $60-79$ ', with improvement of at least 5 points $\dagger=$ 'improvement'

- Baseline score ' $60-79$ ', with no change (i.e. $<5$ points) $t=$ 'control'

- Baseline score ' $80-100$ ', with improvement of at least 5 points $\dagger=$ 'improvement'

- Baseline score ' $\underline{80-100}$ ', with no change (i.e. $<5$ points) $\dagger=$ 'prevention'

\section{'No response' is defined as:}

- Baseline score ' $0-59$ ', with no change (i.e. $<5$ points) $\dagger=$ 'no change'

- Baseline score ' $0-59$ ' and dead before first assessment after the end of radiotherapy = 'dead without palliation'.

- Baseline score ' $0-59$ ' without improvement of at least 5 points to a minimal value of 40 on the first assessment and dead before the second assessment after the end of radiotherapy = 'dead without palliation'.

- Baseline score '60-79' and dead before first assessment after the end of radiotherapy = 'dead without palliation'.

- Baseline score ' $60-79$ ' without improvement of at least 5 points on the first assessment and dead before the second assessment after the end of radiotherapy = 'dead without palliation'.

- Baseline score ' $\underline{80-100}$ ', and dead before first assessment after the end of radiotherapy = 'dead without palliation'.

- Baseline score ' $80-100$ ' without improvement of at least 5 points on the first assessment and dead before the second assessment after the end of radiotherapy = 'dead without palliation'.

'Progression' is defined as:

- Baseline score '0-59', with a decrease of at least 5 points $\dagger$

- Baseline score ' $60-79$ ', with a decrease of at least 5 points $f$

- Baseline score ' $80-100$ ', with a decrease of at least 5 points $\dagger$

'Not evaluable' is defined as:

- Baseline score ' $\underline{0.59}$, with improvement of at least 5 points to a minimal value of 40 on the first assessments and dead before the second assessment after the end of radiotherapy.

- Baseline score ' $\underline{60-79}$ ', with improvement of at least 5 points on the first assessment and dead before the second assessment after the end of radiotherapy.

- Baseline score ' $80-100$ ', with improvement of at least 5 points on the first assessment and dead before the second assessment after the end of radiotherapy.

$\dagger=$ on at least two consecutive assessments in the first three months after the end of radiotherapy 
Chapter 9

Massive haemoptysis after radiotherapy in inoperable non-small cell lung carcinoma: is endobronchial brachytherapy really a risk factor?

\author{
Hans Langendijk \\ Martin Tjwa \\ Jos de Jong \\ Guul ten Velde \\ Miel Wouters
}

Published in:

Radiotherapy and Oncology 1998; 49: 175-183. 


\section{a Abstract}

Background and purpose: This retrospective study was conducted to investigate whether endobronchial brachytherapy (EBB) is a risk factor for massive haemoptysis in patients primarily treated by a combination of EBB and external irradiation (XRT) for NSCLC.

Material and methods: The records of 938 patients with inoperable NSCLC who were treated with XRT and/or EBB were reviewed. The patients were divided into 5 groups: Group XRT: treated by XRT alone ( $n=421$ ), group XRTelig: treated by XRT but eligible for EBB $(n=419)$, group XRTEBB: primarily treated with $E B B+X R T(n=62)$, group EBBrec: treated by EBB for recurrence after XRT $(n=23)$ and group EBB: treated by EBB alone $(n=13)$. EBB was delivered using HDR. Patients with bronchoscopy-proven endobronchial tumour in the proximal airways, i.e. the trachea, the main bronchus or lobar bronchus were considered eligible for EBB.

Results: 101 out of 938 patients (10.8\%) died from massive haemoptysis. The incidence in group XRT was $4.3 \%, 13.1 \%$ in group XRTelig and $25.4 \%$ in group XRTEBB. The differences between group XRT and XRTelig as well as between group XRTelig and $X R T E B B$ were statistically significant $(p<0.01)$. The incidence of massive haemoptysis depended significantly on the fraction size of brachytherapy. When 2 fraction of 7.5 Gy or a single fraction of $10 \mathrm{~Gy}$ were used, $11.1 \%$ of the patients died from massive haemoptysis. However, when a single dose of $15 \mathrm{~Gy}$ was used, $47.8 \%$ died from massive haemoptysis. In the multivariate analysis, a single dose of $15 \mathrm{~Gy}$ EBB was the most important prognostic factor for massive haemoptysis.

Conclusion: $\mathrm{XRT+EBB}$ as primary treatment for NSCLC does not lead to a higher risk of massive haemoptysis as compared to XRT alone when fraction sizes for EBB are used of $7.5 \mathrm{~Gy}$ or $10 \mathrm{~Gy}$. However, the risk of massive haemoptysis increases dramatically when a fraction size of $15 \mathrm{~Gy}$ is used. 


\section{口 Introduction}

Reports regarding massive haemoptysis after external beam radiotherapy are scarce. However, massive haemoptysis after endobronchial brachytherapy (EBB) is a frequently described fatal complication in non-small cell lung carcinoma (NSCLC). A number of studies reported on the results of endobronchial brachytherapy in bronchial carcinoma and in these series incidence rates for massive haemoptysis are ranging from 0 to $50 \%^{1-15,17-25}$. The question arises whether EBB itself plays a causative role in the development of this fatal complication. However, the high incidence of massive haemoptysis after EBB may be explained by selection of patients with centrally localised tumours in the proximal airways in the vicinity of the great vessels and thus at risk for massive haemoptysis. In the past 10 years, the treatment policy of patients with inoperable centrally localised tumours, referred for radiotherapy to our department has been changing. When HDR-afterloading equipment became available, this category of patients was more frequently treated with a combination of external irradiation (XRT) and EBB. This allowed us to compare the incidence of massive haemoptysis in subsets of patients treated with XRT alone and XRT in combination with EBB.

The objectives of this retrospective study were:

(1) to assess pre-treatment risk factors for massive haemoptysis,

(2) to investigate if patients treated with (XRT), but who were eligible for EBB were at risk for massive haemoptysis,

(3) to investigate whether massive haemoptysis was more frequently observed in patients actually treated with a combination of EBB and XRT as compared to patients eligible for EBB but treated with XRT alone and finally,

(4) to investigate the role of treatment-related factors, such as fraction size of EBB.

\section{Material and methods}

\section{Patients}

Patients who were referred for radiotherapy to the RTIL in the period of 1987 to 1996 with inoperable NSCLC were included. The diagnosis had to be confirmed by cytology and/or 
histology and patients had to be treated with XRT and/or EBB. Initially, a total number of 966 patients fulfilled these inclusion criteria. The endpoint of this study was whether the patient died of massive haemoptysis or not. Massive haemoptysis was defined as an

Table 1: Patients characteristics

\begin{tabular}{|c|c|c|c|}
\hline Variable & & $\begin{array}{c}\text { Total } \\
\text { number }\end{array}$ & Percentage \\
\hline \multirow[t]{2}{*}{ Sex } & Female & 111 & $11.8 \%$ \\
\hline & Male & 827 & $88.2 \%$ \\
\hline \multirow[t]{2}{*}{ Age } & $0-70$ years & 572 & $61.0 \%$ \\
\hline & $>70$ years & 366 & $39.0 \%$ \\
\hline \multirow[t]{5}{*}{ T-classification } & $\mathrm{T} 1$ & 93 & $9.9 \%$ \\
\hline & $\mathrm{T} 2$ & 410 & $43.8 \%$ \\
\hline & T3 & 199 & $21.3 \%$ \\
\hline & $\mathrm{T} 4$ & 234 & $25.0 \%$ \\
\hline & unknown & 2 & \\
\hline \multirow[t]{5}{*}{$\mathrm{N}$-classification } & No & 362 & $40.2 \%$ \\
\hline & N1 & 15 & $1.7 \%$ \\
\hline & N2 & 404 & $44.9 \%$ \\
\hline & N3 & 119 & $13.2 \%$ \\
\hline & unknown & 37 & \\
\hline \multirow[t]{6}{*}{ Performance } & WHO $O$ & 318 & $34.1 \%$ \\
\hline & WHO 1 & 407 & $43.7 \%$ \\
\hline & WHO 2 & 128 & $13.7 \%$ \\
\hline & WHO 3 & 74 & $7.9 \%$ \\
\hline & WHO 4 & 5 & $0.5 \%$ \\
\hline & unknown & 6 & \\
\hline \multirow[t]{3}{*}{ Weight loss } & $0-10 \%$ & 690 & $76.5 \%$ \\
\hline & $>10 \%$ & 212 & $23.5 \%$ \\
\hline & unknown & 36 & \\
\hline \multirow[t]{4}{*}{ Histology } & Squamous cell carcinoma $(\mathrm{SqCC})$ & 601 & $64.1 \%$ \\
\hline & Adenocarcinoma & 123 & $13.1 \%$ \\
\hline & Large cell carcinoma & 194 & $20.7 \%$ \\
\hline & Others & 20 & $2.1 \%$ \\
\hline
\end{tabular}


unmistakable event characterised by the coughing up of massive amounts of blood or clot followed within minutes by asphyxiation or death ${ }^{2}$. After reviewing the medical records retrospectively, it remained unclear in 311 patients if the patient died from massive haemoptysis. In these cases, the general practitioner or other medical specialists were interviewed by telephone. After this procedure, 28 patients (2.9\%) were excluded, because in these subjects it remained unclear if these patients died from massive haemoptysis. The remaining 938 patients were used for this analysis. There were 827 men $(88.2 \%)$ and 111 women $(11.8 \%)$ with a median age of 68 years, ranging from 36 to 88 years. The characteristics of the patients in this study are listed in table $\mathrm{I}$.

\section{Treatment groups}

Patients were divided into 5 treatment groups. The first group (group XRT) consisted of 421 patients (44.9\%), treated with XRT alone. The second group (group XRTelig) consisted of 419 patients $(44.7 \%)$, treated with XRT alone, but who would have been eligible for EBB. Patients with bronchoscopy-proven endobronchial tumour in the proximal airways, i.e. the trachea, the main bronchus or lobar bronchus were eligible for EBB, with the exception of patients with a complete obstruction, which made an adequate positioning of the catheter impossible. These inclusion criteria did not change during the treatment period. Extrinsic tumours compressing the bronchus from outside were not considered appropriate for EBB. The third group (group XRTEBB) consisted of 62 patients $(6.6 \%$ ) who were primarily treated with a combination of EBB and XRT. The fourth group of patients (group EBBrec) consisted of 23 patients $(2.5 \%$ ) who were primarily treated with XRT and treated with EBB for a recurrence and finally the fifth group (group EBB) consisted of 13 patients $(1.4 \%)$ treated with EBB alone. This latter group mainly included patients with distant metastases and severe complaints due to endobronchial tumour needing treatment.

\section{Extemal beam radiotherapy}

All patients were treated with a linear accelerator with 8 to $16 \mathrm{MV}$ photons according to three different fractionation schemes, i.e. curative, radical or palliative.

Patients with distant metastases before radiotherapy, a poor general condition (WHO $\geq 2$ ) and/or advanced tumours (stage IIlb) were treated according to a palliative scheme. The target volume in this group encompassed the macroscopic tumour (primary tumour + 
enlarged lymph nodes) with a safety margin of $2 \mathrm{~cm}$, the ipsilateral hilus and the mediastinal lymph nodes on both sides. The supraclavicular fossa was only treated in case of involved lymph nodes. The field arrangements were assessed by direct simulation and correction for lung tissue density was not used. Fraction doses of 3 Gy were use up to a total dose of 30 Gy (4 times a week) by 2 opposing anterior-posterior/posterior-anterior fields.

A curative scheme was used in case of stage I or II tumours, which were smaller than 4 centimetres in patients with a WHO performance status of $\leq 2$. The initial target volume encompassed the primary tumour with a 2-centimetre margin, the ipsilateral hilus as well as the mediastinal lymph nodes on both sides. Before 1988 the supraclavicular lymph nodes were included electively on both sides. In patients, with very poor lung function and with peripherally located tumours without enlarged hilar lymph nodes, the initial target volume only included the primary tumour with a 2-centimetre margin. In this group a boost was given on the primary tumour with a 1-centimetre margin. The initial target volume was irradiated using fraction doses of 2.00 to $2.25 \mathrm{~Gy}$ to a total dose of 45 to $46 \mathrm{~Gy}$. The booster field was irradiated with fraction doses of $2.00 \mathrm{~Gy}$ to $2.50 \mathrm{~Gy}$ up to a cumulative dose of $70 \mathrm{~Gy}$. Field arrangements were assessed using CT-planning and corrections were made for lung tissue density. Individual lead blocking was used to shield normal tissue. In general, the initial target volume was treated with 2 opposing anterior-posterior/posterioranterior fields, while the booster was usually given by a three-field technique.

The remaining patients were treated according to the radical scheme. The initial target volume of the radical scheme encompassed the primary tumour with a 2-centimetre margin, the ipsilateral hilus as well as the mediastinal lymph nodes on both sides. Before 1988 the supraclavicular lymph nodes were included electively. The target volume of the booster field included the primary tumour and enlarged hilar lymph node with a 1-centimetre margin. The initial target volume was irradiated using fraction doses of 2.25 to $2.50 \mathrm{~Gy}$ to a total dose of $45 \mathrm{~Gy}$. The target of the booster field was irradiated with fraction doses of 2.50 Gy up to a cumulative dose of 60 Gy. Field arrangements were determined with a localisation/simulation procedure and optionally CT-assisted treatment planning was used. Corrections were made for lung tissue density. Individual lead blocking was used to shield normal tissues as much as possible. In general, the initial target volume was treated with 2 opposing anterior-posterior/posterior-anterior fields, while the booster was given by a different technique. The maximum dose to the spinal cord was equal or less than $45 \mathrm{~Gy}$. 
The doses mentioned were specified on the $100 \%$-isodose and the target volumes described were included in the $90 \%$-isodose.

In group URT, 55 patients (13\%) were treated according to the curative scheme, 268 patients $(64 \%)$ according to the radical scheme and 98 patients $(23 \%)$ according to the palliative scheme. In group XRTelig, 25 patient (6\%) was treated according to the curative scheme, 301 patients $(72 \%)$ according to the radical scheme and 93 patients $(22 \%)$ according to the palliative scheme. In group XRTEBB, 1 patient (2\%) was treated according to the curative scheme, $29(47 \%)$ according to the radical scheme and $32(52 \%)$ according to the palliative scheme.

\section{Endobronchial brachytherapy}

Patients who were treated before 1990 only received XRT. EBB was used from 1990 onwards, when an HDR-remote afterloading machine became available at our department. Initially, in all patients treated with EBB, a single dose of 15 Gy was used, calculated at a 1 centimetre distance of the central axis. In 1992, the dose was decreased to a single dose of $10 \mathrm{~Gy}$, based on the observation of a tendency towards a high incidence of massive haemoptysis in the group of patients treated with a single dose of $15 \mathrm{~Gy}$. In 1994, fractionation of EBB when combined with XRT was changed to 2 fractions of $7.5 \mathrm{~Gy}$, with reference to the reports of Speiser and co-workers ${ }^{22,23}$. EBB was administered at day 1 and day 8, while XRT started on day 4. XRT and EBB were not delivered at the same day. In case of a recurrence after XRT or in case of treatment with EBB alone, a single dose of 10 Gy was used. Under local anaesthesia the localisation and the extension of the endobronchial tumour were assessed by bronchoscopy. After bronchoscopic localisation, a plastic afterloading catheter was inserted beyond the endobronchial tumour and its position was verified by fluoroscopy and documented with orthogonal radiographs. The diameter of the catheter used was $2 \mathrm{~mm}$. The target volume consisted of the extent of the endobronchial tumour with a margin of 1 centimetre on both ends. When the distal border of the tumour was not visible by bronchoscopy, the distal extension was estimated using CTscan of the thorax. Then, the catheter was connected to the HDR-microselectron (Nucletron) and treatment was performed using an Iridium ${ }^{192}$ stepping source, using a stepping size of 2.5 or $5 \mathrm{~mm}$. All doses prescribed are specified at a 1 centimetre distance from the central axis of the source. In all patients a single catheter was used and dose 
optimisation was not performed. The median active length was $8 \mathrm{~cm}$, varying from 4 to 12 $\mathrm{cm}$.

In patients in group EBB, 3 out of 13 patients received a single fraction of $10 \mathrm{~Gy}, 9$ patients a single fraction of $15 \mathrm{~Gy}$ and one patient was treated with 2 fractions of $10 \mathrm{~Gy}$ with a 2 weeks interval at the tumour in the right main bronchus. This last patient received a single dose of 15 Gy 3 months later in the left main bronchus.

Two patients in group EBBrec received 2 fractions of $7.5 \mathrm{~Gy}, 10$ patients a single dose of 10 Gy and 11 patients a single dose of $15 \mathrm{~Gy}$. One patient was treated with a single dose of 10 Gy and treated again after 4 weeks at the same location with the same fraction dose because of persisting obstructive pneumonia. Four patients were re-treated with a single dose of $15 \mathrm{~Gy}$ and with a single fraction of $10 \mathrm{~Gy}$ for recurrent pulmonary symptoms due to endobronchial tumour some weeks or months later.

A total number of 26 patients in group XRTEBB received a single fraction of 15 Gy before starting XRT. Nine patients were treated with a single dose of $10 \mathrm{~Gy}$, while 27 patients were treated with 2 fractions of $7.5 \mathrm{~Gy}$.

After treatment the patients were observed and discharged after a few hours.

\section{Statistics}

A two-sided chi-square test was used to test differences concerning the frequencies of massive haemoptysis between groups. A logistic regression analysis was performed to identify independent prognostic factors related to the incidence of massive haemoptysis in a multivariate analysis. Results were regarded as statistically significant if the probability of chance occurrence was $5 \%$ or less.

Table 2: Treatment groups

\begin{tabular}{llcc}
\hline Group name & Treatment & Number & Percentage \\
\hline XRT & External beam radiotherapy (not eligible for brachytherapy) & 421 & $44.9 \%$ \\
XRTelig & External beam radiotherapy (eligible for brachytherapy) & 419 & $44.7 \%$ \\
XRTEBB & External beam radiotherapy + brachytherapy as primary treatment & 62 & $6.6 \%$ \\
EBBrec & Brachytherapy for recurrence after external beam radiotherapy & 23 & $2.5 \%$ \\
EBB & Brachytherapy alone as primary treatment & 13 & $1.4 \%$ \\
\hline
\end{tabular}




\section{Results}

Incidence of massive hemoptysis

From the 938 patients included in the study a total number of 101 patients $(10.8 \%)$ died from massive haemoptysis. Forty-eight patients $(5.1 \%)$ were alive at the time of the final analysis, meaning that $11.3 \%$ of the patients expired, died from massive haemoptyis. Seventy-three patients $(72 \%)$ suddenly died at home, the remaining patients expiring during admission in the hospital. In 78 cases (77\%), there was clinical or radiological evidence of local tumour progression, while in 23 patients there were no signs of local progression or local progression was not evaluable. The minimal follow up time was 12 months. The mean time from the first day of radiotherapy to massive haemoptysis was 10.5 months.

Incidence of massive haemoptysis by treatment-group

In group XRT, 18 out of 421 patients (4.3\%) died from massive haemoptysis (Table 2). This was significantly lower $(p<0.001)$ as compared to group XRTelig, in which 55 out of 419 patients (13.1\%) died from massive haemoptysis. In group XRTEBB, 16 out of 62 patients (25.8\%) died from massive haemoptysis, which was significantly higher as compared to patients in group XRT as well as in group XRTelig $(p=0.009)$. Of the 23 patients in group EBBrec, 10 patients (43.4\%) died of massive haemoptysis, while in group EBB, 2 out of 13 patients $(15.4 \%)$ died from massive haemoptysis.

\section{Incidence of massive haemoptysis in group EBBrec}

In the group of 10 patients treated for recurrence with a total dose of EBB which was 10 Gy or less, 4 cases (40\%) died of massive haemoptysis. After a total dose of $15 \mathrm{~Gy}, 2$ out of 9 patients (22\%) died of massive haemoptysis and in the 4 patients treated with repeated EBB up to a total dose ranging form 20 to $35 \mathrm{~Gy}$ for persistent tumour with respiratory symptoms, all 4 expired by massive haemoptysis.

Four out of 23 patients treated for relapse with EBB were previously irradiated according to the curative scheme, 10 according to the radical scheme and 9 according to the palliative scheme. The number of patients expiring from massive haemoptysis was 2 after curative radiotherapy $(50 \%), 5$ after radical radiotherapy $(50 \%)$ and 3 after palliative radiotherapy (33\%). 
Table 3: Incidence of massive haemoptysis in group XRTelig and XRTEBB

\begin{tabular}{|c|c|c|c|c|c|}
\hline Variable & & $\begin{array}{c}\text { Total } \\
\text { number }\end{array}$ & $\begin{array}{c}\text { Number } \\
\text { massive } \\
\text { haemoptysis }\end{array}$ & $\begin{array}{c}\text { Percentage } \\
\text { massive } \\
\text { haemoptysis }\end{array}$ & $\begin{array}{l}\text { P-value chi- } \\
\text { square } \\
\text { (2-sided) }\end{array}$ \\
\hline \multirow[t]{2}{*}{ Sex } & Female & 52 & 7 & $13.5 \%$ & $p=0.78$ \\
\hline & Male & 429 & 64 & $14.9 \%$ & \\
\hline \multirow[t]{2}{*}{ Age } & $0-70$ years & 296 & 45 & $15.2 \%$ & $p=0.73$ \\
\hline & $>70$ years & 185 & 26 & $14.1 \%$ & \\
\hline \multirow[t]{5}{*}{ T-classification } & T1 & 14 & 0 & $0 \%$ & $p=0.28$ \\
\hline & $\mathrm{T} 2$ & 207 & 30 & $14.5 \%$ & \\
\hline & T3 & 109 & 20 & $18.3 \%$ & \\
\hline & T4 & 150 & 20 & $13.3 \%$ & \\
\hline & unknown & 1 & 1 & & \\
\hline \multirow[t]{3}{*}{$\mathrm{N}$-classification } & No & 153 & 31 & $20.3 \%$ & $p=0.026$ \\
\hline & $\mathrm{N}+$ & 299 & 37 & $12.4 \%$ & \\
\hline & unknown & 29 & 3 & & \\
\hline \multirow{3}{*}{$\begin{array}{l}\text { Haemoptysis before } \\
\text { radiotherapy }\end{array}$} & Yes & 158 & 30 & $19.0 \%$ & $p=0.07$ \\
\hline & No & 321 & 41 & $12.8 \%$ & \\
\hline & unknown & 2 & 0 & & \\
\hline \multirow[t]{2}{*}{ Localisation } & Upper lobes & 244 & 46 & $18.9 \%$ & $p=0.011$ \\
\hline & Other localisations & 237 & 25 & $10.5 \%$ & \\
\hline \multirow[t]{4}{*}{ Histology } & Squamous cell & 342 & 59 & $17.3 \%$ & $p=0.016$ \\
\hline & Adenocarcinoma & 56 & 2 & $3.6 \%$ & \\
\hline & Large cell carcinoma & 80 & 9 & $11.3 \%$ & \\
\hline & Others & 3 & 1 & & \\
\hline \multirow[t]{2}{*}{ Site } & Right & 266 & 33 & $12.4 \%$ & $p=0.12$ \\
\hline & Left & 215 & 38 & $17.8 \%$ & \\
\hline Intrabronchial tumour & Lobar bronchus & 153 & 11 & $7.2 \%$ & $p=0.001$ \\
\hline extension & Main bonchus T2 & 173 & 36 & $20.8 \%$ & \\
\hline \multirow[t]{2}{*}{ (Lobar versus others) } & Main bronchus T3 & 119 & 18 & $15.1 \%$ & \\
\hline & Trachea & 35 & 6 & $17.1 \%$ & \\
\hline \multirow[t]{4}{*}{ Brachytherapy } & No brachytherapy & 419 & 55 & $13.1 \%$ & $p<0.001$ \\
\hline & 7.5 Gray per fraction & 27 & 3 & $11.1 \%$ & \\
\hline & 10.0 Gray per fraction & 9 & 1 & $11.1 \%$ & \\
\hline & 15.0 Gray per fraction & 26 & 12 & $46.2 \%$ & \\
\hline \multirow[t]{2}{*}{ External radiotherapy } & Low dose (0-45 Gy) & 125 & 20 & $16.0 \%$ & $p=0.65$ \\
\hline & High dose (46-70 Gy) & 356 & 51 & $14.3 \%$ & \\
\hline
\end{tabular}




\section{Univariate analysis of FFMH in group EBBelig and XRTEBB}

The patients out of group XRTelig and XRTEBB were further analysed to identify risk factors for massive haemoptysis in patients eligible for brachytherapy irrespective of the admission of brachytherapy. In the univariate analysis, in turned out that a higher incidence of massive haemoptysis was observed in case of clinically NO-disease, squamous cell carcinoma, intrabronchial tumour extension into the main bronchus, localisation in the upper lobes and a fraction size used for EBB of 15 Gy (Table 3). The incidence of massive haemoptysis in patients primarily treated with 2 fraction of $7.5 \mathrm{~Gy}$ brachytherapy or 1 fraction of $10 \mathrm{~Gy}$ brachytherapy followed by XRT was $11.1 \%$ in both groups and did not differ from patients in group XRTelig.

In the groups of patients XRTelig and XRTEBB, 26 patients were treated according to the curative scheme and 1 of these patients (4\%) was also treated EBB ( $1 \times 15 \mathrm{~Gy})$. In the group of 318 patients irradiated according to the radical scheme, 29 patients $(9 \%)$ were treated with EBB as well. Twenty patients (6\%) in this group received 7.5 Gy fractions, 2 patients (1\%) 10 Gy fractions and 7 patients (2\%) 15 Gy fractions. In the group of 125 patients treated according to the palliative scheme, 32 cases $(26 \%)$ also received EBB of which 7 patients $(6 \%)$ received 7.5 Gy fractions, 7 patients $(6 \%) 10$ Gy fractions and 18 patients (14\%) 15 Gy fractions.

To test the hypothesis whether a 15 Gy single dose was tolerable after palliative radiotherapy and not after radical or curative radiotherapy, the group of patients was divided in a low dose XRT group ( $30 \mathrm{~Gy}$ ) and a high dose XRT group (60 or $70 \mathrm{GY}$ ). In the low dose XRT group, 1 out of 13 patients (8\%) treated with a fraction dose of 7.5 or $10 \mathrm{~Gy}$, and 9 out of 18 patients $(50 \%)$ treated with a fraction dose of $15 \mathrm{~Gy}$, died of massive haemoptysis. In the high dose XRT group, 3 out of 22 patients (14\%) treated with a fraction dose of 7.5 or 10 $\mathrm{Gy}$, and 3 out of 8 patients (38\%) treated with a fraction dose of $10 \mathrm{~Gy}$, died of massive haemoptysis. Although the numbers are rather small, there is no indication that a single dose of 15 Gy was less tolerable after high dose XRT.

Multivariate analysis of the incidence of massive haemoptysis in group EBBelig and XRTEBB

Intrabronchial tumour extension in the main bronchus, haemoptysis before radiotherapy and localisation of the tumour in the upper bronchus turned out to be independently associated 
Table 4: results of the multivariate analysis with regard to the FFMH. Only the variables significantly associated with FFMH are shown

\begin{tabular}{lccccc}
\hline Variable & Wald & df & p-value & RR & (95\%-CI) \\
\hline Fraction size EBB: $1 \times 15$ Gy at $1 \mathrm{~cm}$ & 12.0 & 1 & $\mathrm{p}<0.001$ & 5.3 & $(2.1-13.7)$ \\
Localisation upper lobe & 10.3 & 1 & $\mathrm{p}<0.005$ & 2.7 & $(1.5-5.1)$ \\
Intrabronchial tumour extension into main bronch 656 & 1 & $\mathrm{p}<0.01$ & 2.7 & $(1.3-5.9)$ \\
Haemoptysis before radiotherapy & 6.3 & 1 & $\mathrm{p}<0.05$ & 2.1 & $(1.2-3.9)$ \\
\hline
\end{tabular}

Note: The same multivariate analysis was performed in group XRTlig and XRTEBB after the patients treated with a fraction dose of $15 \mathrm{~Gy}$ had been excluded. In this second multivariate analysis, a fraction dose of 7.5 t $10 \mathrm{~Gy}$, was not associated with a higher incidence of massive haemoptysis.

with the incidence of massive haemoptysis (Table 4). The most striking finding was a clear dose-response relationship between fraction dose of EBB and massive haemoptysis. An additional analysis was performed on the incidence of massive haemoptysis and variables significantly related to the FFMH after stratification for fraction dose of brachytherapy. In all subsets, higher incidence rates of massive haemoptyis were noted after a fraction dose of $15 \mathrm{~Gy}$, particularly in tumours located in the upper lobes (Table 5).

\section{Discussion}

First, the incidence of massive haemoptysis after XRT alone (group XRT and XRTelig) was $8.7 \%$. This incidence rate is comparable with the results of other investigators, reporting on the incidence of massive haemoptysis in NSCLC treated with XRT alone. Miller and McGregor ${ }^{16}$ reported on the incidence of massive haemorrhage in 877 lung cancer patients treated with different modalities. Twenty-nine patients $(3.3 \%)$ died of massive haemorrhage. In the group of patients treated with XRT, 13 out of 116 patients (11.2\%) died of massive haemoptysis. However, radiotherapy was not statistically significant associated with massive haemoptysis. Recently, Huber and co-workers ${ }^{11}$ presented a prospective randomised trial in which patients with squamous cell carcinoma were randomised between XRT alone and XRT with additional EBB. In the group of 42 patients treated with XRT alone, 6 patients (14.3\%) died from massive haemoptysis. The results of these reports as well as the results of the present study point out, that in patients with 
Table 5: Incidence of massive haemoptysis stratified by fraction size of EBB in variables significantly associated with FFMH.

\begin{tabular}{|c|c|c|c|c|c|}
\hline Variable & & EBB & $\begin{array}{c}\text { Number } \\
\text { massive } \\
\text { haemoptysis }\end{array}$ & $\begin{array}{c}\text { Percentage } \\
\text { massive } \\
\text { haemoptysis }\end{array}$ & $\begin{array}{l}\text { P-value chi- } \\
\text { square } \\
\text { (2-sided) }\end{array}$ \\
\hline \multirow[t]{6}{*}{ Localisation } & Upper lobes & no EBB & 222 & 37 & $16.7 \%$ \\
\hline & & $1 \times 10$ Gy or $2 \times 7.5$ Gy & 13 & 2 & $15.4 \%$ \\
\hline & & $1 \times 15 \mathrm{~Gy}$ & 9 & 7 & $77.8 \%$ \\
\hline & Other lobes & no EBB & 197 & 18 & $9.1 \%$ \\
\hline & & $1 \times 10$ Gy or $2 \times 7.5 \mathrm{~Gy}$ & 23 & 2 & $8.7 \%$ \\
\hline & & $1 \times 15 \mathrm{~Gy}$ & 17 & 5 & $29.4 \%$ \\
\hline Haemoptysis & Yes & no $E B B$ & 139 & 26 & $18.7 \%$ \\
\hline \multirow[t]{5}{*}{ before therapy } & & $1 \times 10$ Gy or $2 \times 7.5$ Gy & 12 & 2 & $16.7 \%$ \\
\hline & & $1 \times 15 \mathrm{~Gy}$ & 5 & 2 & $40.0 \%$ \\
\hline & No & no EBB & 278 & 29 & $10.4 \%$ \\
\hline & & $1 \times 10 \mathrm{~Gy}$ or $2 \times 7.5 \mathrm{~Gy}$ & 23 & 2 & $8.7 \%$ \\
\hline & & $1 \times 15$ Gy & 20 & 10 & $50.0 \%$ \\
\hline Intrabronchial tumour & Yes & no EBB & 272 & 45 & $16.5 \%$ \\
\hline \multirow[t]{5}{*}{ in main bronchus } & & $1 \times 10$ Gy or $2 \times 7.5$ Gy & 29 & 3 & $10.3 \%$ \\
\hline & & $1 \times 15 \mathrm{~Gy}$ & 26 & 12 & $46.2 \%$ \\
\hline & No & no EBB & 146 & 10 & $6.8 \%$ \\
\hline & & $1 \times 10$ Gy or $2 \times 7.5$ Gy & 7 & 1 & $14.3 \%$ \\
\hline & & $1 \times 15$ Gy & 0 & & \\
\hline
\end{tabular}

inoperable NSCLC treated with XRT alone, the probability of expiring as a result of massive haemoptysis is about $10 \%$.

Secondly, we observed a statistically significant higher incidence of massive haemoptysis in patients treated with XRT alone but eligible for EBB as compared to patients treated with XRT and not eligible for EBB. In the report of Miller and McGregor ${ }^{16}$, massive haemoptysis turned out to be significantly associated with cavitated squamous cell carcinoma arising in the right or left main bronchus. In general, these patients are suitable candidates for EBB. These findings strongly suggest that the high incidence of massive haemoptysis described in reports concerning treatment with EBB is at least partly due to selection bias. The higher incidence of massive haemoptysis in this subset of patients could be explained by the fact that these tumours were centrally located and lying in the near vicinity of the major vessels. The right pulmonary artery directly lies over the anterior surface of the right main bronchus and right upper lobe, while the left pulmonary artery lies directly over the upper surface of the left main bronchus and left upper lobe. Pearlberg and co- 
workers ${ }^{18}$ performed radiological investigations of tumour vicinities prior to laser therapy and found that the major vessels were located just 1 to $3 \mathrm{~mm}$ away from bronchial tumours in the left or right main bronchus in four of ten patients. This explanation is also supported by the results of the multivariate analysis of the present study, in which endobronchial tumour extension into the main bronchus and the presence of haemoptysis before radiotherapy were independent risk factors for massive haemoptysis. From this point of view, the presence of haemoptysis before radiotherapy could be regarded as a predictive factor for tumour extension into one of the major vessels.

The development of a bronchovascular fistula can occur by three different mechanisms. Firstly, when the tumour already extends through the wall of the vessel before radiotherapy, rapid regression or necrosis of the tumour due to irradiation may lead to the development of a bronchovascular fistula. Secondly, a bronchovascular fistula could be due to direct tumour progression, which was the case in the majority of patients dying from massive haemoptysis in the present study. Finally, a fistula may result of necrosis of the bronchial mucosa and vessel wall by radiotherapy itself. We did not find a correlation between the total dose of XRT and massive haemoptysis. However, in many patients who died from massive haemoptysis it remains unclear if radiotherapy itself contributed to the development of a bronchovascular fistula or was due to other causes. As shown in this study, the majority of patients died at home and general practitioners were often reluctant to discuss the issue of autopsy with the family just after such a striking event. Furthermore, even when residual or recurrent tumour is found at autopsy, it does not rule out XRT as contributing factor.

One of the most important issues of the present study was whether the addition of EBB to $X R T$ contributed to a higher incidence of massive haemoptysis. To investigate this, patients in group XRTelig and XRTEBB were analysed separately from the other treatment groups, as these patients were all eligible for EBB, irrespective of the actual treatment performed. Initially, the incidence of massive haemoptysis was significantly higher in group XRTEBB as compared to XRTelig. However, the most striking finding was the significant association of the fraction size of $E B B$ and the incidence of massive haemoptysis. After a combination of a single dose of 15 Gy with EBB in addition to XRT, $46.2 \%$ of the patients died of massive haemoptysis, which was significantly higher as compared to after a single dose of 10 Gy or 2 fractions of 7.5 Gy EBB in addition to XRT. With the latter 2 fractionations of EBB the incidence of massive haemoptysis did not differ 
significantly from the incidence observed in patients in group XRTelig. The incidence of massive haemoptysis was especially high when a fraction dose of $15 \mathrm{~Gy}$ was used in tumours of the upper lobes. As we specified the dose on 1 centimetre of the central axis of the source and the diameter of the catheter used was $3 \mathrm{~mm}$, the actual dose at the surface of the catheter is much higher. Assuming a linear fall in dose in the case of a line source, the dose at the surface of the catheter is 6 to 7 times higher as compared to the prescribed dose at $1 \mathrm{~cm}$. When adjustments are made for the biological effective dose, the dose at the surface of the catheter is even higher for early responding tissues as well as for late responding tissues. Especially, in case of tumours located in one of the upper lobes, the catheter lies immediately adjacent to the mucosa of the bronchial wall. Therefore, it is conceivable that such high doses might contribute to the development of mucosal necrosis and bronchovascular fistula. Furthermore, a number of series reporting on EBB in lung cancer showed that a few patients who died from massive haemoptysis did not show any evidence of tumour at autopsy, suggesting that EBB contributed at least partly to the development of massive haemoptysis ${ }^{3,4}$. In most studies reporting on the results and complications of primary treatment of EBB and XRT, fraction doses used for HDR-brachytherapy are ranging from 4 to $10 \mathrm{~Gy}$ per fraction and incidence rates for massive haemoptysis are found ranging from $0 \%$ to $20.8 \%{ }^{1,3,5,7,11,17,19,23,25}$. The incidence of massive haemoptysis found in our series in the group of patients treated with fraction doses of $7.5 \mathrm{~Gy}$ or $10 \mathrm{~Gy}$ in combination with XRT are comparable with the results reported in these studies. In a retrospective analysis, in which differences between historically based treatment approaches are compared, it can not ruled out that the high incidence in the group treated with 15 Gy brachytherapy and external irradiation is partly due to selection bias. However, in spite of this possible bias effect, the difference found in this study is so large, that it can not be ignored and explained by selection bias alone. Moreover, a dose-response relationship was also noted in the series described by Cotter and co-workers ${ }^{5}$. They added the total dose of XRT and EBB. When a total dose of $\geq 85$ Gy was used, the incidence of severe complications increased to $33 \%$ in the HDR-group, as compared to $11 \%$ when the total dose ranged from 70 to 85 Gy while no severe complication were noted when the total dose was $<70 \mathrm{~Gy}$.

The incidence of massive haemoptysis after EBB alone was $15.4 \%$. Gollins and coworkers ${ }^{8}$ reported on the results of EBB in 406 patients with carcinoma of the bronchus, 
the majority treated by EBB as single treatment modality. In a multivariate analysis with regard to risk factors for massive haemoptysis, they found a dose-response relationship towards the risk of massive haemoptysis when the dose per fraction was increased from $15 \mathrm{~Gy}$ to $20 \mathrm{~Gy}$. Other risk factors identified in these series were prior laser treatment at the site of EBB and second EBB in the same location as the first EBB. The incidence of massive haemoptysis after EBB 15 Gy was only 6\%. When EBB 15 Gy was used in 17 patients with XRT (usually 30 Gy in 8 fractions) the incidence was $18 \%$ and caution was advised when EBB and XRT are given concurrently.

The incidence of massive haemoptysis in group EBB was comparable with the incidence in group XRTEBB, also pointing out that the contribution of XRT to the development of a bronchovascular fistula is probably limited.

The incidence of massive haemoptyis in the group of patients treated with brachytherapy for a recurrence was $43.4 \%$. A number of studies concerning brachytherapy for recurrence after external irradiation reported on the incidence of this fatal complication ranging from 0 to $50 \%^{2,6,9,10,12,13,14,15,17,19,20,21,24}$. The rate of massive haemoptysis in this treatment group was significantly higher as compared to the other treatment groups. Possibly, brachytherapy prevents bronchial obstruction and thus respiratory insufficiency, while the extrabronchial tumour more distantly from the catheter, is inadequately treated because of the rapid dose fall off. However, the incidence rate seems to be somewhat higher as compared to the rates reported in the literature. Considering the relatively high fraction doses used in this treatment group, EBB can not be ruled out as contributing factor in this subset as patients as well.

On the basis of the results of this retrospective study, we conclude that the relatively high incidence of massive haemoptysis after endobronchial brachytherapy described in the literature is at least partly due to selection bias of patients who are eligible for EBB. In the interpretation of studies mentioning rates of massive haemoptysis after EBB, an incidence rate of about $10 \%$ after XRT alone has to be kept in mind.

Secondly, the results of this study strongly suggest that a fraction dose of 15 Gy at a 1 centimetre distance from the source axis lead to an unacceptable risk of massive haemoptysis, in particular when EBB is added to XRT. Therefore, we strongly recommend not using such a high dose per fraction in endobronchial brachytherapy, especially if brachytherapy is combined with external irradiation in the primary treatment of inoperable 
NSCLC. Nowadays, we use two fractions of $7.5 \mathrm{~Gy}$ when EBB is combined with XRT and a single dose of $10 \mathrm{~Gy}$ for recurrence after XRT. 


\section{References}

1. Aygun C, Weiner S, Scariato A, Spearman D and Stark L. Treatment of non-small cell lung cancer with external beam radiotherapy and high dose rate brachytherapy. Int J Radiat Oncol Biol Phys 1992; 23: $127-132$.

2. Bedwinek J, Petty A, Bruton $C$, Sofield $J$ and Lee $L$. The use of high dose rate endobronchial brachytherapy to palliate symptomatic endobronchial recurrence of previously irradiated bronchogenic carcinoma. Int J Radiat Oncol Biol Phys $1991 ; 22: 23-30$.

3. Chang LL, Horvath J, Peyton $\mathrm{W}$, and Ling $\mathrm{S}$. High dose rate afterloading intraluminal brachytherapy in malignant airway obstruction of lung cancer. Int J Radiat Oncol Biol Phys 1994; 28 : 589-596.

4. Cotter GW, Herbert DE and Ellingwood KE. Inoperable endobronchial obstructing lung carcinoma treated with combined endobronchial and external beam irradiation. Southern Medical Journal 1991; 84 (5): 562-565.

5. Cotter GW, Lariscy C, Ellingwood KE and Herbert D. Inoperable endobronchial obstructing lung carcinoma treated with combined endobronchial and external beam irradiation: a dosimetric analysis. Int J Radiat Oncol Biol Phys 1993; 27: 531-535.

6. Gauwitz $M$, Ellerbroek N, Komaki R et al. High dose endobronchial irradiation in recurrent bronchogenic carcinoma. Int J Radiat Oncol Biol Phys 1992; 23: 397-400.

7. Goldman JM, Bulman AS, Rathmell AJ, et al. Physiological effect of endobronchial radiotherapy in patients with major airway occlusion by carcinoma. Thorax 1993; 48: 110-114.

8. Gollins SW, Ryder WDJ, Burt PA, et al. Massive haemoptysis death and other morbidity associated with high dose rate radiotherapy for carcinoma of the bronchus. Radiother Oncol 1996; 39: 105-116.

9. Hernandez P, Gursahaney A, Roman T, et al. High dose rate brachytherapy for the local control of endobronchial carcinoma following external irradiation. Thorax 1996; 51: 354-358.

10. Huber RM, Fischer $R$, Hautmann $H$, et al. Palliative endobronchial brachytherapy for central lung tumors. $A$ prospective, randomised comparison of two fractionation schedules. Chest 1995; 107 (2): 463-470.

11. Huber RM, Fischer $\mathrm{R}$, Hautmann $\mathrm{H}$, et al. Does additional brachytherapy improve the effect of external irradiation? A prospective, randomised study in central lung tumours. Int J Radiat Oncol Biol Phys 1997; 38 (3): 533-540.

12. Khanavkar B, Stern $P, A l b e r t i W$ and Nakhosteen JA. Complications associated with brachytherapy alone or with laser in lung cancer. Chest 1991; 99: 1062-1065.

13. Lo TCM, Girshovich L, Healey GA, et al. Low dose rate versus high dose rate intraluminal brachytherapy for malignant endobronchial tumours. . Radiother Oncol 1995; 35: 193-197.

14. Mehta MP, Shahabi S, Jarjour NN and Kinsella TJ. Endobronchial irradiation for malignant airway obstruction. Int $J$ Radiat Oncol Biol Phys 1989; 17: 847-851.

15. Micke $O$, Prott FJ, Schäfer U, et al. Endoluminal HDR brachytherapy as a palliative treatment of patients with recurrent previously irradiated non-small cell lung carcinoma. Strahlenther Onkol 1995; 171: 554-559.

16. Miller RR and McGregor DH. Haemorrhage from carcinoma of the lung. Cancer 1980; 46: 200-205.

17. Nori D, Allison R, Kaplan B, et al. High dose rate intraluminal irradiation in bronchogenic carcinoma. Chest 1993; 104: 1006-1011.

18. Pearlberg JL, Sandler MA, Kvale $P$, ef al. Computed-tomographic and conventional linear tomographic evaluation of tracheobronchial lesions for laser photoresection. Radiology $1985 ; 154: 759-762$.

19. Schraube P, Fritz P, Becker HD and Wannenmacher M. Results of Endoluminal high-dose-rate irradiation of central 
non-small cell lung cancer. Strahlenther Onkol 1993; 169: 228-234.

20. Schray M, MCDougall JC, Martinez $A$, et al. Management of malignant airway compromise with laser and low dose rate brachytherapy. Chest 1988; 93: 264-269.

21. Seagren SL, Harrell JH and Hom RA. High dose rate intraluminal irradiation in recurrent endobronchial carcinoma. Chest 1985; 88 (6): 810-14.

22. Speiser BL and Sprating $\mathrm{L}$. Remote afterloading brachytherapy for the local control of endobronchial carcinoma. Int $\mathrm{J}$ Radiat Oncol Biol Phys 1993; 25: 579-587.

23. Speiser BL and Spratling $\mathrm{L}$. Radiation bronchitis and stenosis secondary to high dose rate endobronchial irradiation. Int J Radiat Oncol Biol Phys 1993; 25: 589-597.

24. Sutedja G, Baris G, Schaake-Koning $C$ and van Zandwijk N. High dose rate brachytherapy in patients with local recurrences after radiotherapy of non-small cell lung cancer. Int J Radiat Oncol Biol Phys 1992; 24: 551-553.

25. Zajac AJ, Kohn ML, Heiser D and Peters JW High-dose-rate intraluminal brachytherapy in the treatment of endobronchial malignancy. Radiology 1993; 187: 571-575. 
Chapter 10

\title{
External irradiation versus external irradiation plus endobronchial brachytherapy in inoperable non-small cell lung cancer: A prospective randomised study.
}

\author{
Hans Langendijk \\ Jos de Jong \\ Martin Tjwa \\ Martin Muller \\ Guul ten Velde \\ Neil Aaronson \\ Ben Slotman \\ Miel Wouters
}




\section{Abstract}

Purpose: No randomized studies are available on the additional value of EBB to XRT regarding palliation of respiratory symptoms (RS). A prospective randomized study was initiated to test the hypothesis that the addition of EBB to XRT provides higher levels of palliation of RS and improvement of quality of life (QoL) in patients with NSCLC with endobronchial tumor.

Material and methods: Patients with previously untreated NSCLC, stages I-IIlb, WHOperformance status of 0-3 and with histologic proven endobronchial tumor in the proximal airways were eligible. EBB consisted of two fractions of $7.5 \mathrm{~Gy}$ at $1 \mathrm{~cm}$ on day 1 and 8 . XRT started at day 2. The XRT dose was $30 \mathrm{~Gy}$ (2 weeks) or 60 Gy (6 weeks). The EORTC QLQ-C30 and QLQ-LC13 were assessed before treatment and 2 weeks, 6 weeks, 3, 6 and 12 months after treatment. Re-expansion of collapsed lung was tested by the inspiratory vital capacity (IVC) and CT scan of the chest.

Results: 95 patients were randomized between arm 1 (XRT alone)( $n=48)$ or arm 2 (XRT + $\mathrm{EBB})(n=47)$. The arms were well balanced regarding pre-treatment characteristics and QoL scores. The compliance for QoL-assessment was $>90 \%$ at all times. A beneficial effect of EBB was noted concerning palliation of dyspnea over time $(p=0.02)$, which lasted for 3 months. This benefit was only observed among patients with an obstructing tumor of the main bronchus. No differences between the 2 arms were noted with regard to other RS and QoL.

A higher rate of re-expansion of collapsed lung was observed in arm $2(57 \%)$ compared to arm $1(35 \%)(p=0.01)$. The IVC assessed 2 weeks after radiotherapy improved with $493 \mathrm{cc}$ in arm 2 and decreased $50 \mathrm{cc}$ in arm $1(\mathrm{p}=0.03)$.

No difference was noted regarding the incidence of massive haemoptysis (13\% versus $15 \%)$.

Conclusion: The addition of EBB to XRT in NSCLC is save and provides higher rates of re-expansion of collapsed lung resulting in better palliation of dyspnea. $A$ beneficial effect of EBB was also noted for haemoptysis. 


\section{Introduction}

Radiotherapy is an effective treatment modality in the palliation of most respiratory symptoms among patients with inoperable non-small cell lung cancer (NSCLC) ${ }^{1-4}$. In two randomized studies conducted by the Medical Research Council (MRC), different palliative schedules were compared and the rates of symptom relief varied from $48 \%$ to $65 \%$ for cough, from $72 \%$ to $86 \%$ for haemoptysis and from $59 \%$ to $80 \%$ for chest pain ${ }^{1-2}$.

In a large number of studies, the results of endobronchial brachytherapy (EBB) with or without external irradiation (XRT) as palliative treatment for centrally localized lung cancer have been reported ${ }^{5-11}$. In earlier studies, EBB was merely used as palliative treatment in case of endobronchial recurrences after XRT. In this category of patients, EBB offered adequate symptomatic relief in a substantial proportion of patients ${ }^{10,12-18}$. Later, EBB was also combined with XRT as primary treatment ${ }^{6,11,19,20}$. Speiser and co-workers ${ }^{11}$ reported on a prospective study among 342 patients with endobronchial tumors treated by the combination of XRT ( 30 to $60 \mathrm{~Gy}$ ) and concomitant EBB during week 1, 3 and 5 . The results achieved with this approach were quite remarkable, with response rates of $99 \%$ for haemoptysis, $85 \%$ for cough and $86 \%$ for dyspnea. In a retrospective study, Chang and co-workers ${ }^{6}$ reported comparable results with the combination of XRT (20 to $70 \mathrm{~Gy}$ ) and concomitant EBB ( $3 \times 7$ Gy HDR) during week 2,4 and 6 . They observed response rates of $79 \%$ for cough, $95 \%$ for haemoptysis and $87 \%$ for dyspnea.

Many patients with centrally localized lung cancer present with post-obstructive pneumonia or atelectasis. With XRT alone, the proportion of patients in whom a partial or complete re-expansion of collapsed lung has been achieved varies from $21 \%$ to $61 \%{ }^{21-23}$. With the combination of EBB and XRT, much higher rates of re-expansion have been observed, varying from $67 \%$ to $99 \%{ }^{6,11,19}$.

These results suggest that with the combination of XRT and EBB, post-obstructive features such as pneumonitis and atelectasis are likely to be treated more adequately compared to XRT alone. This could also account for the higher response rates for those respiratory symptoms associated with these post-obstructive features, in particular, dyspnea.

However, the definitions used for response of symptoms, re-expansion of collapsed lung, and the methods used to assess these responses, differed widely between the 
aforementioned studies. Moreover, the inclusion criteria of these studies differed widely and no randomized studies have been published investigating the additional value on palliation of respiratory symptoms of EBB plus XRT versus XRT alone. Therefore, the additional value of EBB concomitantly with XRT is not yet well defined.

This prospective randomized phase III study was conducted to test two specific hypotheses. Firstly, we expected that among patients with centrally localized NSCLC, the addition of concomitant EBB during XRT would result in higher rates of re-expansion of post-obstructive infiltration/atelectasis and/or higher rates of prevention of these postobstructive features. Secondly, we expected that these higher rates of re-expansion would result in higher levels of palliation of respiratory symptoms, in particular for dyspnea and cough, as well as in greater levels of improvement in quality of life (QoL). Furthermore, the higher dose that can be administered with EBB to the endobronchial-obstructing tumor may lead to higher a degree of desobstruction and an additional beneficial effect on respiratory symptoms, even in the absence of an atelectasis.

\section{Material and methods}

\section{Eligibility criteria}

To be eligible for the study, patients had to have histologically proven NSCLC, stage I, II, Illa or IIIb disease according to the UICC $1992{ }^{24}$, endobronchial tumor in the proximal main bronchus or lobar bronchus, a World Health Organisation performance status 0-3 and no prior or planned chemotherapy, prior surgery, prior radiotherapy, other malignancies, pleuritis carcinomatosa, distant metastases or superior vena cava syndrome. Prior treatment with Neodymium-YAG laser was not allowed and, therefore, patients with a complete obstruction were not eligible. The local ethical committees of the participating centers approved the study and all patients provided written informed consent.

\section{Study design}

Eligible patients were randomized to receive external irradiation alone (treatment arm 1) or external irradiation in combination with endobronchial brachytherapy (treatment arm 2). For 
external irradiation, two fractionation schedules were allowed, i.e. a radical fractionation schedule $(60 \mathrm{~Gy})$ or a palliative fractionation schedule (30 Gy). For both fractionation schedules, the target volume included the primary tumor and enlarged mediastinal and supraclavicular lymph nodes with a margin of two centimeters, as well as the mediastinal lymph node areas on both sides. For patients treated according to the palliative schedule, the target volume was irradiated with 3 Gy per fraction (4 times a week) up to a total dose of $30 \mathrm{~Gy}(100 \%)$ without correction for lung tissue density. For patients treated according to the radical schedule, the aforementioned target volume was treated with fraction doses of $2.25 \mathrm{~Gy}$ ( 4 times a week) to a total dose of $45 \mathrm{~Gy}$, followed by a boost up to $60 \mathrm{~Gy}$, using fraction doses of $2.5 \mathrm{~Gy}$ (4 times a week). When the radical schedule was used, correction was made for lung tissue density (0.3). In case of the presence of atelectasis, resulting in difficulties in distinguishing tumor from collapsed lung, all abnormalities were included in the target volume. Patients allocated to treatment arm 2 received an additional two fractions of 7.5 Gy with high dose rate (HDR) EBB in week 1 and 2. The choice of the external fractionation schedule was left to the decision of the treating physician. Stratification was applied for the external fractionation schedule (palliative; radical), stage (stage I, II; stage IIla, IIIb) and the participating center (Radiotherapeutisch Instituut Limburg, Heerlen and Maastricht; Verbeeten Instituut, Tilburg; The Antoni van Leeuwenhoek Hospital, Amsterdam).

\section{Endobronchial brachytherapy}

Under local anesthesia, the localization and the extension of the endobronchial tumor were assessed by bronchoscopy. After bronchoscopic localization, a plastic afterloading catheter was inserted beyond the endobronchial tumor and its position was verified by fluoroscopy and documented with orthogonal radiographs. The diameter of the catheter used was $3 \mathrm{~mm}$. The target volume consisted of the extent of the endobronchial tumor with a margin of one centimeter on both ends. When the distal border of the tumor was not visible by bronchoscopy, the distal extension was estimated using CT scan of the thorax. Then, the catheter was connected to the HDR-microselectron (Nucletron, Leersum, The Netherlands) and treatment was performed using an Iridium ${ }^{192}$ stepping source, using a stepping size of 2.5 or $5 \mathrm{~mm}$. All doses used were prescribed on a distance of one centimeter perpendicularly to the central axis of the source. In all patients, a single catheter was used 
and dose optimization was not performed. EBB and XRT were not administered on the same day. Following treatment, the patients were observed and discharged after a few hours.

\section{Staging procedures and follow up assessments}

The staging procedure consisted of a physical examination, postero-anterior and lateral chest radiography, CT scan of the chest including the liver and adrenal glands, and bronchoscopy in all patients. Lung function tests were performed by spirometry. Bone scintigraphy was performed only when indicated.

In the fourth week during radiotherapy, a chest radiograph was made to evaluate whether it was necessary to change the original field set up for the boost, because of changes due to re-expansion of atelectasis or post-obstructive pneumonia.

Definitive re-expansion of atelectasis or post-obstructive pneumonia was assessed by means of chest radiographs, CT scan of the chest and by changes in the inspiratory vital capacity (IVC), performed 2 to 6 weeks after the end of the entire course of treatment.

\section{Quality of life assessment}

To assess changes in quality of life (QoL) and respiratory symptoms, the Dutch version of the EORTC QLQ-C30 (version 1.0) and the lung cancer module QLQ-LC13 were used ${ }^{24-25}$. The EORTC QLQ-C30 is composed of 30 questions organized into five functional scales (physical, role, cognitive, emotional and social), three symptom scales (fatigue, pain and nausea and vomiting), a global health/quality of life scale, and a number of single items assessing additional symptoms (dyspnea, sleep disturbance, constipation and diarrhea). The supplemental questionnaire QLQ-LC13 contains 13 questions concerning symptoms frequently present in lung cancer patients. All raw scores are linearly converted to a 0 to 100 scale. For the functional and global health status/quality of life scales, higher scores represent a better level of functioning. For the symptom scales, higher scores represent a greater degree of symptoms. Quality of life and respiratory symptoms were assessed before the start of radiotherapy and subsequently 2 weeks, 6 weeks and 3,6 and 12 months after the end of radiotherapy.

After informed consent was obtained, the questionnaire was distributed to the patients who were asked to return the questionnaire within 3 days by mail. During follow up, the 
questionnaires were send to the patients by mail. When the questionnaire was not returned within 4 days, the patient was telephoned and asked to complete and return the questionnaire.

\section{Statistics}

Randomization was carried out centrally by the Comprehensive Cancer Center Limburg in Maastricht, the Netherlands. For randomization, a permuted block design was used. Patients were stratified by institution and external fractionation schedule (radical vs palliative).

The level of palliation of symptoms and QoL was evaluated in two different ways. First, short-term levels of palliation were examined by a subject-specific analysis, assessing response for each symptom and QoL domain in all patients individually. The response rate of symptoms is often calculated only over the subset of patients having a specific symptom at baseline and at least one follow-up assessment. When the follow-up score for a symptom is lower than that reported at baseline, the patient is considered palliated. Conversely, when the follow-up score is higher, the patient is considered to have deteriorated. In the current study we employed an alternative definition of palliation, derived from the proposal of Stephens and co-workers ${ }^{26}$, which also takes into account patients with no or mild symptoms at baseline and those who died before the first follow-up assessment. To assess the response rate for symptoms, each symptom scale was first divided into four categories. For the single-item scales (cough, haemoptysis, pain arm/shoulder, pain chest wall and appetite loss), four ranges of scores were defined: $1=$ nil, $2=$ mild, $3=$ moderate and $4=$ severe. For the multi-item scales (dyspnea and fatigue), four ranges of scores were defined as well, based on the converted score ranging from 0 to $100: 0=$ nil, 1 to $34=$ mild, 35 to 67 $=$ moderate and 68 to $100=$ severe.

The criteria used to define palliation of symptoms, functioning scales and global QoL are described earlier ${ }^{27}$ and are outlined in appendix 1 and 2.

Differences in response rates were tested for statistical significance with a chi-square test.

Secondly, long term results were evaluated by a group-based analysis, comparing changes in mean scores for the two treatment groups at each time point. Differences between the two arms were tested for statistical significance with a repeated 
measurement ANOVA using a mixed effect modeling procedure, SAS Proc Mixed. In contrast to a "complete cases analysis", the mixed effect modeling retains in the analysis patients who dropout during follow-up. Trends over time for dropouts and complete cases are estimated under the assumption that all patients within the same group have the same change pattern over time. SAS Proc Mixed uses the method of restricted maximum likelihood to estimate the parameters of the model. F-tests are used for testing main effects of group and time, and an interaction effect of group $x$ time.

Survival curves were estimated with the Kaplan-Meier method. The log rank test was used to test the statistical significance of differences between survival curves.

The primary endpoint of the study was the response rate of dyspnea, which was the symptom, considered to benefit most from the addition of EBB. The secondary endpoints were the other respiratory symptoms, QoL, re-expansion of atelectasis, survival and complications. Assuming that the rate of palliation of dyspnea would increase from $50 \%$ to $75 \%$, a total number of 160 patients was necessary with a two-sided type I error of 0.05 and a power of $80 \%$. The analysis was performed on all eligible patients according to the intentto-treat principle.

\section{results}

\section{Patient population}

Between June 1994 and December 1998, 98 patients were randomized into the study. Unfortunately, the study was ended prematurely due to insufficient patient accrual. Of the 98 patients randomized, three were excluded because they did not fulfil the eligibility criteria of the study. The reasons for exclusion were the presence of distant metastases, cervical carcinoma in the history of one patient and no histologic confirmation of the diagnosis NSCLC. The analysis was based on the remaining 95 patients. Of the eligible patients, 47 were randomized to receive XRT alone and 48 to XRT and EBB. The two groups were well balanced concerning baseline clinical characteristics (Table 1). Furthermore, no significant group differences were observed in the baseline symptom and QoL scores (Table 2). In 75 patients $(79 \%)$, the radical fractionation schedule and in 20 patients $(21 \%)$, the palliative schedule was used. An atelectasis before treatment was present in 63 patients $(66 \%)$. 
Table 1: Pre-treatment characteristics of the eligible patients stratified by treatment arm.

\begin{tabular}{|c|c|c|c|c|c|}
\hline \multirow[b]{2}{*}{ Variables } & \multicolumn{4}{|c|}{ Treatment arm } & \multirow[b]{2}{*}{ P-value } \\
\hline & \multicolumn{2}{|c|}{$\underset{\substack{\text { XRT } \\
(n=47)}}{ }$} & \multicolumn{2}{|c|}{$\begin{array}{c}X R T+E B B \\
(n=48)\end{array}$} & \\
\hline Sex & & & & & $p=0.75$ \\
\hline Male & 38 & $81 \%$ & 40 & $83 \%$ & \\
\hline Female & 9 & $19 \%$ & 8 & $17 \%$ & \\
\hline Age (mean) & 68 & SD 9 & 67 & SD 9 & $p=0.61$ \\
\hline T-classification & & & & & $p=0.55$ \\
\hline $\mathrm{T} 2$ & 16 & $34 \%$ & 17 & $35 \%$ & \\
\hline T3 & 8 & $17 \%$ & 12 & $25 \%$ & \\
\hline T4 & 23 & $49 \%$ & 19 & $40 \%$ & \\
\hline $\mathrm{N}$-classification & & & & & $p=0.63$ \\
\hline No & 17 & $36 \%$ & 13 & $27 \%$ & \\
\hline N2 & 21 & $45 \%$ & 25 & $52 \%$ & \\
\hline N3 & 9 & $19 \%$ & 10 & $21 \%$ & \\
\hline WHO performance status & & & & & $p=0.80$ \\
\hline WHO 0 & 11 & $11 \%$ & 12 & $25 \%$ & \\
\hline WHO 1 & 26 & $55 \%$ & 28 & $58 \%$ & \\
\hline WHO 2 & 8 & $17 \%$ & 5 & $10 \%$ & \\
\hline WHO 3 & 2 & $4 \%$ & 3 & $6 \%$ & \\
\hline Total dose external irradie & & & & & $p=0.65$ \\
\hline $30 \mathrm{~Gy}$ & 38 & $81 \%$ & 37 & $77 \%$ & \\
\hline $60 \mathrm{~Gy}$ & 9 & $19 \%$ & 11 & $23 \%$ & \\
\hline Localisation & & & & & $p=0.71$ \\
\hline Right upper lobe & 14 & $29 \%$ & 13 & $27 \%$ & \\
\hline Right middle lobe & 4 & $9 \%$ & 4 & $8 \%$ & \\
\hline Right lower lobe & 2 & $4 \%$ & 4 & $8 \%$ & \\
\hline Right main bronchus & 6 & $13 \%$ & 10 & $21 \%$ & \\
\hline Left upper lobe & 14 & $30 \%$ & 8 & $17 \%$ & \\
\hline Left lower lobe & 4 & $9 \%$ & 6 & $13 \%$ & \\
\hline Left main bronchus & 3 & $6 \%$ & 3 & $6 \%$ & \\
\hline Atelectasis & & & & & $p=0.17$ \\
\hline Yes & 28 & $60 \%$ & 35 & $73 \%$ & \\
\hline No & 19 & $40 \%$ & 13 & $27 \%$ & \\
\hline \multicolumn{6}{|l|}{ Inspiratory Vital Capacity } \\
\hline Absolute (cc) & 2845 & SD 846 & 2731 & SD 870 & $p=0.53$ \\
\hline Relative (\%) & 73.5 & SD 20.5 & 72.2 & SD 20.3 & $p=0.71$ \\
\hline
\end{tabular}

Note: None of the differences were statistically significant. 
Table 2: Pre-treatment quality of life characteristics of the eligible patients stratified by treatment arm.

\begin{tabular}{|c|c|c|c|c|c|}
\hline \multirow{3}{*}{ Variables } & \multicolumn{4}{|c|}{ Treatment arm } & \multirow{3}{*}{ P-value ${ }^{1}$} \\
\hline & \multicolumn{2}{|c|}{ XRT (n=47) } & \multicolumn{2}{|c|}{$X R T+E B B(n=48)$} & \\
\hline & mean & (SD) & mean & (SD) & \\
\hline \multicolumn{6}{|l|}{ Functioning scales } \\
\hline Physical functioning & 59.0 & $(27.2)$ & 59.1 & $(30.3)$ & $p=0.99$ \\
\hline Role functioning & 55.7 & $(36.1)$ & 58.5 & $(36.6)$ & $p=0.71$ \\
\hline Emotional functioning & 68.8 & $(24.3)$ & 71.4 & $(24.9)$ & $p=0.60$ \\
\hline Cognitive functioning & 82.6 & $(26.4)$ & 88.3 & $(18.4)$ & $p=0.23$ \\
\hline Social functioning & 73.8 & $(25.8)$ & 76.2 & $(28.2)$ & $p=0.68$ \\
\hline Global quality of life & 54.0 & $(23.7)$ & 56.6 & $(23.0)$ & $p=0.60$ \\
\hline \multicolumn{6}{|l|}{ Symptom scales } \\
\hline Cough & 54.5 & $(23.9)$ & 48.6 & $(21.9)$ & $p=0.22$ \\
\hline Haemoptysis & 13.6 & $(24.2)$ & 15.2 & $(23.0)$ & $p=0.75$ \\
\hline Dyspnea & 33.8 & $(22.5)$ & 36.5 & $(28.7)$ & $p=0.62$ \\
\hline Chest pain & 21.2 & $(29.7)$ & 15.2 & $(26.0)$ & $p=0.44$ \\
\hline Pain arm/shoulder & 17.4 & $(28.3)$ & 13.0 & $(24.8)$ & $p=0.31$ \\
\hline Fatigue & 44.9 & (28.9) & 43.0 & $(28.7)$ & $p=0.75$ \\
\hline
\end{tabular}

Note 1: None of the differences were statistically significant.

Note 2: For the functioning scales, higher scores represent better functioning.

Note 3: For the symptom scales, higher scores represent more symptoms.

\section{Compliance to treatment}

In the group of patients randomized to XRT alone, 40 out of 48 patients $(83 \%)$ received the planned dose. Of the remaining eight patients who did not receive the planned dose, seven were treated according to the radical schedule and one according to the palliative schedule. In the group of patients randomized to XRT + EBB, 41 out of 47 patients $(87 \%)$ received the planned dose of external irradiation. Of the six patients who did not receive the planned dose, four were treated according to the radical schedule and two according to the palliative schedule. In this group, 41 patients $(87 \%)$ received the planned two fractions of brachytherapy. Three patients were not treated with brachytherapy at all: two patients refused and one patient experienced deteriorating general condition and bronchoscopy was 
considered too burdensome. Three patients received one fraction of brachytherapy: two patients refused to undergo a second session and one patient experienced deteriorating general condition due to massive malignant pleural effusion. In five patients (11\%) randomized to receive $\mathrm{XRT}$ and $\mathrm{EBB}$, the field arrangements during radiotherapy had to be adjusted because of re-expansion of atelectasis. In all of these five patients, the radiation portals could be reduced.

\section{Compliance with QoL assessments}

Of the 95 patients included in the study, 90 completed a baseline questionnaire (95\%). The five patients without baseline QoL assessment were excluded from further $\mathrm{QoL}$ analysis. Compliance at subsequent assessments was high: $95 \%$ at 2 weeks ( 76 out of 81 patients at risk), $89 \%$ at 6 weeks ( 66 out of 74 patients at risk), $94 \%$ at 3 months (59 out of 63 patients at risk), $93 \%$ at six months ( 41 out of 43 patients at risk) and $83 \%$ at 12 months (20 out of 24 patients at risk).

\section{Re-expansion and prevention of atelectasis}

Re-expansion of atelectasis was evaluated in two different ways. First, the inspiratory vital capacity (IVC), expressed as the percentage observed from predicted (IVCr), was measured before and 2 weeks after treatment. Pre- and post-treatment values were available for 32 patients treated with XRT alone and for 31 patients treated with XRT + EBB. After XRT alone, the IVCr decreased from $72 \%$ from predicted to $69 \%$ from predicted. In the group treated with XRT and EBB, the IVCr improved from $68 \%$ from predicted to $78 \%$ from predicted, which was significantly better compared to XRT alone $(p=0.02)$.

Significantly higher rates of radiological re-expansion assessed with chest radiograph and CT scan of the chest were observed with XRT and EBB compared to XRT alone. In the group of patients with an atelectasis before radiotherapy, 9 out of $26(35 \%)$ improved with XRT alone, while 17 out of $30(57 \%)$ improved with XRT and EBB (Table 3). Among patients without atelectasis before radiotherapy, progression was observed in none of the patients randomized for XRT and EBB, while three patients became progressive after XRT alone (Table 3). 
Table 3: Response and prevention of atelectasis stratified by treatment arm.

\begin{tabular}{|c|c|c|c|c|c|}
\hline \multirow{3}{*}{ Response } & \multicolumn{4}{|c|}{ Treatment arm } & \multirow{3}{*}{ P-value } \\
\hline & \multicolumn{2}{|c|}{ XRT $(n=47)$} & \multicolumn{2}{|c|}{$X R T+\operatorname{EBB}(n=48)$} & \\
\hline & number & $\%$ & number & $\%$ & \\
\hline \multicolumn{5}{|l|}{ Atelectasis before radiotherapy } & $p=0.009$ \\
\hline Complete disappearance & 0 & $0 \%$ & 8 & $27 \%$ & \\
\hline Partial disappearance & 9 & $35 \%$ & 9 & $30 \%$ & \\
\hline No change & 11 & $42 \%$ & 9 & $30 \%$ & \\
\hline Progression & 6 & $23 \%$ & 4 & $13 \%$ & \\
\hline Not evaluable & 2 & & 5 & & \\
\hline \multicolumn{5}{|c|}{ No atelectasis before radiotherapy } & $p=0.11$ \\
\hline Prevention & 8 & $67 \%$ & 8 & $100 \%$ & \\
\hline Progression & 4 & $33 \%$ & 0 & $0 \%$ & \\
\hline Not evaluable & 7 & & 5 & & \\
\hline
\end{tabular}

Table 4: Response classification for dyspnea stratified by treatment arm

\begin{tabular}{lccccc}
\hline & \multicolumn{5}{c}{ Treatment arm } \\
\cline { 2 - 3 } Response classification & \multicolumn{2}{c}{ XRT $(n=44)^{*}$} & & \multicolumn{2}{c}{ XRT + EBB $(n=39)^{*}$} \\
\cline { 2 - 3 } \cline { 5 - 6 } & number & $\%$ & & number & $\%$ \\
\hline Response & 16 & $36 \%$ & & 18 & $46 \%$ \\
$\quad$ Improvement & 7 & $16 \%$ & & 9 & $23 \%$ \\
Control & 7 & $16 \%$ & & 6 & $15 \%$ \\
Prevention & 2 & $5 \%$ & & 3 & $8 \%$ \\
No response & 19 & $43 \%$ & & 16 & $41 \%$ \\
$\quad$ No response & 9 & $21 \%$ & & 10 & $26 \%$ \\
$\quad$ Dead without palliation & 10 & $23 \%$ & & 6 & $15 \%$ \\
Worse & 9 & $21 \%$ & & 5 & $13 \%$ \\
\hline
\end{tabular}

* The number between brackets refer to the number of evaluable patients 


\section{Response of dyspnea}

Among patients allocated to receive XRT alone, the response rate for dyspnea was $37 \%$ (16 out of 43 ) compared to $46 \%$ (18/39) among those allocated to receive XRT + EBB, which was not statistically significant $(p=0.29)$. In the XRT-alone arm, $42 \%$ were classified as 'no response' and $21 \%$ as 'progressive', compared to $41 \%$ and $13 \%$ respectively in the XRT+EBB arm (Table 4).

\section{Changes in mean scores for dyspnea}

Analyzing differences of the mean scores, a significant difference between the two treatment arms was observed with regard to dyspnea (Figure 1). After XRT alone, dyspnea became worse at the first follow up time point ( 2 weeks) and continued to worsen with time. After XRT and EBB, a temporary improvement in dyspnea was observed which disappeared after 3 months $(p=0.02)$. The results regarding dyspnea were further analyzed separately in the subset of patients with obstructive tumor in the main bronchus and in the subset of patients with obstructive tumor in the lobar bronchus (Figure 2). A striking difference was observed in the former group but not in the latter group. The level of improvement of dyspnea was highest among those with a complete re-expansion of

Figure 1: Change scores for dyspnea over time, stratified by treatment arm.

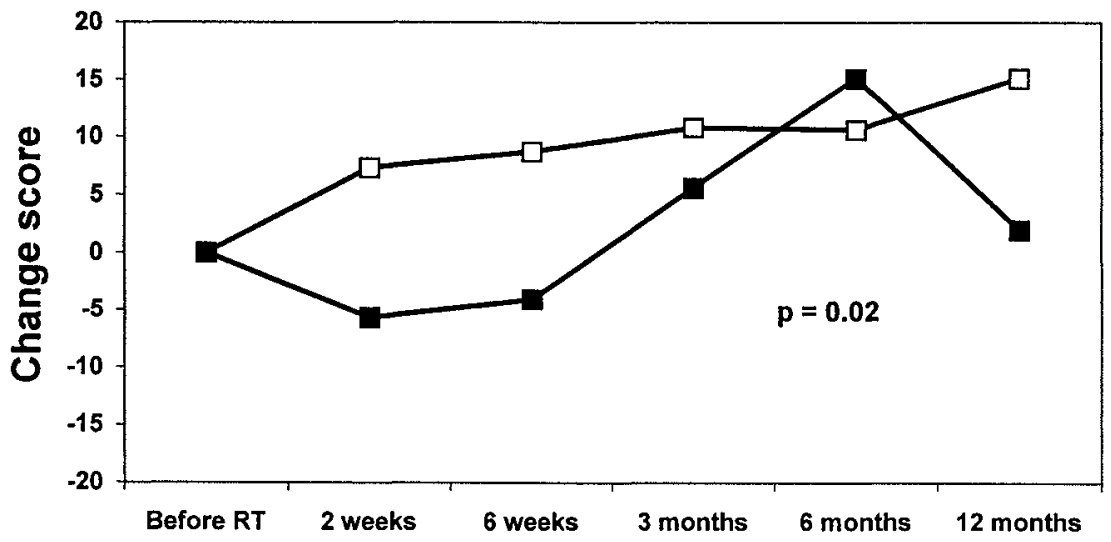

Note: A positive difference score represents an increase and a negative score a decrease of symptoms. 
collapsed lung and worsened in those with progression of atelectasis (Figure 3).

\section{Response of other respiratory symptoms and QoL}

The response rates for the other respiratory symptoms did not differ significantly between the XRT-alone group and the XRT+EBB group. For cough, the response rates were $38 \%$ versus $24 \%$, respectively, $82 \%$ versus $86 \%$ for haemoptysis, $67 \%$ versus $80 \%$ for chest pain and $69 \%$ versus $74 \%$ for pain in the arm/shoulder. No significant differences were noted in response rates for any of the functioning scales and the global QoL scale.

Changes in mean scores of symptoms and quality of life

There was a tendency towards worse palliation of cough and chest pain after XRT and EBB compared to XRT alone, however, these differences did not reach conventional levels of statistical significance. No significant group differences were observed for the other symptoms or for the functioning and global QoL scales.

\section{Survival, causes of death and complications}

The median survival after XRT alone was 8.5 months (95\%-ci: 5.4-11.6 months) and for XRT and EBB 7.0 months (95\%-ci: 5.3-8.9) (Figure 4) ( $p=0.21$ ). The causes of death are shown in Table 5. No significant differences were observed between the two treatment groups. In particular, six patients treated with XRT died of massive haemoptysis (13\%) compared to seven patients treated with XRT and EBB (15\%). One of these patients who died from massive haemoptysis and who was treated with XRT and EBB, also developed a broncho-esofageal fistula two weeks before massive lung bleeding. Furthermore, in this treatment group, one patient developed a broncho-pleural fistula approximately 9 months after radiotherapy. 
Figure 2: Change scores for dyspnea for obstructing tumours in the lobar bronchus and main bronchus.

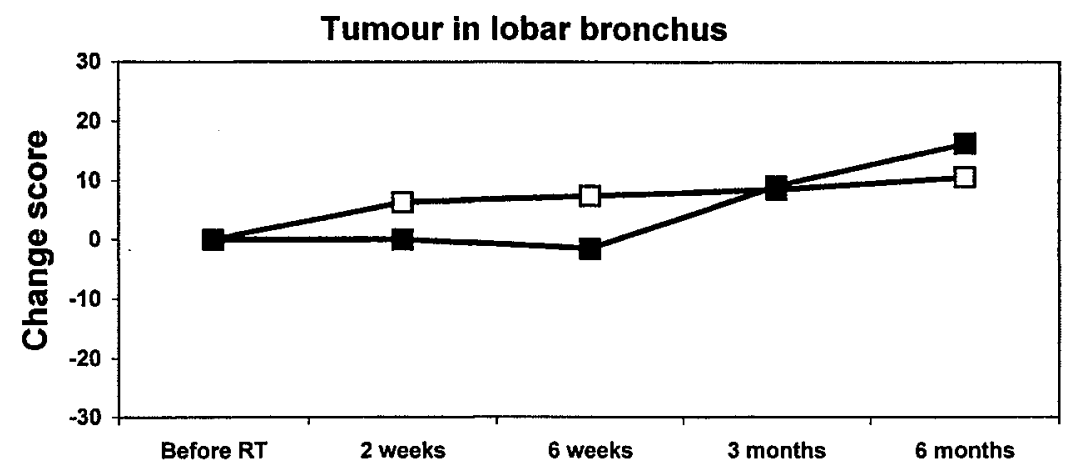

Tumour in main bronchus

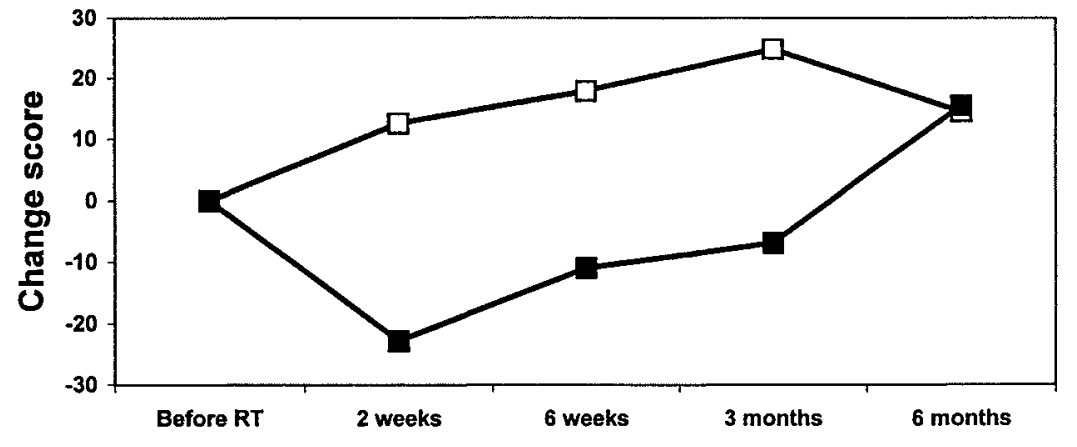

\section{XRT}

\section{XRT+EBB}

Note: A positive difference score represents an increase and a negative score a decrease of symptoms.

\section{Discussion}

In the present study, the additional value of early concomitant EBB during XRT was investigated with a prospective randomized design. The two treatment groups were well balanced concerning pre-treatment characteristics. The first hypothesis to be tested was whether the addition of EBB to XRT offered better rates of re-expansion of collapsed lung. Both methods used to evaluate this, i.e. radiological re-expansion and improvement of the 
Figure 2: Change scores for dyspnea for obstructing tumours in the lobar bronchus and main bronchus.
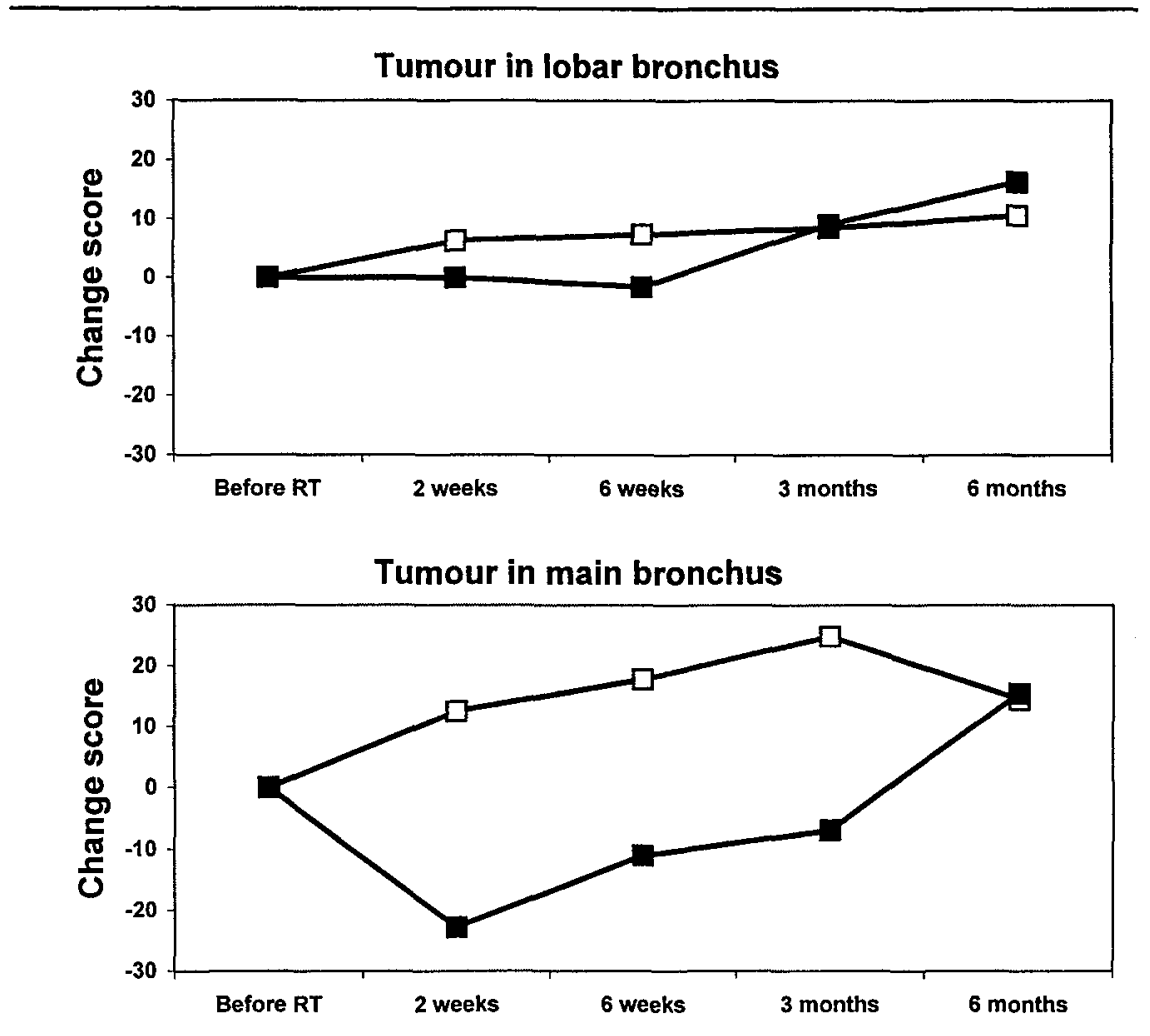

\section{XRT}

XRT+EBB

Note: A positive difference score represents an increase and a negative score a decrease of symptoms.

inspiratory vital capacity showed that the level of improvement was significantly higher when XRT was combined with EBB as compared to XRT alone. In addition, a significantly greater degree of improvement regarding the mean values of dyspnea was achieved by the addition of concomitant EBB during XRT. This beneficial effect lasted for only 6 weeks and disappeared after three months. Furthermore, the beneficial effect on dyspnea did not translate into an improvement in functioning or global QoL. There has been some concern that improving ventilation areas that are not perfused because of malignant infiltration of the pulmonary vessels could increase the physiological dead space and lead to worsening of 
Figure 3: Change scores for dyspnea over time, stratified by re-expansion of collapsed lung.

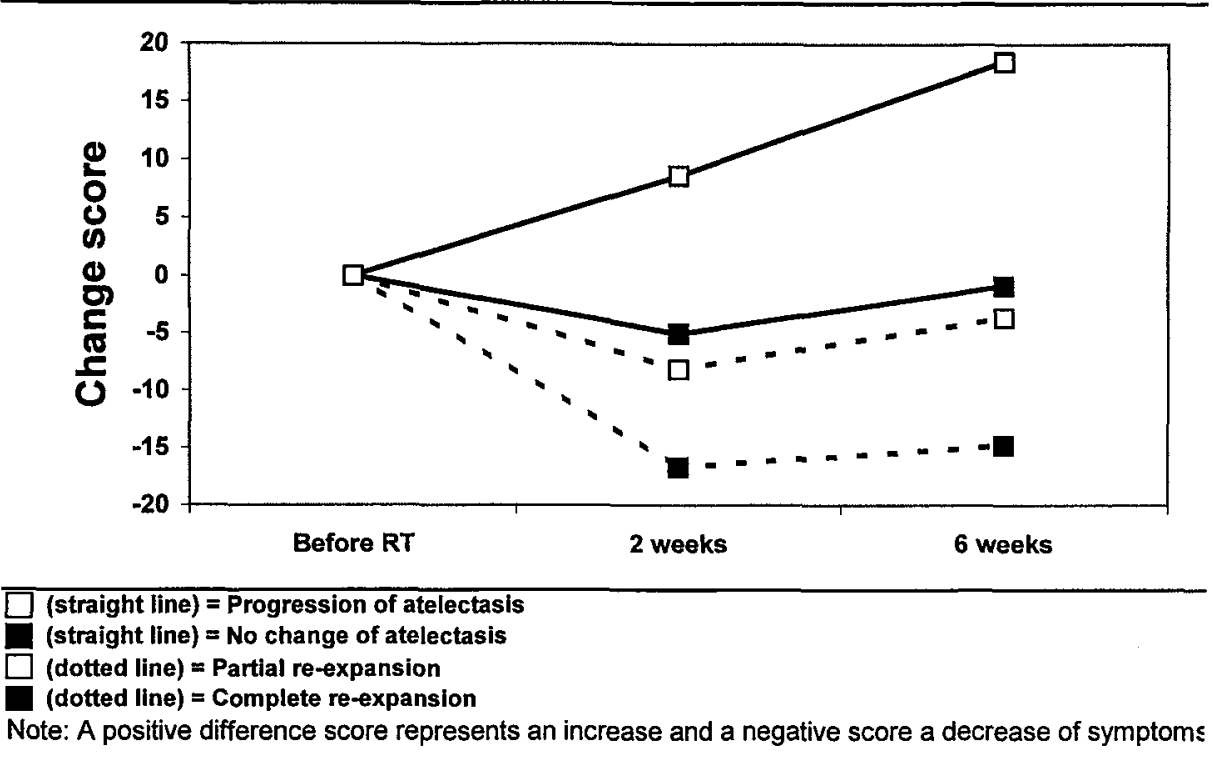

dyspnea. However, in the present study, patients with a complete re-expansion showed the best improvement in the level of palliation of dyspnea, and no difference was noted between those with a partial re-expansion and no change of collapsed lung. Goldman and coworkers ${ }^{28}$ investigated physiological changes among patients treated with HDR EBB for major airway occlusion by malignant diseases. They observed a significant increase in fractional ventilation and perfusion for the affected lung and no worsening of shortness of breath in case of re-expansion. These findings suggest that re-expansion of collapsed lung does not necessarily lead to worsening of dyspnea, and may actually improve dyspnea in case of a complete re-expansion. A striking finding was that the difference in palliation of dyspnea in favor of the patients randomized for XRT and EBB was only observed in the subset of patients with a tumor obstructing the main bronchus, and not in the patients with obstructing tumors in the lobar bronchus. This finding is supported by the report of Goldman and co-workers ${ }^{28}$. They found that patients with tumors occluding a main bronchus had greater improvement in physiological indices, including spirometric indices, fractional ventilation and perfusion, and five minute walking distance, than those who had a tumor in a lobar bronchus. In the latter group, no improvement was observed for any of the 
physiological indices mentioned. It has to be emphasized that no definitive conclusions can be drawn from this subset analysis. However, these findings stress the need for further prospective evaluation in the group of patients with obstructing endobronchial tumor in the main bronchus.

Although, in the analysis comparing mean scores a significant difference between the two treatment arms was observed regarding dyspnea, no such difference was noted in the analysis comparing response rates for dyspnea. This apparent inconsistency could be explained by the fact that 'improvement' of a symptom was defined as a decrease on two consecutive assessments in the first 3 months after therapy. The same holds for the definition of 'control' and 'prevention'. As the beneficial effect in favor of EBB and XRT tended to disappear at 3 months, few patients experienced a response on at least two consecutive assessments.

One of the shortcomings of the current study is that it ended prematurely due to a decreasing accrual of patients. The main reason for this decreasing accrual was the increasing use of chemotherapy, which is now currently used in the majority of patients with locally advanced NSCLC. Patients with prior or planned chemotherapy were ineligible for the current study. The limited number of patients entered in the present study raises the question whether there was sufficient power to detect differences if they were actually there (i.e. protecting against type II errors).

Massive haemoptysis has frequently been described as a major complication after EBB. The results of a previous analysis indicated that the relatively high incidence of massive haemoptysis observed among patients treated with EBB was probably due to selection of patients and not to EBB itself [Langendijk]. In that study, a dramatic increase in the incidence of massive haemoptysis was only observed among those patients treated with XRT in combination with a single fraction of $15 \mathrm{~Gy}$. The incidence of massive haemoptysis of those treated with XRT and EBB with two fraction of 7.5 Gy was similar to that observed in the group of patients treated with XRT alone but who would have been eligible for EBB. In the current prospective randomized study, the findings of the retrospective study were confirmed; no difference was noted in the incidence of massive haemoptysis between the two treatment arms. Huber and co-workers reported on the results of another prospective study in which patients were randomized between XRT (60 Gy) and XRT (60 Gy) and two session of EBB with 4.8 Gy applied 1 week before and 3 weeks after completion of XRT. 
The incidence of massive haemoptysis was $15.4 \%$ after XRT alone and $20.8 \%$ after XRT plus EBB; a difference which was not statistically significant ${ }^{29}$. These findings indicate that the addition of EBB to XRT does not lead to an increase of massive haemoptysis.

The survival rate in the group of patients randomized to receive XRT and EBB was not significantly different from those randomized to XRT alone, although, the curves diverged after 6 months. We could not find an explanation for this finding. The incidence of major complications was similar and the same holds for the other causes of death.

In conclusion, concomitant EBB during XRT provides higher response rates for reexpansion of collapsed lung compared to XRT alone. A temporary but significant higher level of palliation of dyspnea was observed with additional EBB. However, the findings suggest that this beneficial effect of additional EBB is confined to the group of patients with obstructing tumors of the main bronchus, but this needs further investigation. 


\section{$\square$ References}

1. Medical Research Council Lung Cancer Working Party. A Medical Research Counsel (MRC) randomized trials of palliative radiotherapy with two fractions or a single fraction in patients with inoperable non-small cell lung cancer (NSCLC) and poor performance status. Br J Cancer 1992; 65: 934-941.

2. Medical Research Council Lung Cancer Working Party. Inoperable non-small cell lung cancer (NSCLC): a Medical Research Counsel randomized trial of palliative radiotherapy with two or ten fractions. $\mathrm{Br} J$ Cancer 1991; 63: 265270.

3. Lutz ST, Huang DT, Ferguson CL, et al. A retrospective quality of life analysis using the lung cancer symptom scale in patients treated with palliative radiotherapy for advanced nonsmall cell lung cancer. Int $\mathrm{J}$ Radiat Oncol Biol Phys 1997; 37: 117-122.

4. Rees GJG, Devrell CE, Barley VL, and Newman HFV. Palliative radiotherapy for lung cancer: two versus five fractions. Clin Oncol 1997; 9: 90-95.

5. Burt $P A, O$ 'Driscoll $B R$, Notley $M$, et al. Intraluminal irradiation for the palliation of lung cancer with high dose rate micro-Selectron. Thorax 1990; 45: 765-768.

6. Chang $\mathrm{LL}$, Horvath $\mathrm{J}$, Peyton $\mathrm{W}$, and Ling $\mathrm{S}$. High dose rate afterloading intraluminal brachytherapy in malignant airway obstruction of lung cancer. Int J Radiat Oncol Biol Phys 1994; 28(3): 589-596.

7. Gollins SW, Burt PA, Barber PV, and Stout R. High dose rate intraluminal radiotherapy for carcinoma of the bronchus: outcome of treatment of 406 patients. Radiot Oncol 1994; 33: 31-40.

8. Huber $\mathrm{RM}$, Fischer $\mathrm{R}$, Hautman $\mathrm{H}$, et al. Palliative endobronchial brachytherapy for central lung tumors. A prospective, randomized comparison of two fractionation schedules. Chest 1995; 107: 463-470.

9. Mehta MP, Shahabi S, Jarjour NN, and Kinsella TJ. Endobronchial irradiation for malignant airway obstruction. Int J Radiat Oncol Biol Phys 1989; 17: 847-851.

10. Nori, $D$, Allison $R$, Kaplan $B$, ef al. High dose-rate intraluminal irradiation in bronchogenic carcinoma. Chest 1993; 104: 1006-1011.

11. Speiser BL and Spratling L. Remote afterloading brachytherapy for the local control of endobronchial carcinoma. Int J Radiat Oncol Biol Phys 1993; 25: 579-587.

12. Bedwinek J, Petty A, Bruton $C$, Sofield J, Lee $L$. The use of high dose rate endobronchial brachytherapy to palliate symptomatic endobronchial recurrence of previously irradiated bronchogenic carcinoma. Int J Radiat Oncol Biol Phys 1991; 22: 23-30.

13. Gauwitz $M$, Ellerbroek $N$, Komaki $R$, et al. High dose endobronchial irradiation in recurrent bronchogenic carcinoma. Int J Radiat Oncol Biol Phys 1992; 23: 397-400.

14. Hernandez $P$, Gursahaney $A$, Roman $T$ et al. High dose rate brachytherapy for the local control of endobronchial carcinoma following external irradiation. Thorax 1996; 51: 354-358.

15. Micke $O$, Prott FJ, Schafer $U$, et al. Endoluminal HDR-brachytherapy as a paliiative treatment of patients with recurrent previously irradiated non-small cell lung carcinoma. Strahlenther Onkol 1995; 171: 554-559.

16. Pisch, J, Villamena PC, Harvey JC, et al. High dose-rate endobronchial irradiation in malignant airway obstruction. Chest 1993; 104: 721-725.

17. Seagren SL, Harrell $\mathrm{JH}$, and Horn RA. High dose rate intraluminal irradiation in recurrent endobronchial carcinoma. Chest 1985; 88: 810-814.

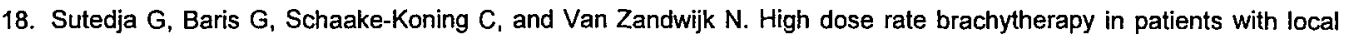
recurrences after radiotherapy of non-small cell lung cancer. Int J Radiat Oncol Biol Phys 1992; 24: 551-553. 
19. Bastin KT, Mehta MP, and Kinsella TJ. Thoracic volume radiation sparing following endobronchial brachytherapy: a quantitative analysis. Int J Radiat Oncol Biol Phys 1993; 25: 703-707.

20. Cotter GW, Lariscy $C$, Ellingwood KE, and Herbert $D$. Inoperable endobronchial obstructing lung cancer treated with combined endobronchial and external beam irradiation: a dosimetric analysis. Int J Radiat Oncol Biol Phys 1993; 27: 531-535.

21. Majid OA, Lee $S$, Khushalani $S$, and Seydel HG. The response of atelectasis from lung cancer to radiation therapy. Int J Radiat Oncol Biol Phys 1986; 12: 231-232.

22. Chetty KG, Moran EM, Sassoon CSH, et al. Effect of radiation therapy on bronchial obstruction due to bronchogenic carcinoma. Chest 1989; 95: 582-584.

23. Slawson RG and Scott RM. Radiation therapy in bronchogenic carcinoma. Ther Radiol 1979; 132: 175-176. In Hermanek, P. and Sobin, L.H. (Ed) In: TNM Atlas: Classification of malignant tumors; Springer-Verlag, 75-84; 1992.

24. Aaronson NK, Ahmedzai S, Bergman B, et al. The European Organization for Research and Treatment of Cancer QLQ-C30: a quality of life instrument for use in international clinical trials in oncology. J Natl Cancer Inst 1993; 85: 365-376.

25. Bergman B, Aaronson NK, Ahmedzai S, et al. The EORTC QLQ-LC13: a modular supplement to the EORTC Core Quality of Life Questionnaire (QLQ-C30) for use in lung cancer clinical trials in oncology. Eur J Cancer 1994; 30A: 635-642.

26. Stephens RJ, Hopwood $P$, and Girting DJ. Defining and analysing symptom palliation in cancer clinical trials: a deceptively difficult exercise. Br J Cancer 1999; 79: 538-544.

27. Langendijk JA, ten Velde GPM, Aaronson NK, et al. Quality of life after palliative radiotherapy in non-small cell lung cancer: a prospective study int J Radiat Oncol Biol Phys 2000; 47, 149-155.

28. Goldman JM, Bulman AS, Rathmell AJ, et al. Physiological effect of endobronchial radiotherapy in patients with major airway occlusion by carcinoma. Thorax 1993; 48: 110-114. 


\section{Appendix 1}

\section{Response criteria for general and respiratory symptoms}

\section{'Response' is defined as:}

- Baseline score 'moderate or severe', with improvement to 'mild' or ' $n i l$ ' $t=$ 'Improvement'.

- Baseline score 'mild', with improvement to 'nil' $t=$ 'improvement'.

- Baseline score 'mild', with 'mild' $\dagger=$ 'control'.

- Baseline score 'nil', with 'nil' $t=$ 'prevention'.

'No response' is defined as:

- Baseline score 'moderate or severe', without improvement to 'mild or nil' $\dagger=$ 'no change'.

- Baseline score 'moderate or severe' and dead before first assessment after the end of radiotherapy = 'dead without palliation'.

- Baseline score 'moderate or severe' without improvement to 'mild or nil' on the first assessment and dead before the second assessment after the end of radiotherapy = 'dead without palliation'.

- Baseline score 'mild', without improvement to 'nil' and without deterioration to 'moderate or severe' $\dagger=$ 'no change'.

- Baseline score ' $m$ ild' and dead before first assessment after the end of radiotherapy = 'dead without palliation'.

- Baseline score 'mild', without improvement to 'nil' on the first assessment and dead before first assessment after the end of radiotherapy $=$ 'dead without palliation'.

- Baseline score ' $n$ il', without ' $n$ il' and without deterioration to 'mild, moderate or severe' $\dagger=$ 'no change'.

- Baseline score 'nil', with deterioration to 'mild, moderate or severe' on the first assessment and dead before the second assessment after the end of radiotherapy = 'dead without palliation'.

'Progression' is defined as:

- Baseline score 'mild', with deterioration to 'moderate or severe' $\uparrow$.

- Baseline score 'nil', with 'mild, moderate or severe' $f$.

'Not evaluable' is defined as:

- Baseline score 'moderate or severe', with improvement to 'nil or mild' on the first assessments and dead before the second assessment after the end of radiotherapy.

- Baseline score 'mild', with improvement to 'nil' on the first assessment and dead before the second assessment after the end of radiotherapy.

- Baseline score 'nil' and dead before the first assessment after the end of radiotherapy.

- Baseline score 'nil', with 'nil' at the first assessment and dead before the second assessment after the end of radiotherapy. 


\title{
Appendix 2
}

\section{Response criteria for functioning scales and global quality of life}

\author{
'Response' is defined as:
}

- Baseline score '0-59', with improvement of at least 5 points $\dagger$ to a minimal value of $40=$ 'improvement'.

- Baseline score ' $\underline{60-79}$ ', with improvement of at least 5 points $\dagger=$ 'improvement'

- Baseline score ' $60-79$ ', with no change (i.e. $<5$ points) $\dagger=$ 'control'

- Baseline score ' $\underline{80-100}$ ', with improvement or no change (i.e. $<5$ points) $\dagger=$ 'prevention'

\section{'No response' is defined as:}

- Baseline score ' $0-59$ ', with no change (i.e. $<5$ points) $\dagger=$ 'no change'

- Baseline score ' $0-59$ ' and dead before first assessment after the end of radiotherapy = 'dead without palliation'.

- Baseline score ' $\underline{0-59}$ ' without improvement of at least 5 points to a minimal value of 40 on the first assessment and dead before the second assessment after the end of radiotherapy = 'dead without palliation'.

- Baseline score ' $60-79$ ' and dead before first assessment after the end of radiotherapy = 'dead without palliation'.

- Baseline score ' $\underline{60-79}$ ' without improvement of at least 5 points on the first assessment and dead before the second assessment after the end of radiotherapy = 'dead without palliation'.

- Baseline score ' $100-100$ ', and dead before first assessment after the end of radiotherapy = 'dead without palliation'.

- Baseline score ' $\underline{80-100}$ ' without improvement of at least 5 points on the first assessment and dead before the second assessment after the end of radiotherapy = 'dead without palliation'.

\section{'Progression' is defined as:}

- Baseline score ' $0-59$ ', with a decrease of at least 5 points $\dagger$

- Baseline score ' $60-79$ ', with a decrease of at least 5 points $\dagger$

- Baseline score ' $80-100$ ', with a decrease of at least 5 points $\dagger$

\section{'Not evaluable' is defined as:}

- Baseline score ' $\underline{0-59}$, with improvement of at least 5 points to a minimal value of 40 on the first assessments and dead before the second assessment after the end of radiotherapy.

- Baseline score ' $60-79$ ', with improvement of at least 5 points on the first assessment and dead before the second assessment after the end of radiotherapy.

- Baseline score ' $80-100$ ', with improvement of at least 5 points on the first assessment and dead before the second assessment after the end of radiotherapy. 
Chapter 11

General discussion 


\section{Introduction}

The outcome for most patients with inoperable non-small cell lung cancer (NSCLC) is poor. Although radiotherapy has a defined role in the local management of these tumours, most patients eventually will die from loco-regional tumour progression or distant metastases ${ }^{12,38,40}$. Taking into account the relatively poor prognosis in these patients and the minimal gain achieved with new modalities regarding the traditional endpoints, it becomes increasingly important to incorporate quality of life (QoL) as an endpoint into clinical studies. It is surprising how little is known about changes in QoL after radiotherapy for inoperable NSCLC. Therefore, this thesis evaluated various aspects of QoL before, during and after radiotherapy among patients with inoperable NSCLC.

\section{Quality of life assessment}

Over 50 instruments have been developed and used to assess QoL in lung cancer patients ${ }^{33}$. For the purpose of this thesis, it was decided to use the EORTC QLQ-C30 in conjunction with the lung cancer module, the EORTC QLQ-LC13. This QoL life instrument proved to be valid and reliable ${ }^{1,5}$ in international studies, allowed evaluation of general health measures as well as disease-specific and treatment-related symptomatology and was available in the Dutch language. Before the study was started, some doubts were expressed regarding patients' and doctors' compliance in assessing QoL in a group of patients with a relatively short prognosis. To overcome this problem, the datamanagement was organised in such a way, that interference of the treating physicians in the distribution and completion of the follow up questionnaires was minimised. Furthermore, by appointing a specific individual (other than the treating physicians) to collect the QoL data, and by limiting the frequency with which questionnaires were administered, it was possible to achieve a compliance of approximately $90 \%$, even in the group of patients with the shortest survival. It has to be emphasised that those patients who did not complete their questionnaire were generally burdening a deteriorating general condition due to disease progression which is in concordance with reports by other investigators $13,14,17$. Therefore, it is extremely important to achieve high rates of 
compliance because a high rate of missing data will undoubtedly lead to biased results. Of the 282 patients with pathologically confirmed NSCLC referred to the RTIL in the period that patients could be included, 264 (94\%) participated in the study. These high rates of compliance and participation imply that the group of patients investigated might be considered representative for patients with inoperable NSCLC referred and treated with primary radiotherapy.

\section{Considerations regarding the optimal radiotherapeutic treatment} approach in inoperable non-small cell lung cancer.

In the study period, patients were selected for three different fractionation schedules (i.e. the palliative, radical and curative schedule) based on a number of well known prognostic factors, including performance status, disease stage and tumour diameter. Major differences regarding the overall survival were observed between these three prognostic groups. It is most likely that these differences regarding the overall survival result from the selection criteria used but it can not be ruled out that they may also be the result of differences in the total dose administered ${ }^{37}$. One of the problems to which clinicians treating these patients are frequently faced with is how to select patients for the most appropriate treatment approach and more specifically, how to prevent more aggressive treatments for those who might benefit more from a less aggressive approach. On the other hand, since higher doses of radiotherapy ${ }^{37}$ and the addition of chemotherapy ${ }^{48}$ provide a small but significant survival benefit, selecting patients for less aggressive approaches might lead to concessions regarding the survival duration. This dilemma particularly plays a role in the choice between palliative and radical treatment. It is therefore of the utmost importance to identify subsets of patients treated with radical radiotherapy with very poor survival rates.

In the study, investigating the prognostic significance of different QoL domains and other prognostic factors in a group of patients treated according the radical schedule, it was possible to identify a subset of patients with such a poor prognosis that palliative radiotherapy was considered more appropriate. The study population was restricted to the radically treated patients to rule out the possible influence of the radiation dose 
administered. The addition of the QoL domains into the multivariate model, showed that global QoL was the most important prognostic factor in the group of patients treated with radical radiotherapy. The addition of the $\mathrm{QoL}$ domains into the multivariate model also identified a slightly different subset of patients with very poor prognosis. These results indicate that $\mathrm{QoL}$ is not only an outcome determinant after radiotherapy, but also that $\mathrm{QoL}$ is a valuable prognostic indicator. QoL has been recognised as a prognostic factor among patients with NSCLC in a number of studies now. Although these studies show differences concerning the eligibility criteria, the treatment modalities and the instruments used to assess QoL, the results are quite consistent and all point out in the same direction.

One of the reasons to treat patients with relatively favourable prognostic factors with radical radiotherapy is the assumption that higher rates of long-term loco-regional tumour control achieved with higher doses of irradiation might also result in long-term palliation of symptoms and improvement of QoL. The results regarding changes in symptoms and $\mathrm{QOL}$ observed in those patients treated according to the radical schedule show that this is partly true. The mean scores for cough and haemoptysis remained relatively stable up to 12 months after radiotherapy. However, the mean scores for the other respiratory symptoms and general symptoms, including fatigue and appetite loss gradually increased over time. Regarding the functioning scales and global QoL, the mean scores remained rather stable up to 6 months with a sharp deterioration at 12 months.

As pointed out before, the choice for a palliative fractionation schedule might give worse results regarding the duration of survival compared to a radical schedule ${ }^{37}$. The question is whether the choice for a palliative instead of a radical schedule might lead to a compromise regarding relief of symptoms and QoL as well. Reviewing the response rates for the symptoms, functioning scales and global QoL scale achieved with the palliative and radical schedule, there are no apparent differences between the two groups, except for relief of appetite loss. These response rates only represent the changes observed in the first three months after radiotherapy. However, such comparisons have to be interpreted with caution. All patients were selected for and not randomised between the two treatment approaches and major differences existed not only regarding prognostic factors but also regarding the baseline symptoms en QoL scores. Furthermore, because of the lack of a sufficient number of patients in the palliative group with survival rates longer than 3 months, no reliable conclusions can be made regarding the possible changes beyond this 
interval. There are no prospective randomised studies comparing palliative with radical radiotherapy. The studies performed by the Medical Research Council did not show a difference either between conventional palliative fractionation ( $10 \times 3$ Gy or $6 \times 4.5 \mathrm{~Gy}$ ) and a two-fraction regimen $(2 \times 8.5 \mathrm{~Gy}){ }^{5}$, nor between the two-fraction regimen and a single fraction regimen $(1 \times 10 \mathrm{~Gy})^{6}$. However, the patients in these two studies were considered to advanced to be eligible for curative or radical treatment and do not provide information about differences between palliative and radical radiotherapy concerning relief of symptoms and QoL.

Patients with stage I NSCLC who are inoperable for medical reasons are potentially curable with high dose radiotherapy, with 5 -year survival rates varying from $6 \%$ to $27 \%$ $10,11,15,20,21,25,27,34,39,44,50,52$ and are significantly better compared to the more advanced stages. As in these patients the radiation fields portals can be limited to the primary site, curative treatment should be given in these patients provided that their general condition allows a longer course of treatment.

\section{Objective tumour response}

It is extremely difficult to say how the changes in respiratory symptoms observed after radiotherapy should be interpreted exactly. There are a number of factors which might contribute to changes of pulmonary symptoms after radiotherapy, including tumour reduction, re-expansion of collapsed lung, pleural effusion, radiation-induced pulmonary toxicity, pre-existing co-morbidity and eventually tumour progression.

In many studies, tumour response is used a proxy for relief of symptoms and/or improvement of QoL. Before analysing the putative association between objective tumour response and symptom and QoL changes, we were interested to find out whether chest radiograph was a reliable tool to assess tumour response. We found that CT was far superior in picking out measurable disease than chest radiograph. However, in the subset of patients with measurable lesions, the chest radiograph was a useful tool to assess response, particularly in case of peripherally localised tumours without atelectasis. In this subset of patients, the results were similar enough to warrant the use of chest radiograph as a method to evaluate response saving some inconvenience for the patient. Furthermore, 
in an era of cost-consciousness, it is important to realise that despite its imperfections the chest radiograph is an adequate tool in a selected group of patients. Based on the results of this study, we decided to test the possible relationship between tumour response and symptom and QoL response in those patients with measurable disease on chest radiograph and that CT was only performed to assess tumour response in case of non-measurable lesions on chest radiograph.

In the group of patients treated with palliative radiotherapy, tumour reduction appeared to be a mechanism by which relief of some respiratory symptoms, such as cough, haemoptysis and dyspnea was achieved while changes in chest pain and arm/shoulder pain did not depend on objective tumour response. After radical radiotherapy, an association was found between objective tumour response and response of chest pain and arm/shoulder pain. It has to be emphasised that 'no objective tumour response' does not necessarily mean that there is no tumour reduction at all. Tumour response was defined as a reduction of the product of the two largest perpendicular tumour diameters of $50 \%$ or more. In case of 'no tumour response', tumour reduction is still possible up to $50 \%$. The results indicate that reduction of the tumour by radiotherapy plays a limited role in the relief of some respiratory symptoms, but not in improvement of most functioning scales and global QoL. Therefore, objective tumour response should not be used as a proxy for subjective endpoints.

\section{Endobronchial brachytherapy}

The results of the QoL studies in patients treated according to the palliative, radical and curative schedule, all show: (1) that dyspnea is one of the most frequently reported symptoms in inoperable NSCLC; $(2)$ that response rates for dyspnea are relatively low and (3) that there is a gradual increase of dyspnea with time. Furthermore, in the study in which baseline QoL was investigated, dyspnea was the only respiratory symptom effecting all functioning scales, except for emotional functioning, and the global QoL scale. Therefore, it might be very important and clinically relevant to find ways to achieve better palliation of dyspnea. One possible way to achieve this could be the addition of endobronchial brachytherapy $(\mathrm{EBB})$ to external irradiation in patients with obstructing 
tumours in the proximal airways. The development and implementation in clinical practice of a high activity iridium-192 source for use in remote afterloading systems, solved many of the problems associated with EBB. Brachytherapy can be administered now without radiation exposure to the medical staff in a relatively short time and is commonly used in clinical practice, including in lung cancer.

Reports on the combination of EBB and external irradiation suggested a beneficial effect compared to external irradiation alone ${ }^{3,8,9,45}$, but no prospective randomised studies were available. Some radiation-oncologists and pulmonologists were quite reluctant to start a prospective randomised trial because of the high frequency of massive haemoptysis reported after EBB ranging from $0-50 \%$ 2,4,19,22-24,26,28-3135-36,41-43,45,4649,51. Therefore, we decided to perform a retrospective analysis to investigate whether EBB was really a risk factor for massive haemoptysis. This analysis showed that EBB administered concurrently with external irradiation did not lead to a higher risk of massive haemoptysis as compared to external irradiation alone provided that fraction sizes for EBB were used of $7.5 \mathrm{~Gy}$ or 10 Gy. Based on these results, we decided to use two fractions of $7.5 \mathrm{~Gy}$ (at $1 \mathrm{~cm}$ ) for EBB to test the additional value of EBB to external radiotherapy in the prospective randomised study.

The prospective randomised phase III study was conducted to test two specific hypotheses. Firstly, we expected that in patients with centrally localised NSCLC, the addition of concomitant EBB during external irradiation would result in higher rates of reexpansion of post-obstructive infiltration/atelectasis and/or higher rates of prevention of these post-obstructive features. Secondly, we expected that these higher rates of reexpansion would result in higher levels of palliation of respiratory symptoms, in particular for dyspnea. An effect of EBB on functioning and global QoL was expected as well. The addition of EBB to external irradiation provided higher rates of re-expansion of collapsed lung resulting in transient lower levels of dyspnea up to three months after radiotherapy. This effect was only observed in those patients with obstructing tumours located in one of the main bronchi. Unfortunately, this study ended prematurely due to an insufficient accrual of patients. Because of the limited number of patients entered in the present study, conclusions have to be drawn with great caution. Recently, the Medical Research Council reported on a prospective randomised study in which patients with endotracheal or endobronchial obstruction by NSCLC were randomly assigned to receive external 
irradiation alone versus external irradiation plus any form of endobronchial treatment (brachytherapy, laser resection or cryotherapy) ${ }^{32}$. Unfortunately, also this study was ended prematurely after inclusion of 75 of the 400 patients required.

Although a beneficial effect of EBB is suggested in patients with obstructing tumours of the main bronchus, the lack of recruitment in both trials preclude definitive conclusions. The relative advantage of EBB remain to be determined.

\section{Conclusions}

Patients with inoperable NSCLC referred for radiotherapy present with a wide variety of signs and symptoms. One of the aims of radiotherapy is to relief these symptoms and to improve QoL for a certain period of time.

This study shows that assessment of QoL is feasible and that high compliance rates can be achieved even in patients with short survival duration.

The results show that radiotherapy offers excellent palliation of haemoptysis and good palliation of pain, but is less adequate in relieving cough, dyspnea and general symptoms including fatigue and appetite loss. In general, the response rate for different domains of functioning and global QoL is achieved in less than half of the patients.

One of the mechanisms by which relief of symptoms is achieved by radiotherapy is tumour reduction, but it certainly does not explain all the changes in symptoms, functioning and QoL. Therefore, tumour response can not be used as proxy for relief of symptoms and/or improvement of QoL.

Besides that QoL is an important and relevant endpoint after treatment, baseline QoL provides important prognostic information regarding the overall survival.

Endobronchial brachytherapy can be administered safely in combination with external irradiation. Although there appears to be an advantage for EBB regarding re-expansion of collapsed lung and relief of dyspnea in patients with obstructing tumours in the main bronchus, its definitive relative advantage remains to be determined. 


\section{References}

1. Aaronson NK, Ahmedzai S, Bergman $B$, et al. The European Organization for Research and Treatment of Cancer QLQ-C30: a quality of life instrument for use in international clinical trials in oncology. J Natl Cancer Inst 1993; 85: 365-376.

2. Aygun $\mathrm{C}$, Weiner $\mathrm{S}$, Scariato, et al. Treatment of non-small cell lung cancer with external beam radiotherapy and high dose rate brachytherapy. Int J Radiat Oncol Biol Phys 1992; 23: 127-132.

3. Bastin KT, Mehta MP, and Kinsella TJ. Thoracic volume radiation sparing following endobronchial brachytherapy: a quantitative analysis. Int J Radiat Oncol Biol Phys 1993; 25: 703-707.

4. Bedwinek J, Petty $A$, Bruton, $C$, et al. The use of high dose rate endobronchial brachytherapy to palliate symptomatic endobronchial recurrence of previously irradiated bronchogenic carcinoma. Int J Radiat Oncol Biol Phys 1991; 22: 2330 .

5. Bergman B, Aaronson, NK, Ahmedzai S, et al. The EORTC QLQ-LC13: a modular supplement to the EORTC Core Quality of Life Questionnaire (QLQ-C30) for use in lung cancer clinical trials in oncology. Eur J Cancer 1994; 30A: 635-642.

6. Bleehen NM, Girling DJ, Fayers PM, et al. Inoperable non-small lung cancer (NSCLC): a Medical Research Council randomized trial of palliative radiotherapy with two fractions or 10 fractions. $\mathrm{Br} J$ Cancer 1991; 63: 265-270.

7. Bleehen NM, Girling DJ, Machin D, et al. A Medical Research Council (MRC) randomized trial of palliative radiotherapy with two fractions or single fraction in patients with inoperable non-small-cell lung cancer (NSCLC) and poor performance status. $\mathrm{Br} J$ Cancer 1992; 65: 934-941.

8. Chang $L L$, Horvath J, Peyton $W$, and Ling $S$. High dose rate afterloading intraluminal brachytherapy in malignant airway obstruction of lung cancer. Int J Radiat Oncol Biol Phys 1994; 28(3): 589-596.

9. Cotter GW, Lariscy $C$, Ellingwood $K E$, and Herbert D. Inoperable endobronchial obstructing lung cancer treated with combined endobronchial and external beam irradiation: a dosimetric analysis. Int J Radiat Oncol Biol Phys 1993; 27: 531-535.

10. Coy, $P$ and Kennelly, GM The role of curative radiotherapy in the treatment of lung cancer. Cancer 1980; 45: 698702.

11. Dosoretz DE, Galmarini D, Rubenstein $\mathrm{JH}$, et al. Local control in medically inoperable lung cancer: an analysis of its importance in outcome and factors determining the probability of tumor eradication. J Radiat Oncol Biol Phys 1993; 27: 507-516.

12. Eichorn HJ. Different fractionation schedules tested by histological examination of autopsy specimen from lung cancer patients. Br J Radiol 1981; 54: 132-135.

13. Ganz PA, Haskell GM, Figlin RA, et al. Estimating the quality of life in a clinical trial of patients with metastatic lung cancer using the Karnofsky performance status and the Functional Living Index-Cancer. Cancer 1988; 61: 849-856.

14. Ganz PA, Figlin RA, Haskell CM, et al. Supportive care versus supportive care and combination chemotherapy in metastatic non-small cell lung cancer. Does chemotherapy make a difference? Cancer 1989; 63: 1271-1278.

15. Gauden $S$, Ramsay $\mathrm{J}$ and Tripciony $\mathrm{L}$. The curative treatment by radiotherapy alone of stage I non-small cell lung cancer. Chest 1995; 108: 1278-1282.

16. Gauwitz M, Ellerbroek N., Komaki $R$, et al. High dose endobronchial irradiation in recurrent bronchogenic carcinoma. Int J Radiat Oncol Biol Phys 1992; 23: 397-400. 
17. Geddes DM, Dones L, Hill E, et al. Quality of life during chemotherapy for small cell lung cancer: assessment and use of daily diary card in a randomized trial. Eur $\mathrm{J}$ Cancer 1990; 26: 484-492.

18. Goldman JM, Bulman AS, Rathmell AJ, et al. Physiological effect of endobronchial radiotherapy in patients with major airway occlusion by carcinoma. Thorax 1993; 48: 110-114.

19. Gollins SW, Ryder WDJ, Burt PA, et al. Massive haemoptysis death and other morbidity associated with high dose rate radiotherapy for carcinoma of the bronchus. Radiother Oncol 1996; 39: 105-116.

20. Graham $\mathrm{RH}$, Gebski VJ and Langlands $A O$. Radical radiotherapy for early nonsmall cell lung cancer. Int $J$ Radiat Oncol Biol Phys 1995; 31: 261-266.

21. Haffty BG, Goldberg NB, Gerstley J, et al. Results of radical radiation therapy in clinical stage I, technically operable non-small cell lung cancer. Int J Radiat Oncol Biol Phys 1988; 15: 69-73.

22. Hernandez $P$, Gursahaney A, Roman T. et al. High dose rate brachytherapy for the local control of endobronchial carcinoma following external irradiation. Thorax 1996; 51: 354-358.

23. Huber RM, Fischer $R$, Hautmann $H_{1}$ et al. Palliative endobronchial brachytherapy for central lung tumors. A prospective, randomised comparison of two fractionation schedules. Chest 1995; 107 (2): $463-470$.

24. Huber $\mathrm{RM}$, Fischer $\mathrm{R}$, Hautmann $\mathrm{H}$, et al.. Does additional brachytherapy improve the effect of external irradiation? $\mathrm{A}$ prospective, randomised study in central lung tumours. Int J Radiat Oncol Biol Phys 1997; 38 (3): 533-540.

25. Kaskowitz L, Graham MM, Emami $B$, et al. Radiation therapy alone for stage 1 non-small cell lung cancer. J Radiat Oncol Biol Phys 1993; 27: 517-523.

26. Khanavkar B, Stern P, Alberti $W$, and Nakhosteen JA. Complications associated with brachytherapy alone or with laser in lung cancer. Chest 1991; 99: 1062-1065.

27. Krol ADG, Aussems P, Noordijk FM, Hermans J, and Leer JWH. Local irradiation alone for peripheral stage I lung cancer: could we omit the elective regional nodal irradiation? Int J Radiat Oncol Biol Phys 1996; 34: 297-302.

28. Lo TCM, Girshovich L, Healey GA, et al. Low dose rate versus high dose rate intraluminal brachytherapy for malignant endobronchial tumours. . Radiother Oncol 1995; 35: 193-197.

29. Mehta MP, Shahabi S, Jarjour NN, and Kinsella TJ. Endobronchial irradiation for malignant airway obstruction. Int J Radiat Oncol Biol Phys 1989; 17: 847-851.

30. Micke $O$, Prott FJ, Schäfer U, Pötter $R$, and Willich $N$. Endoluminal HDR brachytherapy as a palliative treatment of patients with recurrent previously irradiated non-small cell lung carcinoma. Strahlenther Onkol 1995; 171: 554-559.

31. Miller RR and McGregor DH. Haemorrhage from carcinoma of the lung. Cancer 1980; 46: 200-205.

32. Moghissi $K$, Bond MG, Sambrook RJ, et al. Treatment of endotracheal or endobronchial obstruction by non-small cell lung cancer: lack of patients in an MRC randomized trial leaves key questions unanswered. Medical Research Council Lung Cancer Working Party. Clin Oncol (R Coll Radiol) 1999; 11: 179-183.

33. Montazeri A, Gillis CR, and McEwen J. Quality of life in patients with lug cancer. A review of literature from 1970 to 1995. Chest 1998; 113: 467-481.

34. Noordijk EM, vd Poest Clement, et al. Radiotherapy as an alternative to surgery in elderly patients with resectable lung cancer. Radiot Oncol 1988; 13: 83-89.

35. Nori D, Allison R, Kaplan B, et al. High dose rate intraluminal irradiation in bronchogenic carcinoma. Chest 1993; 104 : 1006-1011.

36. Pearlberg JL, Sandler MA, Kvale P, et al.. Computed-tomographic and conventional linear tomographic evaluation of tracheobronchial lesions for laser photoresection. Radiology 1985; 154: 759-762.

37. Perez CA, Stanley K, Grundy G, et al. Impact of irradiation technique and tumour extend in tumour control and survival of patients with unresectable non-oat cell carcinoma of the lung. Cancer 1982; 50: 1091-1099. 
38. Rissanen PM, Tikha $U$, and Holsti RR. Autopsy findings in lung cancer treatment with megavoltage radiotherapy. Acta Radiol Ther 1968; 7, 433-442.

39. Sandler HM, Curran Jr WR and Tunisi III AT. The infiuence of tumor size and pre-treatment staging on outcome following radiation therapy alone for stage I non-small cell lung cancer. Radiat Oncol Biol Phys 1990; 19: 9-13.

40. Saunders MI, Bennet MA, and Dische S. Primary tumor control after radiotherapy for carcinoma of the bronchus. Int J Radiat Oncol Biol Phys 1984; 10: 499-501.

41. Schraube $P, F r i t z, P, B e c k e r ~ H D$ and Wannenmacher $M$. Results of Endoluminal high-dose-rate irradiation of central non-small cell lung cancer. Strahlenther Onkol 1993; 169: 228-234.

42. Schray M, McDougall JC, Martinez A, Cortese DA. and Brutinel WM. Management of malignant airway compromise with laser and low dose rate brachytherapy. Chest 1988; 93: 264-269.

43. Seagren SL, Harrell JH and Hom RA. High dose rate intraluminal irradiation in recurrent endobronchial carcinoma. Chest 1985; 88 (6): 810-814.

44. Slotman BJ, Anthonisse IE, Njo KH. Limited field irradiation in early stage (T1-2NO) non-small cell lung cancer. Radiot Oncol 1996; 41: 41-44.

45. Speiser BL and Spratling L. Remote afterloading brachytherapy for the local control of endobronchial carcinoma. Int J Radiat Oncol Biol Phys 1993; 25: 579-587.

46. Speiser BL and Spratling L. Radiation bronchitis and stenosis secondary to high dose rate endobronchial irradiation. Int J Radiat Oncol Biol Phys 1993; 25: 589-597.

47. Stephens RJ, Hopwood P, and Girling DJ. Defining and analysing symptom palliation in cancer clinical trials: a deceptively difficult exercise. Br J Cancer 1999; 79: 538-544.

48. Stewart LA, and Pignon JP. Chemotherapy in non-small cell lung cancer: a meta-analysis using updated data on individual patients from 52 randomized clinical trials. BNIJ 1995; 311: 899-909.

49. Sutedja G, Baris G, Schaake-Koning $C$ and van Zandwijk N. High dose rate brachytherapy in patients with local recurrences after radiotherapy of non-small cell lung cancer. Int $J$ Radiat Oncol Biol Phys 1992; 24: 551-553.

50. Talton BM, Constable WC and Kersh CR. Curative radiotherapy in non-small cell carcinoma of the lung. Int J Radiat Oncol Biol Phys 1990; 19: 15-21.

51. Zajac AJ, Kohn ML, Heiser $D$ and Peters JW. High-dose-rate intraluminal brachytherapy in the treatment of endobronchial malignancy. Radiology 1993; 187: 571-575.

52. Zhang $H X$, Yin WB, Zhang LJ, et al. Curative radiotherapy of early operable non-small cell lung cancer. Radiot Oncol 1989; 14: 89-94. 


\section{Summary}




\section{Chapter 1}

In chapter 1, the general introduction, an overview is presented concerning general aspects of the assessment and evaluation of quality of life in general and in lung cancer patients specifically. Despite the relatively poor prognosis in inoperable non-small cell lung cancer (NSCLC), information in this group of patients concerning quality of life (QoL) assessed with validated and reliable instruments is very limited.

\section{Chapter 2}

Chapter 2 describes the aims of the thesis and the design of the study. The aims of this thesis were (1) to describe prospectively QoL before and after radiotherapy for inoperable NSCLC treated with radiotherapy, (2) to evaluate the prognostic significance of pre-treatment QoL, (3) the determine which factors influence the changes in QoL and (4) to assess the safety and value of the addition of endobronchial brachytherapy (EBB) to external irradiation (XRT). Therefore, a prospective non-randomised study was initiated in which 264 patients were included. Based on well-defined prognostic factors, these patients were divided into three prognostic groups and subsequently treated according to three different fractionation schedules, i.e. the palliative, radical and curative schedule. In all these patients, QoL was assessed before and during radiotherapy and 2 weeks, 6 weeks and 3, 6, and 12 months after radiotherapy, using he EORTC QLQ-C30 and the lung cancer module QLQ-LC13. To assess the most optimal fractionation schedule for $E B B$, a retrospective analysis among 936 NSCLC patients was performed. To determine the additional value of EBB, a prospective randomised study was initiated, in which patients with centrally localised tumours were randomised between XRT alone versus XRT and EBB.

\section{Chapter 3}

In this chapter, we described pre-treatment QoL characteristics in three prognostic groups of patients with NSCLC referred for radiotherapy. In the group with the poorest prognosis (palliative group), significantly higher scores for pulmonary and general symptoms were reported compared to the groups consisting of patients considered to have a better prognosis (radical and curative group). Secondly, the association between the most important prognostic factors in NSCLC and self-reported pre-treatment QoL was investigated. A significant association was found between WHO performance status and pre-treatment QoL. 
However, the effect sizes for the different QoL domains were rather low, indicating that only a small proportion of the variance observed in the mean QoL scores could be explained by variation in performance status. Weight loss was also significantly associated with physical and social functioning, as well as with fatigue and appetite loss. Surprisingly, no significant association was observed between tumour extension and self-reported QoL. Finally, the impact of the presence and severity of respiratory symptoms on general symptoms and QoL was investigated. Only dyspnea was significantly associated with the EORTC functioning scales and the global QoL scale.

\section{Chapter 4}

In chapter 4 , the results were presented of a prospective study investigating the prognostic significance of pre-treatment QoL among patients with inoperable NSCLC treated according to the radical fractionation schedule. In a multivariate model including only the non-QoL parameters, WHO performance status (WHO 0 versus WHO>0), weight loss $(0-10 \%$ versus $>10 \%$ ) and $\mathrm{N}$-classification (NO versus $\mathrm{N}+$ ) were independent prognosticators for the overall survival. After entering the QoL parameters in the model, global QoL was the strongest prognostic factor, while performance status lost its significance. However, this effect was only observed in those patients with lymph node metastases and not in the clinically NO patients. By combining a number of prognostic factors, a subset of patients could be identified with such a poor prognosis that palliative radiotherapy was considered more appropriate.

\section{Chapter 5}

In chapter 5 , the results of a prospective study are presented which was performed to evaluate whether the chest radiograph was a reliable tool to assess response to radiotherapy. CT was more suited for tumour measurement because more measurable lesions could be found and more evaluable lesions on chest radiograph become measurable on $\mathrm{CT}$. The chest radiograph does have a valuable role to play in those lesions that are measurable because of the good interobserver agreement with regard to the response classification, the high overall agreement between $C T$ and chest radiograph in case of measurable cases and the lack of important differences with regard to the survival. Based on the results of this study, it was decided to assess objective tumour response only with $\mathrm{CT}$ in case of non-measurable lesions on the chest radiograph. 


\section{Chapter 6}

In this chapter, the changes of QoL observed after palliative radiotherapy were described. The QoL response rates were $79 \%$ for haemoptysis, $56 \%$ for arm/shoulder pain, $53 \%$ for chest pain, $49 \%$ for cough and $39 \%$ for dyspnea. Minimal response rates were observed for the general symptoms fatigue and appetite loss. The QoL response rates for the five functioning scales of the QLQ-C30 varied from $35 \%$ for role functioning to $57 \%$ for emotional functioning. Global QoL improved in $37 \%$ of the cases. In general, there was a tendency for better palliation of symptoms and improvement of QoL among patients with an objective tumour response than among those without objective tumour response.

\section{- Chapter 7}

In chapter 7, the changes of QoL observed after radical radiotherapy were presented. The response rates were excellent for haemoptysis $(83 \%)$, good for chest pain $(68 \%)$, arm/shoulder pain (63\%) and appetite loss (59\%) and poor for dyspnea (37\%), cough (31\%) and fatigue (28\%). The QoL response rates for the five functioning scales of the QLQ-C30 varied from $35 \%$ for physical functioning to $55 \%$ for social and cognitive functioning. Global QoL improved in $36 \%$ of the cases. In this study, a significant association was found between objective tumour response and palliation of chest pain, pain arm/shoulder, fatigue and physical functioning. A significant association was also observed between radiation-induced pulmonary changes assessed with chest radiograph and dyspnea and fatigue.

\section{Chapter 8}

Chapter 8 describes the changes in QoL after curative radiotherapy. The response rates for the pulmonary and general symptoms were comparable with those observed after palliative and radical radiotherapy. A significant gradual increase with time was observed for dyspnea, chest pain and fatigue. A significant gradual deterioration was also observed for global QoL. No significant changes were observed for either the other symptoms or the functioning scales. Significantly higher levels of dysphagia were observed in those in which the regional lymph nodes were treated as well compared to the locally treated patients, which persisted up to 12 months. None of the patients developed a regional recurrence. Radiation-induced pulmonary changes assessed with chest radiograph were more pronounced in the group treated with loco-regional radiotherapy. Taking into account the low incidence of regional 
recurrences after local irradiation, the higher incidence and severity of radiation-induced changes and the higher levels of dysphagia persisting up to 12 months, local irradiation could be considered in small peripherally located tumours.

\section{Chapter 9}

In chapter 9 , the results of a retrospective study of 938 patients were presented. This study was conducted to investigate whether EBB was really a risk factor for massive haemoptysis in patients primarily treated by a combination of EBB and XRT for NSCLC. The incidence of massive haemoptysis was $10.8 \%$. A significantly higher incidence of massive haemoptysis was observed in those patients who were eligible for EBB but actually treated with XRT alone compared to those who were not eligible for EBB. Another important finding was that the incidence of massive haemoptysis depended significantly on the fraction size of brachytherapy. When 2 fraction of 7.5 Gy were used in combination with XRT, the incidence of massive haemoptysis was similar to that observed in those eligible for EBB but actually treated with XRT alone. However, when a single dose of $15 \mathrm{~Gy}$ was used, the incidence of massive haemoptysis increased dramatically. In a multivariate analysis, a single dose of 15 Gy EBB, haemoptysis before radiotherapy, tumour in the main bronchus and localisation of the tumour in one of the upper lobes were significantly associated with a higher incidence of massive haemoptysis. Based on the results of this study, two fractions of $7.5 \mathrm{~Gy}$ (at $1 \mathrm{~cm}$ ) with EBB in combination with XRT was considered feasible.

\section{Chapter 10}

In chapter 10, the results of a prospective randomised trial comparing XRT alone with XRT in combination with EBB were presented. The aim of this prospective randomized study was to test the hypothesis that the addition of EBB to XRT provided better palliation of dyspnea and other post-obstructive features such as post-obstructive pneumonia/atelectasis and finally quality of life, in patients with NSCLC with endobronchial tumor. Unfortunately, the study was ended prematurely due to insufficient patient accrual. Nevertheless, patients treated with concurrent EBB experienced a significantly higher rate of re-expansion of collapsed lung compared to those who received XRT alone. Subsequently, the level of palliation of dyspnea was significantly higher after XRT plus EBB compared to after XRT alone. However, this 
beneficial effect regarding relief of dyspnea was only observed up to 3 months after radiotherapy.

\section{Chapter 11}

Chapter 11 contains the general discussion and conclusions of this thesis. Given the relatively poor prognosis of patients with inoperable NSCLC and the limited gain expected from new modalities, QoL should be an endpoint in clinical studies investigating these new modalities. Although the response rates of pulmonary symptoms achieved with radiotherapy are satisfactory, the response rates for general symptoms and other aspects of QoL are lower than was expected. These findings may provide important information for future clinical trials. Objective tumour response and radiation-induced pulmonary changes may have some effect on changes of pulmonary symptoms but have limited effect on functioning and global QoL. EBB can be administered safely in combination with XRT, provided that the fraction dose of EBB is not higher that $10 \mathrm{~Gy}$ per fraction. In a randomised study, the addition of EBB to XRT appears to be beneficial regarding re-expansion of collapsed lung and relief of dyspnea. However, the premature ending of this study precludes definitive conclusions. 


\section{Samenvatting}




\section{a Hoofdstuk 1}

Hoofdstuk 1 bevat een algemene inleiding waarin verschillende aspecten van het meten van kwaliteit van leven en de evaluatie hiervan worden beschreven, zowel in het algemeen als voor longkanker patiënten in het bijzonder. Ondanks de relatief sombere prognose van patiënten met een inoperabel niet-kleincellig bronchuscarcinoom, (NSCLC), is de beschikbare informatie over kwaliteit van leven, gemeten met daarvoor gevalideerde en betrouwbare instrumenten, zeer beperkt.

\section{Hoofdstuk 2}

Hoofdstuk 2 beschrijft de doelstellingen van dit proefschrift en de opzet van de studie.

De doelstellingen van de studie waren het prospectief beschrijven van kwaliteit van leven voor en na radiotherapie bij patiënten met een inoperabel NSCLC, het bepalen van factoren die mogelijk van invloed zijn op veranderingen van kwaliteit van leven en het evalueren van de prognostische betekenis van kwaliteit van leven gemeten voor de behandeling. Hiervoor werd een prospectieve niet-gerandomiseerde studie gestart, waarin 264 patiënten werden geincludeerd. Op grond van een aantal helder gedefinieerde prognostische factoren werden deze patiënten verdeeld in een drietal prognostische groepen die vervolgens werden behandeld volgens drie verschillende fractioneringschema, i.e. het palliatieve, radicale en curatieve fractioneringschema. Bij al deze patiënten werd kwaliteit van leven gemeten voor, tijdens en 2 weken, 6 weken, 3, 6 en 12 maanden na radiotherapie, waarbij gebruik gemaakt werd van de EORTC QLQ-C30 en de longkanker module, de EORTC QLQ-LC13.

Een andere doelstelling was het vaststellen van de veiligheid en de waarde van de toevoeging van endobronchiale brachytherapie (EBB) aan uitwendige radiotherapie. Voor de bepaling van het meest optimale fractioneringsschema voor de EBB werd een retrospectieve studie verricht onder 938 patiënten met een inoperabel NSCLC. Voor de bepaling van de toevoegende waarde van EBB werd een prospectief gerandomiseerde studie geïnitieerd waarin patiënten met een centraal gelokaliseerde tumor werden gerandomiseerd tussen uitwendige radiotherapie alleen en uitwendige radiotherapie in combinatie met EBB. 


\section{- Hoofdstuk 3}

In dit hoofdstuk worden de kwaliteit van leven karakteristieken beschreven van de patiënten in de drie prognostische groepen. In de groep met de kortste levensverwachting werden significant hogere waarden gevonden voor pulmonale en algemene symptomen in vergelijking met de groepen met een betere levensverwachtingen. Tevens scoorden de patiënten geselecteerd voor het palliatieve fractioneringschema slechter met betrekking tot de functioneren en de giobale kwaliteit van leven. In de tweede plaats werd gekeken naar de relatie tussen kwaliteit van leven en de belangrijkste prognostische factoren. $A$ significante relatie werd gevonden tussen kwaliteit van leven en WHO performance status. Echter, de 'effect sizes' waren betrekkelijk laag, hetgeen betekent dat slechts een klein gedeelte van de variantie die werd waargenomen in de gemiddelde waarden van de kwaliteit van leven schalen kon worden verklaard uit variantie in de performance status. Tevens werd een relatie gevonden tussen gewichtsverlies en 'physical functioning' en 'role functioning' en een aantal algemene symptomen zoals moeheid en eetlustvermindering. Tegen de verwachting in werd geen relatie gevonden met tumor stadium. Tenslotte werd gekeken naar de relatie tussen pulmonale symptomen en algemene symptomen en kwaliteit van leven. Alleen dyspneu bleek significant gerelateerd aan de functionele schalen en de globale kwaliteit van leven.

\section{ㅁ Hoofdstuk 4}

In hoofdstuk 4 worden de resultaten beschreven van een prospectieve studie naar de prognostische betekenis van 'baseline' kwaliteit van leven in de groep patiënten met een inoperabel NSCLC die werden behandeld volgens een radicaal fractioneringschema. In de multivariate analyse, waarbij alleen de traditionele prognostische factoren werden meegenomen, bleken de WHO performance status (WHO 0 versus $W H O>0$ ), het gewichtsverlies $(0-10 \%$ versus $>10 \%)$ en de $\mathrm{N}$-status ( $\mathrm{N} 0$ versus $\mathrm{N}+$ ) onafhankelijke prognostische factoren te zijn voor de overleving. Indien de kwaliteit van leven parameters aan het multivariate model werden toegevoegd, bleek de globale kwaliteit van leven schaal de sterkste prognostische betekenis te hebben ten koste van de WHO performance status. Dit effect werd echter alleen gevonden in de groep patiënten met lymfkliermetastasen en niet in de klinische NO patiënten. Door een aantal prognostische factoren te combineren kon een kleine groep patiënten met een zeer korte 
overlevingsduur worden geïdentificeerd. Gezien deze zeer korte overlevingsduur lijkt radiotherapie volgens een palliatief schema in deze geselecteerde groep een betere optie.

\section{[ Hoofdstuk 5}

In hoofdstuk 5 worden de resultaten getoond van een prospectieve studie die werd verricht ter bepaling van de waarde van de $\mathrm{X}$-thorax bij het bepalen van tumor respons. Uit deze studie bleek dat CT-thorax meer geschikt was voor het meten van de grootte van de tumor dan de X-thorax. De X-thorax bleek echter even betrouwbaar voor het meten van de grootte van de tumor dan de CT-thorax bij tumoren die goed zichtbaar en afgrensbaar waren op de $\mathrm{X}$-thorax en daardoor goed te meten. In die gevallen werden geen belangrijke verschillen gevonden tussen $\mathrm{X}$-thorax en $\mathrm{CT}$-thorax met betrekking tot de respons classificatie en de verschillen in overleving tussen de responders en de nietresponders. Op grond van deze studie werd besloten alleen een CT-thorax te verrichten ter bepaling van de tumor respons in die gevallen waarin de tumor op de $\mathrm{X}$-thorax niet goed meetbaar was.

\section{Hoofdstuk 6}

In hoofdstuk 6 werden de veranderingen in kwaliteit van leven beschreven in de groep patiënten die werd behandeld volgens het palliatieve fractioneringschema. Het respons percentage voor hemoptoë was $79 \%$, voor pijn in de arm/schouder $56 \%$, voor pijn op de borst $53 \%$, voor hoest $49 \%$ en voor dyspneu $39 \%$. De respons percentages voor moeheid en gebrek aan eetlust waren laag. De respons percentages voor de vijf functionele schalen varieerde van $35 \%$ voor 'role functioning' tot $57 \%$ voor 'emotional functioning'. De globale kwaliteit van leven verbeterde in $37 \%$ van de gevallen. In het algemeen was er een tendens naar een betere palliatie van symptomen en verbetering van kwaliteit van leven in patiënten met een objectieve tumor respons in vergelijking met die patiënten waarin geen objectieve tumorrespons werd waargenomen.

\section{口 Hoofdstuk 7}

In dit hoofdstuk worden de veranderingen van kwaliteit van leven beschreven die werden waargenomen na radicale radiotherapie. De respons percentages waren uitstekend voor hemoptoë (83\%), goed voor pijn op de borst $(68 \%)$, pijn in de arm/schouder $(63 \%)$ en voor 
gebrek aan eetlust (59\%) maar teleurstellend voor dyspneu (37\%), hoest $(31 \%)$ en moeheid $(28 \%)$. De respons percentages voor de functionele schalen varieerde van $35 \%$ voor 'physical functioning' tot '55\% voor 'social functioning' en 'cognitive functioning'. Voor de globale kwaliteit van leven werd een respons percentage waargenomen van $35 \%$. In deze studie werd een verband gevonden tussen objectieve tumor respons en palliatie van pijn op de borst, arm/schouder pijn, moeheid en 'physical functioning'. Tevens werd een verband aangetoond tussen door radiotherapie geïnduceerde radiologische veranderingen en dyspneu.

\section{Hoofdstuk 8}

Hoofdstuk 8 beschrijft de veranderingen in kwaliteit van leven na curatieve radiotherapie. De respons percentages voor de pulmonale en algemene klachten waren vergelijkbaar met de percentages waargenomen na palliatieve en radicale radiotherapie. $\mathrm{Na}$ radiotherapie werd een langzame toename waargenomen van dyspneu, pijn op de borst en moeheid. Tevens werd een langzame verslechtering gezien voor de globale kwaliteit van leven. In de groep patiënten waarbij de mediastinale en ipsilaterale lymfklieren in het doelvolume werden opgenomen, werden significant meer klachten van dysfagie waargenomen dan na alleen lokale radiotherapie. Dit verschil in dysfagie was nog na 12 maanden nog steeds aanwezig. Geen van de patiënten ontwikkelde lymfogene metastasen. Radiatie-geinduceerde radiologische veranderingen op de $\mathrm{X}$-thorax waren meer uitgesproken aanwezig na loco-regionale radiotherapie dan na lokale radiotherapie. Gezien de lage incidentie van lymfogene metastasen na lokale radiotherapie bij stadium I NSCLC en de verschillen ten aanzien van radiatie geïnduceerde schade aan de long en de dysfagie ten nadele van loco-regionale radiotherapie, zou lokale radiotherapie bij kleine perifeer gelokaliseerde tumoren de voorkeur moeten krijgen.

\section{ㄱoofdstuk 9}

In hoofdstuk 9 worden de resultaten van een retrospectieve studie getoond naar de risicofactoren voor een massale longbloeding bij patiënten met een inoperabel NSCLC die werden behandeld met uitwendige radiotherapie, EBB of een combinatie van deze twee modaliteiten. The incidentie van massale longbloedingen was $10.8 \%$ voor de gehele groep. Een significant hogere incidentie aan massale longbloedingen werd waargenomen 
bij die patiënten die geschikt waren voor EBB maar alleen waren behandeld met uitwendige radiotherapie in vergelijking met patiënten die niet geschikt waren voor EBB. Een belangrijke bevinding was tevens dat het fractioneringschema van de brachytherapie van belang was. Indien 2 fracties van 7.5 Gy (gedoseerd op 1 centimeter van de bronas) werden gegeven in combinatie met uitwendige radiotherapie was de incidentie van massale longbloedingen gelijk aan de incidentie die werd waargenomen in de groep patiënten die met in principe geschikt waren voor EBB maar alleen met uitwendige radiotherapie waren behandeld. Na een eenmalige dosis van 15 Gy (gedoseerd op 1 centimeter van de bronas) in combinatie met uitwendige radiotherapie, werd een dramatische toename gezien van de incidentie van massale longbloedingen. In een multivariate analyse bleken de volgende factoren een verhoogd risico te geven op het overlijden aan een massale longbloeding, namelijk een eenmalige dosis van 15 Gy EBB, hemoptoë voor de behandeling, een tumor in de hoofdbronchus en een tumor gelokaliseerd in een van de bovenkwabsbronchi. Geconcludeerd werd dat EBB kan worden toegepast in combinatie met uitwendige radiotherapie, mits de EBB op een juiste wijze wordt gefractioneerd, b.v. 2 × 7.5 Gy op 1 centimeter van de bronas.

\section{Hoofdstuk 10}

Hoofdstuk 10 beschrijft de resultaten van en prospectief gerandomiseerde studie waarin uitwendige radiotherapie alleen werd vergeleken met de combinatie van gelijktijdige EBB ( $2 \times 7.5$ Gy op dag 1 en 8) en uitwendige radiotherapie. Het doel van de studie was het testen van de hypothese dat de toevoeging van EBB aan uitwendige radiotherapie zou leiden tot een betere palliatie van dyspneu en andere post-obstructieve verschijnselen zoals obstructieve pneumonie en/of atelectase en uiteindelijk ook ten aanzien van de kwaliteit van leven. Helaas werd de studie voortijdig gesloten in verband met een te lage instroom. In totaal werden 95 van de benodigde 160 patiënten geincludeerd. Desalniettemin bleken de patiënten die werden behandeld met de combinatie van uitwendige radiotherapie en EBB een significant hogere respons te vertonen voor het opheffen van atelectase in vergelijking met de patiënten die alleen uitwendig werden bestraald. Tevens werd een betere palliatie van dyspneu gezien in de gecombineerd behandelde groep, met dien verstande dat dit verschil na 3 maanden verdween en uitsluitend werd gezien in de groep met een obstruerende tumor in een van de 
hoofdbronchi. Dit effect werd echter niet waargenomen bij patiënten met een tumor in de lobaire bronchi.

\section{Hoofdstuk 11}

Hoofdstuk 11 bevat de algemene beschouwing en de conclusies van het onderzoek. Gezien de korte levenverwachting van patienten met een inoperabel NSCLC en de beperkte te verachten winst van nieuwe modaliteiten, zou kwaliteit van leven een belangrijk eindpunt moeten zijn in klinische studies waarin deze patienten worden geincludeerd. De studies tonen aan dat ook in een populatie patienten met een relatief korte levensverwachting een hoge compliance ten aanzien van het meten van kwaliteit te bereiken is.

Hoewel de respons percentages voor de pulmonale symptomen bevredigend zijn, vallen de resultaten ten aanzien van de palliatie van algemene klachten en ten aanzien van verbetering van kwaliteit van leven enigzins tegen.

De resultaten tonen dat objectieve tumor respons die wordt bereikt met radiotherapie een mechanisme is waarmee palliatie van sommige pulmonale klachten wordt bereikt. Objectieve tumorrespons kan echter niet worden gebruikt ais surrogaat voor palliatie.

De toevoeging van endobronchiale brachytherapie aan uitwendige radiotherapie bij patienten met een endobronchiaal gelokaliseerde tumor leidt niet tot een toename van het risico op massale longbloedingen, mits de fractiedosis die voor de brachytherapie wordt gebruikt niet te hoog gekozen wordt. De toevoegende waarde van endobronchiale brachytherapie lijkt echter beperkt. Alleen bij patienten met een obstruerende tumor in een van de hoofdbronchi werd een betere palliatie bereikt van dyspneu, die echter van beperkte duur was. 



\section{Dankwoord}

Zoals een ieder die zelf ooit een boekje als dit heeft geschreven weet, komt een proefschrift slechts tot stand met de onmisbare hulp van velen.

Allereerst zou ik mijn promotor Miel Wouters, willen bedanken.

Beste Miel, op de 'dinsdagen tussen de middag' heb ik niet alleen boterhammen, maar ook heel wat uren van jouw tijd opgepeuzeld. Jouw inzicht in de methodologische aspecten bij het verrichten van klinische studies en jouw gave overzicht over het geheel te behouden, hebben mij wetenschappelijk op het rechte pad gehouden.

Tevens dank aan mijn beide copromotoren, Jos de Jong en Guul ten Velde.

Beste Jos, het grootste deel van de tijd dat dit onderzoek werd verricht, was jij niet alleen mijn opleider, maar ook degene die mij de mogelijkheid heeft geboden dit proefschrift te schrijven. Jouw inspanningen om de juiste balans te vinden tussen opleiding en onderzoek verdient lof en zou een voorbeeld voor velen moeten zijn. Van jouw oog voor detail krijgt een ieder die door jouw is opgeleid iets mee en ik heb er nog steeds plezier van.

Beste Guul, zelden heb ik iemand meegemaakt die de door mij aangeleverde manuscripten zo snel en grondig doornam en van commentaar voorzag als jij. Jouw kritische commentaar, vaak begeleid door de woorden 'als longarts zou ik ook nog willen weten' hebben mij niet alleen vaak aan het denken en het werk gezet maar ook enig inzicht gegeven in datgene wat er in een longartsenbrein omgaat.

Een bijzonder woord van dank zou ik ook willen wijden aan twee experts op het gebied van kwaliteit van leven, Neil Aaronson en Martin Muller.

Beste Neil, jouw kennis over kwaliteit van leven en de enorme inspanningen die jij hebt verricht bij het zowel inhoudelijk als tekstueel beoordelen van de manuscripten zijn van onschatbare waarde geweest bij het tot stand komen van dit proefschrift.

Beste Martin, jouw inspanningen en kundige adviezen op het gebied van de analyse van kwaliteit van leven onderzoek waren niet alleen statistisch maar ook voor dit proefschrift significant.

Annet de Mol was verantwoordelijk voor het verzamelen van de vragenlijsten. 
Beste Annet, dankzij jouw inspanningen en niet aflatende ijver waren deze kwaliteit van leven studies niet tot een goed einde gekomen. Ook na mijn vertrek uit Heerlen hield jij trouw een oogje in het zeil en bleven de vragenlijsten binnenstromen.

Rob Lamers was mede verantwoordelijk voor het beoordelen en herbeoordelen van alle radiologische informatie. Beste Rob, we hebben heel wat uren voor de lichtkast doorgebracht. Zonder jouw bijdrage zouden vele vragen onbeantwoord zijn gebleven. Bovendien heb ik een hoop kennis en vaardigheden opgedaan bij de beoordeling van de honderden foto's and scans.

Martin Tjwa verrichtte het overgrote merendeel van de bronchoscopieën voor de endobronchiale brachytherapie. Beste Martin, jouw hulp bij de brachytherapie was onontbeerlijk. Hartelijk dank voor de plezierige samenwerking.

De resultaten beschreven in dit proefschrift zouden nooit tot stand zijn gekomen zonder de hulp van Janet Beukema, Wim Dubois, Sherif El Sharouni, Piet van den Ende, Danielle de Haas, Niek van Hasselt, Jos Jager, Ludy Lutgens, Jacqueline Peer, llona Schmeets, Tom Verschueren, Antoinette van der Wel, Peter-Paul van der Toorn, Jeroen Uppelschoten en Rinus Wanders, die gedurende vele jaren honderden patienten includeerden.

Tot slot wil ik Tineke, Marieke, Carolien en Lisa noemen. Lieve Tineke, zonder jouw geduld en begrip had ik het nooit gered. Je bent een fantastische vrouw. Lieve Marieke, Carolien en Lisa, dit is nu het proefschrift. Ik kan jullie nog steeds niet uitleggen wat dat nu precies is. Jullie zijn onmisbaar voor mijn kwaliteit van leven. Daar hoef ik in ieder geval geen onderzoek naar te doen. 


\section{Curriculum vitae}

1963 Geboren te Amsterdam

1975-1982 Gymnasium B, CSG Sweelinck in Amsterdam

1982-1989 Studie Geneeskunde aan de Vrije Universiteit in Amsterdam

1989-1990 Verzekeringsgeneeskundige bij de Gemeenschappelijke Medische Dienst in Apeldoorn

1990-1992 Arts-assistent (AGNIO) interne geneeskunde in het Andreas Ziekenhuis in Amsterdam

1992-1993 Arts-assistent (AGNIO) radiotherapie in het Radiotherapeutisch Instituut Limburg in Heerlen

1993-1997 Arts-assistent in opleiding tot radiotherapeut in het Radiotherapeutisch Instituut Limburg in Heerlen (opleider dr. J.M.A. de Jong)

Vanaf 1997 Werkzaam als radiotherapeut op de Afdeling Radiotherapie van het Academisch Ziekenhuis Vrije Universiteit in Amsterdam 